\title{
C-O Bond Silylation Catalyzed by Iron: A General Method for the Construction of $\mathrm{Csp}^{2}-\mathrm{Si}$ Bonds
}

Juan Zhang, ${ }^{1}$ Yun Zhang, ${ }^{1}$ Shasha Geng, ${ }^{1}$ Shuo Chen, ${ }^{1}$ Zhengli Liu, ${ }^{1}$ Xiaoqin Zeng, ${ }^{1}$ Yun $\mathrm{He},{ }^{1}$ and Zhang Feng ${ }^{1,2 *}$

${ }^{1}$ Chongqing Key Laboratory of Natural Product Synthesis and Drug Research, Chemical Biology Research Center, School of Pharmaceutical Sciences, Chongqing University, Chongqing 401331, P. R. China

${ }^{2}$ Sichuan Key Laboratory of Medical Imaging \& Department of Chemistry, School of Preclinical Medicine, North Sichuan Medical College, Nanchong, Sichuan 637000, China

\section{List of Contents}

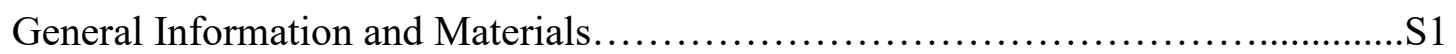

Optimization of Iron-Catalyzed Silylation of Aryl Carbamates.....................S2

Optimization of Iron-Catalyzed Silylation of Alkenyl Carbamates....................S5

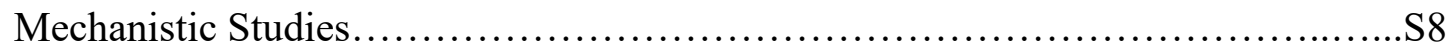

General Procedure for the Synthesis of Carbamates............................S1 1

General Procedure for the Silylation of Carbamates............................S13

Experimental Procedures and Characterization Data for Products.................S16

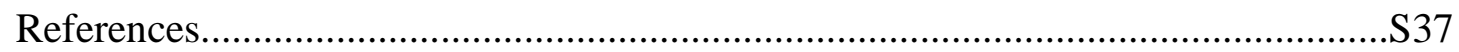

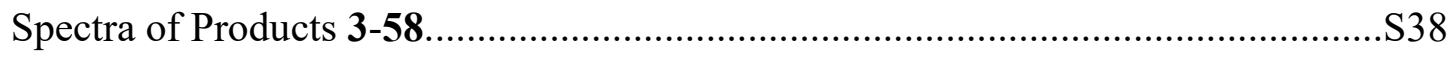


General information: ${ }^{1} \mathrm{H}$ NMR and ${ }^{13} \mathrm{C}$ NMR spectra were recorded on Agilent 400MR DD2 (400 MHz) or 600MR DD2 (600 MHz) spectrometer at ambient temperature. Chemical shifts $(\delta)$ are reported in ppm, and coupling constants $(J)$ are in Hertz $(\mathrm{Hz})$. The following abbreviations were used to explain the multiplicities: $\mathrm{s}=$ singlet, $\mathrm{d}=$ doublet, $\mathrm{t}=$ triplet, $\mathrm{q}=$ quartet, $\mathrm{m}=$ multiplet, sept $=$ septet. $\mathrm{NMR}$ yield was determined by ${ }^{1} \mathrm{H}$ NMR using mesitylene as an internal standard before working up the reaction.

Materials: All reagents that used were from commercial sources, unless otherwise specified. $\mathrm{Fe}(\mathrm{OTf})_{2}(97 \%)$ was purchased from Adamas. $\mathrm{Fe}(\mathrm{OAc})_{2}(>90 \%)$ was purchased from TCI. FeI (97\%) was purchased from Alfa. $t$-BuONa (99\%), MeOLi (98\%) and $\mathrm{MeONa}(98 \%)$ were purchased from Adamas. Diglyme and DMF were distilled under reduced pressure from $\mathrm{CaH}_{2}$. 1, 4-Dioxane, THF, MTBE, $(i \text {-Pr })_{2} \mathrm{O}$ and toluene were distilled from sodium and benzophenone immediately before used. 
Optimization of Iron-Catalyzed Silylation of Aryl Carbamates (Table S1-S6):

Table S1. Screening of Ligands. ${ }^{a}$<smiles>CCN(CC)C(=O)Oc1cccc2ccccc12</smiles>

$1 \mathrm{a}$

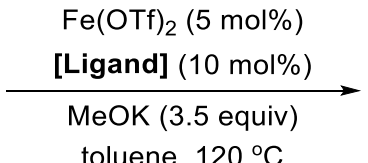

toluene, $120^{\circ} \mathrm{C}$

2a (2.5 equiv)

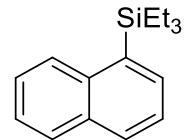

3

\begin{tabular}{ccc}
\hline Entry & Ligand & Yield $^{b}$ \\
\hline 1 & TMEDA & $0 \%$ \\
2 & Phenanthroline & $0 \%$ \\
3 & Biquinoline & $0 \%$ \\
4 & Bipyridine & $0 \%$ \\
5 & DPPM & $0 \%$ \\
6 & DPPP & $0 \%$ \\
7 & PCy & $0 \%$ \\
8 & $\mathrm{P}(t-\mathrm{Bu})_{3}$ & $0 \%$ \\
9 & $\mathrm{P}(p \text {-tol })_{3}$ & $28 \%$ \\
$\mathbf{1 0}$ & $\mathbf{X P h o s}$ & $\mathbf{3 6 \%}$ \\
11 & none & $0 \%$ \\
\hline
\end{tabular}

${ }^{a}$ Reaction conditions (unless otherwise specified): 1a ( 0.3 mmol, 1.0 equiv), $\mathbf{2 a}$ (2.5 equiv), MeOK (3.5 equiv), $\mathrm{Fe}(\mathrm{OTf})_{2}(5 \mathrm{~mol} \%)$, ligand $(10 \mathrm{~mol} \%)$, toluene $(1.5 \mathrm{~mL}), 120^{\circ} \mathrm{C}, 15 \mathrm{~h}$, under an argon atmosphere. ${ }^{b}$ Determined by ${ }^{1} \mathrm{H}$ NMR using mesitylene as an internal standard.

Table S2. Screening of Bases. ${ }^{a}$

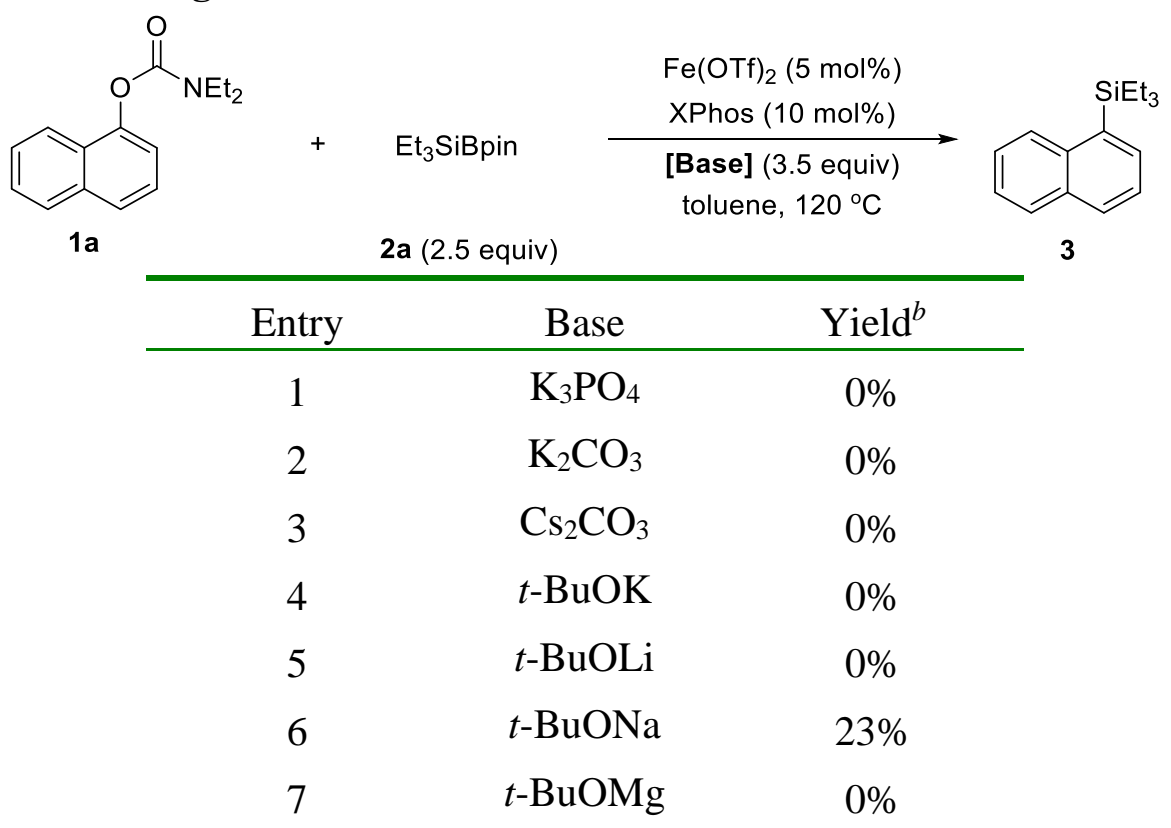




\begin{tabular}{ccc}
$\mathbf{8}$ & MeONa & $\mathbf{6 0 \%}$ \\
9 & $\mathrm{MeOK}$ & $36 \%$ \\
10 & $\mathrm{MeOLi}$ & $10 \%$ \\
11 & $\mathrm{PhONa}$ & $0 \%$ \\
12 & none & $0 \%$ \\
\hline
\end{tabular}

${ }^{a}$ Reaction conditions (unless otherwise specified): $\mathbf{1 a}(0.3 \mathrm{mmol}, 1.0$ equiv), $\mathbf{2 a}$ ( 2.5 equiv), base (3.5 equiv), $\mathrm{Fe}(\mathrm{OTf})_{2}(5 \mathrm{~mol} \%)$, XPhos $(10 \mathrm{~mol} \%)$, toluene $(1.5 \mathrm{~mL}), 120^{\circ} \mathrm{C}, 15 \mathrm{~h}$, under an argon atmosphere. ${ }^{b}$ Determined by ${ }^{1} \mathrm{H}$ NMR using mesitylene as an internal standard.

Table S3. Screening of Solvents. ${ }^{a}$<smiles>CCNC(=O)Oc1cccc2ccccc12</smiles>

1a $+\quad \mathrm{Et}_{3} \mathrm{SiBpin}$

2a (2.5 equiv)

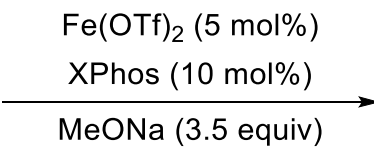

[Solvent], $120^{\circ} \mathrm{C}$<smiles>CCCc1cccc2ccccc12</smiles>

3

\begin{tabular}{ccc}
\hline Entry & Solvent & Yield $^{b}$ \\
\hline 1 & Diglyme & $6 \%$ \\
2 & $(i-\mathrm{Pr})_{2} \mathrm{O}$ & $28 \%$ \\
3 & $\mathrm{PhCF}_{3}$ & $18 \%$ \\
4 & Toluene & $60 \%$ \\
$\mathbf{5}$ & Dioxane & $\mathbf{6 4 \%}$ \\
6 & MTBE & $15 \%$ \\
7 & MeCN & $0 \%$ \\
8 & NMP & $0 \%$ \\
9 & DMSO & $0 \%$ \\
10 & DCM & $0 \%$ \\
\hline
\end{tabular}

${ }^{a}$ Reaction conditions (unless otherwise specified): $\mathbf{1 a}(0.3 \mathrm{mmol}, 1.0$ equiv), $\mathbf{2 a}$ ( 2.5 equiv), $\mathrm{MeONa}$ (3.5 equiv), $\mathrm{Fe}(\mathrm{OTf})_{2}(5 \mathrm{~mol} \%)$, XPhos $(10 \mathrm{~mol} \%)$, solvent $(1.5 \mathrm{~mL}), 120^{\circ} \mathrm{C}, 15 \mathrm{~h}$, under an argon atmosphere. ${ }^{b}$ Determined by ${ }^{1} \mathrm{H}$ NMR using mesitylene as an internal standard.

Table S4. Screening of Iron Sources. ${ }^{a}$ 
<smiles>C[N+]C(=O)Oc1cccc2ccccc12</smiles>

$1 \mathrm{a}$

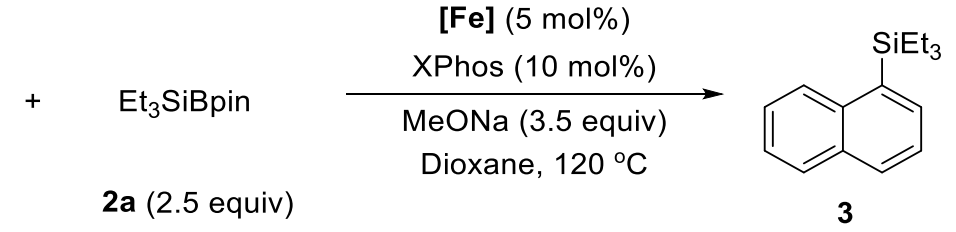

2a (2.5 equiv)

\begin{tabular}{ccc}
\hline Entry & {$[\mathrm{Fe}]$} & Yield $^{b}$ \\
\hline 1 & $\mathrm{Fe}(\mathrm{OTf})_{2}$ & $64 \%$ \\
2 & $\mathrm{Fe}(\mathrm{OTf})_{3}$ & $56 \%$ \\
3 & $\mathrm{Fe}(\mathrm{acac})_{2}$ & $43 \%$ \\
4 & $\mathrm{FeBr}_{2}$ & $78 \%$ \\
$\mathbf{5}$ & $\mathbf{F e}(\mathbf{O A c})_{2}$ & $\mathbf{9 3 \%}(\mathbf{8 9 \%})$ \\
6 & $\mathrm{FeCl}_{3}$ & $49 \%$ \\
7 & $\mathrm{Fe}\left(\mathrm{ClO}_{4}\right)_{2} \cdot \mathrm{xH}_{2} \mathrm{O}$ & $53 \%$ \\
8 & $\mathrm{~K}_{3} \mathrm{Fe}(\mathrm{CN})_{6}$ & $10 \%$ \\
9 & none & $0 \%$ \\
\hline
\end{tabular}

${ }^{a}$ Reaction conditions (unless otherwise specified): $\mathbf{1 a}(0.3 \mathrm{mmol}, 1.0$ equiv), $\mathbf{2 a}$ ( 2.5 equiv), $\mathrm{MeONa}$ (3.5 equiv), $[\mathrm{Fe}](5 \mathrm{~mol} \%)$, XPhos $(10 \mathrm{~mol} \%)$, dioxane $(1.5 \mathrm{~mL}), 120{ }^{\circ} \mathrm{C}, 15 \mathrm{~h}$, under an argon atmosphere. ${ }^{b}$ Determined by ${ }^{1} \mathrm{H}$ NMR using mesitylene as an internal standard. The isolated yield is shown in parentheses.

Table S5. Control Experiments of Silylation of Aryl Carbamates 1a. ${ }^{a}$<smiles>CCNC(=O)Oc1cccc2ccccc12</smiles>

$1 \mathrm{a}$

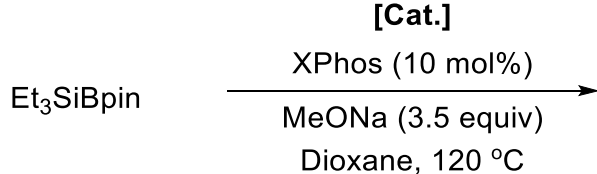

2a (2.5 equiv)<smiles>CCCc1cccc2ccccc12</smiles>

3

\begin{tabular}{cccc}
\hline Entry & Cat. & Loading & Yield $^{b}$ \\
\hline 1 & $\mathrm{Fe}(\mathrm{OAc})_{2}$ & $5 \%$ & $93 \%$ \\
2 & $99.99 \% \mathrm{Fe}(\mathrm{OAc})_{2}$ & $5 \%$ & $91 \%$ \\
3 & $\mathrm{Cu}(\mathrm{OAc})_{2}$ & $5 \%$ & $0 \%$ \\
4 & $\mathrm{CuI}$ & $5 \%$ & $0 \%$ \\
5 & $\mathrm{Pd}(\mathrm{OAc})_{2}$ & $0.1 \%$ & $0 \%$ \\
6 & $\mathrm{Pd}(\mathrm{OAc})_{2}$ & $1 \%$ & $0 \%$ \\
7 & $\mathrm{NiCl}_{2} \cdot 6 \mathrm{H}_{2} \mathrm{O}$ & $0.5 \%$ & $0 \%$ \\
8 & $\mathrm{NiCl}_{2} \cdot 6 \mathrm{H}_{2} \mathrm{O}$ & $5 \%$ & $0 \%$ \\
\hline
\end{tabular}


${ }^{a}$ Reaction conditions (unless otherwise specified): $\mathbf{1 a}(0.3 \mathrm{mmol}, 1.0$ equiv), $\mathbf{2 a}$ ( 2.5 equiv), $\mathrm{MeONa}$ (3.5 equiv), dioxane $(1.5 \mathrm{~mL}), 120{ }^{\circ} \mathrm{C}, 15 \mathrm{~h}$, under an argon atmosphere. ${ }^{b}$ Determined by ${ }^{1} \mathrm{H}$ NMR using mesitylene as an internal standard.

Table S6. Evaluation of Trace Metals Present in Commercial Iron Sources.

\begin{tabular}{|c|c|}
\hline \multicolumn{2}{|c|}{ Trace-metal analysis: $\mathrm{Fe}(\mathrm{OAc})_{2}(99.99 \%)$, Sigma-Aldrich } \\
\hline Element & Molar Concentration \\
\hline $\mathrm{Cu}$ & $<0.16 \mathrm{ppm}$ \\
\hline $\mathrm{Pd}$ & $<0.88 \mathrm{ppm}$ \\
\hline $\mathrm{Ni}$ & $<1.14 \mathrm{ppm}$ \\
\hline \multicolumn{2}{|c|}{ Trace-metal analysis: $\mathrm{FeI}_{2}(99.99 \%)$, Alfa Aesar } \\
\hline Element & Molar Concentration \\
\hline $\mathrm{Cu}$ & $<0.66 \mathrm{ppm}$ \\
\hline $\mathrm{Pd}$ & $<0.16 \mathrm{ppm}$ \\
\hline $\mathrm{Ni}$ & $<0.67 \mathrm{ppm}$ \\
\hline
\end{tabular}

Optimization of Iron-Catalyzed Silylation of Alkenyl Carbamates (Table S7S11):

Table S7. Screening of Iron Sources and Bases. ${ }^{a}$

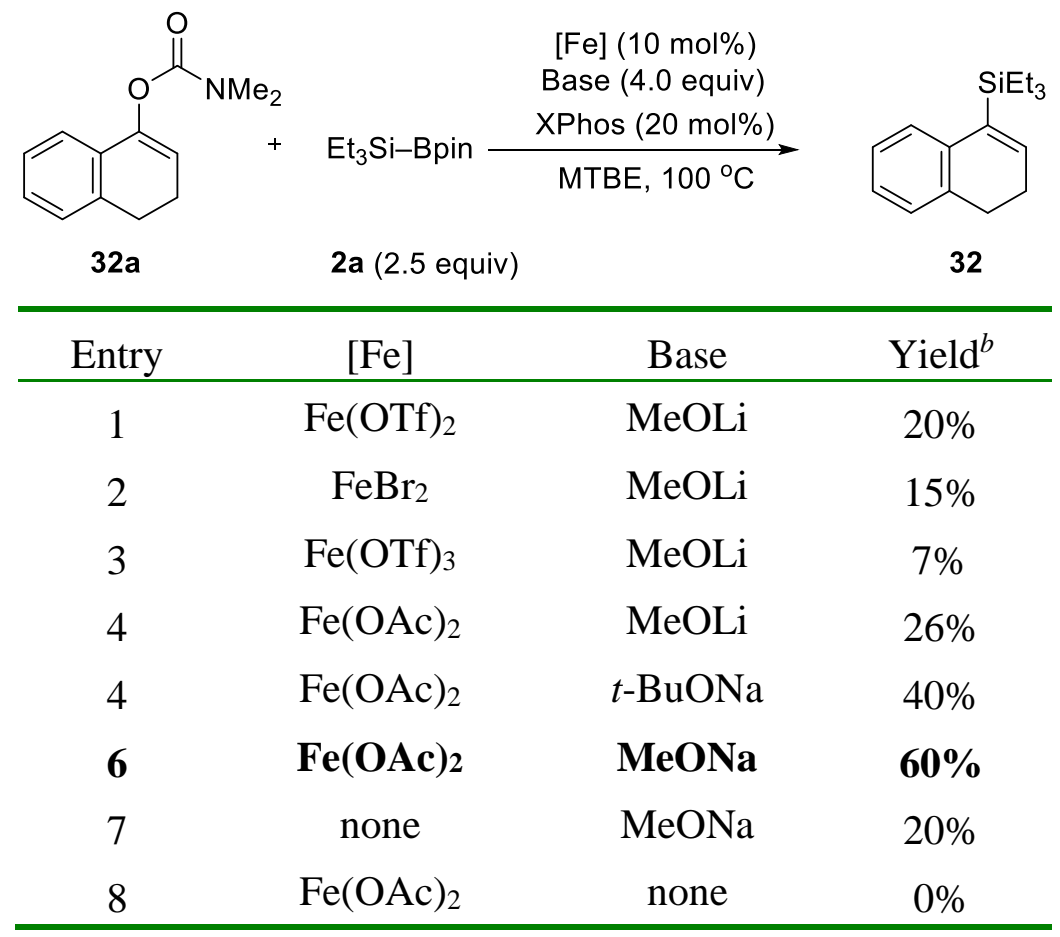


${ }^{a}$ Reaction conditions (unless otherwise specified): 32a ( $0.2 \mathrm{mmol}, 1.0$ equiv), $2 \mathbf{a}$ ( 2.5 equiv), base (4.0 equiv), MTBE $(2 \mathrm{~mL}), 100{ }^{\circ} \mathrm{C}, 15 \mathrm{~h}$, under an argon atmosphere. ${ }^{b}$ Determined by ${ }^{1} \mathrm{H}$ NMR using mesitylene as an internal standard.

Table S8. Screening of Ligands. ${ }^{a}$

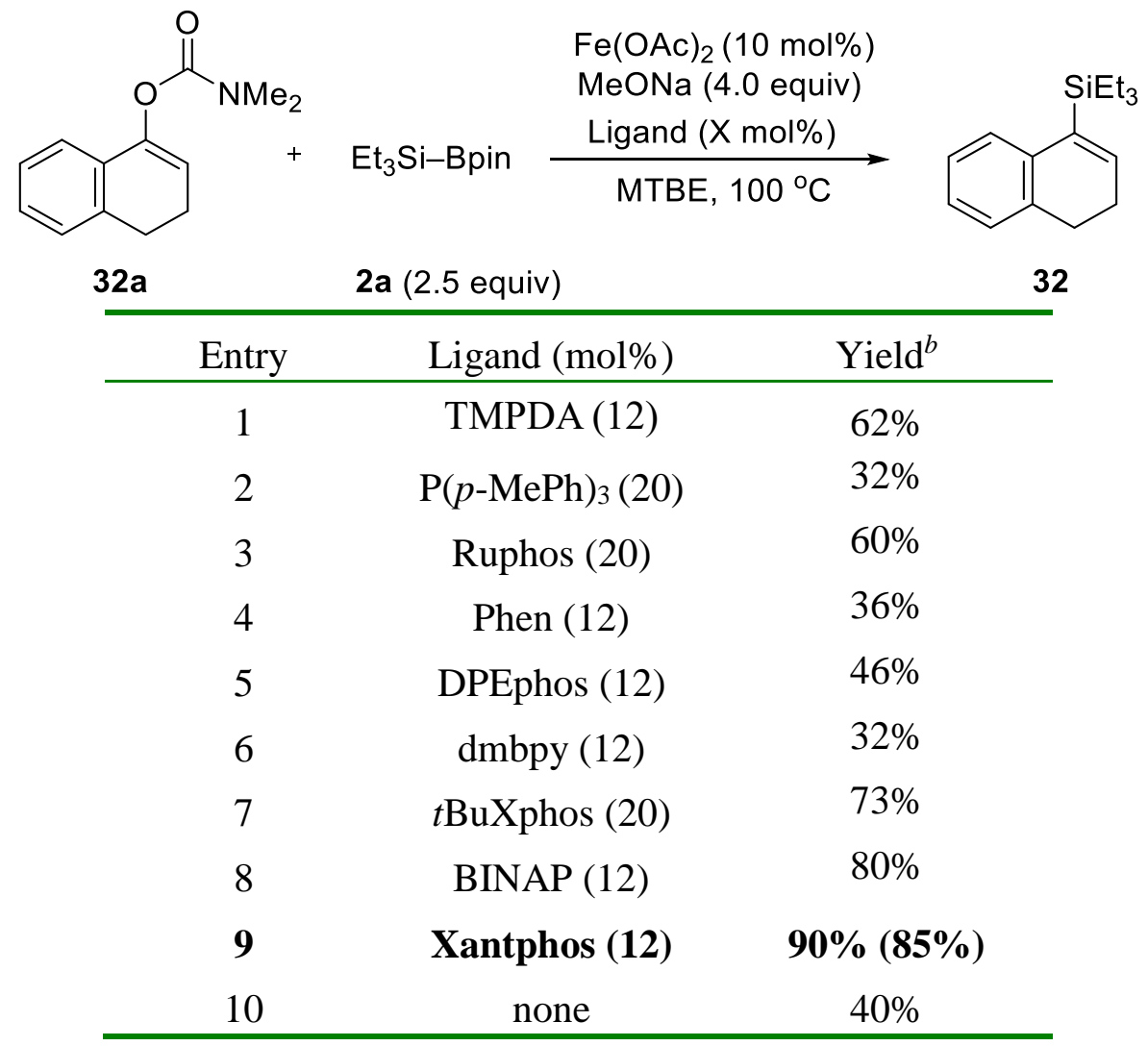

${ }^{a}$ Reaction conditions (unless otherwise specified): 32a ( $0.2 \mathrm{mmol}, 1.0$ equiv), 2a (2.5 equiv), $\mathrm{MeONa}$ (4.0 equiv), $\mathrm{Fe}(\mathrm{OAc})_{2}(10 \mathrm{~mol} \%)$, ligand, $\mathrm{MTBE}(2.0 \mathrm{~mL}), 100{ }^{\circ} \mathrm{C}, 15 \mathrm{~h}$, under an argon atmosphere. ${ }^{b}$ Determined by ${ }^{1} \mathrm{H}$ NMR using mesitylene as an internal standard. The isolated yield is shown in parentheses.

Table S9. Screening of Solvents and Temperature. ${ }^{a}$

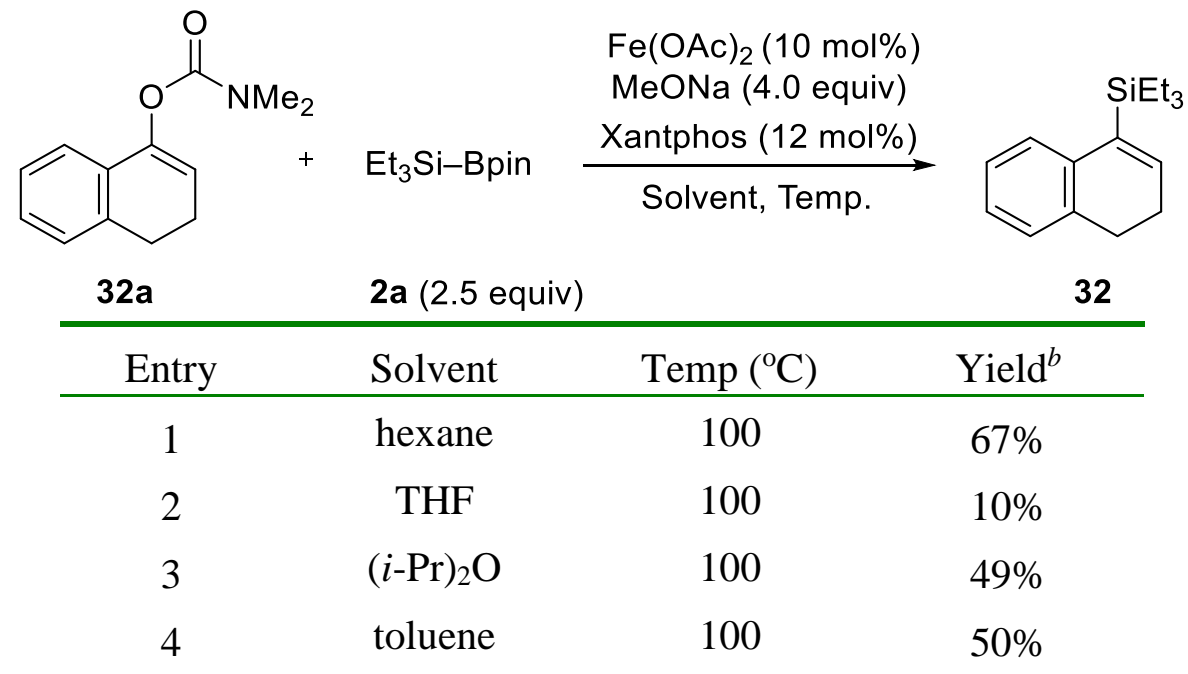




\begin{tabular}{cccc}
5 & dioxane & 100 & $43 \%$ \\
$\mathbf{6}$ & MTBE & $\mathbf{1 0 0}$ & $\mathbf{9 0 \%}$ \\
7 & MTBE & 90 & $77 \%$ \\
8 & MTBE & 120 & $83 \%$ \\
\hline
\end{tabular}

${ }^{a}$ Reaction conditions (unless otherwise specified): 32a $(0.2 \mathrm{mmol}, 1.0$ equiv), 2a (2.5 equiv), $\mathrm{MeONa}$ (4.0 equiv), $\mathrm{Fe}(\mathrm{OAc})_{2}(10 \mathrm{~mol} \%)$, Xantphos (12\%), solvent $(2.0 \mathrm{~mL}), 15 \mathrm{~h}$, under an argon atmosphere. ${ }^{b}$ Determined by ${ }^{1} \mathrm{H}$ NMR using mesitylene as an internal standard.

Table S10. Control Experiments of Iron-Catalyzed Silylation of 32a. ${ }^{a}$<smiles>CNC(=O)OC1=CCCc2ccccc21</smiles>

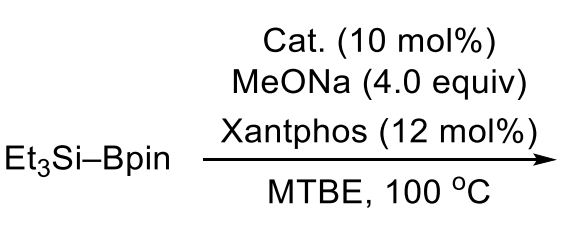

2a (2.5 equiv)<smiles>CCCC1=CCCc2ccccc21</smiles>

32

\begin{tabular}{ccccc}
\hline Entry & [Cat.] & Base & Ligand & Yield $^{b}$ \\
\hline 1 & $99.99 \% \mathrm{Fe}(\mathrm{OAc})_{2}$ & $\mathrm{MeONa}$ & Xantphos & $70 \%$ \\
2 & $\mathrm{FeI}$ & $\mathrm{MeONa}$ & Xantphos & $78 \%$ \\
3 & $\mathrm{Cu}(\mathrm{OAc})_{2}$ & $\mathrm{MeONa}$ & Xantphos & $45 \%$ \\
4 & $\mathrm{Ni}(\mathrm{OAc})_{2}$ & $\mathrm{MeONa}$ & Xantphos & $0 \%$ \\
5 & $\mathrm{Ni}(\mathrm{OTf})_{2}$ & $\mathrm{MeONa}$ & Xantphos & $0 \%$ \\
\hline
\end{tabular}

${ }^{a}$ Reaction conditions (unless otherwise specified): 32a $(0.2 \mathrm{mmol}, 1.0$ equiv), 2a (2.5 equiv), MeONa (4.0 equiv), catalysts (10 mol\%), Xantphos (12 mol\%), MTBE $(2.0 \mathrm{~mL}), 100{ }^{\circ} \mathrm{C}, 15 \mathrm{~h}$, under an argon atmosphere. ${ }^{b}$ Determined by ${ }^{1} \mathrm{H}$ NMR using mesitylene as an internal standard.

Table S11. Optimization of Iron-Catalyzed Silylation of 49a.

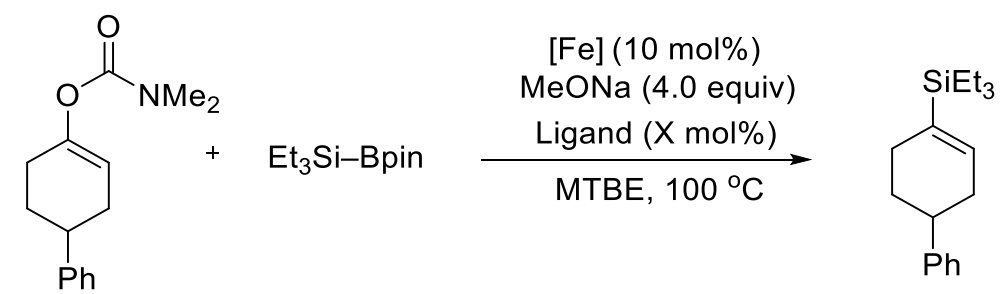

$49 a$

2a (3.0 equiv)

49

\begin{tabular}{ccccc}
\hline Entry & {$[\mathrm{Fe}]$} & Base & Ligand $(\mathrm{mol} \%)$ & Yield $^{b}$ \\
\hline 1 & $\mathrm{Fe}(\mathrm{OTf})_{2}$ & $\mathrm{MeONa}$ & Davephos $(20)$ & $20 \%$ \\
2 & $\mathrm{Fe}(\mathrm{OTf})_{2}$ & $\mathrm{MeONa}$ & XPhos $(20)$ & $48 \%$ \\
3 & $\mathrm{Fe}(\mathrm{OTf})_{2}$ & $\mathrm{MeONa}$ & TMEDA $(12)$ & $26 \%$ \\
4 & $\mathrm{Fe}(\mathrm{OTf})_{2}$ & $\mathrm{MeONa}$ & BINAP $(12)$ & $40 \%$ \\
5 & $\mathrm{Fe}(\mathrm{OAc})_{2}$ & $\mathrm{MeONa}$ & BINAP $(12)$ & $19 \%$
\end{tabular}




$\begin{array}{ccccc}6 & \mathrm{FeI}_{2} & \mathrm{MeONa} & \mathrm{BINAP}(\mathbf{1 2}) & \mathbf{6 5 \%}(\mathbf{6 0 \%}) \\ 7 & \mathrm{FeI}_{2} & \mathrm{MeONa} & \mathrm{L}_{1}(12) & 28 \% \\ 8 & \mathrm{FeI}_{2} & \mathrm{MeONa} & \mathrm{L}_{2}(12) & 35 \% \\ 9 & \mathrm{FeI}_{2} & \mathrm{MeONa} & \mathrm{L}_{3}(12) & 55 \% \\ 10 & \mathrm{FeI}_{2} & \mathrm{MeONa} & \mathrm{L} 4(12) & 66 \% \\ 11 & \mathrm{MeONa} & \mathrm{BINAP}(12) & 0 \%\end{array}$

${ }^{a}$ Reaction conditions (unless otherwise specified): 49a ( $0.2 \mathrm{mmol}, 1.0$ equiv), $2 \mathrm{a}$ ( 2.5 equiv), $\mathrm{MeONa}\left(4.0\right.$ equiv), $[\mathrm{Fe}](10 \mathrm{~mol} \%)$, ligand, MTBE $(2.0 \mathrm{~mL}), 100{ }^{\circ} \mathrm{C}, 15 \mathrm{~h}$, under an argon atmosphere. ${ }^{b}$ Determined by ${ }^{1} \mathrm{H}$ NMR using mesitylene as an internal standard. The isolated yield is shown in parentheses.

\section{Mechanistic Studies}

\section{Radical Inhibition Experiments (Table S12-S13).}

To gain some insights into the mechanism of this iron catalyzed $\mathrm{C}-\mathrm{O}$ bond activation reactions, radical inhibition experiments were conducted (Table S12-S13).

Table S12. Radical Inhibition Experiments of Iron-Catalyzed Silylation of 1a. ${ }^{a}$

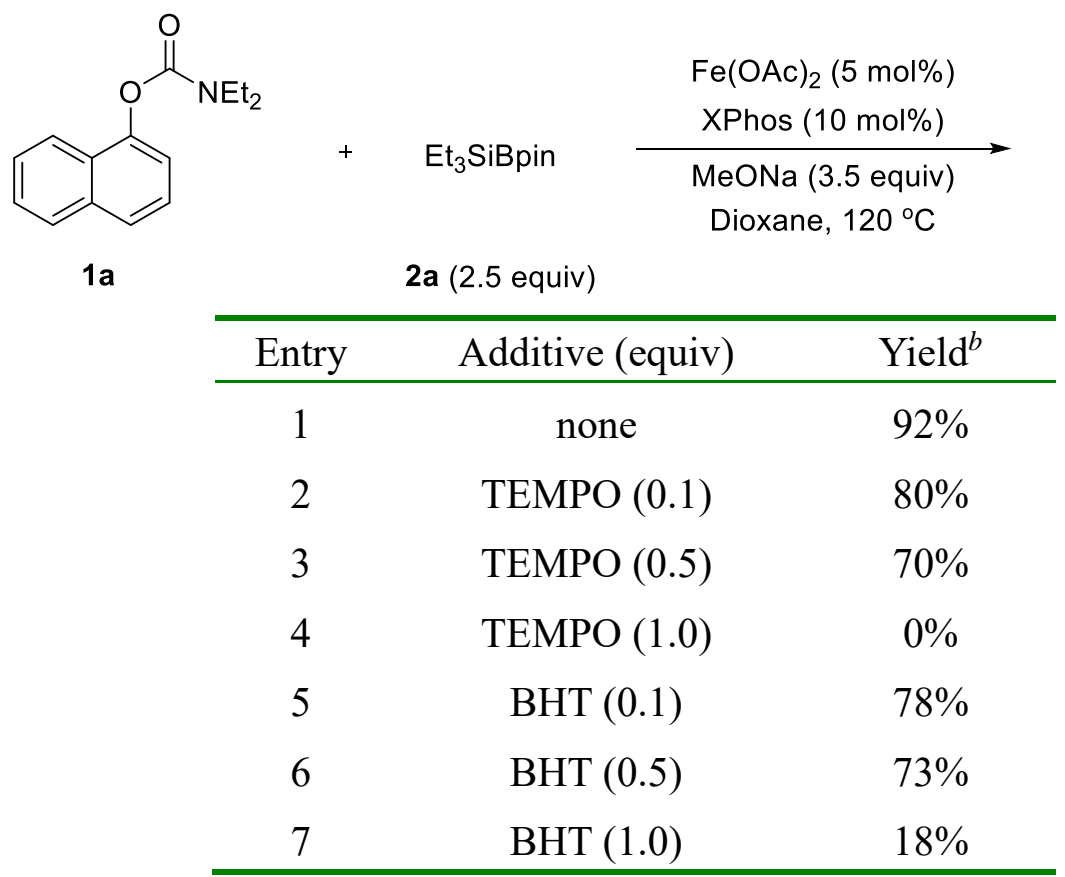


${ }^{a}$ Reaction conditions (unless otherwise specified): $\mathbf{1 a}(0.3 \mathrm{mmol}, 1.0$ equiv), $\mathbf{2 a}$ ( 2.5 equiv), $\mathrm{MeONa}$ (3.5 equiv), dioxane $(1.5 \mathrm{~mL}), 120{ }^{\circ} \mathrm{C}, 15 \mathrm{~h}$, under an argon atmosphere. ${ }^{b}$ Determined by ${ }^{1} \mathrm{H}$ NMR using mesitylene as an internal standard.

Table S13. Radical Inhibition Experiments of Iron-Catalyzed Silylation of 32a ${ }^{a}$

\begin{tabular}{|c|c|c|c|c|}
\hline \multirow[b]{2}{*}{$32 a$} & \multirow[t]{2}{*}{$\mathrm{NMe}_{2}$} & $\mathrm{Et}_{3} \mathrm{Si}-\mathrm{Bpin}$ & \multicolumn{2}{|c|}{$\begin{array}{c}\mathrm{Fe}(\mathrm{OAc})_{2}(10 \mathrm{~mol} \%) \\
\mathrm{MeONa}(4.0 \text { equiv) } \\
\text { Xantphos (12 mol\%) } \\
\underset{\mathrm{MTBE}, 100^{\circ} \mathrm{C}}{\longrightarrow}\end{array}$} \\
\hline & & \multicolumn{3}{|c|}{$2 a$ (2.5 equiv) } \\
\hline & Entry & \multicolumn{2}{|c|}{ Additive (equiv) } & Yield $^{b}$ \\
\hline & 1 & \multicolumn{2}{|c|}{ none } & $90 \%$ \\
\hline & 2 & \multicolumn{2}{|c|}{ TEMPO $(0.1)$} & $45 \%$ \\
\hline & 3 & \multicolumn{2}{|c|}{ TEMPO $(0.5)$} & trace \\
\hline & 4 & \multicolumn{2}{|c|}{ TEMPO (1.0) } & $0 \%$ \\
\hline & 5 & \multicolumn{2}{|c|}{$\operatorname{BHT}(0.1)$} & $50 \%$ \\
\hline & 6 & \multicolumn{2}{|c|}{$\operatorname{BHT}(0.5)$} & trace \\
\hline & 7 & \multicolumn{2}{|c|}{ BHT (1.0) } & $0 \%$ \\
\hline
\end{tabular}

${ }^{a}$ Reaction conditions (unless otherwise specified): 32a $(0.2 \mathrm{mmol}, 1.0$ equiv), 2a (2.5 equiv), $\mathrm{MeONa}$ (4.0 equiv), $\mathrm{Fe}(\mathrm{OAc})_{2}(10 \mathrm{~mol} \%)$, Xantphos (12 mol\%), MTBE $(2.0 \mathrm{~mL}), 100{ }^{\circ} \mathrm{C}, 15 \mathrm{~h}$, under an argon atmosphere. ${ }^{b}$ Determined by ${ }^{1} \mathrm{H}$ NMR using mesitylene as an internal standard.

\section{Radical Clock Experiment}<smiles>CCN(CC)C(=O)Oc1cccc2ccccc12</smiles>

1a<smiles>C=C(c1ccccc1)C1CC1</smiles>

59

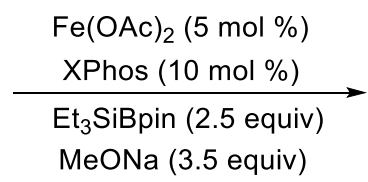

Dioxane, $120^{\circ} \mathrm{C}$<smiles>CCCc1cccc2ccccc12</smiles>

$3,50 \%$

Figure S1. Radical clock experiment

Procedure: To a $25 \mathrm{~mL}$ of Schlenk tube equipped with a magnetic stir bar, 1a $(0.3$ mmol), $\mathrm{Fe}(\mathrm{OAc})_{2}(0.015 \mathrm{mmol}, 3 \mathrm{mg}), \mathrm{XPhos}(0.03 \mathrm{mmol}, 15 \mathrm{mg})$ and $\mathrm{MeONa}(0.75$ mmol, $57 \mathrm{mg}$ ) were added in glove box, followed by $\mathbf{5 9}(0.3 \mathrm{mmol})$, fresh distilled $\mathrm{Et}_{3} \mathrm{SiBpin}(0.75 \mathrm{mmol}, 182 \mathrm{mg})$ and fresh distilled dioxane $(1.5 \mathrm{~mL})$. The reaction mixture was heated at $120^{\circ} \mathrm{C}$ for $15 \mathrm{~h}$. The cooled solution was quenched with saturated $\mathrm{NH}_{4} \mathrm{Cl}$ aqueous solution, then diluted with ethyl acetate and washed with brine. The 
organic phase was dried over $\mathrm{Na}_{2} \mathrm{SO}_{4}$ and concentrated in vacuo. The residue was purified by silica gel flash chromatography to afford the silylation compound $\mathbf{3}$ as a colorless oil (yield 50\%). However, neither radical ring opening product nor product via a plausible $\beta$-carbon elimination pathway was obtained.

\section{EPR Experiments}

Procedure for silylation of aryl carbamate (1a): To a $25 \mathrm{~mL}$ of Schlenk tube equipped with a magnetic stir bar, aryl carbamate 1a $(0.3 \mathrm{mmol}), \mathrm{Fe}(\mathrm{OAc})_{2}(0.015$ mmol, $3 \mathrm{mg}$ ), XPhos (0.03 mmol, $15 \mathrm{mg})$, phenyl tert-butyl nitrone (PBN) (0.3 mmol) and $\mathrm{MeONa}$ (1.05 mmol, $57 \mathrm{mg}$ ) were added in glove box, followed by fresh distilled $\mathrm{Et}_{3} \mathrm{SiBpin}(0.75 \mathrm{mmol}, 182 \mathrm{mg})$ and fresh distilled dioxane $(1.5 \mathrm{~mL})$. The reaction mixture was heated at $120^{\circ} \mathrm{C}$ for $3 \mathrm{~h}$. The resulting mixture was then analyzed by EPR at $120^{\circ} \mathrm{C}$.

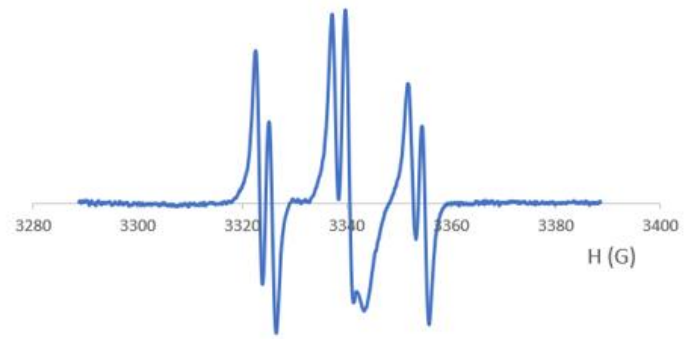

Silylation reaction $\mathrm{g}=2.004928$

Figure S2. EPR experiment of aryl carbamate with phenyl tert-butyl nitrone (PBN) used as spin-trapping reagent.

Procedure for silylation of alkenyl carbamate (32a): To a $25 \mathrm{~mL}$ of Schlenk tube equipped with a magnetic stir bar, alkenyl carbamate 32a $(0.2 \mathrm{mmol})$, Xantphos (0.024 mmol, $13.8 \mathrm{mg})$, phenyl tert-butyl nitrone (PBN) $(0.2 \mathrm{mmol})$ and $\mathrm{MeONa}(0.8 \mathrm{mmol}$, $43.2 \mathrm{mg}$ ) were added in glove box, followed by fresh distilled $\mathrm{Et}_{3} \mathrm{SiBpin}(0.5 \mathrm{mmol}$, $122 \mathrm{mg})$ and fresh distilled methyl tert-butyl ether $(2.0 \mathrm{~mL})$. The reaction mixture was heated at $100{ }^{\circ} \mathrm{C}$ for $3 \mathrm{~h}$. The resulting mixture was then analyzed by EPR at $100{ }^{\circ} \mathrm{C}$. 


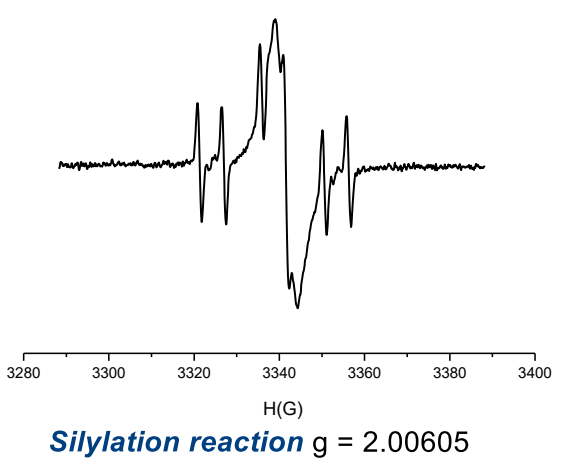

Figure S3. EPR experiment of alkenyl carbamate with phenyl tert-butyl nitrone (PBN) used as spin-trapping reagent.

In conclusion, when $N$-benzylidene-tert-butylamine Noxide (PBN) was introduced into the experiments as a spin-trapping reagent, radical EPR signals were observed. The silylation reaction showed a six-line EPR spectrum $(\mathrm{g}=2.004928$, 2.00605), thus aryl radical might be trapped by PBN to afford EPR active species. ${ }^{1}$

General Procedure for the Synthesis of Aryl Carbamates:

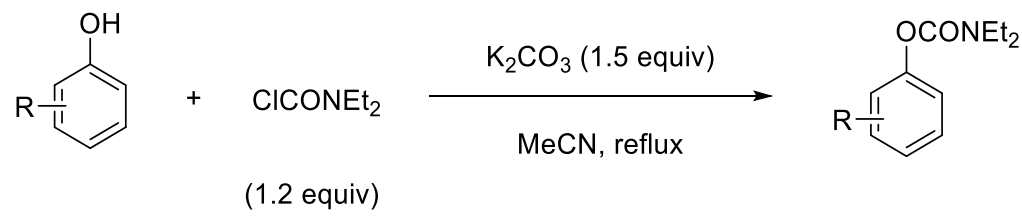

General procedure A: ${ }^{2 \mathrm{a}} \mathrm{A}$ mixture of corresponding phenol (10.0 $\left.\mathrm{mmol}\right), \mathrm{ClCONEt}_{2}$ (1.62 g, $12.0 \mathrm{mmol})$ and $\mathrm{K}_{2} \mathrm{CO}_{3}(2.07 \mathrm{~g}, 15 \mathrm{mmol})$ in $\mathrm{CH}_{3} \mathrm{CN}(25 \mathrm{~mL})$ was refluxed for $8 \mathrm{~h}$. The reaction mixture was cooled to room temperature and concentrated under vacuum. The reaction was quenched with saturated $\mathrm{NaHCO}_{3}$ aqueous solution, then diluted with ethyl acetate and washed with brine. The organic phase was dried over $\mathrm{Na}_{2} \mathrm{SO}_{4}$ and concentrated in vacuo. The residue was purified by silica gel flash chromatography to afford the substrates.<smiles>[R][X]1ccc2c(OCC)cccc2c1</smiles>

(1.2 equiv)

General procedure B: ${ }^{2 b}$ A round bottom flask was charged with $\mathrm{NaH}(0.583 \mathrm{~g}, 14.58$ mmol, 1.2 equiv, $60 \%$ dispersion in oil), and cooled to $0{ }^{\circ} \mathrm{C}$. Then a solution of 2 - 
naphthol (2.0 g, $13.88 \mathrm{mmol}, 1$ equiv) in THF (45 mL) was added dropwise to the $\mathrm{NaH}$. The resulting solution was stirred at $0{ }^{\circ} \mathrm{C}$ for $30 \mathrm{~min}$. A solution of diethyl carbamoyl chloride (1.338 g, $10.76 \mathrm{mmol}, 1.2$ equiv) was then added dropwise to the reaction vessel. The reaction was warmed to room temperature, allowed to stir for $11 \mathrm{~h}$, and then quenched with saturated $\mathrm{NaHCO}_{3}$ aqueous solution, then diluted with ethyl acetate and washed with brine. The organic phase was dried over $\mathrm{Na}_{2} \mathrm{SO}_{4}$ and concentrated in vacuo. The residue was purified by silica gel flash chromatography to afford the substrate.

\section{General Procedure for the Synthesis of Alkenyl Carbamates:}

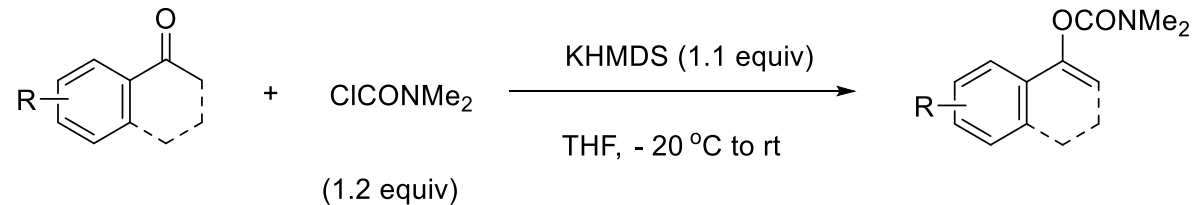

General procedure A (described for the formation of $\mathbf{3 2 a})^{:}{ }^{3}$ To a solution of alphatetralone $(15 \mathrm{mmol})$ in dry THF $(20 \mathrm{~mL})$ at $-20{ }^{\circ} \mathrm{C}$ under argon atmosphere, KHMDS ( $16.5 \mathrm{~mol}, 1 \mathrm{mmol}$ in $1 \mathrm{~mL}$ THF, 1.1 equiv) was added dropwise in $15 \mathrm{~min}$. After stirring for $1 \mathrm{~h}$, dimethylcarbamoyl chloride (1.66 mL, $18.0 \mathrm{mmol}, 1.2$ equiv) was added dropwise in another $15 \mathrm{~min}$. The mixture was warm to $0{ }^{\circ} \mathrm{C}$ and stirring for $30 \mathrm{~min}$, then warm to room temperature. After stirring overnight, the reaction was quenched with saturated $\mathrm{NaHCO}_{3}$ aqueous solution, then diluted with ethyl acetate and washed with brine. The organic phase was dried over $\mathrm{Na}_{2} \mathrm{SO}_{4}$ and concentrated in vacuo. The residue was purified by silica gel flash chromatography $(\mathrm{PE} / \mathrm{EA}=10 / 1)$ to afford the substrate 32a as a colorless solid (yield 60\%-75\%).

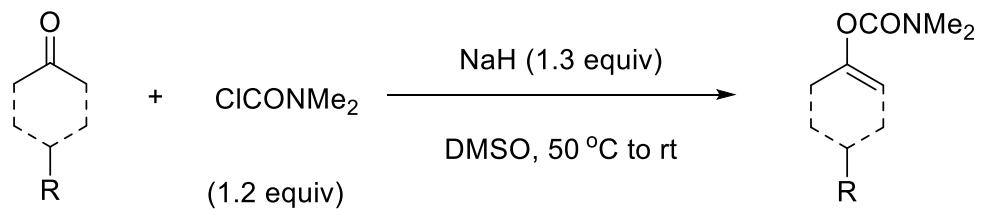

General procedure B (described for the formation of 49a): ${ }^{4}$ The solution of sodium hydride (500 mg, $13.0 \mathrm{mmol}, 1.3$ equiv, $60 \%$ suspension in oil) in dry DMSO (20 mL) at $50{ }^{\circ} \mathrm{C}$ under argon atmosphere was stirred for $2 \mathrm{~h}$ at $50{ }^{\circ} \mathrm{C}$, then the mixture was cooled to room temperature. To the grey solution, 4-phenylcyclohexan-1-one (1.74 g, $10.0 \mathrm{mmol}, 1.0$ equiv) in $5 \mathrm{~mL}$ DMSO was added dropwise in $15 \mathrm{~min}$, the addition being slightly exothermic and changing the color of the solution to yellow. After 15 
min, dimethylcarbamoyl chloride (1.3 mL, $13.0 \mathrm{mmol}, 1.2$ equiv) was added dropwise in another 15 min. And after stirring overnight, saturated $\mathrm{NaHCO}_{3}$ aqueous solution was carefully added to the orange solution, then diluted with ethyl acetate and washed with brine. The organic phase was dried over $\mathrm{Na}_{2} \mathrm{SO}_{4}$ and concentrated in vacuo. The residue was purified by silica gel flash chromatography $(\mathrm{PE} / \mathrm{EA}=10 / 1)$ to afford the substrate 49a as a white solid (yield 30\%-50\%).

\section{General Procedure for the Silylation of Carbamates:}<smiles>CCNC(=O)Oc1cccc2ccccc12</smiles>

$1 \mathrm{a}$
Et 3 Si-Bpin

$2 \mathbf{a}$

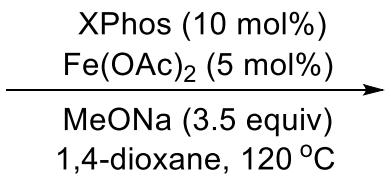

1,4-dioxane, $120^{\circ} \mathrm{C}$<smiles>CCCc1cccc2ccccc12</smiles>

3

Procedure A for the silylation of aryl carbamates (described for typical procedure of the synthesis of 3): A $25 \mathrm{~mL}$ flame-dried Schlenk tube equipped with a magnetic stir bar was charged with $\mathrm{Fe}(\mathrm{OAc})_{2}(0.015 \mathrm{mmol}, 2.6 \mathrm{mg})$, XPhos (0.03 mmol, $14.3 \mathrm{mg}$, 0.1 equiv), MeONa (1.05 mmol, $57 \mathrm{mg}, 3.5$ equiv), naphthalen-1-yl diethylcarbamate 1a (73 mg, $0.3 \mathrm{mmol})$ in glove box. Fresh distilled silylborane 2a $(237 \mathrm{uL}, 0.75 \mathrm{mmol}$, 2.5 equiv), fresh distilled dioxane $(1.5 \mathrm{~mL})$ were then added under argon atmosphere. The reaction mixture was allowed to stir at $120{ }^{\circ} \mathrm{C}$ for $15 \mathrm{~h}$ via heating mantle. The cooled solution was quenched with saturated $\mathrm{NH}_{4} \mathrm{Cl}$ aqueous solution, then diluted with ethyl acetate and washed with brine. The organic phase was dried over $\mathrm{Na}_{2} \mathrm{SO}_{4}$ and concentrated in vacuo. The residue was purified by silica gel flash chromatography with petroleum ether to obtain $65 \mathrm{mg}$ silylated product 3 in $89 \%$ isolated yield. And compounds 4-18 can be obtained in similar procedure.

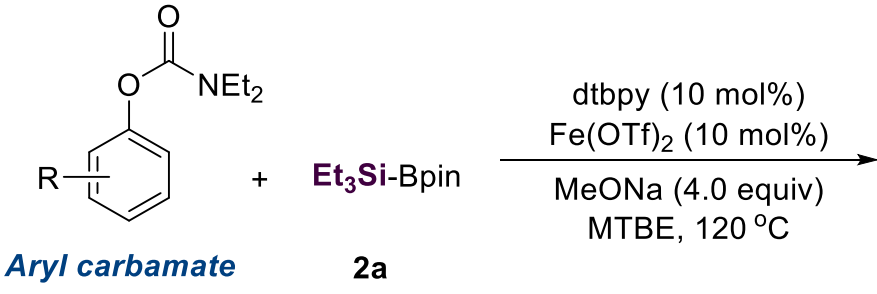<smiles>[R]c1cccc([SiH2]CCCCC)c1</smiles>

Silylated product 
Procedure B for the silylation of aryl carbamates: A $25 \mathrm{~mL}$ flame-dried Schlenk tube equipped with a magnetic stir bar was charged with $\mathrm{Fe}(\mathrm{OTf})_{2}(0.02 \mathrm{mmol}, 7.2 \mathrm{mg}$, 0.1 equiv), dtbpy (0.02 mmol, $7.1 \mathrm{mg}, 0.1$ equiv), $\mathrm{MeONa}(0.8 \mathrm{mmol}, 43.2 \mathrm{mg}, 4.0$ equiv) in glove box. Aryl carbamates $(0.2 \mathrm{mmol})$, fresh distilled silylborane 2a $(0.64$ mmol, 3.2 equiv), fresh distilled methyl tert-butyl ether $(1.5 \mathrm{~mL})$ were then added under argon atmosphere. The reaction mixture was allowed to stir at $120{ }^{\circ} \mathrm{C}$ for $15 \mathrm{~h}$ via heating mantle. The cooled solution was quenched with saturated $\mathrm{NH}_{4} \mathrm{Cl}$ aqueous solution, then diluted with ethyl acetate and washed with brine. The organic phase was dried over $\mathrm{Na}_{2} \mathrm{SO}_{4}$ and concentrated in vacuo. The residue was purified by silica gel flash chromatography with petroleum ether to afford the corresponding compound (1931).<smiles>CNC(=O)OC1=CCCc2ccccc21</smiles>

$32 a$
$+E_{3}$ Si-Bpin

$2 a$

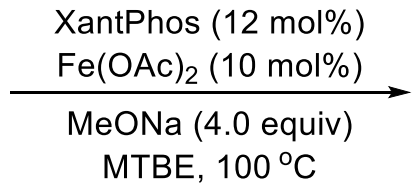

MTBE, $100^{\circ} \mathrm{C}$

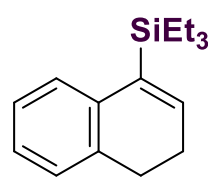

32

Procedure A for the silylation of alkenyl carbamates (described for typical procedure for the synthesis of $\mathbf{3 2}$ on a $1 \mathrm{mmol}$ scale): A $25 \mathrm{~mL}$ flame-dried Schlenk tube equipped with a magnetic stir bar was charged with $\mathrm{Fe}(\mathrm{OAc})_{2}(0.1 \mathrm{mmol}, 17.4 \mathrm{mg}, 0.1$ equiv), Xantphos (0.12 mmol, $69.4 \mathrm{mg}, 0.12$ equiv), MeONa (4.0 mmol, $216.0 \mathrm{mg}, 4.0$ equiv), 3,4-dihydronaphthalen-1-yl dimethylcarbamate 32a (217.1 mg, $1.0 \mathrm{mmol})$ in glove box. Fresh distilled silylborane $\mathbf{2 a}$ (790 uL, $2.5 \mathrm{mmol}, 2.5$ equiv), fresh distilled tert-butyl ether $(10.0 \mathrm{~mL})$ were then added under argon atmosphere. The reaction mixture was allowed to stir at $100{ }^{\circ} \mathrm{C}$ for $15 \mathrm{~h}$ via heating mantle. The cooled solution was quenched with saturated $\mathrm{NH}_{4} \mathrm{Cl}$ aqueous solution, then diluted with ethyl acetate and washed with brine. The organic phase was dried over $\mathrm{Na}_{2} \mathrm{SO}_{4}$ and concentrated in vacuo. The residue was purified by silica gel flash chromatography with petroleum ether to afford 32 in 74\% isolated yield. And compounds 33-48 and 54 can be obtained in similar procedure. 


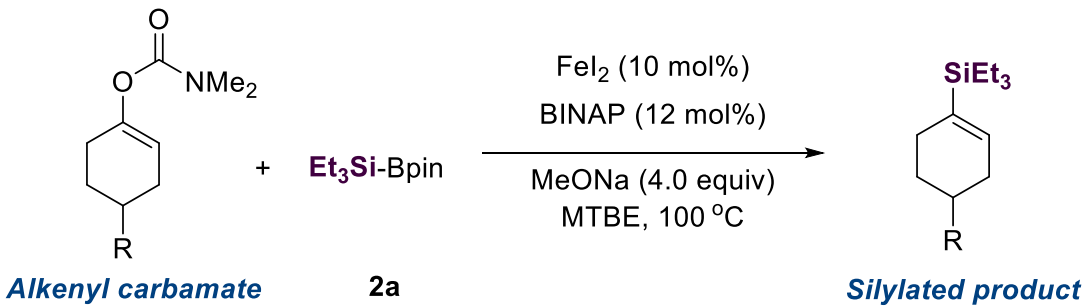

Procedure B for the silylation of alkenyl carbamates: A $25 \mathrm{~mL}$ flame-dried Schlenk tube equipped with a magnetic stir bar was charged with $\mathrm{FeI}_{2}(0.02 \mathrm{mmol}, 6.2 \mathrm{mg}, 0.1$ equiv), BINAP (0.024 mmol, $15 \mathrm{mg}, 0.12$ equiv), $\mathrm{MeONa}(0.8 \mathrm{mmol}, 43.2 \mathrm{mg}, 4.0$ equiv) in glove box. Alkenyl carbamates $(0.2 \mathrm{mmol})$, fresh distilled silylborane $\mathbf{2 a}(0.6$ mmol, 3.0 equiv), fresh distilled tert-butyl ether $(2.0 \mathrm{~mL})$ were then added under argon atmosphere. The reaction mixture was allowed to stir at $100{ }^{\circ} \mathrm{C}$ for $15 \mathrm{~h}$ via heating mantle. The cooled solution was quenched with saturated $\mathrm{NH}_{4} \mathrm{Cl}$ aqueous solution, then diluted with ethyl acetate and washed with brine. The organic phase was dried over $\mathrm{Na}_{2} \mathrm{SO}_{4}$ and concentrated in vacuo. The residue was purified by silica gel flash chromatography with petroleum ether to afford the corresponding compound (49-53). 


\section{Experimental Procedures and Characterization Data for Silanes Products}<smiles>CCCc1cccc2ccccc12</smiles>

Triethyl(naphthalen-1-yl)silane (3). This compound is known. ${ }^{5}$ The product $\mathbf{3}(65 \mathrm{mg}$, $89 \%$ yield) as a colourless oil was purified with silica gel chromatography (Petroleum ether). ${ }^{1} \mathrm{H}$ NMR $\left(400 \mathrm{MHz}, \mathrm{CDCl}_{3}\right) \delta$ 8.16-8.13 (m, $\left.1 \mathrm{H}\right)$, 7.90-7.88 (m, $\left.2 \mathrm{H}\right), 7.72(\mathrm{~d}$, $J=6.8 \mathrm{~Hz}, 1 \mathrm{H}), 7.55-7.47(\mathrm{~m}, 3 \mathrm{H}), 1.10-0.97(\mathrm{~m}, 15 \mathrm{H}) .{ }^{13} \mathrm{C} \mathrm{NMR}\left(100 \mathrm{MHz}, \mathrm{CDCl}_{3}\right)$ $\delta 137.5,135.2,134.5,133.4,129.6,129.1,127.8,125.5,125.2,125.1,7.7,4.6$. HRMS (EI/TOF): Calculated for $\mathrm{C}_{16} \mathrm{H}_{22} \mathrm{Si}\left(\mathrm{M}^{+}\right)$: 242.1491; Found: 242.1482.<smiles>CC[SiH2]c1ccc2ccccc2c1</smiles>

Triethyl(naphthalen-2-yl)silane (4). This compound is known. ${ }^{5}$ The product 4 (69 mg, 94\% yield) as a colourless oil was purified with silica gel chromatography (Petroleum ether). ${ }^{1} \mathrm{H}$ NMR $\left(400 \mathrm{MHz}, \mathrm{CDCl}_{3}\right) \delta 8.02(\mathrm{~s}, 1 \mathrm{H})$, 7.89-7.83 (m, $\left.3 \mathrm{H}\right)$, 7.61-7.59 (m, $1 \mathrm{H}), 7.52-7.47$ (m, $2 \mathrm{H}), 1.06-1.01$ (m, $9 \mathrm{H}), 0.94-0.88$ (m, $6 \mathrm{H}) .{ }^{13} \mathrm{C}$ NMR (100 MHz, $\left.\mathrm{CDCl}_{3}\right) \delta 135.0,134.8,133.6,132.9,130.6,128.0,127.7,126.7,126.1,125.7,7.5,3.4$. HRMS (EI/TOF): Calculated for $\mathrm{C}_{16} \mathrm{H}_{22} \mathrm{Si}\left(\mathrm{M}^{+}\right)$: 242.1491; Found: 242.1495 .

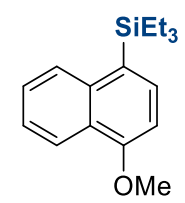

Triethyl(4-methoxynaphthalen-1-yl)silane (5). This compound is known. ${ }^{5}$ The product 5 (56 mg, 68\% yield) was purified with silica gel chromatography (Petroleum ether). ${ }^{1} \mathrm{H}$ NMR $\left(400 \mathrm{MHz}, \mathrm{CDCl}_{3}\right) \delta 8.38-8.35(\mathrm{~m}, 1 \mathrm{H}), 8.08-8.06(\mathrm{~m}, 1 \mathrm{H}), 7.61(\mathrm{~d}$, $J=7.7 \mathrm{~Hz}, 1 \mathrm{H}), 7.56-7.43$ (m, $2 \mathrm{H}), 6.83(\mathrm{~d}, J=7.7 \mathrm{~Hz}, 1 \mathrm{H}), 4.02(\mathrm{~s}, 3 \mathrm{H}), 1.07-0.98$ $(\mathrm{m}, 15 \mathrm{H}) .{ }^{13} \mathrm{C} \mathrm{NMR}\left(100 \mathrm{MHz}, \mathrm{CDCl}_{3}\right) \delta 156.5,138.5,134.9,127.6,126.1,125.8$, 125.7, 124.6, 122.6, 123.2, 55.3, 7.7, 4.5. HRMS (EI/TOF): Calculated for $\mathrm{C}_{17} \mathrm{H}_{24} \mathrm{OSi}$ $\left(\mathrm{M}^{+}\right)$: 272.1596; Found: 272.1600 
$\mathrm{SiEt}_{3}$

Triethyl(7-methoxynaphthalen-2-yl)silane (6). This compound is known. ${ }^{5}$ The product 6 (60 mg, 74\% yield) as a colourless oil was purified with silica gel chromatography (Petroleum ether). ${ }^{1} \mathrm{H}$ NMR (400 MHz, $\left.\mathrm{CDCl}_{3}\right) \delta 7.91$ (s, $\left.1 \mathrm{H}\right), 7.75$ (t, $J=9.3 \mathrm{~Hz}, 2 \mathrm{H}), 7.45(\mathrm{~d}, J=8.1 \mathrm{~Hz}, 1 \mathrm{H}), 7.18-7.15(\mathrm{~m}, 2 \mathrm{H}), 3.95$ (s, $3 \mathrm{H}), 1.05-$ $1.01(\mathrm{~m}, 9 \mathrm{H}), 0.93-0.87(\mathrm{~m}, 6 \mathrm{H}) .{ }^{13} \mathrm{C} \mathrm{NMR}\left(100 \mathrm{MHz}, \mathrm{CDCl}_{3}\right) \delta 157.5,135.4,134.0$, 133.5, 129.2, 128.5, 126.5, 119.0, 105.8, 55.3, 7.5, 3.4. HRMS (EI/TOF): Calculated for $\mathrm{C}_{17} \mathrm{H}_{24} \mathrm{OSi}\left(\mathrm{M}^{+}\right)$: 272.1596; Found: 272.1597.<smiles>CCCCC(C)Cc1ccc2cc(OC(C)C)ccc2c1</smiles>

tert-Butyldimethyl((6-(triethylsilyl)naphthalen-2-yl)oxy)silane (7). The product 7 (79 mg, 71\% yield) as a colourless oil was purified with silica gel chromatography (Petroleum ether). ${ }^{1} \mathrm{H}$ NMR (400 MHz, $\left.\mathrm{CDCl}_{3}\right) \delta 7.84$ (s, $\left.1 \mathrm{H}\right)$, 7.76-7.70 (m, $\left.2 \mathrm{H}\right), 7.44$ $(\mathrm{d}, J=8.1 \mathrm{~Hz}, 1 \mathrm{H}), 7.21(\mathrm{~d}, J=2.4 \mathrm{~Hz}, 1 \mathrm{H}), 7.09(\mathrm{dd}, J=8.9,2.4 \mathrm{~Hz}, 1 \mathrm{H}), 1.05-1.00$ $(\mathrm{m}, 18 \mathrm{H}), 0.92-0.95(\mathrm{~m}, 6 \mathrm{H}), 0.27(\mathrm{~s}, 6 \mathrm{H}) .{ }^{13} \mathrm{C} \mathrm{NMR}\left(100 \mathrm{MHz}, \mathrm{CDCl}_{3}\right) \delta 153.3$, 135.2, 134.1, 133.5, 129.4, 129.0, 128.6, 126.4, 122.4, 115.0, 25.7, 18.3, 7.5, 3.4, -4.3. HRMS (EI/TOF): Calculated for $\mathrm{C}_{22} \mathrm{H}_{36} \mathrm{OSi}_{2}\left(\mathrm{M}^{+}\right)$: 372.2305 ; Found: 372.2307 .

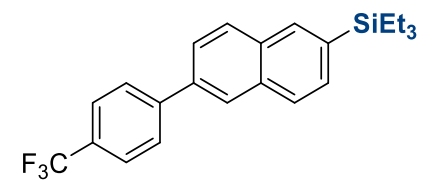

Triethyl(6-(4-(trifluoromethyl)phenyl)naphthalen-2-yl)silane (8). The product 8 (101 mg, 87\% yield, m.p. $48.5-50.1{ }^{\circ} \mathrm{C}$ ) as a white solid was purified with silica gel chromatography (Petroleum ether). ${ }^{1} \mathrm{H}$ NMR (400 MHz, $\left.\mathrm{CDCl}_{3}\right) \delta$ 8.05-8.03 (m, $\left.2 \mathrm{H}\right)$, $7.95(\mathrm{~d}, J=8.8 \mathrm{~Hz}, 1 \mathrm{H}), 7.89(\mathrm{~d}, J=8.2 \mathrm{~Hz}, 1 \mathrm{H}), 7.83(\mathrm{~d}, J=8.2 \mathrm{~Hz}, 2 \mathrm{H}), 7.77-7.71$ $(\mathrm{m}, 3 \mathrm{H}), 7.66-7.62(\mathrm{~m}, 1 \mathrm{H}), 1.06-0.99(\mathrm{~m}, 9 \mathrm{H}), 0.95-0.86(\mathrm{~m}, 6 \mathrm{H}) .{ }^{13} \mathrm{C}$ NMR $(100$ $\left.\mathrm{MHz}, \mathrm{CDCl}_{3}\right) \delta 144.7,137.3,136.0,134.5,133.7,132.4,131.4,129.0\left(\mathrm{q},{ }^{2} J_{\mathrm{CF}}=32.0\right.$ $\mathrm{Hz}), 128.9,127.6,127.1,126.1,125.8\left(\mathrm{q},{ }^{3} J_{\mathrm{CF}}=3.9 \mathrm{~Hz}\right), 125.0,7.4,3.4 . \mathrm{HRMS}$ (EI/TOF): Calculated for $\mathrm{C}_{23} \mathrm{H}_{25} \mathrm{~F}_{3} \mathrm{Si}\left(\mathrm{M}^{+}\right)$: 386.1678; Found: 386.1671 . 
<smiles>CCCc1ccc2cc(-c3ccc(C)cc3)ccc2c1</smiles>

Triethyl(6-(p-tolyl)naphthalen-2-yl)silane (9). The product 9 (70 mg, 72\% yield) was purified with silica gel chromatography (Petroleum ether). ${ }^{1} \mathrm{H}$ NMR $\left(400 \mathrm{MHz}, \mathrm{CDCl}_{3}\right)$ $\delta 8.04(\mathrm{~m}, 2 \mathrm{H}), 7.93(\mathrm{~d}, J=8.0 \mathrm{~Hz}, 1 \mathrm{H}), 7.89(\mathrm{~d}, J=8.0 \mathrm{~Hz}, 1 \mathrm{H}), 7.77(\mathrm{~d}, J=8.0 \mathrm{~Hz}$, $1 \mathrm{H}), 7.66(\mathrm{~d}, J=8.0 \mathrm{~Hz}, 2 \mathrm{H}), 7.63(\mathrm{~d}, J=8.0 \mathrm{~Hz}, 1 \mathrm{H}), 7.33(\mathrm{~d}, J=8.0 \mathrm{~Hz}, 2 \mathrm{H}), 2.45$ $(\mathrm{s}, 3 \mathrm{H}), 1.07-1.03(\mathrm{~m}, 9 \mathrm{H}), 0.96-0.89(\mathrm{~m}, 6 \mathrm{H}) .{ }^{13} \mathrm{C} \mathrm{NMR}\left(100 \mathrm{MHz}, \mathrm{CDCl}_{3}\right) \delta 138.7$, $138.3,137.1,134.9,134.5,133.9,132.0,131.0,129.6,128.4,127.2,127.0,125.4,125.2$, 21.1, 7.5, 3.4. HRMS (EI/TOF): Calculated for $\mathrm{C}_{23} \mathrm{H}_{28} \mathrm{Si}\left(\mathrm{M}^{+}\right)$: 332.1960; Found: 332.1966.

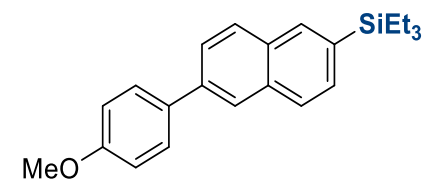

Triethyl(6-(4-methoxyphenyl)naphthalen-2-yl)silane (10). The product 10 (93 mg, 89\% yield, m.p. 103.0-104.6 ${ }^{\circ} \mathrm{C}$ ) as a white solid was purified with silica gel chromatography (Petroleum ether). ${ }^{1} \mathrm{H}$ NMR $\left(400 \mathrm{MHz}, \mathrm{CDCl}_{3}\right) \delta 8.02(\mathrm{~s}, 1 \mathrm{H}), 8.00$ $(\mathrm{s}, 1 \mathrm{H}), 7.92(\mathrm{~d}, J=8.0 \mathrm{~Hz}, 1 \mathrm{H}), 7.88(\mathrm{~d}, J=8.0 \mathrm{~Hz}, 1 \mathrm{H}), 7.76(\mathrm{~d}, J=8.0 \mathrm{~Hz}, 1 \mathrm{H})$, $7.69(\mathrm{~d}, J=8.7 \mathrm{~Hz}, 2 \mathrm{H}), 7.62(\mathrm{~d}, J=8.1 \mathrm{~Hz}, 1 \mathrm{H}), 7.05(\mathrm{~d}, J=8.7 \mathrm{~Hz}, 2 \mathrm{H}), 3.89$ (s, 3 $\mathrm{H}), 1.07-1.03(\mathrm{~m}, 9 \mathrm{H}), 0.95-0.89(\mathrm{~m}, 6 \mathrm{H}) .{ }^{13} \mathrm{C} \mathrm{NMR}\left(100 \mathrm{MHz}, \mathrm{CDCl}_{3}\right) \delta$ 159.2, 138.4, $134.8,134.5,133.9,133.6,131.8,131.0,128.5,128.4,126.9,125.3,124.8,114.3,55.3$, 7.5, 3.4. HRMS (EI/TOF): Calculated for $\mathrm{C}_{23} \mathrm{H}_{28} \mathrm{OSi}\left(\mathrm{M}^{+}\right)$: 348.1909; Found: 348.1914.<smiles>CC[SiH2]c1ccc2cc3ccccc3cc2c1</smiles>

Anthracen-2-yltriethylsilane (11). This compound is known. ${ }^{6}$ The product 11 (62 mg, $71 \%$ yield, m.p. $102.4-105.4{ }^{\circ} \mathrm{C}$ ) as a white solid was purified with silica gel chromatography (Petroleum ether). ${ }^{1} \mathrm{H}$ NMR (400 MHz, $\left.\mathrm{CDCl}_{3}\right) \delta 8.43(\mathrm{~s}, 1 \mathrm{H}), 8.40$ (s, $1 \mathrm{H}), 8.16(\mathrm{~s}, 1 \mathrm{H}), 8.03-7.99(\mathrm{~m}, 2 \mathrm{H}), 7.97(\mathrm{~d}, J=8.0 \mathrm{~Hz}, 1 \mathrm{H}), 7.54(\mathrm{~d}, J=8.0 \mathrm{~Hz}$, $1 \mathrm{H}), 7.48-7.44(\mathrm{~m}, 2 \mathrm{H}), 1.07-1.03(\mathrm{t}, J=7.5 \mathrm{~Hz}, 9 \mathrm{H}), 0.94-0.88(\mathrm{~m}, 6 \mathrm{H}) .{ }^{13} \mathrm{C}$ NMR $\left(100 \mathrm{MHz}, \mathrm{CDCl}_{3}\right) \delta 135.4,134.6,131.9,131.7,131.3,129.6,128.3,128.1,126.8$, 
126.3, 125.9, 125.3, 125.3, 7.5, 3.3. FTMS (ESI/quadrupole): Calculated for $\mathrm{C}_{20} \mathrm{H}_{25} \mathrm{Si}$ $(\mathrm{M}+\mathrm{H})^{+}:$293.1720; Found: 293.1726.

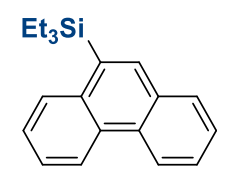

Triethyl(phenanthren-9-yl)silane (12). The product $12(58 \mathrm{mg}, 66 \%$ yield) as a colourless oil was purified with silica gel chromatography (Petroleum ether). ${ }^{1} \mathrm{H}$ NMR $\left(400 \mathrm{MHz}, \mathrm{CDCl}_{3}\right) \delta 8.75(\mathrm{~d}, J=8.0 \mathrm{~Hz}, 1 \mathrm{H}), 8.68(\mathrm{~d}, J=8.0 \mathrm{~Hz}, 1 \mathrm{H}), 8.15(\mathrm{~d}, J=$ $7.8 \mathrm{~Hz}, 1 \mathrm{H}), 7.94$ (s, $1 \mathrm{H}), 7.89$ (d, $J=8.0 \mathrm{~Hz}, 1 \mathrm{H}), 7.69-7.59$ (m, $4 \mathrm{H}), 1.09-0.98$ (m, $15 \mathrm{H}) .{ }^{13} \mathrm{C}$ NMR $\left(100 \mathrm{MHz}, \mathrm{CDCl}_{3}\right) \delta 136.6,135.5,133.7,131.1,130.9,129.9,128.8$, 128.5, 127.0, 126.5, 126.2, 125.9, 123.2, 122.4, 7.7, 4.6. HRMS (EI/TOF): Calculated for $\mathrm{C}_{20} \mathrm{H}_{24} \mathrm{Si}\left(\mathrm{M}^{+}\right)$: 292.1647; Found: 292.1642 .

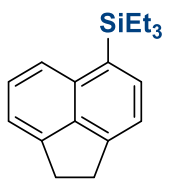

(1,2-Dihydroacenaphthylen-5-yl)triethylsilane (13). The product 13 (59 mg, 74\% yield) as a colourless oil was purified with silica gel chromatography (Petroleum ether). ${ }^{1} \mathrm{H} \mathrm{NMR}\left(400 \mathrm{MHz}, \mathrm{CDCl}_{3}\right) \delta 7.90(\mathrm{~d}, J=8.0 \mathrm{~Hz}, 1 \mathrm{H}), 7.71(\mathrm{~d}, J=6.8 \mathrm{~Hz}, 1 \mathrm{H}), 7.53$ $(\mathrm{d}, J=6.8 \mathrm{~Hz}, 1 \mathrm{H}), 7.38-7.35(\mathrm{~m}, 2 \mathrm{H}), 3.45(\mathrm{~m}, 4 \mathrm{H}), 1.09-1.07(\mathrm{~m}, 15 \mathrm{H}) .{ }^{13} \mathrm{C} \mathrm{NMR}$ $\left(100 \mathrm{MHz}, \mathrm{CDCl}_{3}\right) \delta 147.7,146.9,139.0,136.0,129.6,127.5,123.1,119.1,30.24$, 30.21, 7.8, 4.5. HRMS (EI/TOF): Calculated for $\mathrm{C}_{18} \mathrm{H}_{24} \mathrm{Si}\left(\mathrm{M}^{+}\right)$: 268.1647; Found: 268.1649 .

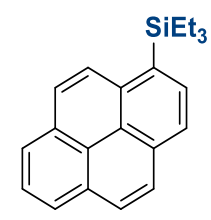

Triethyl(pyren-1-yl)silane (14). The product 14 (77 mg, 81\% yield, m.p. 58.2-59.8 $\left.{ }^{\circ} \mathrm{C}\right)$ as a white solid was purified with silica gel chromatography (Petroleum ether). ${ }^{1} \mathrm{H}$ NMR (400 MHz, $\left.\mathrm{CDCl}_{3}\right) \delta 8.40(\mathrm{~d}, J=9.6 \mathrm{~Hz}, 1 \mathrm{H}), 8.23-8.16(\mathrm{~m}, 4 \mathrm{H}), 8.13(\mathrm{~m}, 1 \mathrm{H})$, 
8.10-8.06 (m, $2 \mathrm{H}), 8.05-7.98(\mathrm{~m}, 1 \mathrm{H}), 1.20-1.15(\mathrm{~m}, 6 \mathrm{H}), 1.07-1.01(\mathrm{~m}, 9 \mathrm{H}) .{ }^{13} \mathrm{C}$ $\mathrm{NMR}\left(100 \mathrm{MHz}, \mathrm{CDCl}_{3}\right) \delta 136.2,133.2,132.8,131.9,131.3,131.1,130.6,127.7,127.5$, 127.4, 126.9, 125.7, 125.0, 124.9, 124.6, 124.0, 7.8, 4.9. HRMS (EI/TOF): Calculated for $\mathrm{C}_{22} \mathrm{H}_{24} \mathrm{Si}\left(\mathrm{M}^{+}\right)$: 316.1647; Found: 316.1654 .

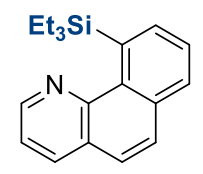

10-(Triethylsilyl)benzo[H]quinoline (15). This compound is known. ${ }^{7}$ The product $\mathbf{1 5}$ (40 mg, 45\% yield) as a light yellow oil was purified with silica gel chromatography (Petroleum ether). ${ }^{1} \mathrm{H}$ NMR (400 MHz, $\left.\mathrm{CDCl}_{3}\right) \delta 8.95(\mathrm{~m}, 1 \mathrm{H}), 8.19(\mathrm{~d}, J=8.0 \mathrm{~Hz}, 1$ H), 8.07 (d, $J=7.2 \mathrm{~Hz}, 1 \mathrm{H}), 7.94(\mathrm{~d}, J=7.9 \mathrm{~Hz}, 1 \mathrm{H}), 7.84$ (d, $J=7.9 \mathrm{~Hz}, 1 \mathrm{H}), 7.71$ (m, $1 \mathrm{H}), 7.69-7.67(\mathrm{~m}, 1 \mathrm{H}), 7.53(\mathrm{~m}, 1 \mathrm{H}), 1.03(\mathrm{q}, J=7.7 \mathrm{~Hz}, 6 \mathrm{H}), 0.91(\mathrm{t}, J=7.7$ $\mathrm{Hz}, 9 \mathrm{H}) .{ }^{13} \mathrm{C} \mathrm{NMR}\left(100 \mathrm{MHz}, \mathrm{CDCl}_{3}\right) \delta 147.1,145.7,137.0,136.8,135.5,135.3,133.8$, 129.2, 129.0, 127.2, 126.5, 124.6, 121.4, 8.6, 7.0. FTMS (ESI/Quadrupole): Calculated for $\mathrm{C}_{19} \mathrm{H}_{24} \mathrm{NSi}(\mathrm{M}+\mathrm{H})^{+}:$294.1673; Found: 294.1669 .

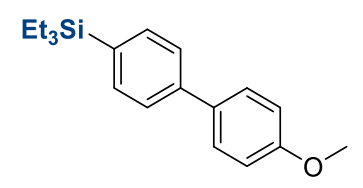

Triethyl(4'-methoxy-[1,1'-biphenyl]-4-yl)silane (16). This compound is known. ${ }^{8}$ The product $16\left(57 \mathrm{mg}, 64 \%\right.$ yield, m.p. $\left.61.2-64.0^{\circ} \mathrm{C}\right)$ as a white solid was purified with silica gel chromatography (Petroleum ether). ${ }^{1} \mathrm{H} \mathrm{NMR}\left(400 \mathrm{MHz}, \mathrm{CDCl}_{3}\right) \delta$ 7.57-7.52 (m, $6 \mathrm{H}), 6.98(\mathrm{~d}, J=8.4 \mathrm{~Hz}, 2 \mathrm{H}), 3.86(\mathrm{~s}, 3 \mathrm{H}), 1.00(\mathrm{t}, J=7.9 \mathrm{~Hz}, 9 \mathrm{H}), 0.85-0.79$ (m, $6 \mathrm{H}) .{ }^{13} \mathrm{C} \mathrm{NMR}\left(100 \mathrm{MHz}, \mathrm{CDCl}_{3}\right) \delta 159.2,141.0,135.4,134.6,133.7,128.1,125.9$, 114.2, 55.3, 7.4, 3.4. HRMS (EI/TOF): Calculated for $\mathrm{C}_{19} \mathrm{H}_{26} \mathrm{OSi}\left(\mathrm{M}^{+}\right)$: 298.1753; Found: 298.1761.

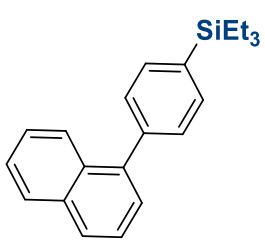


Triethyl(4-(naphthalen-1-yl)phenyl)silane (17). The product 17 (54 mg, 57\% yield) was purified with silica gel chromatography (Petroleum ether). ${ }^{1} \mathrm{H}$ NMR (400 MHz, $\left.\mathrm{CDCl}_{3}\right) \delta 7.96(\mathrm{~d}, J=8.4 \mathrm{~Hz}, 1 \mathrm{H}), 7.92(\mathrm{~d}, J=8.4 \mathrm{~Hz}, 1 \mathrm{H}), 7.87(\mathrm{~d}, J=8.4 \mathrm{~Hz}, 1 \mathrm{H})$, 7.64-6.62 (m, $2 \mathrm{H}), 7.54-7.45(\mathrm{~m}, 6 \mathrm{H}), 1.08-1.03(\mathrm{~m}, 9 \mathrm{H}), 092-0.85(\mathrm{~m}, 6 \mathrm{H}) .{ }^{13} \mathrm{C}$ NMR (100 MHz, $\left.\mathrm{CDCl}_{3}\right): \delta 141.0,140.3,136.2,134.1,133.8,131.5,130.0,129.3$, 128.2, 127.6, 126.9, 126.1, 126.0, 125.9, 125.7, 125.4, 7.5, 3.4. HRMS (EI/TOF): Calculated for $\mathrm{C}_{22} \mathrm{H}_{26} \mathrm{Si}\left(\mathrm{M}^{+}\right)$: 318.1804; Found: 318.1814 .

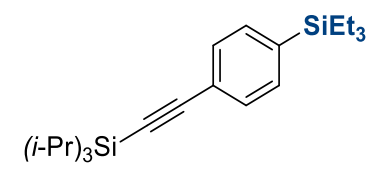

Triethyl(4-((triisopropylsilyl)ethynyl)phenyl)silane (18). The product 18 (90 $\mathrm{mg}, 81 \%$ yield) as a colourless oil was purified with silica gel chromatography (Petroleum ether). ${ }^{1} \mathrm{H}$ NMR (400 MHz, $\left.\mathrm{CDCl}_{3}\right) \delta$ 7.48-7.41 (m, $\left.4 \mathrm{H}\right), 1.14-1.13(\mathrm{~m}, 21 \mathrm{H}), 0.95(\mathrm{t}, J=7.6$ $\mathrm{Hz}, 9 \mathrm{H}), 0.80-0.75$ (m, $6 \mathrm{H}) .{ }^{13} \mathrm{C} \mathrm{NMR}\left(100 \mathrm{MHz}, \mathrm{CDCl}_{3}\right) \delta 138.1,133.9,131.0,123.7$, 94.2, 90.9, 18.7, 11.3, 7.3, 3.2. HRMS (EI/TOF): Calculated for $\mathrm{C}_{23} \mathrm{H}_{40} \mathrm{Si}_{2}\left(\mathrm{M}^{+}\right)$: 372.2669; Found: 372.2668.<smiles>CC[Si]c1ccc(C(C)(C)C)cc1</smiles>

(4-(tert-Butyl)phenyl)triethylsilane (19). This compound is known. ${ }^{9}$ The reaction was carried out according to the general procedure on $0.2 \mathrm{mmol}$ scale, and $10 \mathrm{~mol} \%$ $\mathrm{Fe}(\mathrm{OTf})_{2}, 10 \mathrm{~mol} \%$ dtbpy was used. The product 19 (34 mg, 69\% yield) as a colourless oil was purified with silica gel chromatography (Petroleum ether). ${ }^{1} \mathrm{H}$ NMR (600 MHz, $\left.\mathrm{CDCl}_{3}\right) \delta 7.45(\mathrm{~d}, J=7.6 \mathrm{~Hz}, 2 \mathrm{H}), 7.38(\mathrm{~d}, J=7.6 \mathrm{~Hz}, 2 \mathrm{H}), 1.34(\mathrm{~s}, 9 \mathrm{H}), 0.99(\mathrm{t}, J=$ $7.8 \mathrm{~Hz}, 9 \mathrm{H}), 0.80(\mathrm{q}, J=7.8 \mathrm{~Hz}, 6 \mathrm{H}) .{ }^{13} \mathrm{C} \mathrm{NMR}\left(125 \mathrm{MHz}, \mathrm{CDCl}_{3}\right) \delta 151.5,134.0$, 133.8, 124.6, 34.6, 31.3, 7.5, 3.4. HRMS (EI/TOF): Calculated for $\mathrm{C}_{16} \mathrm{H}_{28} \mathrm{Si}\left(\mathrm{M}^{+}\right)$: 248.1960; Found: 248.1959.

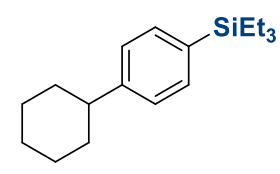


(4-Cyclohexylphenyl)triethylsilane (20). The reaction was carried out according to the general procedure on $0.2 \mathrm{mmol}$ scale, and $10 \% \mathrm{Fe}(\mathrm{OTf})_{2}, 10 \%$ dtbpy was used. The product 20 (29 mg, 53\% yield) was purified with silica gel chromatography (Petroleum ether). ${ }^{1} \mathrm{H}$ NMR $\left(600 \mathrm{MHz}, \mathrm{CDCl}_{3}\right) \delta 7.43(\mathrm{~d}, J=7.6 \mathrm{~Hz}, 2 \mathrm{H}), 7.21(\mathrm{~d}, J=7.6 \mathrm{~Hz}, 2$ H), $2.50(\mathrm{t}, J=11.3 \mathrm{~Hz}, 1 \mathrm{H}), 1.95-1.81(\mathrm{~m}, 4 \mathrm{H}), 1.80-1.73(\mathrm{~m}, 1 \mathrm{H}), 1.50-1.35(\mathrm{~m}, 5$ $\mathrm{H}), 0.99(\mathrm{t}, J=7.9 \mathrm{~Hz}, 9 \mathrm{H}), 0.80(\mathrm{q}, J=7.9 \mathrm{~Hz}, 6 \mathrm{H}) .{ }^{13} \mathrm{C} \mathrm{NMR}\left(100 \mathrm{MHz}, \mathrm{CDCl}_{3}\right): \delta$ 148.5, 134.2, 126.2, 44.5, 34.3, 26.9, 26.2, 7.5, 3.4. FTMS (EI/Orbitrap): Calculated for $\mathrm{C}_{18} \mathrm{H}_{30} \mathrm{Si}\left(\mathrm{M}^{+}\right):$274.2116; Found: 274.2117.

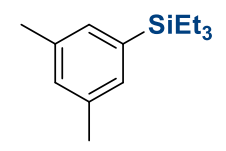

(3,5-Dimethylphenyl)triethylsilane (21). This compound is known. ${ }^{10}$ The reaction was carried out according to the general procedure on $0.2 \mathrm{mmol} \mathrm{scale}$, and $10 \mathrm{~mol} \%$ $\mathrm{Fe}(\mathrm{OTf})_{2}, 10 \mathrm{~mol} \%$ dtbpy was used. The product 21 (30 mg, 68\% yield) as a colourless oil was purified with silica gel chromatography (Petroleum ether). ${ }^{1} \mathrm{H}$ NMR $(600 \mathrm{MHz}$, $\left.\mathrm{CDCl}_{3}\right) \delta 7.11(\mathrm{~s}, 2 \mathrm{H}), 7.01(\mathrm{~s}, 1 \mathrm{H}), 2.34(\mathrm{~s}, 6 \mathrm{H}), 0.99(\mathrm{t}, J=7.8 \mathrm{~Hz}, 9 \mathrm{H}), 0.80(\mathrm{q}, J$ $=7.8 \mathrm{~Hz}, 6 \mathrm{H}) .{ }^{13} \mathrm{C} \mathrm{NMR}\left(125 \mathrm{MHz}, \mathrm{CDCl}_{3}\right) \delta 137.2,136.8,131.9,130.5,21.4,7.5$, 3.4. HRMS (EI/TOF): Calculated for $\mathrm{C}_{14} \mathrm{H}_{24} \mathrm{Si}\left(\mathrm{M}^{+}\right)$: 220.1647; Found: 220.1644 .<smiles>CCSc1ccc2c(c1)CCCC2</smiles>

Triethyl(5,6,7,8-tetrahydronaphthalen-2-yl)silane (22). The reaction was carried out according to the general procedure on $0.2 \mathrm{mmol}$ scale, and $10 \% \mathrm{Fe}(\mathrm{OTf})_{2}, 10 \%$ dtbpy was used. The product $22(25 \mathrm{mg}, 51 \%$ yield $)$ was purified with silica gel chromatography (Petroleum ether). ${ }^{1} \mathrm{H} \mathrm{NMR}\left(600 \mathrm{MHz}, \mathrm{CDCl}_{3}\right) \delta 7.23$ (d, J=7.4 Hz, $1 \mathrm{H}), 7.20$ (s, $1 \mathrm{H}), 7.07$ (d, $J=7.4 \mathrm{~Hz}, 1 \mathrm{H}), 2.83-2.74(\mathrm{~m}, 4 \mathrm{H}), 1.87-1.78(\mathrm{~m}, 4 \mathrm{H})$, 0.99 (t, $J=7.9 \mathrm{~Hz}, 9 \mathrm{H}), 0.79$ (q, $J=7.9 \mathrm{~Hz}, 6 \mathrm{H}) .{ }^{13} \mathrm{C} \mathrm{NMR}\left(100 \mathrm{MHz}, \mathrm{CDCl}_{3}\right): \delta 137.8$, 136.3, 135.2, 133.9, 131.2, 128.5, 29.4, 23.2, 7.5, 3.4. FTMS (EI/Orbitrap): Calculated for $\mathrm{C}_{16} \mathrm{H}_{26} \mathrm{Si}\left(\mathrm{M}^{+}\right)$: 246.1804; Found: 246.1803. 
<smiles>CC[SiH](CC)c1ccc(C(C)C)cc1</smiles>

Triethyl(4-isopropylphenyl)silane (23). The reaction was carried out according to the general procedure on $0.2 \mathrm{mmol}$ scale, and $10 \% \mathrm{FeBr}_{3}, 10 \%$ dtbpy was used. The product 23 (25 mg, 54\% yield) was purified with silica gel chromatography (Petroleum ether). ${ }^{1} \mathrm{H}$ NMR (400 MHz, $\left.\mathrm{CDCl}_{3}\right) \delta$ 7.37-7.28 (m, $\left.2 \mathrm{H}\right), 7.33(\mathrm{~d}, J=7.1 \mathrm{~Hz}, 1 \mathrm{H})$, $7.23(\mathrm{~d}, J=7.1 \mathrm{~Hz}, 1 \mathrm{H}), 2.92$ (sept, $J=6.8 \mathrm{~Hz}, 1 \mathrm{H}), 1.28-1.27(\mathrm{~m}, 6 \mathrm{H}), 0.99$ (t, $J=$ $7.8 \mathrm{~Hz}, 9 \mathrm{H}), 0.81$ (q, $J=7.8 \mathrm{~Hz}, 6 \mathrm{H}) .{ }^{13} \mathrm{C} \mathrm{NMR}\left(125 \mathrm{MHz}, \mathrm{CDCl}_{3}\right): \delta$ 147.7, 137.3, 132.4, 131.6, 127.6, 126.7, 34.2, 24.1, 7.5, 3.4. HRMS (EI/TOF): Calculated for $\mathrm{C}_{15} \mathrm{H}_{26} \mathrm{Si}\left(\mathrm{M}^{+}\right)$: 234.1798; Found: 234.1801.<smiles>CCSc1ccc(C(C)(C)c2ccccc2)cc1</smiles>

Triethyl(4-(2-phenylpropan-2-yl)phenyl)silane (24). The reaction was carried out according to the general procedure on $0.2 \mathrm{mmol}$ scale, and $10 \mathrm{~mol} \% \mathrm{Fe}(\mathrm{OTf})_{2}, 10 \mathrm{~mol} \%$ dtbpy were used. The product 24 (37 $\mathrm{mg}, 60 \%$ yield) as a colourless oil was purified with silica gel chromatography (Petroleum ether). ${ }^{1} \mathrm{H}$ NMR $\left(600 \mathrm{MHz}, \mathrm{CDCl}_{3}\right) \delta 7.43$ 7.40 (m, 2 H), 7.33-7.26 (m, 4 H), 7.25-7.22 (m, 2 H), 7.22-7.18 (m, 1 H), 1.72 (s, 6 H), $1.00(\mathrm{t}, J=7.9 \mathrm{~Hz}, 9 \mathrm{H}), 0.80(\mathrm{q}, J=7.9 \mathrm{~Hz}, 6 \mathrm{H}) .{ }^{13} \mathrm{C} \mathrm{NMR}\left(100 \mathrm{MHz}, \mathrm{CDCl}_{3}\right) \delta 151.0$, 150.6, 134.1, 133.9, 127.9, 126.8, 126.0, 125.6, 42.9, 30.6, 7.5, 3.4. HRMS (EI/TOF): Calculated for $\mathrm{C}_{21} \mathrm{H}_{30} \mathrm{Si}\left(\mathrm{M}^{+}\right)$: 310.2111; Found: 310.2116.

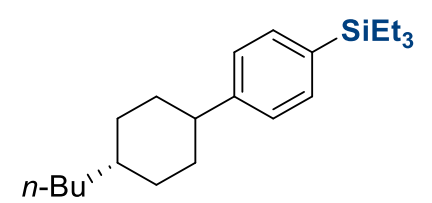

(4-(4-Butylcyclohexyl)phenyl)triethylsilane (25). The reaction was carried out according to the general procedure on $0.2 \mathrm{mmol}$ scale, and $10 \mathrm{~mol} \% \mathrm{FeBr}_{3}, 10 \mathrm{~mol} \%$ dtbpy was used. The product 25 (40 mg, 64\% yield) as a colourless oil was purified with silica gel chromatography (Petroleum ether). ${ }^{1} \mathrm{H} \mathrm{NMR}\left(600 \mathrm{MHz}, \mathrm{CDCl}_{3}\right) \delta 7.46$ $(\mathrm{d}, J=7.8 \mathrm{~Hz}, 2 \mathrm{H}), 7.23(\mathrm{~d}, J=7.8 \mathrm{~Hz}, 2 \mathrm{H}), 2.53-2.45(\mathrm{~m}, 1 \mathrm{H}), 1.97-1.87$ (m, $4 \mathrm{H})$, 
1.57-1.44 (m, 2 H), 1.38-1.21 (m, 7 H), 1.16-1.05 (m, 2 H), 1.02 (t, J = 7.9 Hz, 9 H), 0.97-0.92 (m, $3 \mathrm{H}), 0.83(\mathrm{q}, J=7.9 \mathrm{~Hz}, 6 \mathrm{H}) .{ }^{13} \mathrm{C} \mathrm{NMR}\left(100 \mathrm{MHz}, \mathrm{CDCl}_{3}\right) \delta 148.3$, 134.2, 126.2, 44.6, 37.3, 37.2, 34.2, 33.7, 29.3, 23.1, 14.2, 7.5, 3.5. HRMS (EI/TOF): Calculated for $\mathrm{C}_{22} \mathrm{H}_{38} \mathrm{Si}\left(\mathrm{M}^{+}\right)$: 330.2737; Found: 330.2743 .

$\mathrm{MiEt}_{3}^{\mathrm{Si}_{3}}$

Triethyl(p-tolyl)silane (26). The reaction was carried out according to the general procedure on $0.2 \mathrm{mmol}$ scale, and $10 \% \mathrm{FeBr}_{3}, 10 \%$ dtbpy was used. The product $\mathbf{2 6}$ (28 mg, $68 \%$ yield) was purified with silica gel chromatography (Petroleum ether). ${ }^{1} \mathrm{H}$ NMR (400 MHz, $\left.\mathrm{CDCl}_{3}\right) \delta 7.41(\mathrm{~d}, J=7.7 \mathrm{~Hz}, 2 \mathrm{H}), 7.20(\mathrm{~d}, J=7.7 \mathrm{~Hz}, 2 \mathrm{H}), 2.37$ (s, $3 \mathrm{H}), 0.98(\mathrm{t}, J=7.8 \mathrm{~Hz}, 9 \mathrm{H}), 0.80(\mathrm{q}, J=7.8 \mathrm{~Hz}, 6 \mathrm{H}) .{ }^{13} \mathrm{C} \mathrm{NMR}\left(100 \mathrm{MHz}, \mathrm{CDCl}_{3}\right)$ : $\delta 138.5,134.2,133.7,128.5,21.4,7.4,3.4$. FTMS (EI/Orbitrap): Calculated for $\mathrm{C}_{13} \mathrm{H}_{22} \mathrm{Si}\left(\mathrm{M}^{+}\right): 206.1491 ;$ Found: 206.1484.<smiles>CCCc1ccc(C)cc1</smiles>

Triethyl(4-(methylthio)phenyl)silane (27). This compound is known. ${ }^{11}$ The reaction was carried out according to the general procedure on $0.2 \mathrm{mmol} \mathrm{scale}$, and $10 \mathrm{~mol} \%$ $\mathrm{Fe}(\mathrm{OTf})_{2}, 10 \mathrm{~mol} \%$ dtbpy was used. The product 27 (25 mg, 52\% yield) as a colourless oil was purified with silica gel chromatography (Petroleum ether). ${ }^{1} \mathrm{H}$ NMR $(600 \mathrm{MHz}$, $\left.\mathrm{CDCl}_{3}\right) \delta 7.40(\mathrm{~d}, J=8.1 \mathrm{~Hz}, 2 \mathrm{H}), 7.24(\mathrm{~d}, J=8.1 \mathrm{~Hz}, 2 \mathrm{H}), 2.49(\mathrm{~s}, 3 \mathrm{H}), 0.96(\mathrm{t}, J=$ $7.9 \mathrm{~Hz}, 9 \mathrm{H}), 0.77(\mathrm{q}, J=7.9 \mathrm{~Hz}, 6 \mathrm{H}) .{ }^{13} \mathrm{C} \mathrm{NMR}\left(100 \mathrm{MHz}, \mathrm{CDCl}_{3}\right) \delta 139.2,134.6$, 133.3, 125.5, 15.3, 7.4, 3.3. HRMS (EI/TOF): Calculated for $\mathrm{C}_{13} \mathrm{H}_{22} \mathrm{SSi}\left(\mathrm{M}^{+}\right)$: 283.1211; Found: 238.1209.<smiles>CC[SiH2]c1ccc(Sc2ccc(OC)cc2)cc1</smiles>

4-[(4-Methoxyphenyl)thio]phenylsilane (28). The reaction was carried out according to the general procedure on $0.2 \mathrm{mmol}$ scale, and $10 \mathrm{~mol} \% \mathrm{FeBr}_{3}, 10 \mathrm{~mol} \%$ dtbpy was 
used. The product 28 (33 mg, 50\% yield) as a light yellow oil was purified with silica gel chromatography (Petroleum ether). ${ }^{1} \mathrm{H}$ NMR (400 MHz, $\left.\mathrm{CDCl}_{3}\right) \delta 7.45$ (d, $J=8.5$ $\mathrm{Hz}, 2 \mathrm{H}), 7.34$ (d, J=7.9 Hz, $2 \mathrm{H}), 7.11(\mathrm{~d}, J=7.9 \mathrm{~Hz}, 2 \mathrm{H}), 6.92(\mathrm{~d}, J=8.5 \mathrm{~Hz}, 2 \mathrm{H})$, $3.83(\mathrm{~s}, 3 \mathrm{H}), 0.95(\mathrm{t}, J=7.9 \mathrm{~Hz}, 9 \mathrm{H}), 0.76(\mathrm{q}, J=7.9 \mathrm{~Hz}, 6 \mathrm{H}) .{ }^{13} \mathrm{C} \mathrm{NMR}(100 \mathrm{MHz}$, $\left.\mathrm{CDCl}_{3}\right): \delta 156.0,134.0,135.9,134.7,134.2,126.6,123.5,115.0,55.4,7.4,3.3$.<smiles>CC[SiH](CC)c1ccc2c(c1)OCO2</smiles>

Benzo $[d][1,3]$ dioxol-5-yltriethylsilane (29). This compound is known. ${ }^{9}$ The reaction was carried out according to the general procedure on $0.2 \mathrm{mmol}$ scale, and $10 \mathrm{~mol} \%$ $\mathrm{Fe}(\mathrm{OTf})_{2}, 10 \mathrm{~mol} \%$ dtbpy were used. The product $\mathbf{2 9}(32 \mathrm{mg}, 67 \%$ yield $)$ as a colourless oil was purified with silica gel chromatography (Petroleum ether). ${ }^{1} \mathrm{H}$ NMR (600 MHz, $\left.\mathrm{CDCl}_{3}\right) \delta 6.98(\mathrm{~d}, J=7.6 \mathrm{~Hz}, 1 \mathrm{H}), 6.96(\mathrm{~s}, 1 \mathrm{H}), 6.86(\mathrm{~d}, J=7.6 \mathrm{~Hz}, 1 \mathrm{H}), 5.94(\mathrm{~s}, 2$ $\mathrm{H}), 0.97(\mathrm{t}, J=7.9 \mathrm{~Hz}, 9 \mathrm{H}), 0.77(\mathrm{q}, J=7.9 \mathrm{~Hz}, 6 \mathrm{H}) .{ }^{13} \mathrm{C} \mathrm{NMR}\left(100 \mathrm{MHz}, \mathrm{CDCl}_{3}\right) \delta$ 148.1, 147.3, 130.2, 128.0, 113.4, 108.5, 100.4, 7.4, 3.5. HRMS (EI/TOF): Calculated for $\mathrm{C}_{13} \mathrm{H}_{20} \mathrm{O}_{2} \mathrm{Si}\left(\mathrm{M}^{+}\right)$: 236.1233; Found: 236.1231.

$\mathrm{SiEt}_{3}^{\mathrm{SHO}_{3}}$

Triethyl(4-methoxyphenyl)silane (30). This compound is known. ${ }^{10}$ The reaction was carried out according to the general procedure on $0.2 \mathrm{mmol}$ scale, and $10 \mathrm{~mol} \%$ $\mathrm{Fe}(\mathrm{OTf})_{2}, 10 \mathrm{~mol} \%$ dtbpy was used. The product 30 (28 mg, 63\% yield) as a colourless oil was purified with silica gel chromatography (Petroleum ether). ${ }^{1} \mathrm{H}$ NMR (600 MHz, $\left.\mathrm{CDCl}_{3}\right) \delta 7.44(\mathrm{~d}, J=8.3 \mathrm{~Hz}, 2 \mathrm{H}), 6.93(\mathrm{~d}, J=8.3 \mathrm{~Hz}, 2 \mathrm{H}), 3.83(\mathrm{~s}, 3 \mathrm{H}), 0.98(\mathrm{t}, J=$ $7.9 \mathrm{~Hz}, 9 \mathrm{H}), 0.79$ (q, $J=7.9 \mathrm{~Hz}, 6 \mathrm{H}) .{ }^{13} \mathrm{C} \mathrm{NMR}\left(100 \mathrm{MHz}, \mathrm{CDCl}_{3}\right) \delta 160.1,135.5$, 128.1, 113.4, 54.9, 7.4, 3.5. HRMS (EI/TOF) Calculated for $\mathrm{C}_{13} \mathrm{H}_{22} \mathrm{OSi}\left(\mathrm{M}^{+}\right)$: 222.1440; Found: 222.1435.<smiles>CCCc1ccc(Oc2ccccc2)cc1</smiles> 
Triethyl(4-phenoxyphenyl)silane (31). The reaction was carried out according to the general procedure on $0.2 \mathrm{mmol}$ scale, and $10 \mathrm{~mol} \% \mathrm{Fe}(\mathrm{OTf})_{2}, 10 \mathrm{~mol} \%$ dtbpy was used. The product 31 (40 mg, 70\% yield) as a colourless oil was purified with silica gel chromatography (Petroleum ether). ${ }^{1} \mathrm{H}$ NMR $\left(600 \mathrm{MHz}, \mathrm{CDCl}_{3}\right) \delta 7.47$ (d, J=7.6 Hz, $2 \mathrm{H}), 7.36(\mathrm{t}, J=7.8 \mathrm{~Hz}, 2 \mathrm{H}), 7.13(\mathrm{t}, J=7.4 \mathrm{~Hz}, 1 \mathrm{H}), 7.06(\mathrm{~d}, J=8.1 \mathrm{~Hz}, 2 \mathrm{H}), 7.01$ $(\mathrm{d}, J=7.6 \mathrm{~Hz}, 2 \mathrm{H}), 0.99$ (t, $J=7.9 \mathrm{~Hz}, 9 \mathrm{H}), 0.81$ (q, $J=7.9 \mathrm{~Hz}, 6 \mathrm{H}) .{ }^{13} \mathrm{C} \mathrm{NMR}(100$ $\left.\mathrm{MHz}, \mathrm{CDCl}_{3}\right) \delta 158.1,156.8,135.7,131.3,129.7,123.4,119.3,117.8,7.4,3.4$. HRMS (EI/TOF) Calculated for $\mathrm{C}_{18} \mathrm{H}_{24} \mathrm{OSi}\left(\mathrm{M}^{+}\right)$: 284.1591; Found: 284.1585 .<smiles>CCCC1=CCCc2ccccc21</smiles>

(3,4-Dihydronaphthalen-1-yl)triethylsilane (32). The product $(41.5 \mathrm{mg}, 85 \%$ yield) as a light yellow liquid was purified with silica gel chromatography (Petroleum ether). ${ }^{1} \mathrm{H}$ NMR $\left(400 \mathrm{MHz}, \mathrm{CDCl}_{3}\right) \delta 7.26(\mathrm{~d}, J=6.8 \mathrm{~Hz}, 1 \mathrm{H}), 7.19-7.10(\mathrm{~m}, 3 \mathrm{H}), 6.44(\mathrm{t}, J$ $=4.4 \mathrm{~Hz}, 1 \mathrm{H}), 2.71(\mathrm{t}, J=8.0 \mathrm{~Hz}, 2 \mathrm{H}), 2.27(\mathrm{dt}, J=4.4,8.0 \mathrm{~Hz}, 2 \mathrm{H}), 0.96(\mathrm{t}, J=8.0$ $\mathrm{Hz}, 9 \mathrm{H}), 0.79$ (q, $J=8.0 \mathrm{~Hz}, 6 \mathrm{H}) .{ }^{13} \mathrm{C} \mathrm{NMR}\left(100 \mathrm{MHz}, \mathrm{CDCl}_{3}\right) \delta 141.2,137.0,136.1$, 134.7, 127.7, 126.2, 126.1, 126.0, 28.2, 24.3, 7.5, 3.9. HRMS (EI/TOF): Calculated for $\mathrm{C}_{16} \mathrm{H}_{24} \mathrm{Si}\left(\mathrm{M}^{+}\right):$244.1647; Found: 244.1649 .<smiles>CCCC1=CCC(C)c2ccccc21</smiles>

Triethyl(4-methyl-3,4-dihydronaphthalen-1-yl)silane (33). The product (36.1 mg, $70 \%$ yield) as a light yellow liquid was purified with silica gel chromatography (Petroleum ether). ${ }^{1} \mathrm{H}$ NMR (400 MHz, $\left.\mathrm{CDCl}_{3}\right) \delta$ 7.28-7.25 (m, $\left.1 \mathrm{H}\right)$, 7.19-7.14 (m, 3 H), 6.34 (t, $J=4.4 \mathrm{~Hz}, 1 \mathrm{H}), 2.87-2.82(\mathrm{~m}, 1 \mathrm{H}), 2.44$ (ddd, $J=16.0,6.4,4.0 \mathrm{~Hz}, 1 \mathrm{H}$ ), $2.12(\mathrm{ddd}, J=16.8,6.8,5.2 \mathrm{~Hz}, 1 \mathrm{H}), 1.20(\mathrm{~d}, J=6.8 \mathrm{~Hz}, 3 \mathrm{H}), 0.94(\mathrm{t}, J=8.0 \mathrm{~Hz}, 9$ $\mathrm{H}), 0.78(\mathrm{q}, J=8.0 \mathrm{~Hz}, 6 \mathrm{H}) .{ }^{13} \mathrm{C} \mathrm{NMR}\left(100 \mathrm{MHz}, \mathrm{CDCl}_{3}\right) \delta 140.9,139.7,136.2,134.2$, 
126.5, 126.3, 126.0, 126.0, 32.2, 32.0, 19.6, 7.5, 3.9. HRMS (EI/TOF): Calculated for $\mathrm{C}_{17} \mathrm{H}_{26} \mathrm{Si}\left(\mathrm{M}^{+}\right)$: 258.1804; Found: 258.1807.<smiles>CCCC1=CCCc2c(OCc3ccccc3)cccc21</smiles>

(5-(benzyloxy)-3,4-dihydronaphthalen-1-yl)triethylsilane (34). The product (44.1 mg, 63\% yield) as a light yellow liquid was purified with silica gel chromatography (Petroleum ether). ${ }^{1} \mathrm{H}$ NMR (400 MHz, $\left.\mathrm{CDCl}_{3}\right) \delta 7.47(\mathrm{~d}, J=7.6 \mathrm{~Hz}, 2 \mathrm{H}), 7.42-7.38$ (m, $2 \mathrm{H}), 7.35-7.31(\mathrm{~m}, 1 \mathrm{H}), 7.12(\mathrm{t}, J=8.0 \mathrm{~Hz}, 1 \mathrm{H}), 6.95(\mathrm{~d}, J=8.0 \mathrm{~Hz}, 1 \mathrm{H}), 6.84$ $(\mathrm{d}, J=8.4 \mathrm{~Hz}, 1 \mathrm{H}), 6.46(\mathrm{t}, J=4.4 \mathrm{~Hz}, 1 \mathrm{H}), 5.09$ (s, $2 \mathrm{H}), 2.79$ (t, $J=8.0 \mathrm{~Hz}, 2 \mathrm{H})$, $2.24(\mathrm{dt}, J=4.4,8.0 \mathrm{~Hz}, 2 \mathrm{H}), 0.95(\mathrm{t}, J=8.0 \mathrm{~Hz}, 9 \mathrm{H}), 0.78(\mathrm{q}, J=8.0 \mathrm{~Hz}, 6 \mathrm{H}) .{ }^{13} \mathrm{C}$ NMR $\left(100 \mathrm{MHz} \mathrm{CDCl}_{3}\right) \delta 155.3,141.6,138.2,137.6,134.5,128.5,127.7,127.2,126.1$, 124.4, 119.5, 110.6, 70.2, 23.7, 19.8, 7.5, 3.9. HRMS (EI/TOF): Calculated for $\mathrm{C}_{23} \mathrm{H}_{31} \mathrm{OSi}(\mathrm{M}+\mathrm{H})^{+}:$351.2139; Found: 351.2130.<smiles>CCCC1=CCCc2ccc(C)cc21</smiles>

Triethyl(7-methyl-3,4-dihydronaphthalen-1-yl)silane (35). The product (37.2 mg, $72 \%$ yield) as a light yellow liquid was purified with silica gel chromatography (Petroleum ether). ${ }^{1} \mathrm{H}$ NMR (400 MHz, $\left.\mathrm{CDCl}_{3}\right) \delta 7.06$ (s, $\left.1 \mathrm{H}\right), 7.02(\mathrm{~d}, J=7.6 \mathrm{~Hz}, 1$ H), $6.93(\mathrm{~d}, J=7.2 \mathrm{~Hz}, 1 \mathrm{H}), 6.42(\mathrm{t}, J=4.4 \mathrm{~Hz}, 1 \mathrm{H}), 2.65(\mathrm{t}, J=8.0 \mathrm{~Hz}, 2 \mathrm{H}), 2.32(\mathrm{~s}$, $3 \mathrm{H}), 2.24(\mathrm{dt}, J=4.4,8.0 \mathrm{~Hz}, 2 \mathrm{H}), 0.95(\mathrm{t}, J=8.0 \mathrm{~Hz}, 9 \mathrm{H}), 0.78(\mathrm{q}, J=8.0 \mathrm{~Hz}, 6 \mathrm{H})$. ${ }^{13} \mathrm{C} \mathrm{NMR}\left(100 \mathrm{MHz}, \mathrm{CDCl}_{3}\right) \delta 141.2,136.9,135.4,134.7,133.0,127.5,126.9,126.7$, 27.7, 24.5, 21.4, 7.5, 3.9. HRMS (EI/TOF): Calculated for $\mathrm{C}_{17} \mathrm{H}_{26} \mathrm{Si}\left(\mathrm{M}^{+}\right)$: 258.1804; Found: 258.1801. 


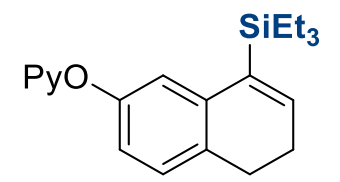

2-((8-(Triethylsilyl)-5,6-dihydronaphthalen-2-yl)oxy)pyridine (36). The product (48.6 mg, 72\% yield) as a light yellow liquid was purified with silica gel chromatography (Petroleum ether). ${ }^{1} \mathrm{H}$ NMR (400 MHz, $\left.\mathrm{CDCl}_{3}\right) \delta 8.21$ (d, $J=4.8 \mathrm{~Hz}$, $1 \mathrm{H}), 7.68-7.63(\mathrm{~m}, 1 \mathrm{H}), 7.12(\mathrm{~d}, J=4.0 \mathrm{~Hz}, 1 \mathrm{H}), 7.00(\mathrm{~d}, J=2.0 \mathrm{~Hz}, 1 \mathrm{H}), 6.98-6.95$ (m, $1 \mathrm{H}), 6.89-6.86(\mathrm{~m}, 2 \mathrm{H}), 6.44(\mathrm{t}, J=4.4 \mathrm{~Hz}, 1 \mathrm{H}), 2.68(\mathrm{t}, J=8.0 \mathrm{~Hz}, 2 \mathrm{H}), 2.26$ $(\mathrm{dt}, J=4.4,8.0 \mathrm{~Hz}, 2 \mathrm{H}), 0.89(\mathrm{t}, J=8.0 \mathrm{~Hz}, 9 \mathrm{H}), 0.70(\mathrm{q}, J=8.0 \mathrm{~Hz}, 6 \mathrm{H}) .{ }^{13} \mathrm{C} \mathrm{NMR}$ $\left(100 \mathrm{MHz}, \mathrm{CDCl}_{3}\right) \delta 163.9,152.4,147.8,141.7,139.2,138.2,134.5,132.2,128.4$, 128.1, 118.8, 118.2, 111.2, 27.5, 24.4, 7.4, 3.7. HRMS (EI/TOF): Calculated for $\mathrm{C}_{17} \mathrm{H}_{27} \mathrm{ONSi}\left(\mathrm{M}^{+}\right)$: 337.1856; Found: 337.1868.

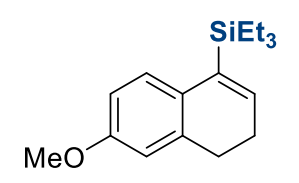

Triethyl(6-methoxy-3,4-dihydronaphthalen-1-yl)silane (37). The product (39.4 mg, $72 \%$ yield) as a light yellow solid was purified with silica gel chromatography (Petroleum ether). ${ }^{1} \mathrm{H}$ NMR (400 MHz, $\left.\mathrm{CDCl}_{3}\right) \delta$ 7.18-7.16 (m, $\left.1 \mathrm{H}\right)$, 6.70-6.69 (m, 2 H), $6.30(\mathrm{t}, J=4.4 \mathrm{~Hz}, 1 \mathrm{H}), 3.80(\mathrm{~s}, 3 \mathrm{H}), 2.67(\mathrm{t}, J=8.0 \mathrm{~Hz}, 2 \mathrm{H}), 2.26-2.21(\mathrm{~m}, 2 \mathrm{H})$, $0.93(\mathrm{t}, J=8.0 \mathrm{~Hz}, 9 \mathrm{H}), 0.76(\mathrm{q}, J=8.0 \mathrm{~Hz}, 6 \mathrm{H}) .{ }^{13} \mathrm{C} \mathrm{NMR}\left(100 \mathrm{MHz}, \mathrm{CDCl}_{3}\right) \delta 157.8$, 138.7, 137.9, 134.0, 130.2, 126.9, 113.9, 110.7, 55.1, 28.7, 24.1, 7.5, 3.9. HRMS (EI/TOF): Calculated for $\mathrm{C}_{17} \mathrm{H}_{27} \mathrm{OSi}(\mathrm{M}+\mathrm{H})^{+}:$: 275.1826; Found: 275.1824 .

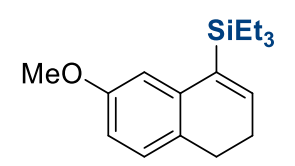

Triethyl(7-methoxy-3,4-dihydronaphthalen-1-yl)silane (38). The product (38.3 mg, $70 \%$ yield) as a light yellow liquid was purified with silica gel chromatography (Petroleum ether). ${ }^{1} \mathrm{H}$ NMR (400 MHz, $\left.\mathrm{CDCl}_{3}\right) \delta$ 7.18-7.15 (m, $\left.1 \mathrm{H}\right), 6.71-6.68$ (m, 2 H), $6.30(\mathrm{t}, J=4.4 \mathrm{~Hz}, 1 \mathrm{H}), 3.80(\mathrm{~s}, 3 \mathrm{H}), 2.68(\mathrm{t}, J=8.0 \mathrm{~Hz}, 2 \mathrm{H}), 2.26-2.21(\mathrm{~m}, 2 \mathrm{H})$, $0.94(\mathrm{t}, J=8.0 \mathrm{~Hz}, 9 \mathrm{H}), 0.76(\mathrm{q}, J=8.0 \mathrm{~Hz}, 6 \mathrm{H}) .{ }^{13} \mathrm{C} \mathrm{NMR}\left(100 \mathrm{MHz}, \mathrm{CDCl}_{3}\right) \delta 157.8$, 
$138.7,137.9,134.0,130.2,126.9,113.9,110.7,55.1,28.7,24.2,7.5,3.9$. HRMS (EI/TOF): Calculated for $\mathrm{C}_{17} \mathrm{H}_{26} \mathrm{OSi}\left(\mathrm{M}^{+}\right)$: 274.1753; Found: 274.1761 .<smiles>CCCC1=CCCc2cc(N(C)OC)ccc21</smiles>

$N, N$-Dimethyl-5-(triethylsilyl)-7,8-dihydronaphthalen-2-amine (39). The product (35.6 mg, 62\% yield) as a light yellow oil was purified with silica gel chromatography (Petroleum ether/ EA = 20/1). ${ }^{1} \mathrm{H}$ NMR $\left(400 \mathrm{MHz}, \mathrm{CDCl}_{3}\right) \delta$ 7.16-7.13 (m, $\left.1 \mathrm{H}\right), 6.55-$ $6.54(\mathrm{~m}, 2 \mathrm{H}), 6.21(\mathrm{t}, J=4.4 \mathrm{~Hz}, 1 \mathrm{H}), 2.95(\mathrm{~s}, 6 \mathrm{H}), 2.68(\mathrm{t}, J=8.0 \mathrm{~Hz}, 2 \mathrm{H}), 2.27-$ $2.22(\mathrm{~m}, 2 \mathrm{H}), 0.94(\mathrm{t}, J=8.0 \mathrm{~Hz}, 9 \mathrm{H}), 0.76(\mathrm{q}, J=8.0 \mathrm{~Hz}, 6 \mathrm{H}) .{ }^{13} \mathrm{C}$ NMR (100 MHz, $\left.\mathrm{CDCl}_{3}\right) \delta 148.9,137.2,137.0,134.0,126.9,112.5,109.9,40.6,29.2,24.5,7.6,3.9$. FTMS (ESI/Quadrupole): Calculated for $\mathrm{C}_{18} \mathrm{H}_{30} \mathrm{NSi}(\mathrm{M}+\mathrm{H})^{+}$: 288.2142; Found: 288.2144.<smiles>CC[Si](C)(C=Cc1ccccc1)Cc1ccccc1</smiles>

(1,3-Diphenylprop-1-en-2-yl)triethylsilane (40). The product (33.9 mg, 55\% yield) as a light yellow oil was purified with silica gel chromatography (Petroleum ether). ${ }^{1} \mathrm{H}$ NMR (400 MHz, $\left.\mathrm{CDCl}_{3}\right) \delta$ 7.31-7.20 (m, $\left.10 \mathrm{H}\right), 7.02$ (s, $\left.1 \mathrm{H}\right), 3.77$ (s, $\left.2 \mathrm{H}\right), 0.89$ (t, $J$ $=8.0 \mathrm{~Hz}, 9 \mathrm{H}), 0.52(\mathrm{q}, J=8.0 \mathrm{~Hz}, 6 \mathrm{H}) .{ }^{13} \mathrm{C} \mathrm{NMR}\left(100 \mathrm{MHz}, \mathrm{CDCl}_{3}\right) \delta 140.8,140.3$, $139.2,137.9,128.7,128.4,128.24,128.17,126.8,125.9,36.5,7.3,3.1$. HRMS (EI/TOF): Calculated for $\mathrm{C}_{21} \mathrm{H}_{28} \mathrm{Si}\left(\mathrm{M}^{+}\right)$: 308.1960; Found: 309.1969 .<smiles>CC[SiH2]C1=Cc2ccccc2CC1</smiles>

(3,4-Dihydronaphthalen-2-yl)triethylsilane (41). The product (26.8 mg, $55 \%$ yield) as light yellow liquid was purified with silica gel chromatography (Petroleum ether). ${ }^{1} \mathrm{H}$ NMR (400 MHz, $\left.\mathrm{CDCl}_{3}\right) \delta$ 7.26-7.10 (m, $\left.3 \mathrm{H}\right), 7.05(\mathrm{~d}, J=7.2 \mathrm{~Hz}, 1 \mathrm{H}), 6.71(\mathrm{~s}, 1$ H), $2.73(\mathrm{t}, J=8.0 \mathrm{~Hz}, 2 \mathrm{H}), 2.28(\mathrm{t}, J=8.0 \mathrm{~Hz}, 2 \mathrm{H}), 0.98(\mathrm{t}, J=8.0 \mathrm{~Hz}, 9 \mathrm{H}), 0.69(\mathrm{q}$, 
$J=8.0 \mathrm{~Hz}, 6 \mathrm{H}) .{ }^{13} \mathrm{C} \mathrm{NMR}\left(100 \mathrm{MHz}, \mathrm{CDCl}_{3}\right) \delta 139.0,136.4,136.1,134.1,127.4$, 127.0, 126.4 125.9, 27.4, 25.8, 7.5, 2.5. HRMS (EI/TOF): Calculated for $\mathrm{C}_{16} \mathrm{H}_{24} \mathrm{Si}\left(\mathrm{M}^{+}\right)$: 244.1647; Found: 244.1645.<smiles>CCC1=Cc2cc(OC)ccc2CC1</smiles>

Triethyl(7-methoxy-3,4-dihydronaphthalen-2-yl)silane (42). The product (27.4 mg, $50 \%$ yield) as light yellow liquid was purified with silica gel chromatography (Petroleum ether). ${ }^{1} \mathrm{H}$ NMR (400 MHz, $\left.\mathrm{CDCl}_{3}\right) \delta 7.00$ (d, J=8.0 Hz, $\left.1 \mathrm{H}\right), 6.69-6.63$ $(\mathrm{m}, 3 \mathrm{H}), 3.79(\mathrm{~s}, 3 \mathrm{H}), 2.64(\mathrm{t}, J=8.0 \mathrm{~Hz}, 2 \mathrm{H}), 2.25(\mathrm{t}, J=8.0 \mathrm{~Hz}, 2 \mathrm{H}), 0.96(\mathrm{t}, J=$ $8.0 \mathrm{~Hz}, 9 \mathrm{H}), 0.67(\mathrm{q}, J=8.0 \mathrm{~Hz}, 6 \mathrm{H}) .{ }^{13} \mathrm{C} \mathrm{NMR}\left(100 \mathrm{MHz}, \mathrm{CDCl}_{3}\right) \delta 158.3,139.8$, 136.4, 135.0, 128.3, 128.0, 112.4, 111.3, 55.3, 26.6, 26.3, 7.5, 2.5. HRMS (EI/TOF): Calculated for $\mathrm{C}_{17} \mathrm{H}_{26} \mathrm{OSi}\left(\mathrm{M}^{+}\right)$: 274.1747; Found: 274.1754 .

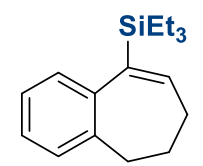

(6,7-Dihydro-5 $\boldsymbol{H}$-benzo[7]annulen-9-yl)triethylsilane (43). The product (36.1 mg, $70 \%$ yield) as light yellow oil was purified with silica gel chromatography (Petroleum ether). ${ }^{1} \mathrm{H}$ NMR (400 MHz, $\left.\mathrm{CDCl}_{3}\right) \delta$ 7.20-7.16 (m, $\left.2 \mathrm{H}\right), 7.12-7.09$ (m, $\left.1 \mathrm{H}\right), 7.05$ (d, $J=7.6 \mathrm{~Hz}, 1 \mathrm{H}), 6.48(\mathrm{t}, J=7.6 \mathrm{~Hz}, 1 \mathrm{H}), 2.50(\mathrm{t}, J=7.2 \mathrm{~Hz}, 2 \mathrm{H}), 2.11-2.04(\mathrm{~m}, 2 \mathrm{H})$, $1.90(\mathrm{q}, J=7.2 \mathrm{~Hz}, 2 \mathrm{H}), 0.92(\mathrm{t}, J=8.0 \mathrm{~Hz}, 9 \mathrm{H}), 0.66(\mathrm{q}, J=8.0 \mathrm{~Hz}, 6 \mathrm{H}) .{ }^{13} \mathrm{C} \mathrm{NMR}$ $\left(100 \mathrm{MHz} \mathrm{CDCl}_{3}\right) \delta 142.4,142.1,141.5,140.2$, 128.6, 127.3, 125.6, 34.5, 32.0, 26.1, 7.4, 3.8. HRMS (EI/TOF): Calculated for $\mathrm{C}_{17} \mathrm{H}_{26} \mathrm{Si}\left(\mathrm{M}^{+}\right)$: 294.1804; Found: 294.1815.<smiles>CC[SiH2]C1=CCCc2ccc(-c3cccc(OC)c3)cc21</smiles>

Triethyl(7-(3-methoxyphenyl)-3,4-dihydronaphthalen-1-yl)silane (44). The product (57.4 mg, 82\% yield) as a colourless oil was purified with silica gel chromatography (Petroleum ether). ${ }^{1} \mathrm{H}$ NMR (400 MHz, $\left.\mathrm{CDCl}_{3}\right) \delta 7.50$ (s, $\left.1 \mathrm{H}\right), 7.39-7.34$ (m, $\left.2 \mathrm{H}\right)$, 7.21-7.16 (m, 2 H), $7.12(\mathrm{~s}, 1 \mathrm{H}), 6.90(\mathrm{dd}, J=8.0,1.6 \mathrm{~Hz}, 1 \mathrm{H}), 6.49$ (t, $J=4.4 \mathrm{~Hz}, 1$ 
H), $3.88(\mathrm{~s}, 3 \mathrm{H}), 2.75$ (t, $J=8.0 \mathrm{~Hz}, 2 \mathrm{H}), 2.34-2.28(\mathrm{~m}, 2 \mathrm{H}), 0.98(\mathrm{t}, J=8.0 \mathrm{~Hz}, 9 \mathrm{H})$, $0.82(\mathrm{q}, J=8.0 \mathrm{~Hz}, 6 \mathrm{H}) .{ }^{13} \mathrm{C} \mathrm{NMR}\left(100 \mathrm{MHz}, \mathrm{CDCl}_{3}\right) \delta 159.9,142.1,141.6,139.0$, 137.4, 135.4, 134.7, 129.7, 128.0, 124.94, 124.86, 119.4, 112.5, 55.2, 27.8, 24.4, 7.6, 4.0. HRMS (EI/TOF): Calculated for $\mathrm{C}_{23} \mathrm{H}_{30} \mathrm{OSi}\left(\mathrm{M}^{+}\right)$: 351.2139; Found: 351.2130.<smiles>CC[SiH2]C1=CCCc2ccc(-c3ccc(C(F)(F)F)cc3)cc21</smiles>

Triethyl(7-(4-(trifluoromethyl)phenyl)-3,4-dihydronaphthalen-1-yl)silane

(45).

The product (58.2 mg, 75\% yield, m.p. 39.6-42.1 ${ }^{\circ} \mathrm{C}$ ) as a light yellow solid was purified with silica gel chromatography (Petroleum ether). ${ }^{1} \mathrm{H} \mathrm{NMR}\left(400 \mathrm{MHz}, \mathrm{CDCl}_{3}\right) \delta 7.71$ $(\mathrm{d}, J=8.4 \mathrm{~Hz}, 2 \mathrm{H}), 7.66(\mathrm{~d}, J=8.4 \mathrm{~Hz}, 2 \mathrm{H}), 7.49$ (s, $1 \mathrm{H}), 7.36(\mathrm{dd}, J=7.6,1.6 \mathrm{~Hz}$, $1 \mathrm{H}), 7.23(\mathrm{~d}, J=8.0 \mathrm{~Hz}, 1 \mathrm{H}), 6.51(\mathrm{t}, J=4.4 \mathrm{~Hz}, 1 \mathrm{H}), 2.76(\mathrm{t}, J=8.0 \mathrm{~Hz}, 2 \mathrm{H}), 2.32$ $(\mathrm{dt}, J=4.4,8.0 \mathrm{~Hz}, 2 \mathrm{H}), 0.98(\mathrm{t}, J=7.6 \mathrm{~Hz}, 9 \mathrm{H}), 0.82(\mathrm{q}, J=7.6 \mathrm{~Hz}, 6 \mathrm{H}) .{ }^{13} \mathrm{C} \mathrm{NMR}$ $\left(100 \mathrm{MHz}, \mathrm{CDCl}_{3}\right) \delta 145.1,142.0,137.70,137.67,136.3,134.5,128.9\left(\mathrm{q},{ }^{2} J_{\mathrm{CF}}=32.0\right.$ $\mathrm{Hz}), 128.3,125.7\left(\mathrm{q},{ }^{3} J_{\mathrm{CF}}=4.0 \mathrm{~Hz}\right), 124.94,124.90,27.8,24.3,7.5,4.0$. HRMS (EI/TOF): Calculated for $\mathrm{C}_{23} \mathrm{H}_{27} \mathrm{~F}_{3} \mathrm{Si}\left(\mathrm{M}^{+}\right)$: 388.1833; Found: 388.1834 .<smiles>CC[SiH2]C1=CCCc2ccc(Cl)cc21</smiles>

(7-Chloro-3,4-dihydronaphthalen-1-yl)triethylsilane (46). The product (40.0 mg, $72 \%$ yield) as a light yellow oil was purified with silica gel chromatography (Petroleum ether). ${ }^{1} \mathrm{H}$ NMR $\left(400 \mathrm{MHz}, \mathrm{CDCl}_{3}\right) \delta 7.20(\mathrm{~s}, 1 \mathrm{H}), 7.08-7.03(\mathrm{~m}, 2 \mathrm{H}), 6.47(\mathrm{t}, J=4.4$ $\mathrm{Hz}, 1 \mathrm{H}), 2.65$ (t, $J=8.0 \mathrm{~Hz}, 2 \mathrm{H}), 2.27-2.22(\mathrm{~m}, 2 \mathrm{H}), 0.94(\mathrm{t}, J=7.6 \mathrm{~Hz}, 9 \mathrm{H}), 0.78$ $(\mathrm{q}, J=7.6 \mathrm{~Hz}, 6 \mathrm{H}) .{ }^{13} \mathrm{C} \mathrm{NMR}\left(100 \mathrm{MHz}, \mathrm{CDCl}_{3}\right) \delta 142.4,138.6,134.4,134.1,131.6$, 128.7, 125.9, 125.8, 27.5, 24.2, 7.5, 3.8. HRMS (EI/TOF): Calculated for $\mathrm{C}_{16} \mathrm{H}_{23} \mathrm{SiCl}$ $\left(\mathrm{M}^{+}\right)$: 278.1254; Found: 278.1258 .<smiles>CCCC1=CCCc2ccc(F)cc21</smiles> 
Triethyl(7-fluoro-3,4-dihydronaphthalen-1-yl)silane (47). The product (36.7 mg, $70 \%$ yield) as a colourless oil was purified with silica gel chromatography (Petroleum ether). ${ }^{1} \mathrm{H}$ NMR $\left(400 \mathrm{MHz}, \mathrm{CDCl}_{3}\right) \delta 7.06(\mathrm{t}, J=7.6 \mathrm{~Hz}, 1 \mathrm{H}), 6.95(\mathrm{dd}, J=10.4,2.4 \mathrm{~Hz}, 1$ H), $6.80(\mathrm{td}, J=8.4,2.4 \mathrm{~Hz}, 1 \mathrm{H}), 6.48(\mathrm{t}, J=4.4 \mathrm{~Hz}, 1 \mathrm{H}), 2.65(\mathrm{t}, J=8.0 \mathrm{~Hz}, 2 \mathrm{H})$, $2.25(\mathrm{dt}, J=4.4,8.0 \mathrm{~Hz}, 2 \mathrm{H}), 0.94(\mathrm{t}, J=8.0 \mathrm{~Hz}, 9 \mathrm{H}), 0.78(\mathrm{q}, J=8.0 \mathrm{~Hz}, 6 \mathrm{H}) .{ }^{13} \mathrm{C}$ $\operatorname{NMR}\left(100 \mathrm{MHz}, \mathrm{CDCl}_{3}\right) \delta 161.5\left(\mathrm{~d},{ }^{1} J_{\mathrm{CF}}=240.0 \mathrm{~Hz}\right), 142.4,138.6\left(\mathrm{~d},{ }^{3} J_{\mathrm{CF}}=8.0 \mathrm{~Hz}\right)$, 134.2, $131.4\left(\mathrm{~d},{ }^{4} J_{\mathrm{CF}}=3.0 \mathrm{~Hz}\right), 133.4\left(\mathrm{~d},{ }^{3} J_{\mathrm{CF}}=8.0 \mathrm{~Hz}\right), 112.7\left(\mathrm{~d},{ }^{2} J_{\mathrm{CF}}=21.0 \mathrm{~Hz}\right), 112.2$ $\left(\mathrm{d},{ }^{2} J_{\mathrm{CF}}=21.0 \mathrm{~Hz}\right), 27.3,24.4,7.4,3.8$. HRMS (EI/TOF): Calculated for $\mathrm{C}_{16} \mathrm{H}_{23} \mathrm{FSi}$ $\left(\mathrm{M}^{+}\right)$: 262.1552; Found: 262.1553.

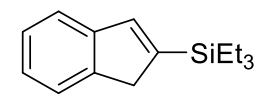

Triethyl(1H-inden-2-yl)silane (48). The product (24.4 mg, 53\% yield) as a colourless oil was purified with silica gel chromatography (Petroleum ether). ${ }^{1} \mathrm{H}$ NMR (400 MHz, $\left.\mathrm{CDCl}_{3}\right) \delta 7.48(\mathrm{~d}, J=7.2 \mathrm{~Hz}, 1 \mathrm{H}), 7.40(\mathrm{~d}, J=7.6 \mathrm{~Hz}, 1 \mathrm{H}), 7.28-7.25(\mathrm{~m}, 1 \mathrm{H})$, 7.19$7.15(\mathrm{~m}, 1 \mathrm{H}), 7.12(\mathrm{~s}, 1 \mathrm{H}), 3.44(\mathrm{~s}, 2 \mathrm{H}), 0.98(\mathrm{t}, J=8.0 \mathrm{~Hz}, 9 \mathrm{H}), 0.73$ (q, $J=8.0 \mathrm{~Hz}$, $6 \mathrm{H}) .{ }^{13} \mathrm{C} \mathrm{NMR}\left(100 \mathrm{MHz}, \mathrm{CDCl}_{3}\right) \delta 146.7,146.4,145.6,141.6,126.2,124.5,123.5$, 120.6, 43.1, 7.5, 3.6. HRMS (EI/TOF): Calculated for $\mathrm{C}_{15} \mathrm{H}_{22} \mathrm{Si}\left(\mathrm{M}^{+}\right)$: 230.1491; Found: 230.1490 .<smiles>CC[SiH2]C1=CCC(c2ccccc2)CC1</smiles>

Triethyl(1,2,3,6-tetrahydro-[1,1'-biphenyl]-4-yl)silane (49). The product (32.6 mg, $60 \%$ yield) as a colourless oil was purified with silica gel chromatography (Petroleum ether). ${ }^{1} \mathrm{H}$ NMR (400 MHz, $\left.\mathrm{CDCl}_{3}\right) \delta$ 7.33-7.29 (m, $\left.2 \mathrm{H}\right)$, 7.24-7.18 (m, $\left.3 \mathrm{H}\right)$, 6.08-6.06 (m, $1 \mathrm{H}), 2.82-2.77(\mathrm{~m}, 1 \mathrm{H}), 2.41-2.36(\mathrm{~m}, 1 \mathrm{H}), 2.25-2.20$ (m, $3 \mathrm{H}), 1.97-1.94(\mathrm{~m}, 1$ $\mathrm{H}), 1.77-1.69(\mathrm{~m}, 1 \mathrm{H}), 0.96(\mathrm{t}, J=8.0 \mathrm{~Hz}, 9 \mathrm{H}), 0.61(\mathrm{q}, J=8.0 \mathrm{~Hz}, 6 \mathrm{H}) .{ }^{13} \mathrm{C} \mathrm{NMR}$ $\left(100 \mathrm{MHz}, \mathrm{CDCl}_{3}\right) \delta 147.5,136.5,135.5,128.3,126.9,125.9,40.1,35.1,30.1,28.2$, 7.5, 2.6. HRMS (EI/TOF): Calculated for $\mathrm{C}_{18} \mathrm{H}_{28} \mathrm{Si}\left(\mathrm{M}^{+}\right)$: 272.1960; Found: 272.1959. 
$\underbrace{\mathrm{SiEt}}_{\mathrm{OBn}}$

(4-(Benzyloxy)cyclohex-1-en-1-yl)triethylsilane (50). The product $(30.2 \mathrm{mg}, 50 \%$ yield) as a colourless oil was purified with silica gel chromatography (Petroleum ether). ${ }^{1} \mathrm{H}$ NMR (400 MHz, $\left.\mathrm{CDCl}_{3}\right) \delta$ 7.36-7.31 (m, $\left.4 \mathrm{H}\right)$, 7.27-7.25 (m, $\left.1 \mathrm{H}\right)$, 5.85-5.84 (m, 1 H), $4.60(\mathrm{~d}, J=12.0 \mathrm{~Hz}, 1 \mathrm{H}), 4.56(\mathrm{~d}, J=12.0 \mathrm{~Hz}, 1 \mathrm{H}), 3.66-3.59(\mathrm{~m}, 1 \mathrm{H}), 2.49-2.45$ (m, $1 \mathrm{H}), 2.25-2.19(\mathrm{~m}, 1 \mathrm{H}), 2.15-1.98(\mathrm{~m}, 4 \mathrm{H}), 0.90(\mathrm{t}, J=8.0 \mathrm{~Hz}, 9 \mathrm{H}), 0.55(\mathrm{q}, J=$ $8.0 \mathrm{~Hz}, 6 \mathrm{H}) .{ }^{13} \mathrm{C}$ NMR $\left(100 \mathrm{MHz}, \mathrm{CDCl}_{3}\right) \delta 139.1,135.7,134.1,128.3,127.5,127.3$, 74.1, 69.7, 33.6, 28.5, 26.8, 7.4, 2.5. FTMS (ESI/Quadrupole): Calculated for $\mathrm{C}_{19} \mathrm{H}_{30} \mathrm{NaOSi}(\mathrm{M}+\mathrm{Na})^{+}:$325.1958; Found: 325.1964.

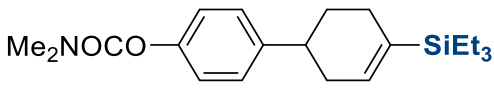

4'-(Triethylsilyl)-1',2',3',6'-tetrahydro-[1,1'-biphenyl]-4-yl-dimethylcarbamate

(51). The product $(23.0 \mathrm{mg}, 32 \%$ yield) as a colourless oil was purified with silica gel chromatography (Petroleum ether/EA = 20/1). ${ }^{1} \mathrm{H}$ NMR $\left(400 \mathrm{MHz}, \mathrm{CDCl}_{3}\right) \delta 7.19(\mathrm{~d}$, $J=8.4 \mathrm{~Hz}, 2 \mathrm{H}), 7.03(\mathrm{~d}, J=8.4 \mathrm{~Hz}, 2 \mathrm{H}), 6.05-6.04(\mathrm{~m}, 1 \mathrm{H}), 3.09(\mathrm{~s}, 3 \mathrm{H}), 3.00(\mathrm{~s}, 3$ H), 2.80-2.75 (m, $1 \mathrm{H}), 2.38-2.33(\mathrm{~m}, 1 \mathrm{H}), 2.19-2.13(\mathrm{~m}, 3 \mathrm{H}), 1.94-1.91(\mathrm{~m}, 1 \mathrm{H})$, 1.72-1.65 (m, $1 \mathrm{H}), 0.94(\mathrm{t}, J=8.0 \mathrm{~Hz}, 9 \mathrm{H}), 0.59$ (q, $J=8.0 \mathrm{~Hz}, 6 \mathrm{H}) .{ }^{13} \mathrm{C}$ NMR $(100$ $\left.\mathrm{MHz}, \mathrm{CDCl}_{3}\right) \delta 155.1,149.6,144.4,136.4,135.5,127.6,121.4,39.5,36.7,36.4,35.2$, 30.2, 28.2. FTMS (ESI/Quadrupole): Calculated for $\mathrm{C}_{21} \mathrm{H}_{33} \mathrm{NNaO}_{2} \mathrm{Si}(\mathrm{M}+\mathrm{Na})^{+}$: 382.2173; Found: 382.2178.

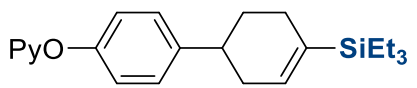

2-((4'-(Triethylsilyl)-1',2',3',6'-tetrahydro-[1,1'-biphenyl]-4-yl)oxy)pyridine (52). The product (30.6 mg, $42 \%$ yield, m.p. $58.8-62.1^{\circ} \mathrm{C}$ ) as a white solid was purified with silica gel chromatography (Petroleum ether/ EA = 20/1). ${ }^{1} \mathrm{H}$ NMR $\left(400 \mathrm{MHz}, \mathrm{CDCl}_{3}\right)$ $\delta 8.20(\mathrm{~d}, J=3.6 \mathrm{~Hz}, 1 \mathrm{H}), 7.69-7.64(\mathrm{~m}, 1 \mathrm{H}), 7.24(\mathrm{~d}, J=8.4 \mathrm{~Hz}, 2 \mathrm{H}), 7.07(\mathrm{~d}, J=$ 
8.4 Hz, 2 H), 6.99-6.96 (m, $1 \mathrm{H}), 6.89$ (d, $J=8.0$ Hz, $1 \mathrm{H}), 6.06-6.05$ (m, $1 \mathrm{H}), 2.82-$ 2.76 (m, $1 \mathrm{H}), 2.40-2.36$ (m, $1 \mathrm{H}), 2.23-2.19$ (m, $3 \mathrm{H}), 1.98-1.95$ (m, $1 \mathrm{H}), 1.74-1.64$ $(\mathrm{m}, 1 \mathrm{H}), 0.94(\mathrm{t}, J=8.0 \mathrm{~Hz}, 9 \mathrm{H}), 0.60(\mathrm{q}, J=8.0 \mathrm{~Hz}, 6 \mathrm{H}) .{ }^{13} \mathrm{C}$ NMR $(100 \mathrm{MHz}$, $\left.\mathrm{CDCl}_{3}\right) \delta 163.9,152.1,147.8,143.8,139.3,136.4,135.5,128.0,120.9,118.2,111.4$, 39.5, 35.2, 30.2, 28.2, 7.5, 2.6. FTMS (ESI/Quadrupole): Calculated for $\mathrm{C}_{23} \mathrm{H}_{32} \mathrm{NOSi}$ $(\mathrm{M}+\mathrm{H})^{+}:$366.2248; Found: 366.2253.

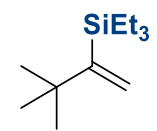

(3,3-Dimethylbut-1-en-2-yl)triethylsilane (53). ${ }^{11}$ The product $(48 \%$ yield) that determined by ${ }^{1} \mathrm{H}$ NMR couldn't be separated with Et ${ }_{3} \mathrm{Si}_{-}-\mathrm{SiEt}_{3}$ (Petroleum ether). ${ }^{1} \mathrm{H}$ NMR (400 MHz, $\left.\mathrm{CDCl}_{3}\right) \delta 5.74(\mathrm{~d}, J=1.6 \mathrm{~Hz}, 1 \mathrm{H}), 5.29$ (d, $\left.J=1.6 \mathrm{~Hz}, 1 \mathrm{H}\right), 1.07$ (s, $9 \mathrm{H}), 0.95-0.90(\mathrm{~m}, 19.5 \mathrm{H}), 0.67(\mathrm{q}, J=8.0 \mathrm{~Hz}, 6 \mathrm{H}), 0.52(\mathrm{q}, J=8.0 \mathrm{~Hz}, 7 \mathrm{H})$.<smiles>CCCC1=C(C)CCc2ccccc21</smiles>

Triethyl(2-methyl-3,4-dihydronaphthalen-1-yl)silane (54). The product (18.1 mg, $35 \%$ yield) as a colourless oil was purified with silica gel chromatography (Petroleum ether). ${ }^{1} \mathrm{H}$ NMR (400 MHz, $\left.\mathrm{CDCl}_{3}\right) \delta 7.16(\mathrm{t}, J=7.2 \mathrm{~Hz}, 1 \mathrm{H}), 7.12-7.08(\mathrm{~m}, 2 \mathrm{H})$, 7.07-7.04 (m, $1 \mathrm{H}), 2.60(\mathrm{t}, J=7.2 \mathrm{~Hz}, 2 \mathrm{H}), 2.11(\mathrm{t}, J=7.2 \mathrm{~Hz}, 2 \mathrm{H}), 2.05(\mathrm{~s}, 3 \mathrm{H})$, $0.94(\mathrm{t}, J=8.0 \mathrm{~Hz}, 9 \mathrm{H}), 0.83(\mathrm{q}, J=8.0 \mathrm{~Hz}, 6 \mathrm{H}) .{ }^{13} \mathrm{C} \mathrm{NMR}\left(100 \mathrm{MHz}, \mathrm{CDCl}_{3}\right) \delta 152.5$, $139.5,135.6,128.3,126.7,126.0,125.5,124.9,32.5,29.1,24.6$, 7.8, 6.0. HRMS (EI/TOF): Calculated for $\mathrm{C}_{17} \mathrm{H}_{26} \mathrm{Si}\left(\mathrm{M}^{+}\right)$: 294.1804; Found: 294.1809.

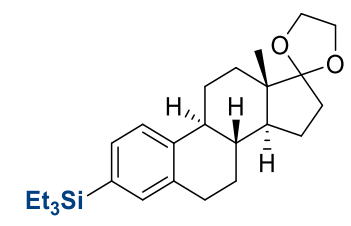

2-((8S,9S,14S)-6,7,8,9,11,12,13,14,15,16-Decahydrospiro[cyclopenta[a] phenan threne-17,2'-[1,3]dioxolan]-3-yl)triethylsilane (55). The reaction was carried out according to the general procedure on $0.2 \mathrm{mmol}$ scale, and $10 \mathrm{~mol} \% \mathrm{FeBr}_{3}, 10 \mathrm{~mol} \%$ 
dtbpy was used.The product $\mathbf{5 5}$ (48 $\mathrm{mg}, 58 \%$ yield) as a colourless oil was purified with silica gel chromatography (Petroleum ether). ${ }^{1} \mathrm{H}$ NMR (400 MHz, $\left.\mathrm{CDCl}_{3}\right) \delta$ 7.34-7.27 (m, 2 H), 7.22 (s, $1 \mathrm{H}), 4.06-3.79$ (m, $4 \mathrm{H}), 2.96-2.83(\mathrm{~m}, 2 \mathrm{H}), 2.46-2.26(\mathrm{~m}, 2 \mathrm{H})$, 2.16-2.01 (m, 1 H), 2.00-1.74 (m, 3 H), 1.73-1.24 (m, 7 H), 1.00 (t, J = 7.9 Hz, 9 H), $0.91(\mathrm{~s}, 3 \mathrm{H}), 0.80(\mathrm{q}, J=7.9 \mathrm{~Hz}, 6 \mathrm{H}) .{ }^{13} \mathrm{C} \mathrm{NMR}\left(100 \mathrm{MHz}, \mathrm{CDCl}_{3}\right): \delta$ 141.0, 135.8, 135.0, 134.0, 131.5, 124.6, 119.4, 65.2, 64.6, 49.6, 46.2, 44.2, 38.8, 34.2, 30.8, 29.6, 27.1, 25.7, 22.4, 14.3, 7.5, 3.4. FTMS (ESI/Quadrupole): Calculated for $\mathrm{C}_{26} \mathrm{H}_{41} \mathrm{O}_{2} \mathrm{Si}$ $(\mathrm{M}+\mathrm{H})^{+}:$413.2870; Found: 413.2857.

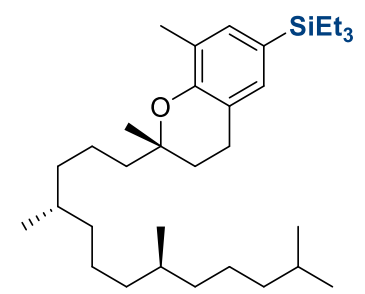

((S)-2,8-Dimethyl-2-((4S,8S)-4,8,12-trimethyltridecyl)chroman-6yl)triethylsilane

(56). The reaction was carried out according to the general procedure on $0.2 \mathrm{mmol}$ scale. $10 \mathrm{~mol} \% \mathrm{FeBr}_{3}, 20 \mathrm{~mol} \%$ dtbpy was used. The product 56 (48 $\mathrm{mg}, 48 \%$ yield) as a colourless oil was purified with silica gel chromatography (Petroleum ether). ${ }^{1} \mathrm{H}$ NMR $\left(600 \mathrm{MHz}, \mathrm{CDCl}_{3}\right) \delta 7.06(\mathrm{~s}, 1 \mathrm{H}), 7.00(\mathrm{~s}, 1 \mathrm{H}), 2.81-2.70(\mathrm{~m}, 2 \mathrm{H}), 2.17$ (s, $\left.3 \mathrm{H}\right), 1.87-$ 1.81 (m, $1 \mathrm{H}), 1.79-1.73$ (m, $1 \mathrm{H}), 1.62-1.50$ (m, $4 \mathrm{H}), 1.45-1.35$ (m, $2 \mathrm{H}), 1.35-1.23$ $(\mathrm{m}, 13 \mathrm{H}), 1.20-1.03(\mathrm{~m}, 3 \mathrm{H}), 0.98$ (t, $J=7.9 \mathrm{~Hz}, 9 \mathrm{H}), 0.90-0.84(\mathrm{~m}, 14 \mathrm{H}), 0.76$ (q, $J$ $=7.9 \mathrm{~Hz}, 6 \mathrm{H}) .{ }^{13} \mathrm{C} \mathrm{NMR}\left(100 \mathrm{MHz}, \mathrm{CDCl}_{3}\right): \delta 153.0,134.1,133.2,125.8,125.4,119.8$, 76.1, 40.5, 39.4, 37.4, 32.8, 32.7, 31.2, 29.7, 28.0, 24.8, 24.4, 22.7, 22.3, 21.0, 19.8, 19.7, 16.2, 7.6, 3.6. FTMS (ESI/Quadrupole): Calculated for $\mathrm{C}_{33} \mathrm{H}_{61} \mathrm{OSi}(\mathrm{M}+\mathrm{H})^{+}$: 501.4486; Found: 501.4469.<smiles>CCCc1cc(-c2cccc(OC)c2)c2ccccc2c1</smiles>

Triethyl(4-(3-methoxyphenyl)naphthalen-2-yl)silane (57). The product 57 (75 mg, $72 \%$ yield) as a colourless oil was purified with silica gel chromatography (Petroleum 
ether). ${ }^{1} \mathrm{H}$ NMR (400 MHz, $\left.\mathrm{CDCl}_{3}\right) \delta 8.04$ (s, $\left.1 \mathrm{H}\right), 7.97-7.93$ (m, $\left.2 \mathrm{H}\right)$, 7.56-7.43 (m, $4 \mathrm{H}), 7.15(\mathrm{~d}, J=7.6 \mathrm{~Hz}, 1 \mathrm{H}), 7.11(\mathrm{~d}, J=2.8 \mathrm{~Hz}, 1 \mathrm{H}), 7.16-7.01(\mathrm{~m}, 1 \mathrm{H}), 3.90(\mathrm{~s}, 3$ H), $1.06(\mathrm{q}, J=7.9 \mathrm{~Hz}, 9 \mathrm{H}), 0.94(\mathrm{t}, J=7.9 \mathrm{~Hz}, 6 \mathrm{H}) .{ }^{13} \mathrm{C} \mathrm{NMR}\left(100 \mathrm{MHz}, \mathrm{CDCl}_{3}\right): \delta$ 159.4, 142.5, 138.8, 134.7, 134.6, 133.1, 131.7, 131.5, 131.4, 129.2, 128.3, 126.3, 125.8, 122.6, 115.8, 112.5, 55.2, 7.5, 3.4. FTMS (ESI/Quadrupole): Calculated for $\mathrm{C}_{23} \mathrm{H}_{29} \mathrm{OSi}$ $(\mathrm{M}+\mathrm{H})^{+}:$349.1982; Found: 349.1991.<smiles>CCCc1cc(-c2ccc(Br)cc2)c2ccccc2c1</smiles>

Triethyl(4-(4-(trifluoromethyl)phenyl)naphthalen-2-yl)silane (58). The product 58 (51 mg, $44 \%$ yield, m.p. $45.3-48.9{ }^{\circ} \mathrm{C}$ ) as a white solid was purified with silica gel chromatography (Petroleum ether). ${ }^{1} \mathrm{H}$ NMR $\left(400 \mathrm{MHz}, \mathrm{CDCl}_{3}\right) \delta 8.06$ (s, $1 \mathrm{H}$ ), 7.96$7.93(\mathrm{~d}, J=8.3 \mathrm{~Hz}, 1 \mathrm{H}), 7.82(\mathrm{~d}, J=8.3 \mathrm{~Hz}, 1 \mathrm{H}), 7.78(\mathrm{~d}, J=8.0 \mathrm{~Hz}, 2 \mathrm{H}), 7.65(\mathrm{~d}, J$ $=8.0 \mathrm{~Hz}, 2 \mathrm{H}), 7.55-7.45(\mathrm{~m}, 2 \mathrm{H}), 7.49(\mathrm{~s}, 1 \mathrm{H}), 1.04(\mathrm{q}, J=7.6 \mathrm{~Hz}, 9 \mathrm{H}), 0.94-0.87$ (t, $J=7.6 \mathrm{~Hz}, 6 \mathrm{H}) .{ }^{13} \mathrm{C} \mathrm{NMR}\left(100 \mathrm{MHz}, \mathrm{CDCl}_{3}\right): \delta 144.8,135.3,135.2,134.7,131.7$, 131.6, 131.4, 130.4, 128.5, 126.0, 126.7, 125.9, $125.2\left(\mathrm{q},{ }^{3} J_{\mathrm{CF}}=3.7 \mathrm{~Hz}\right), 7.5,3.3$. FTMS (ESI/Quadrupole): Calculated for $\mathrm{C}_{23} \mathrm{H}_{25} \mathrm{~F}_{3} \mathrm{Si}\left(\mathrm{M}^{+}\right)$: 386.1678; Found: 386.1673. 


\section{Reference}

1. Liu, Q.; Sun, B.; Liu, Z.; Kao, Y.; Dong, B.-W.; Jiang, S.-D.; Li, F.; Liu, G.; Yang, Y.; Mo, F. Chem. Sci. 2018, 9, 8731.

2. (a) Yasui, K.; Chatani, N.; Tobisu, M. Org. Lett. 2018, 20, 2108. (b) Quasdorf, K. W.; Riener, M.; Petrova, K. V.; Garg, N. K. J. Am. Chem. Soc. 2009, 131, 17748.

3. Xu, L.; Li, B.-J.; Wu, Z.-H.; Lu, H.-Y.; Guan, B.-T.; Wang, B.-Q.; Zhao, K.-Q.; Shi, Z.-J. Org. Lett. 2010, 12, 884.

4. Boultadakis-Arapinis, B.; Hopkinson, M. N.; Glorius, F. Org. Lett. 2016, 16, 1630.

5. Zarate, C.; Martin, R. J. Am. Chem. Soc. 2014, 136, 2236.

6. Murai, M.; Takami, K.; Takai, K. Chem. Eur. J. 2015, 21, 4566.

7. Choi, G.; Tsurugi, H.; Mashima, K. J. Am. Chem. Soc. 2013, 135, 13149.

8. Wiensch, E. M.; Todd, D. P.; Montgomery, J. ACS Catal. 2017, 7, 5568.

9. Guo, L.; Chatupheeraphat, A.; Rueping, M. Angew. Chem. Int. Ed. 2016, 55, 11810.

10. Yoshinori, Y. J. Org. Chem. 2005, 70, 9607.

11. (a) Gee, J. C.; Fuller, B. A.; Lockett, H.-M.; Sedghi, G.; Robertson, C. M.; Luzyanin, K. V. Chem. Commun. 2018, 54, 9450. (b) Itazaki, M.; Ueda, K.; Nakazawa, H. Angew. Chem. Int. Ed. 2009, 48, 3313. 


\section{Spectra of Products 3-58:}

Triethyl(naphthalen-1-yl)silane (3)
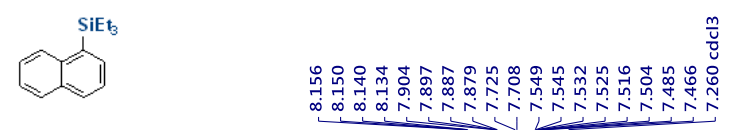

${ }^{1} \mathrm{H}$ NMR (400 MHz, $\left.\mathrm{CDCl}_{3}\right)$
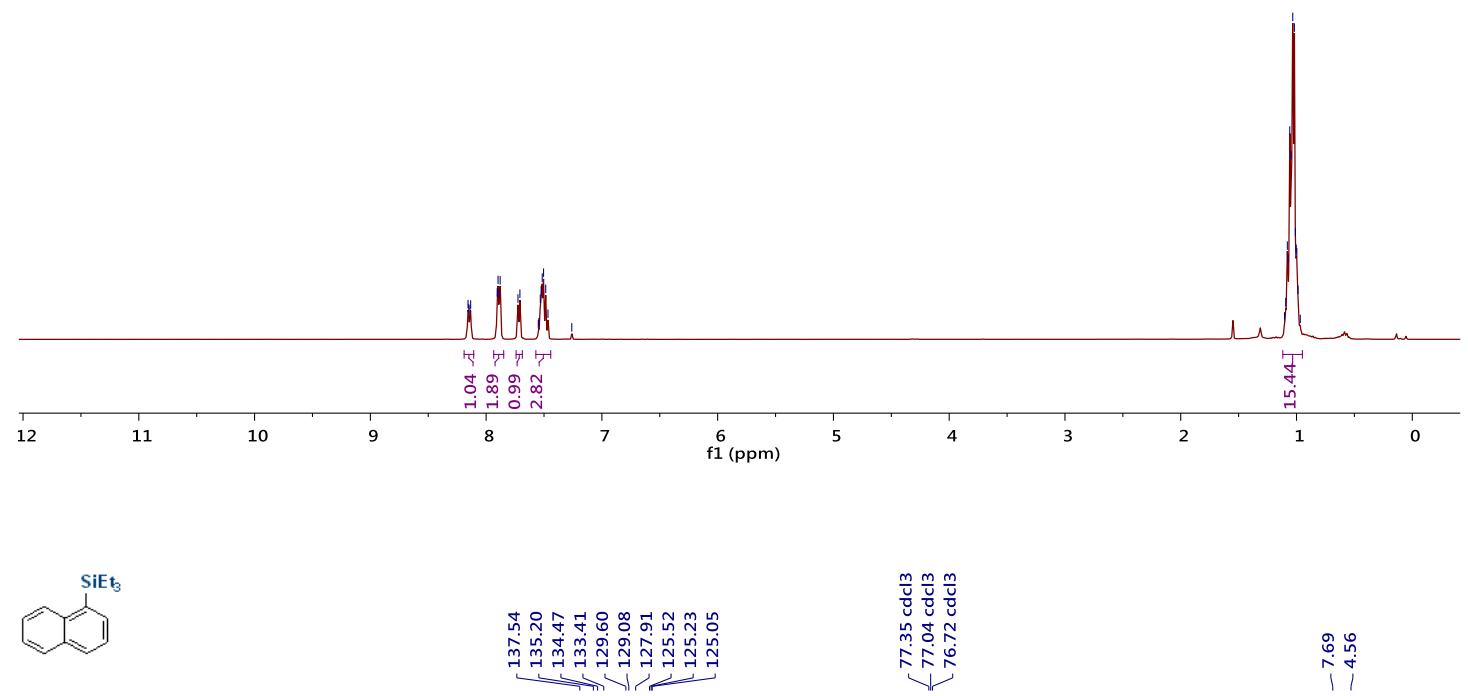

${ }^{13} \mathrm{C}$ NMR $\left(100 \mathrm{MHz}, \mathrm{CDCl}_{3}\right)$

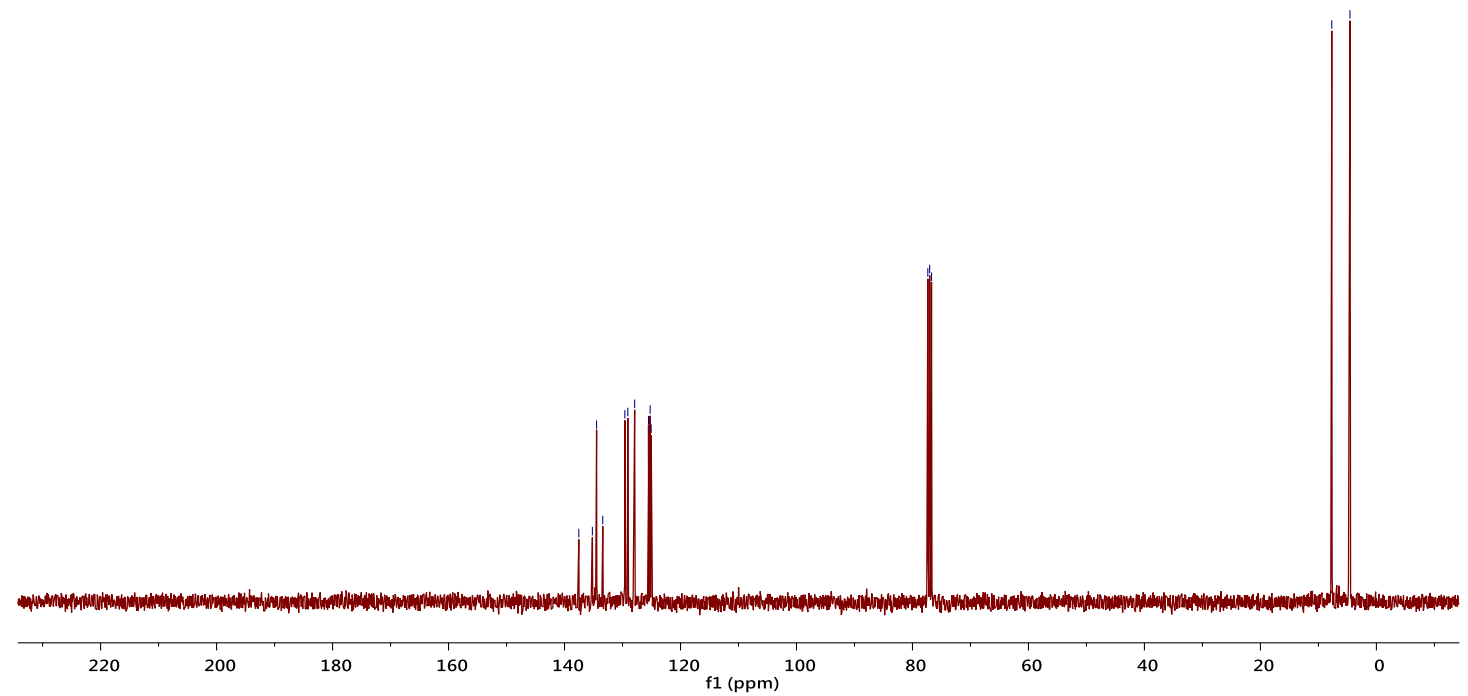


Triethyl(naphthalen-2-yl)silane (4)
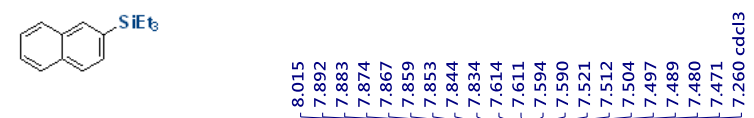

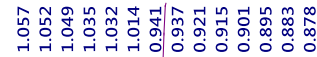

${ }^{1} \mathrm{H} \mathrm{NMR}\left(400 \mathrm{MHz}, \mathrm{CDCl}_{3}\right)$
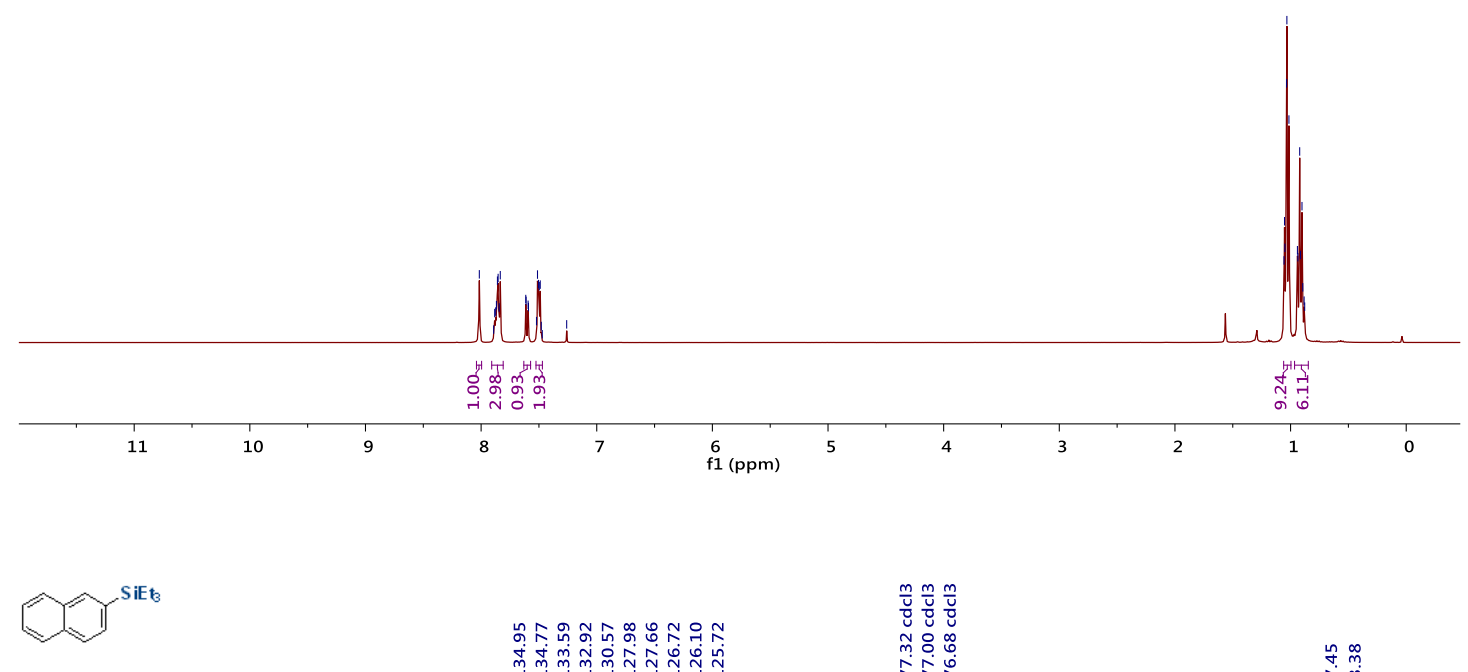

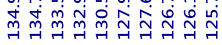

${ }^{13} \mathrm{C} \mathrm{NMR}\left(100 \mathrm{MHz}, \mathrm{CDCl}_{3}\right)$

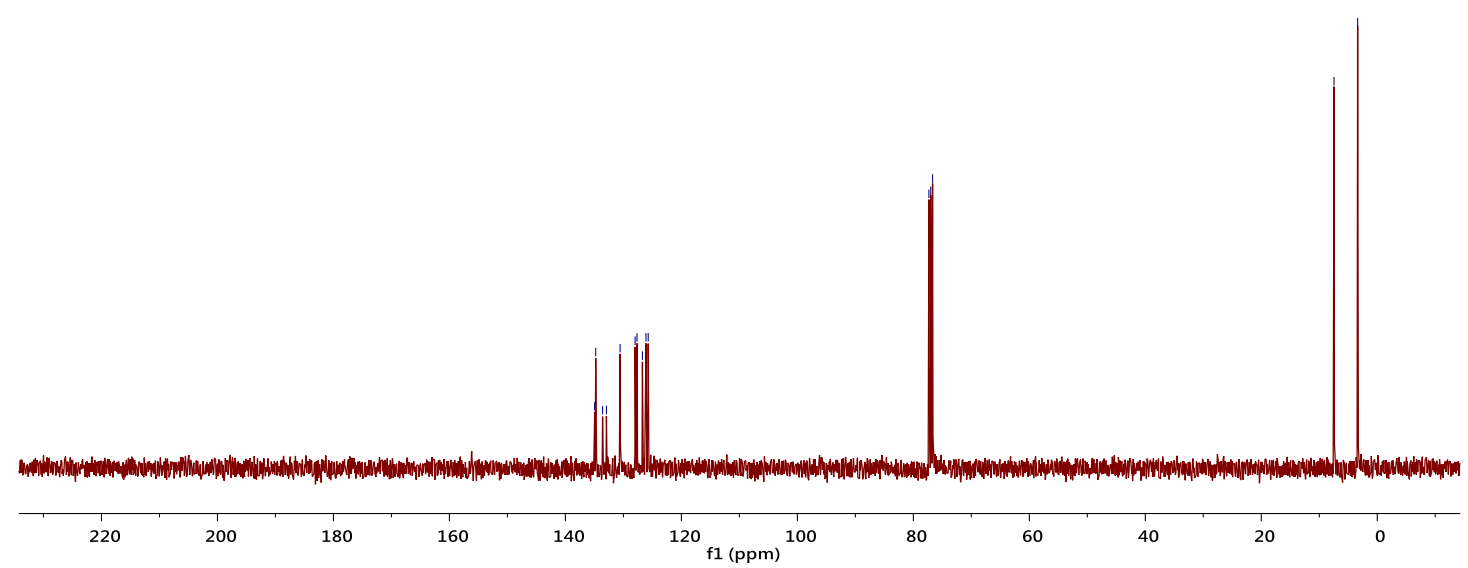


Triethyl(4-methoxynaphthalen-1-yl)silane (5)

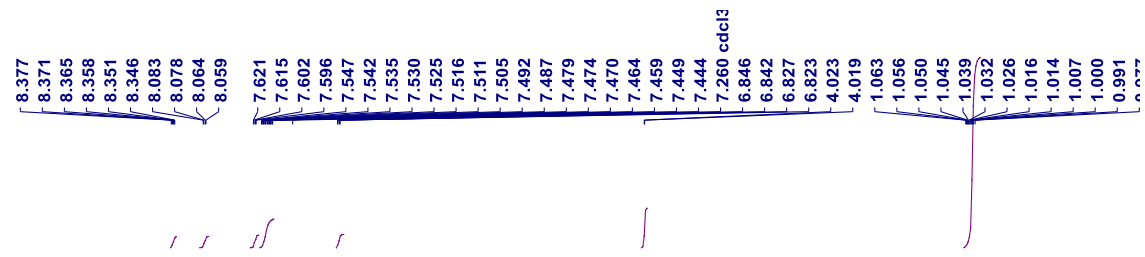

${ }^{1} \mathrm{H}$ NMR $\left(400 \mathrm{MHz}, \mathrm{CDCl}_{3}\right)$
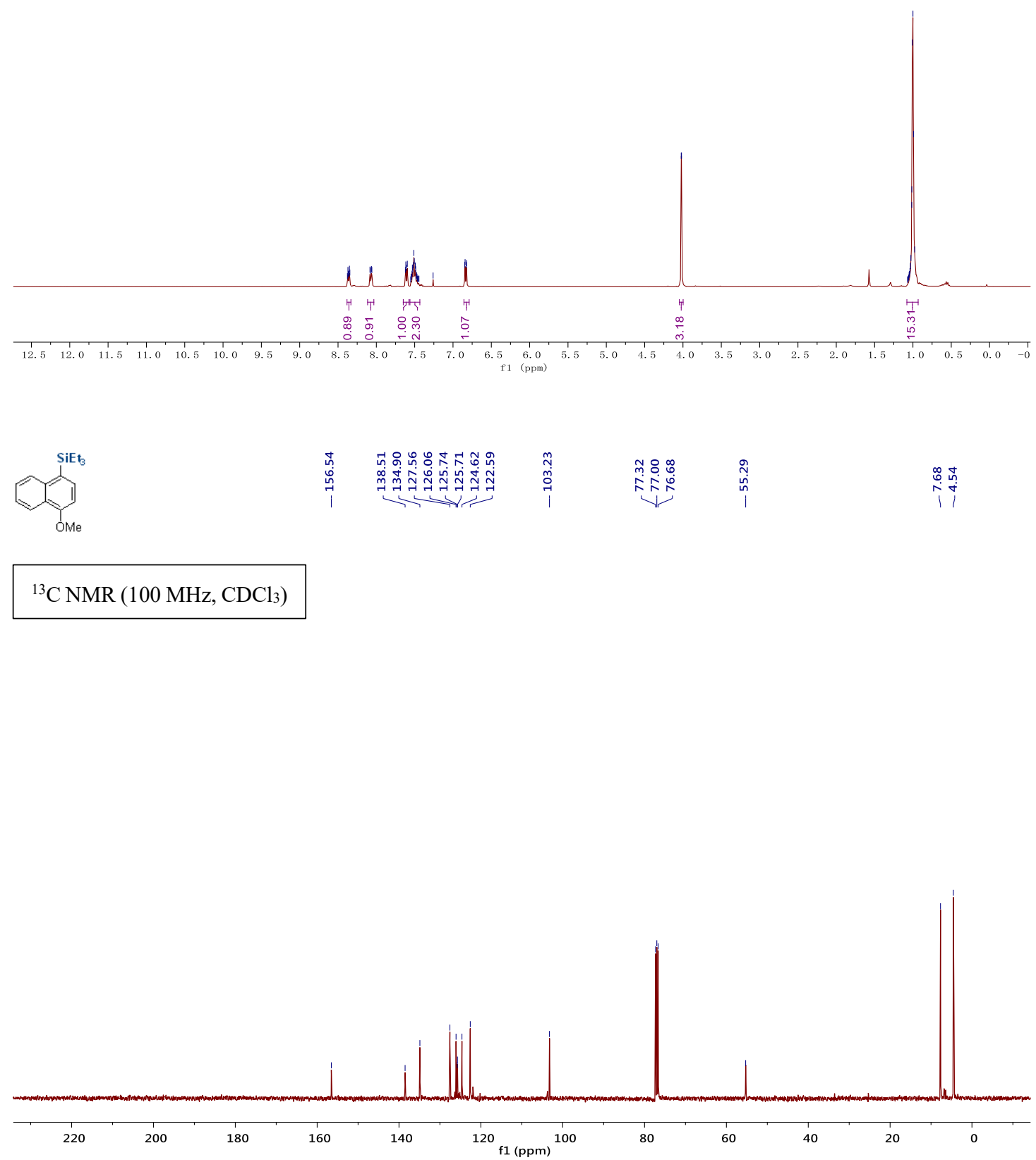
Triethyl(7-methoxynaphthalen-2-yl)silane (6)
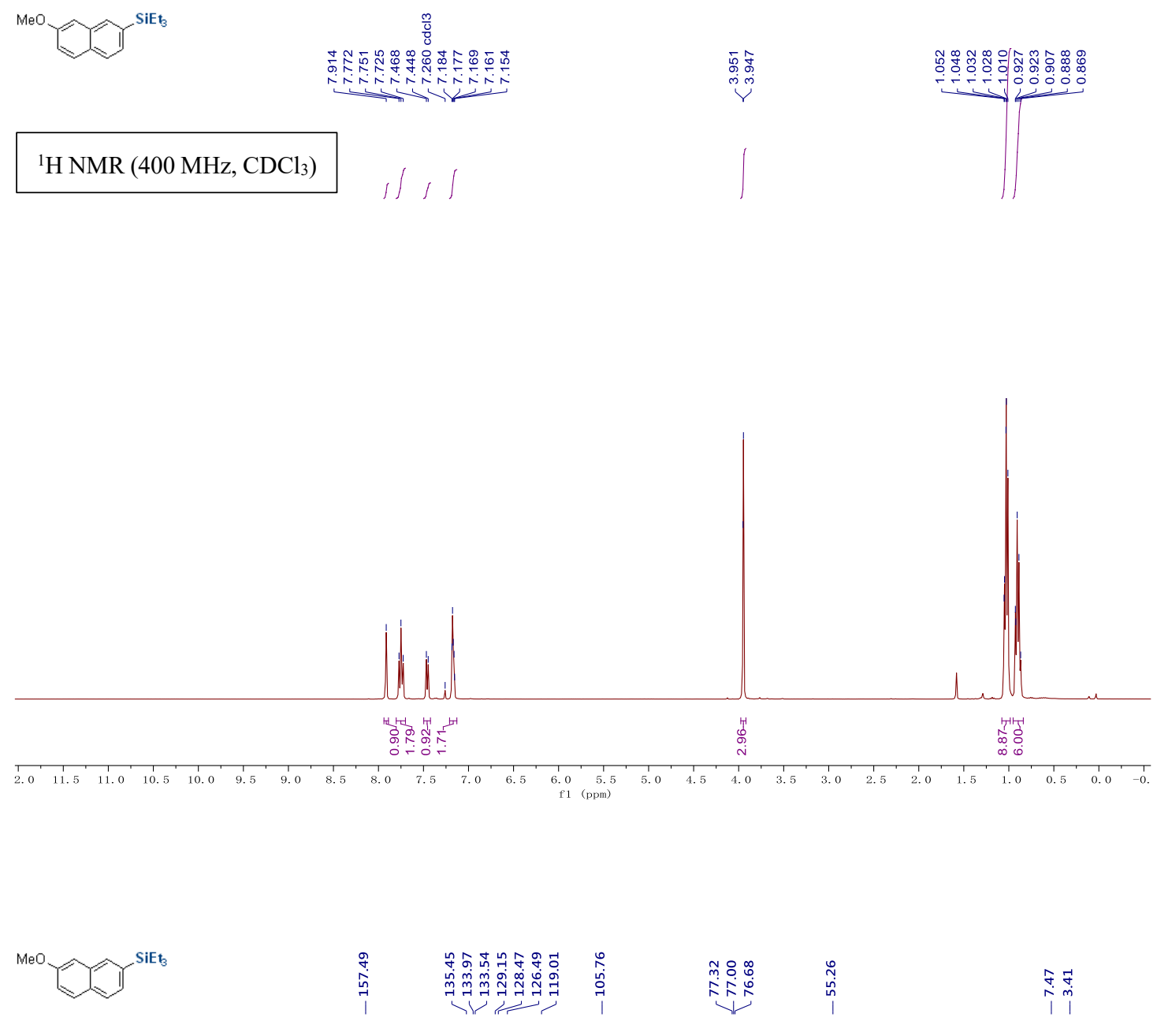

${ }^{13} \mathrm{C} \mathrm{NMR}\left(100 \mathrm{MHz}, \mathrm{CDCl}_{3}\right)$

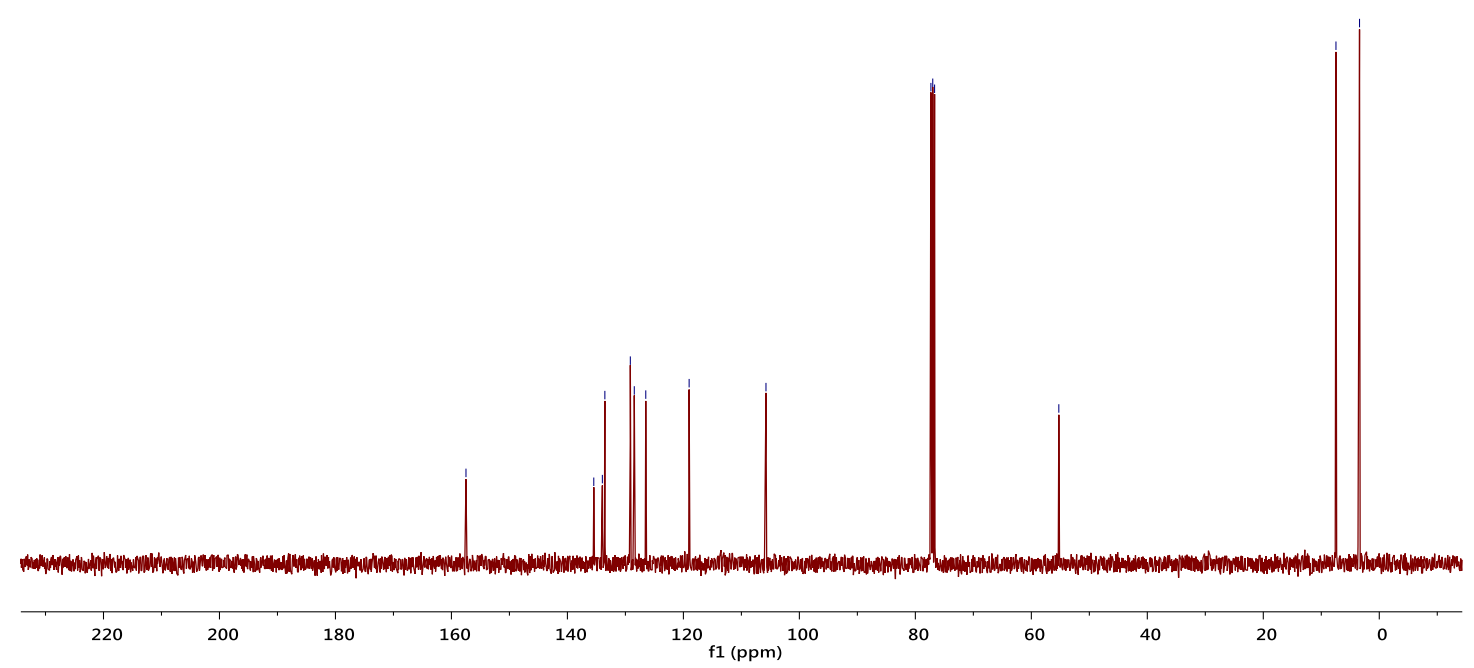


tert-Butyldimethyl((6-(triethylsilyl)naphthalen-2-yl)oxy)silane (7)

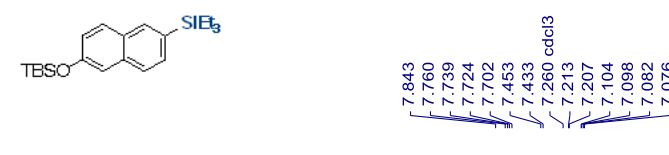

${ }^{1} \mathrm{H}$ NMR (400 MHz, $\left.\mathrm{CDCl}_{3}\right)$
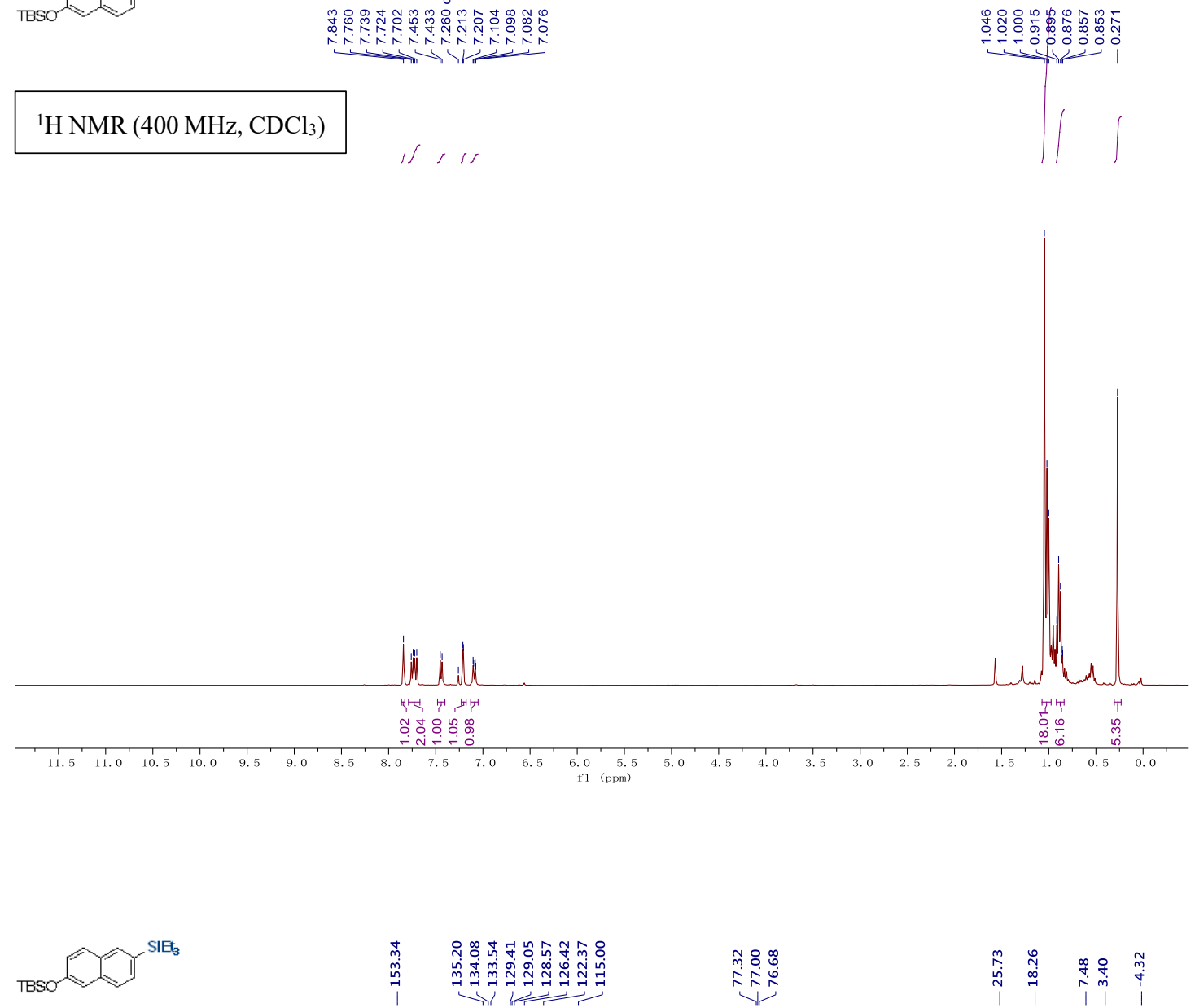

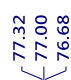

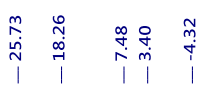

${ }^{13} \mathrm{C}$ NMR (100 MHz, $\mathrm{CDCl}_{3}$ )

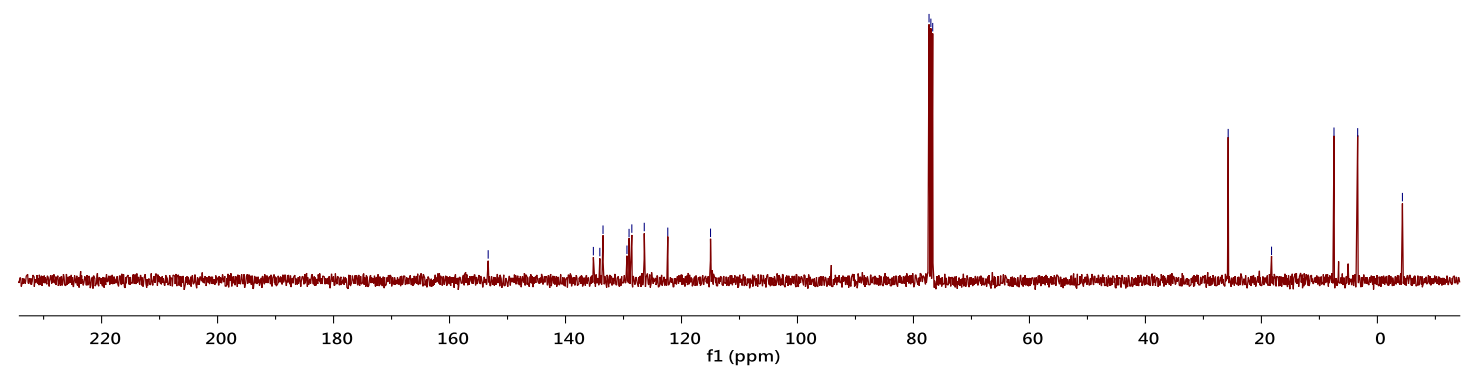


Triethyl(6-(4-(trifluoromethyl)phenyl)naphthalen-2-yl)silane (8)
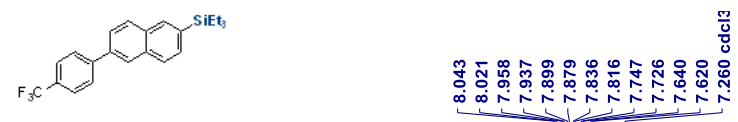

\section{$\frac{0}{50}$}

${ }^{1} \mathrm{H} \mathrm{NMR}\left(400 \mathrm{MHz}, \mathrm{CDCl}_{3}\right)$
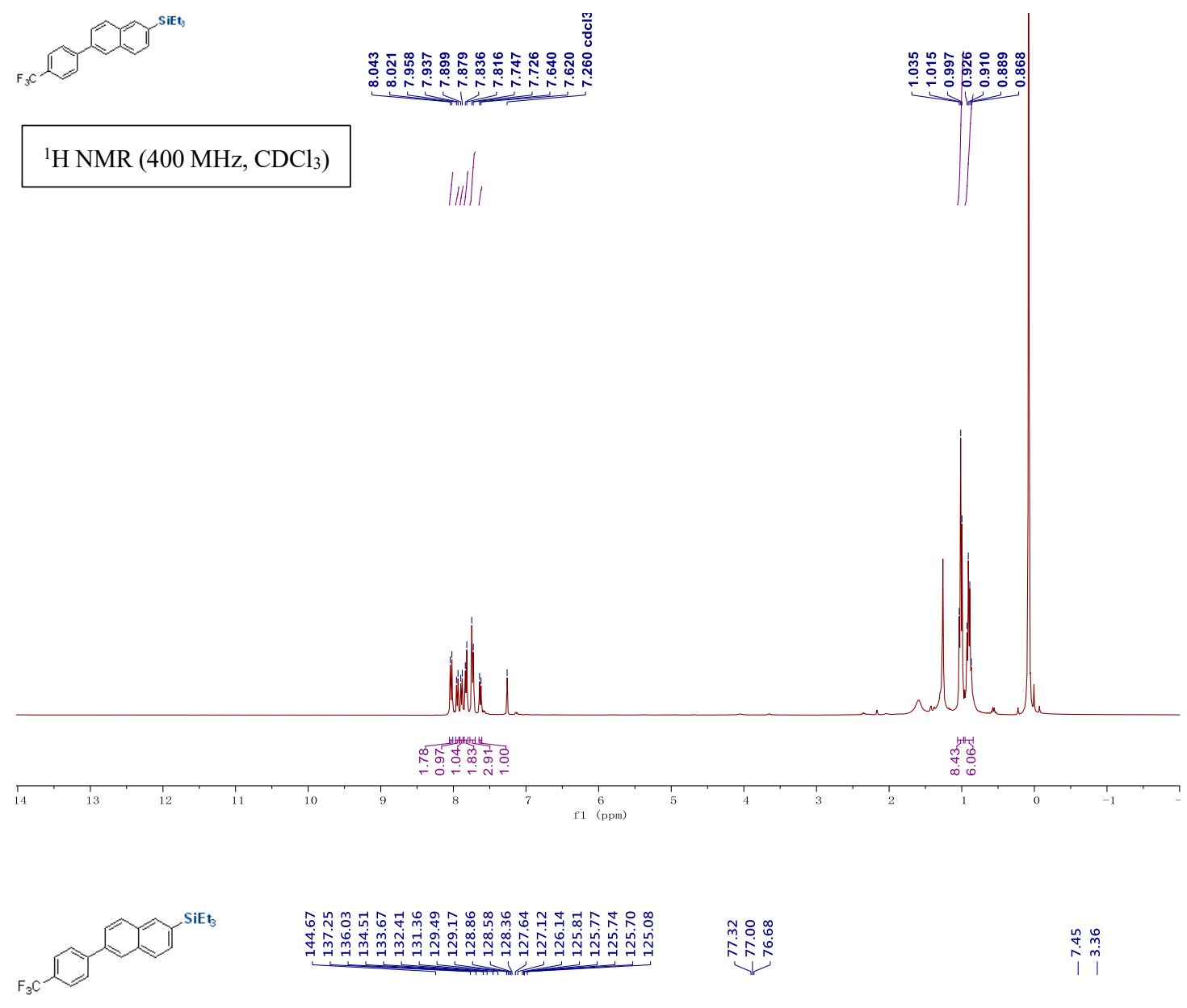

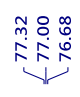

${ }^{13} \mathrm{C} \mathrm{NMR}\left(100 \mathrm{MHz}, \mathrm{CDCl}_{3}\right)$

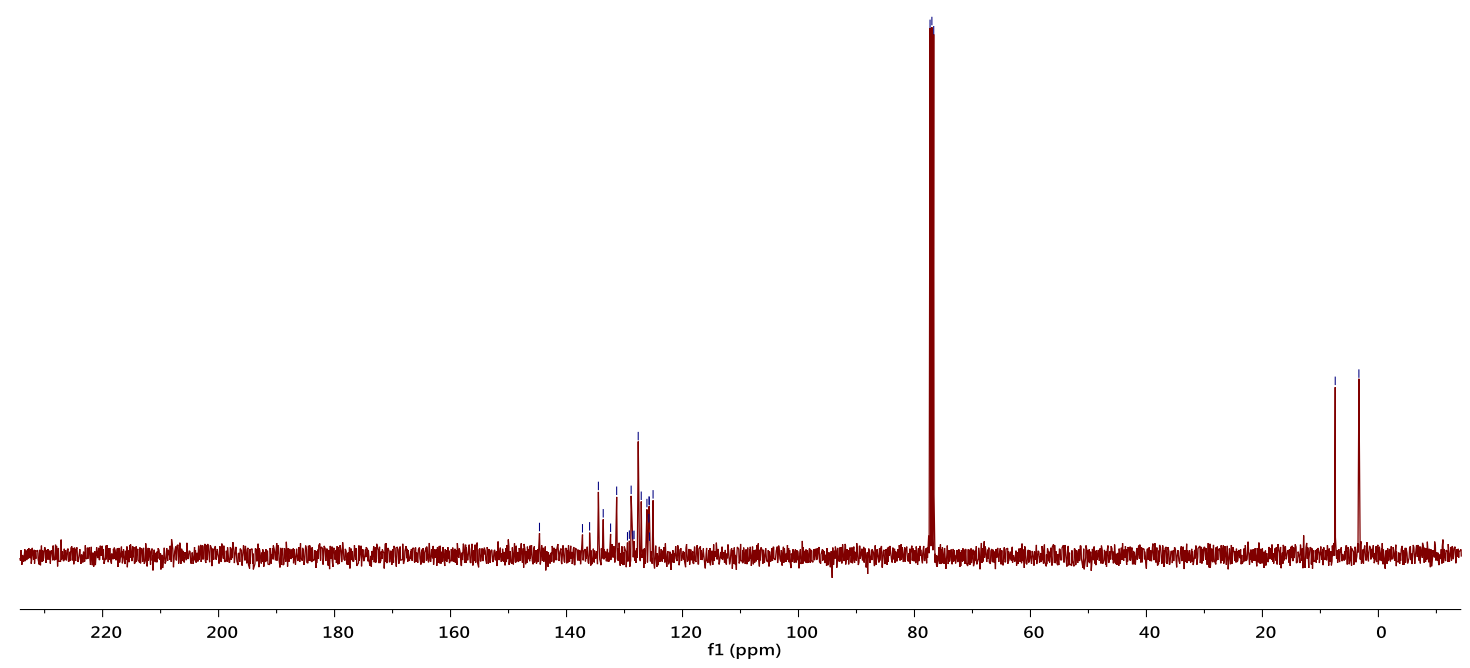


Triethyl(6-(p-tolyl)naphthalen-2-yl)silane (9)
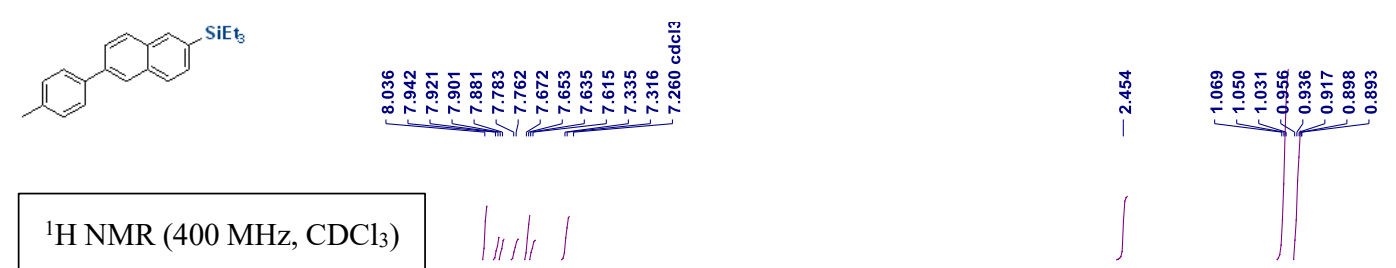

${ }^{1} \mathrm{H}$ NMR (400 MHz, $\left.\mathrm{CDCl}_{3}\right)$
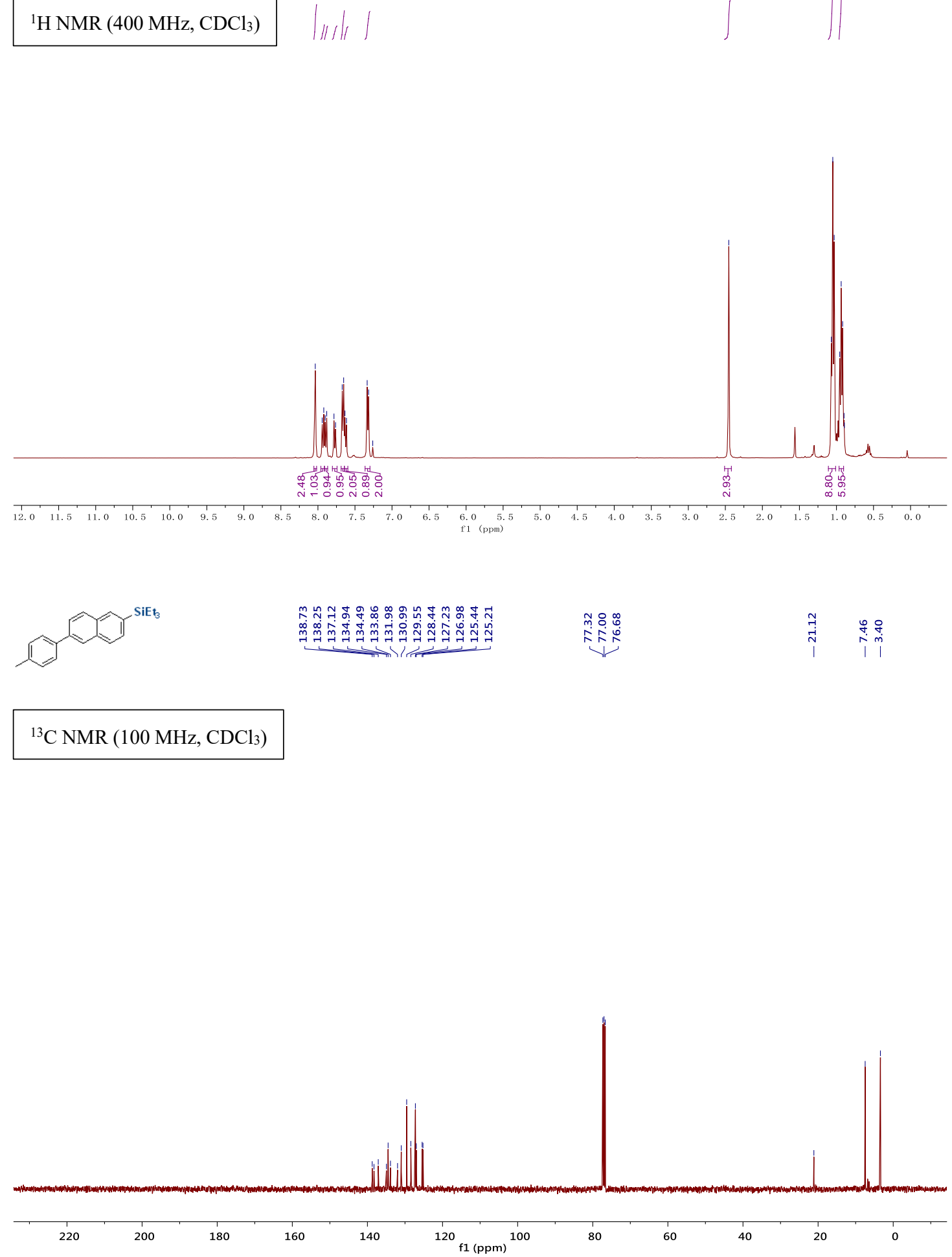
Triethyl(6-(4-methoxyphenyl)naphthalen-2-yl)silane (10)
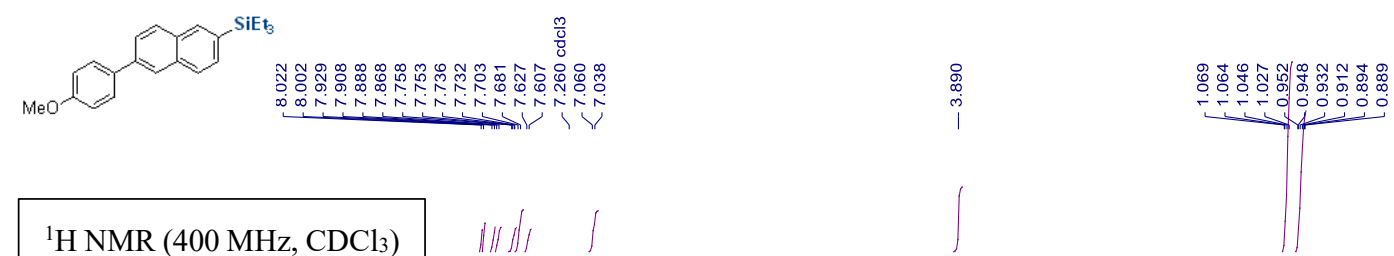

${ }^{1} \mathrm{H}$ NMR (400 MHz, $\left.\mathrm{CDCl}_{3}\right)$

\|\|$\|$
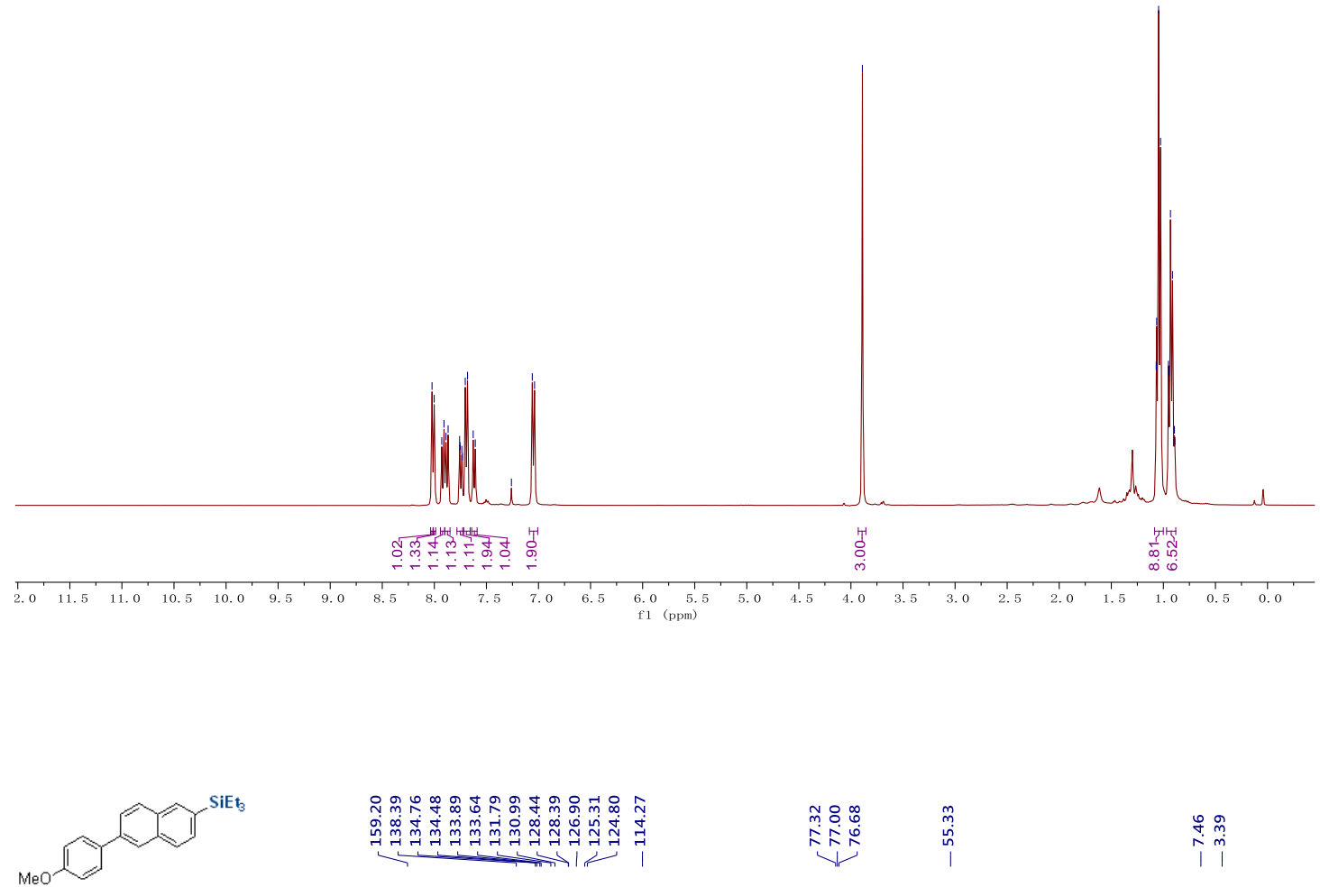

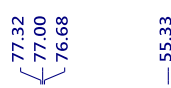

${ }^{13} \mathrm{C} \mathrm{NMR}\left(100 \mathrm{MHz}, \mathrm{CDCl}_{3}\right)$

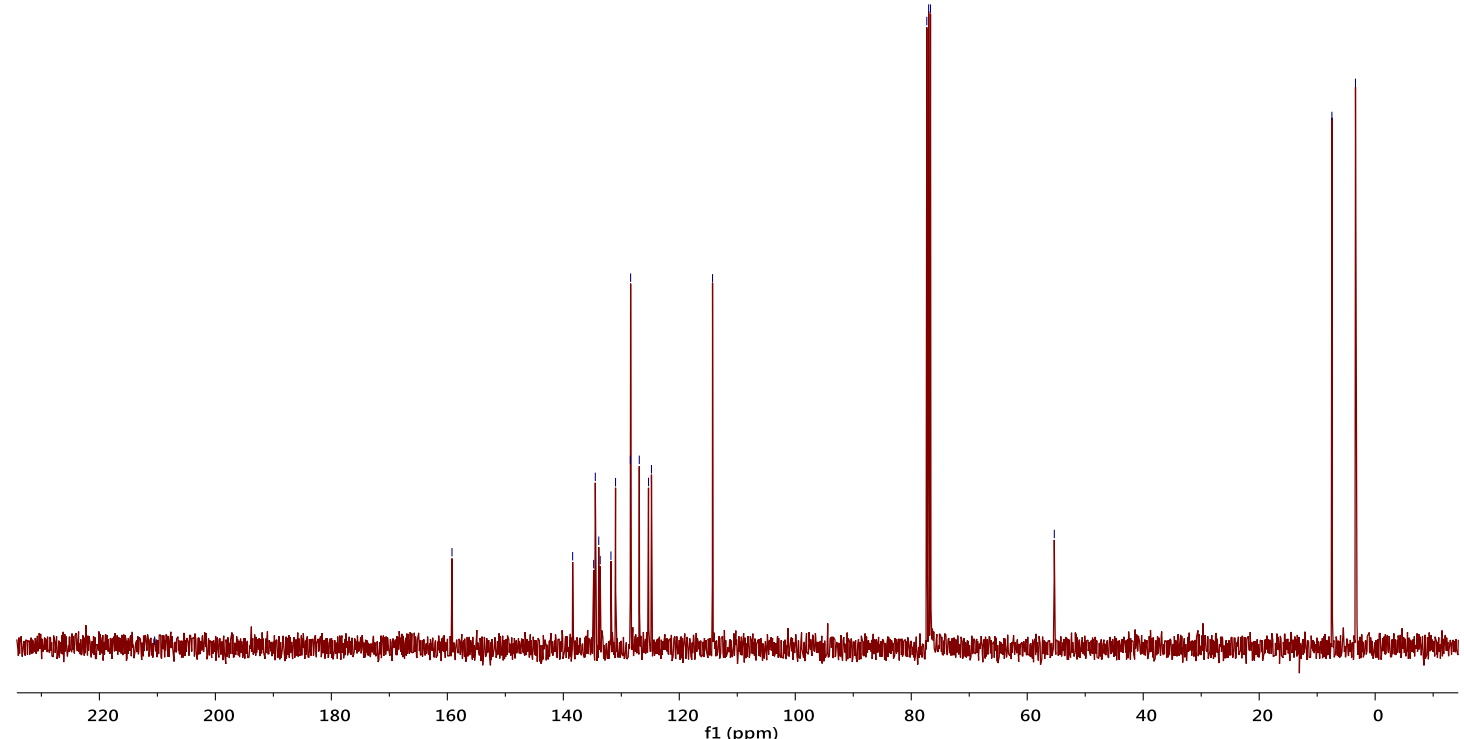


Anthracen-2-yltriethylsilane (11)

OSils

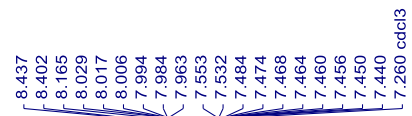

${ }^{1} \mathrm{H}$ NMR $\left(400 \mathrm{MHz}, \mathrm{CDCl}_{3}\right)$

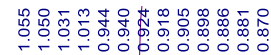

II i /
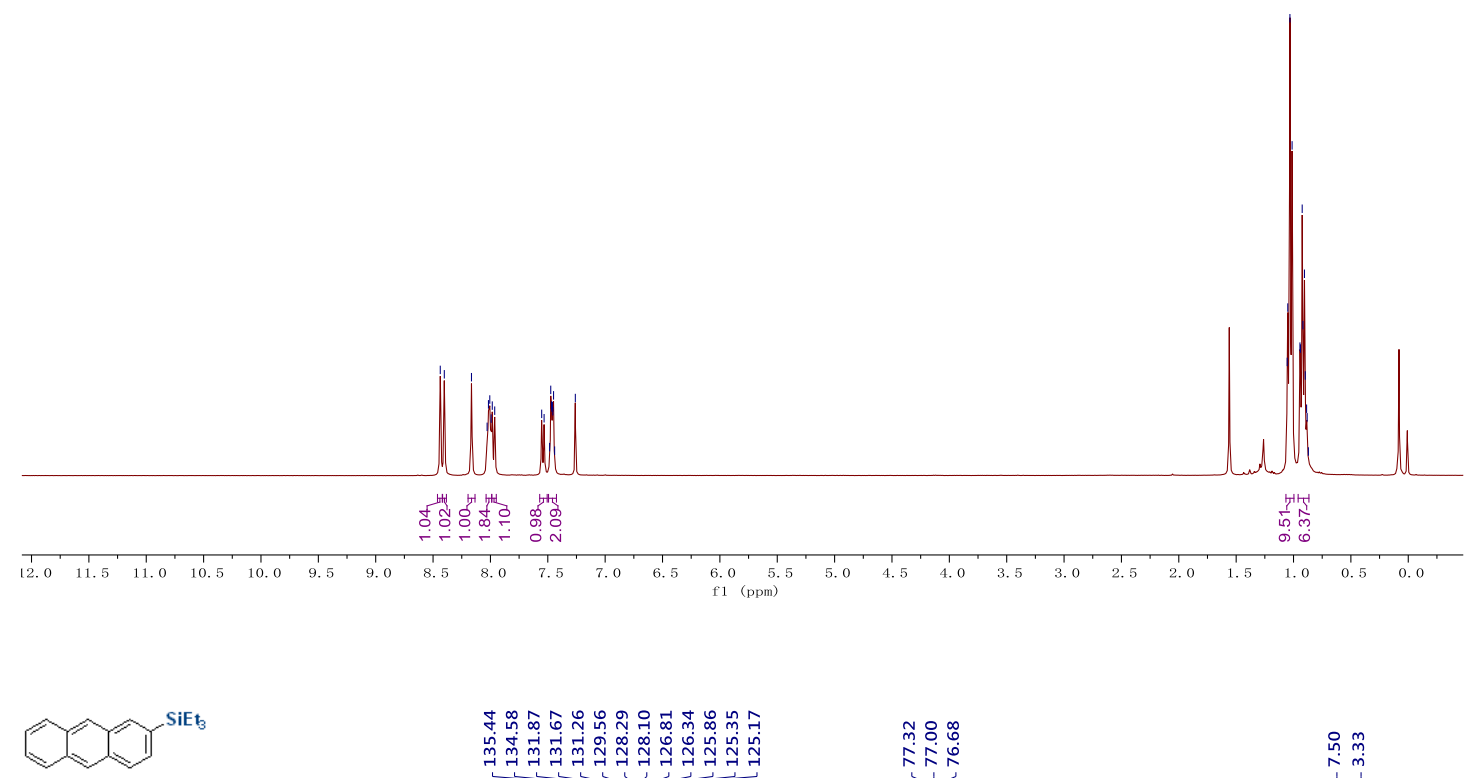

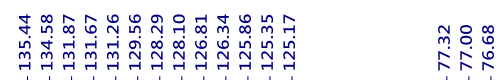

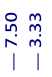

${ }^{13} \mathrm{C}$ NMR $\left(100 \mathrm{MHz}, \mathrm{CDCl}_{3}\right)$

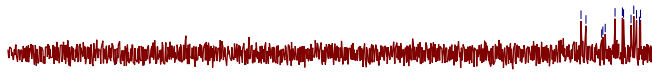

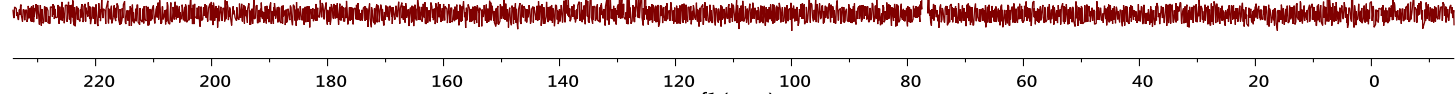


Triethyl(phenanthren-9-yl)silane (12)
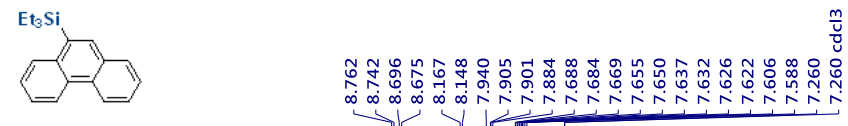

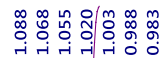

$\overbrace{}^{\infty}$

'H NMR (400 MHz, CDCl $)$
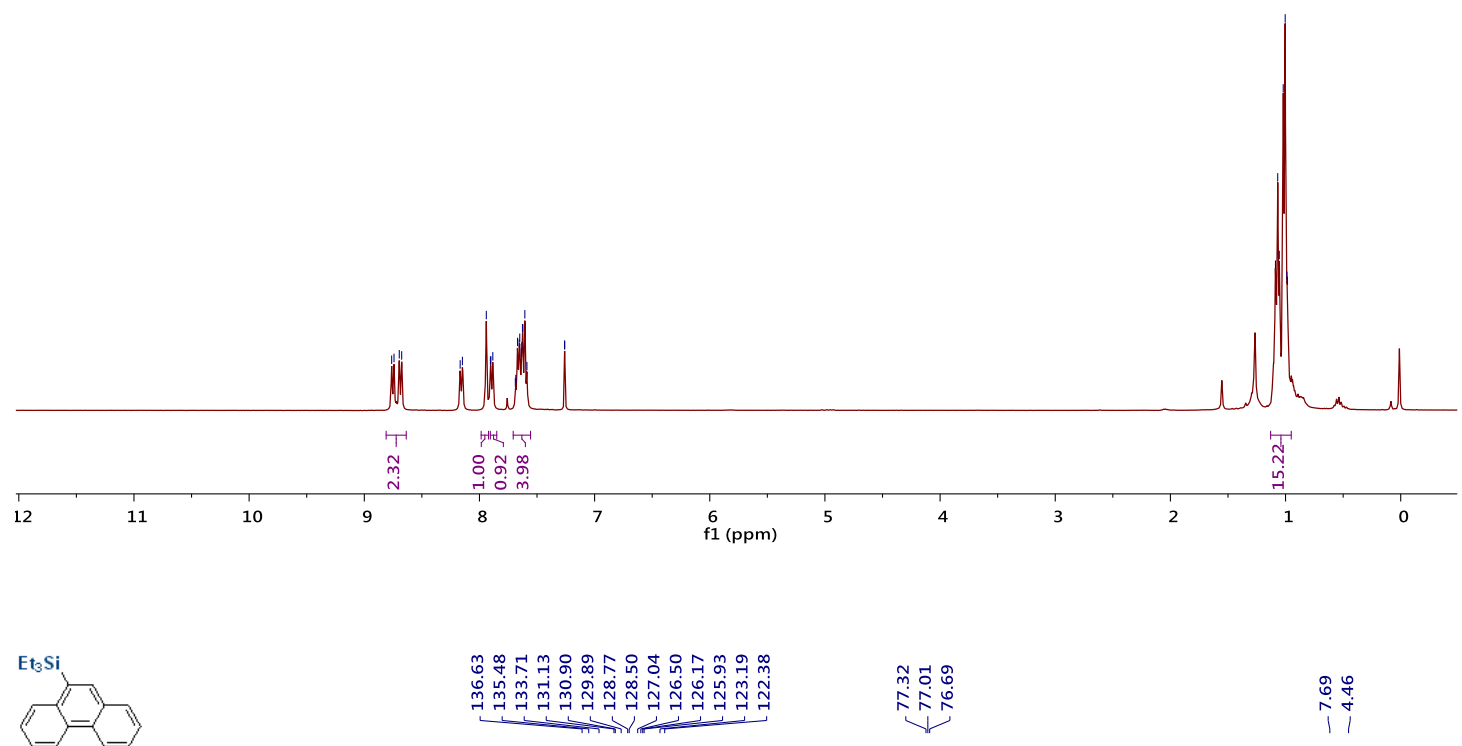

${ }^{13} \mathrm{C}$ NMR (100 MHz, $\left.\mathrm{CDCl}_{3}\right)$

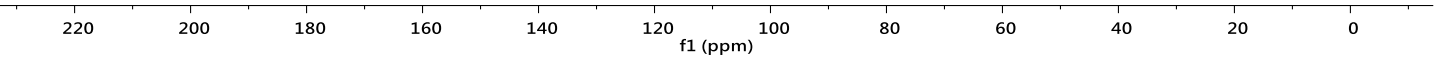


(1,2-dihydroacenaphthylen-5-yl)triethylsilane (13)
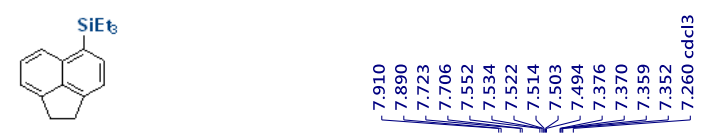

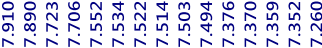

${ }^{1} \mathrm{H}$ NMR (400 MHz, $\left.\mathrm{CDCl}_{3}\right)$
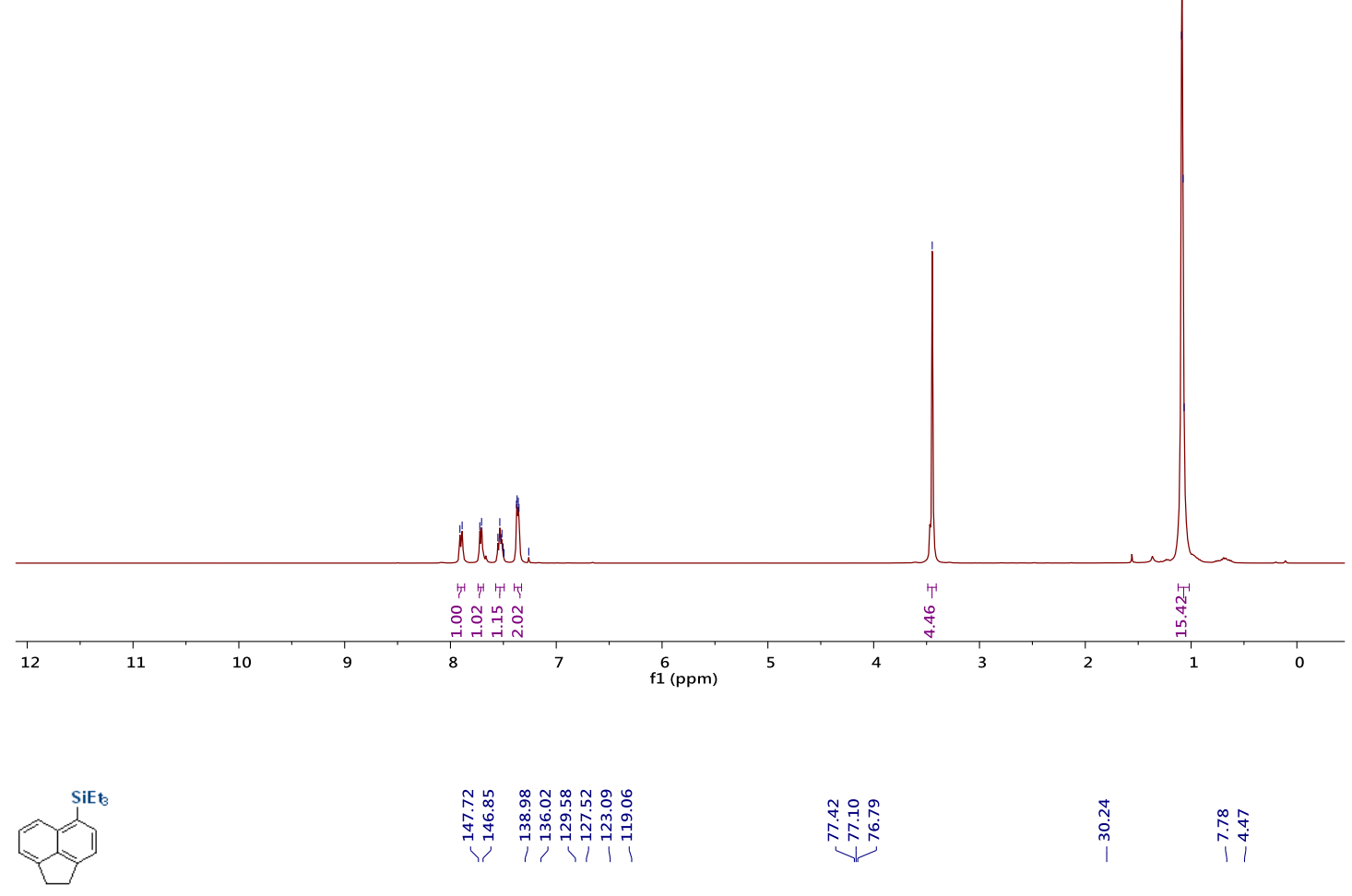

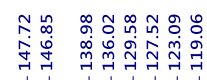

采㝊究

${ }^{13} \mathrm{C} \mathrm{NMR}\left(100 \mathrm{MHz}, \mathrm{CDCl}_{3}\right)$

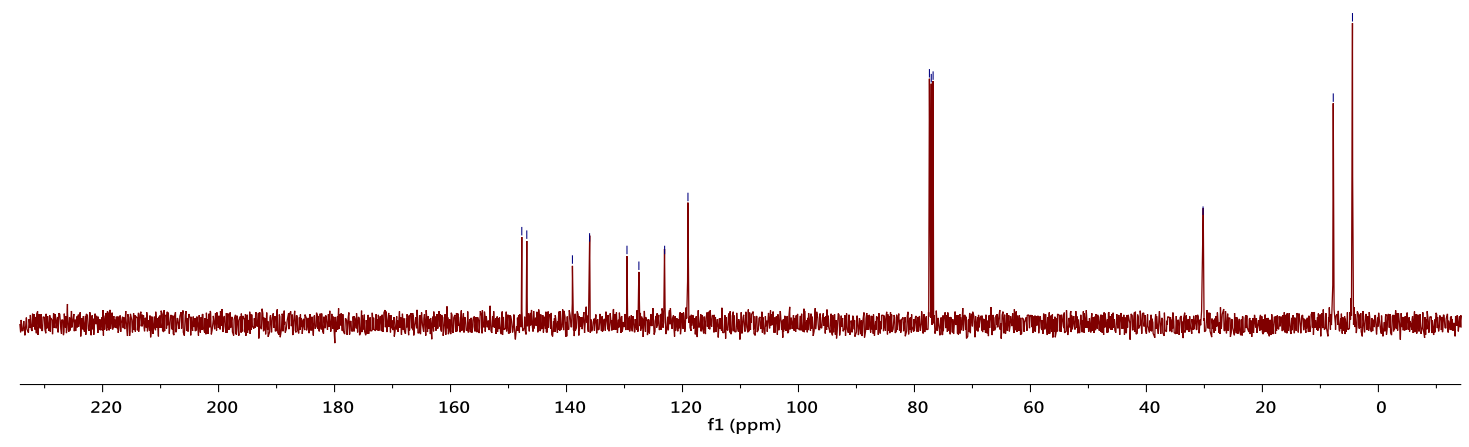


Triethyl(pyren-1-yl)silane (14)

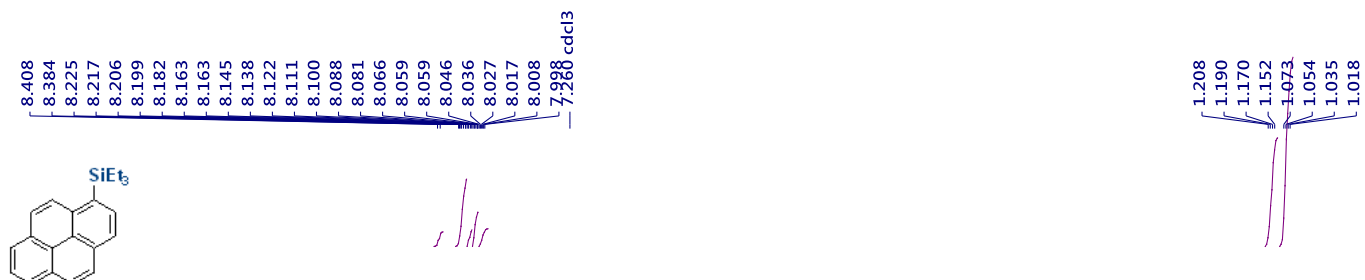

${ }^{1} \mathrm{H} \mathrm{NMR}\left(400 \mathrm{MHz}, \mathrm{CDCl}_{3}\right)$
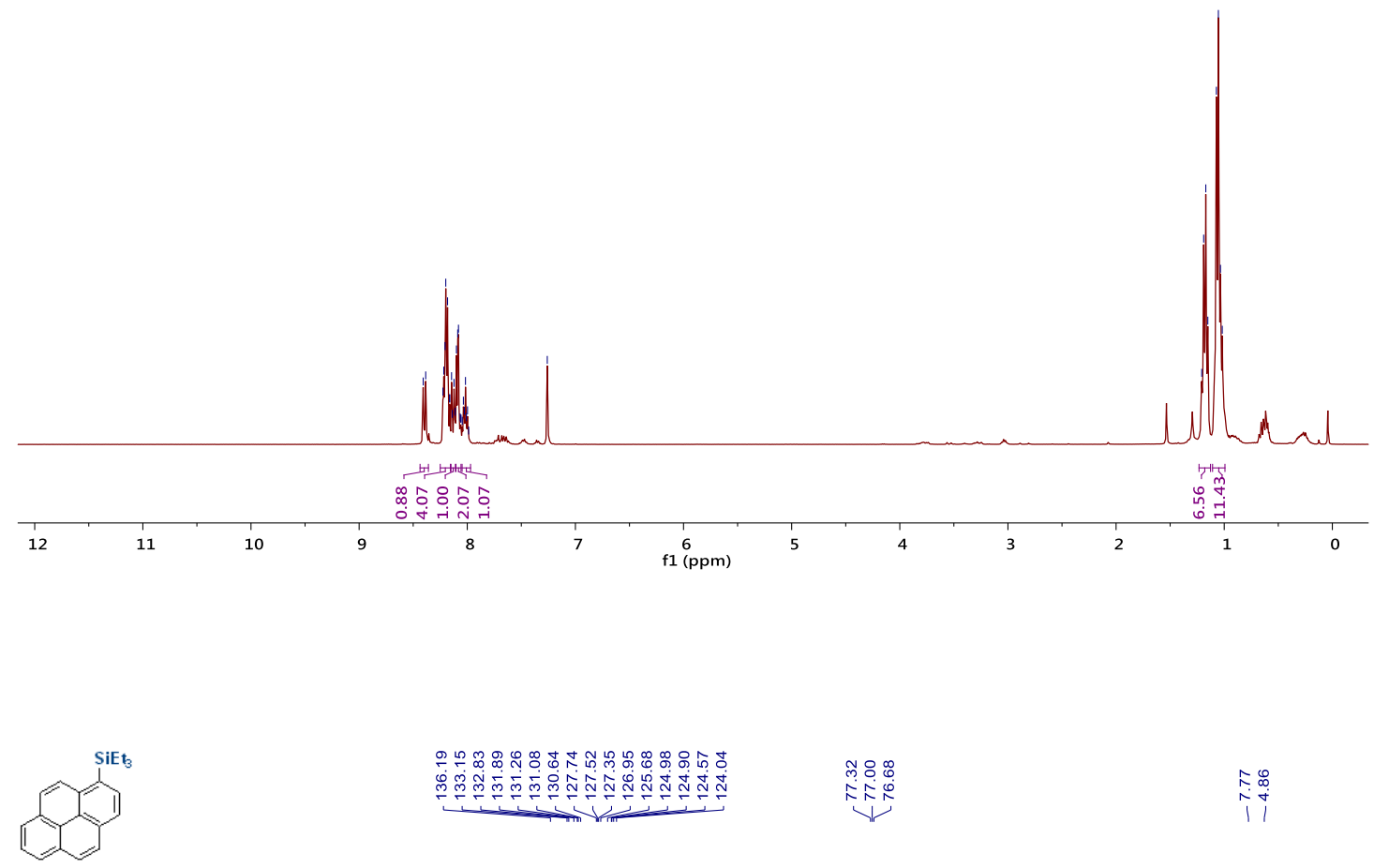

${ }^{13} \mathrm{C} \mathrm{NMR}\left(100 \mathrm{MHz}, \mathrm{CDCl}_{3}\right)$

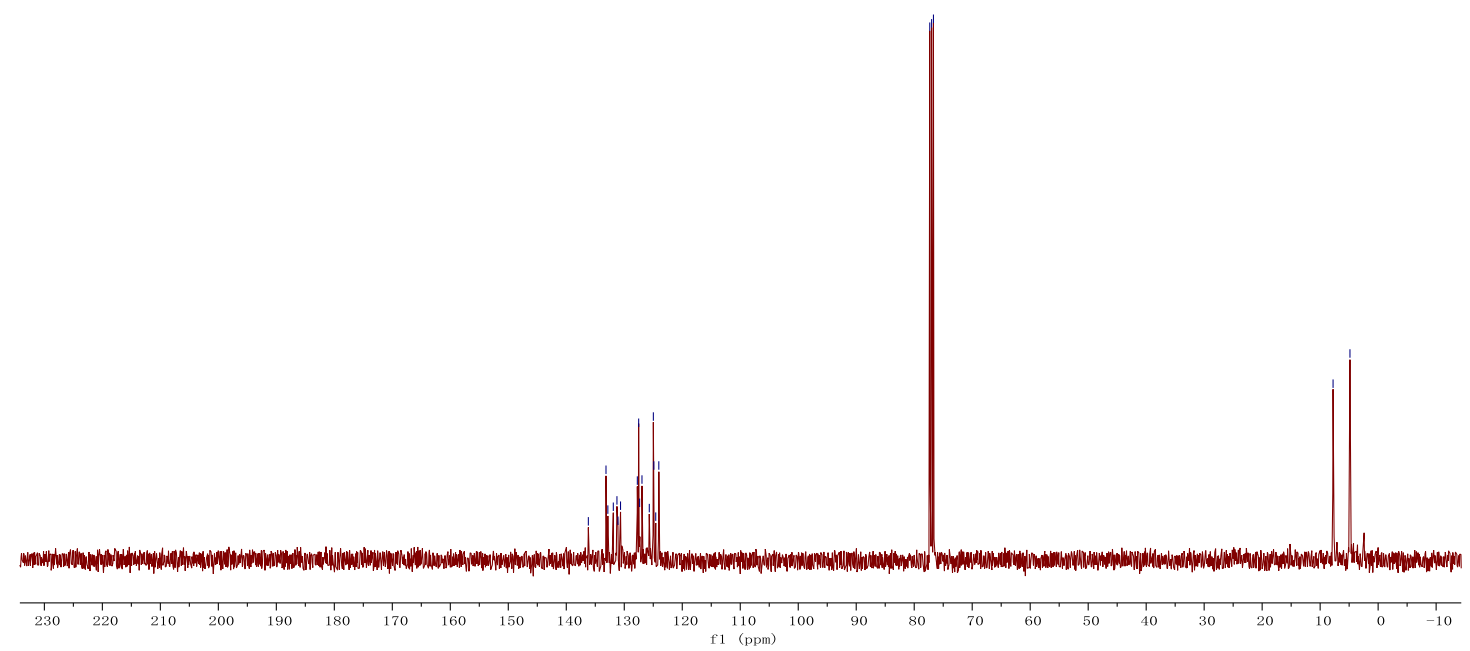




\section{0-(triethylsilyl)benzo[h]quinoline (15)}
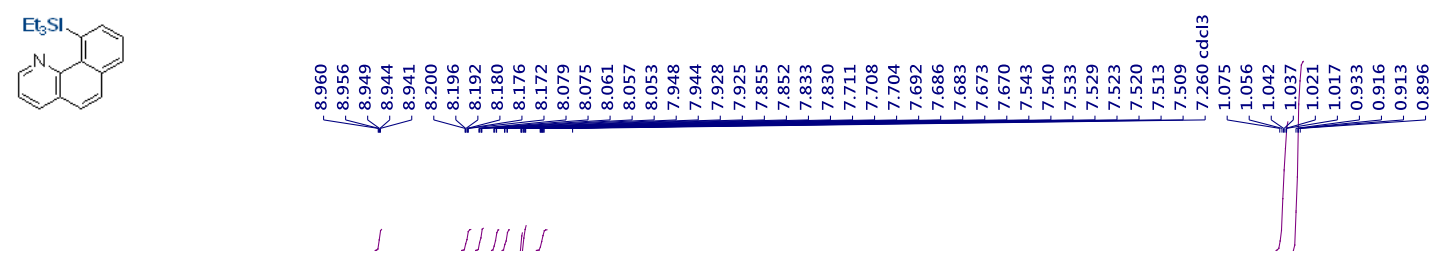

${ }^{1} \mathrm{H} \mathrm{NMR}\left(400 \mathrm{MHz}, \mathrm{CDCl}_{3}\right)$
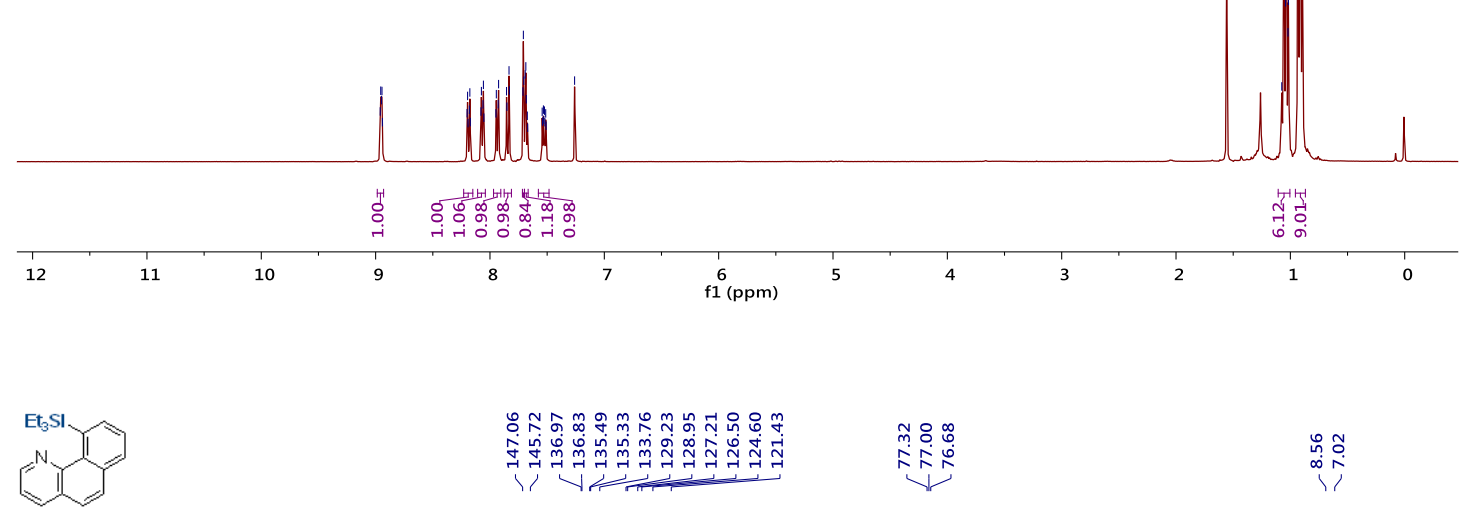

i.

${ }^{13} \mathrm{C}$ NMR $\left(100 \mathrm{MHz}, \mathrm{CDCl}_{3}\right)$ 
Triethyl(4'-methoxy-[1,1'-biphenyl]-4-yl)silane (16)
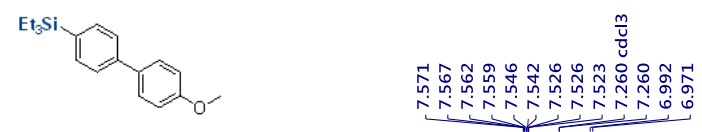

$\underset{\substack{n \\ \infty}}{\infty}$

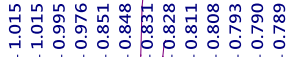

${ }^{1} \mathrm{H}$ NMR (400 MHz, $\mathrm{CDCl}_{3}$ )
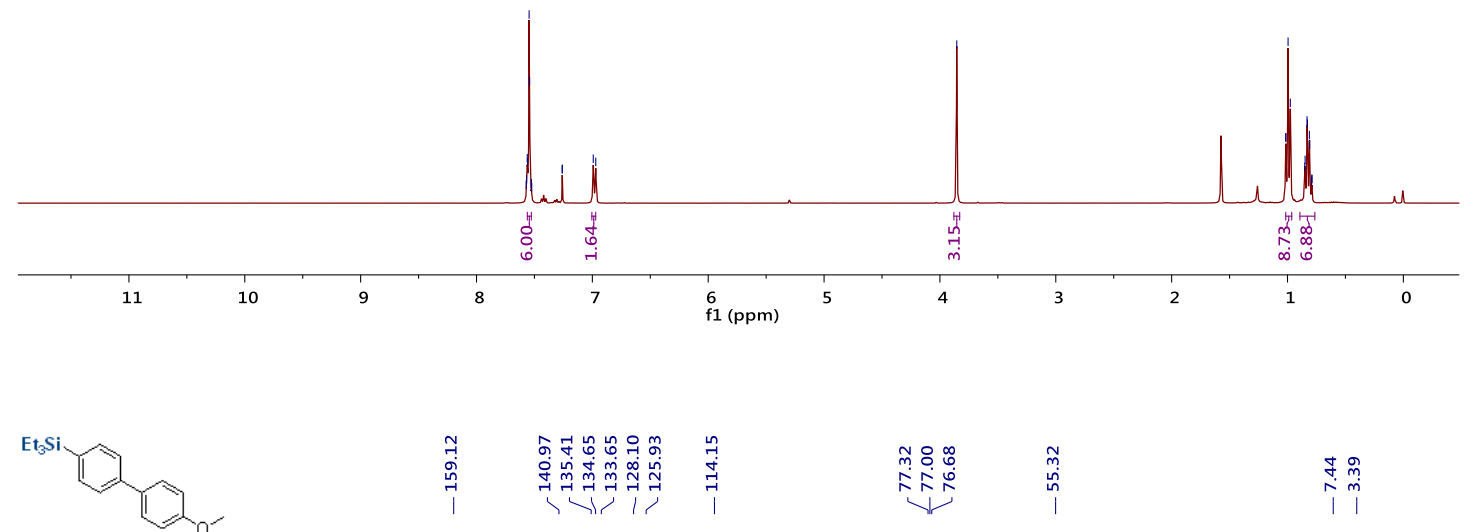

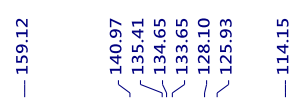

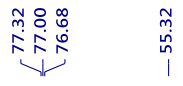

$\stackrel{\substack{* \\ i}}{\stackrel{m}{m}}$

${ }^{13} \mathrm{C} \mathrm{NMR}\left(100 \mathrm{MHz}, \mathrm{CDCl}_{3}\right)$

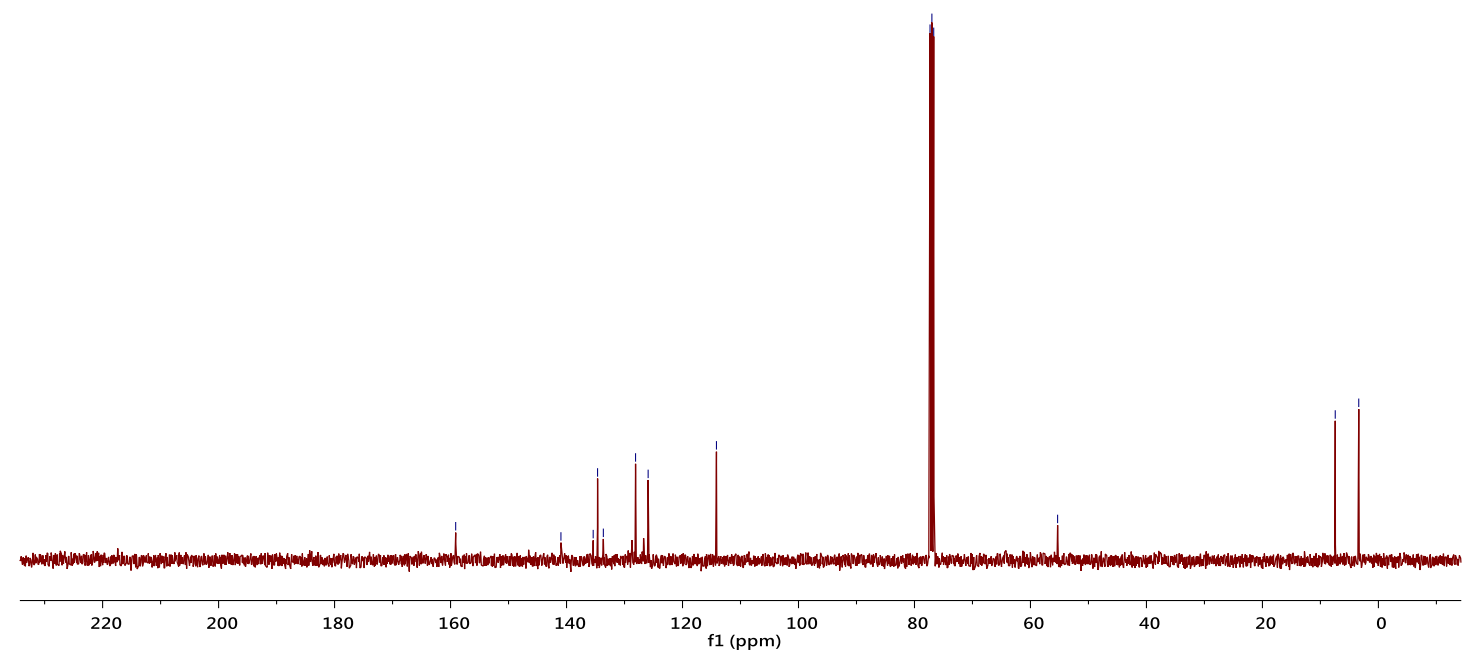


Triethyl(4-(naphthalen-1-yl)phenyl)silane (17)
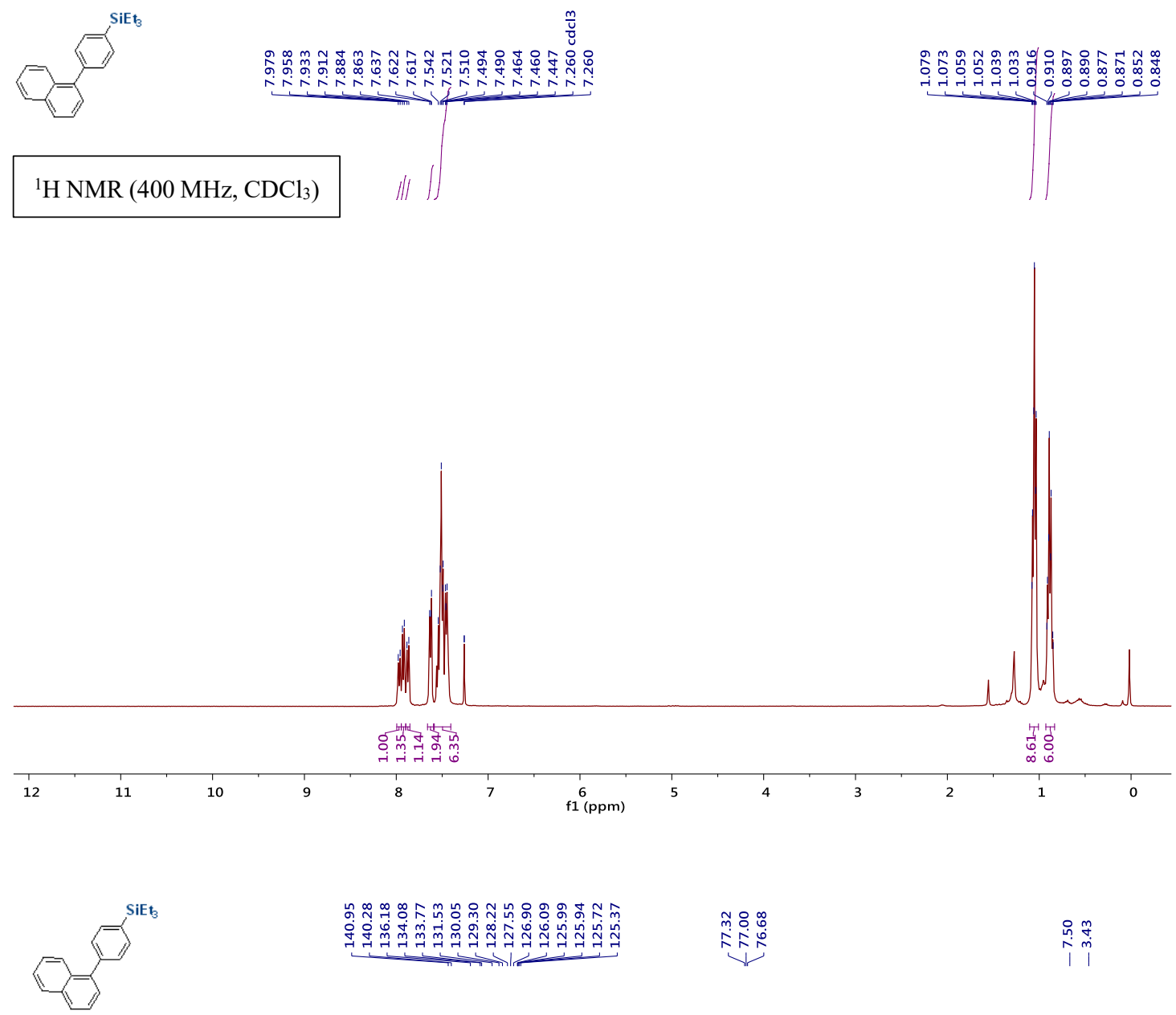

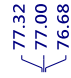

${ }^{13} \mathrm{C}$ NMR $\left(100 \mathrm{MHz}, \mathrm{CDCl}_{3}\right)$

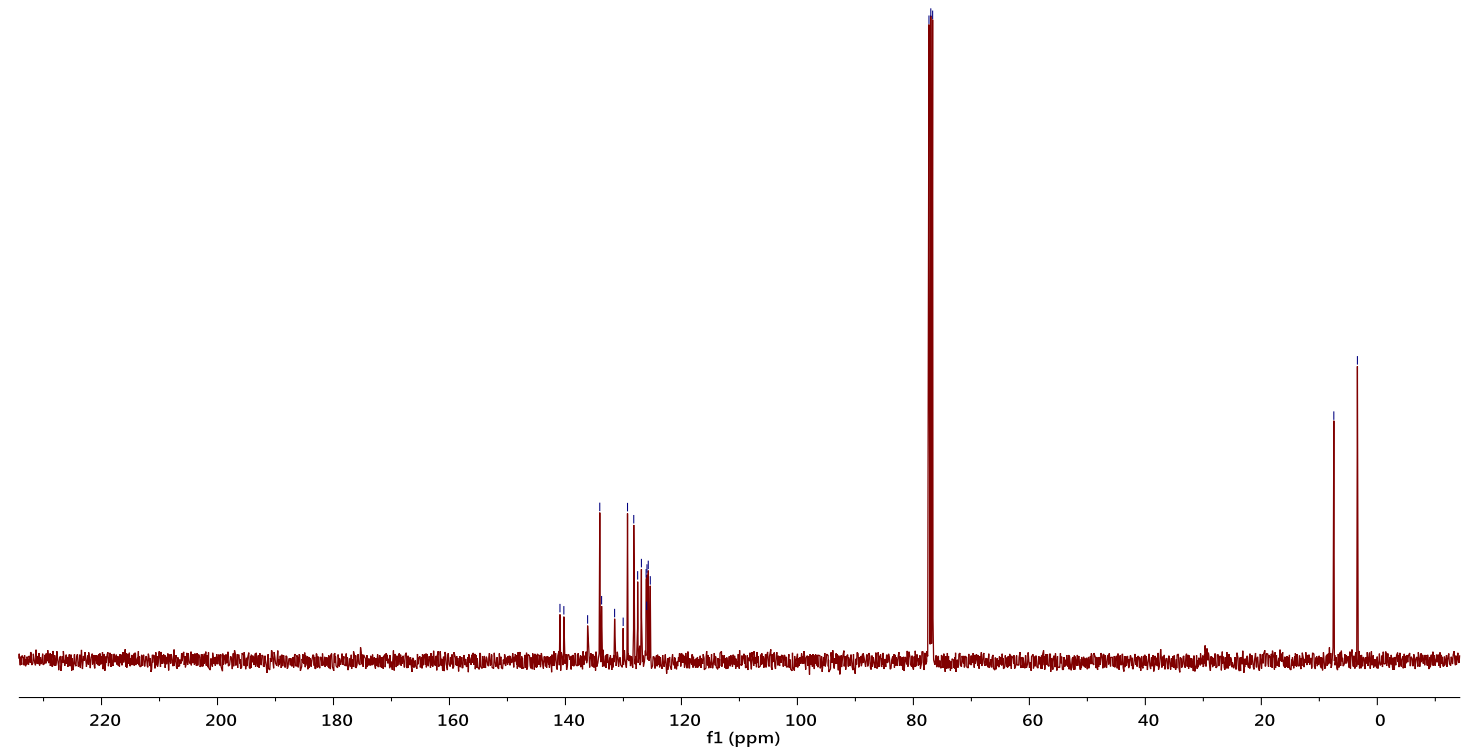


Triethyl(4-((triisopropylsilyl)ethynyl)phenyl)silane (18)
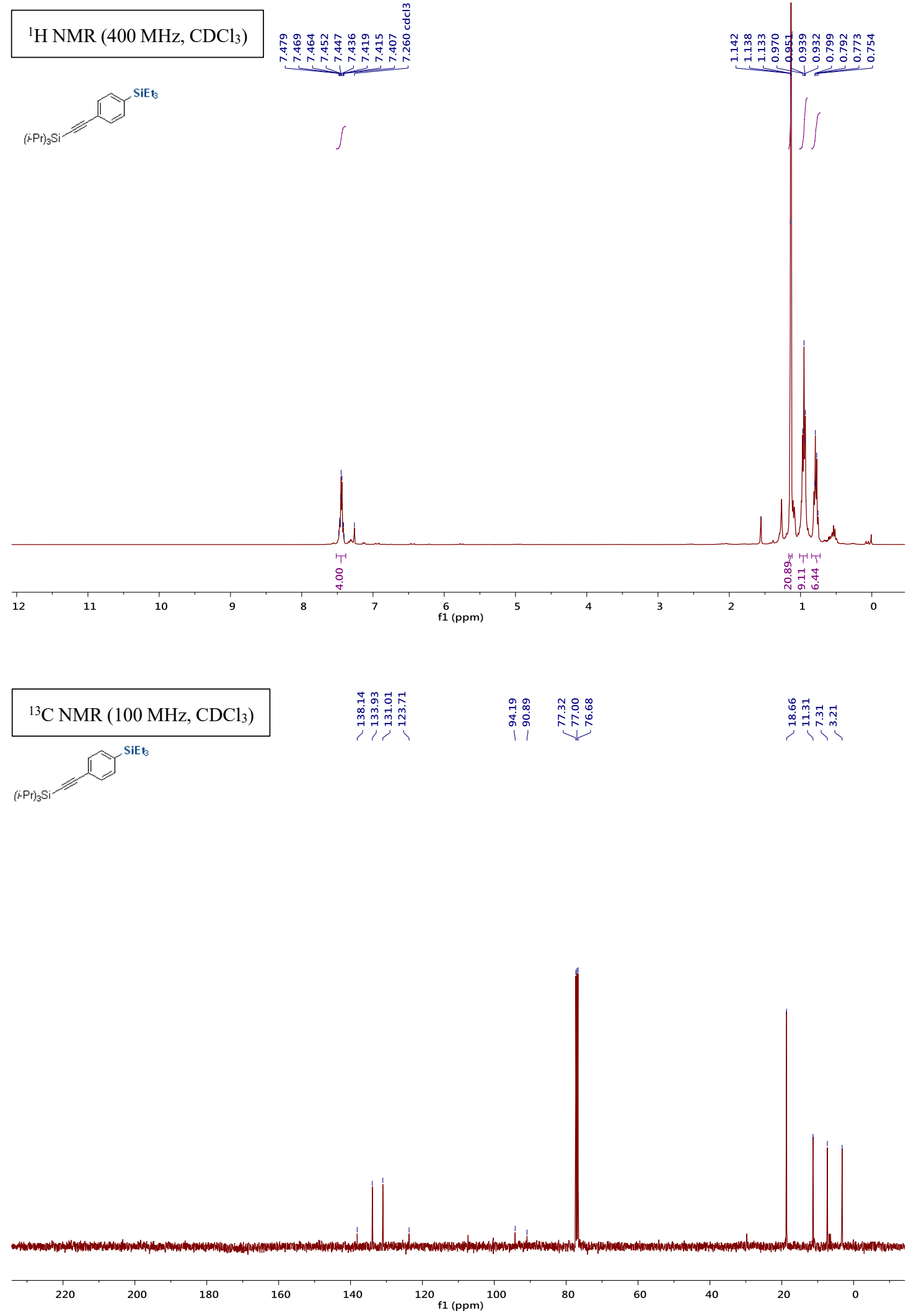
(4-(tert-Butyl)phenyl)triethylsilane (19)
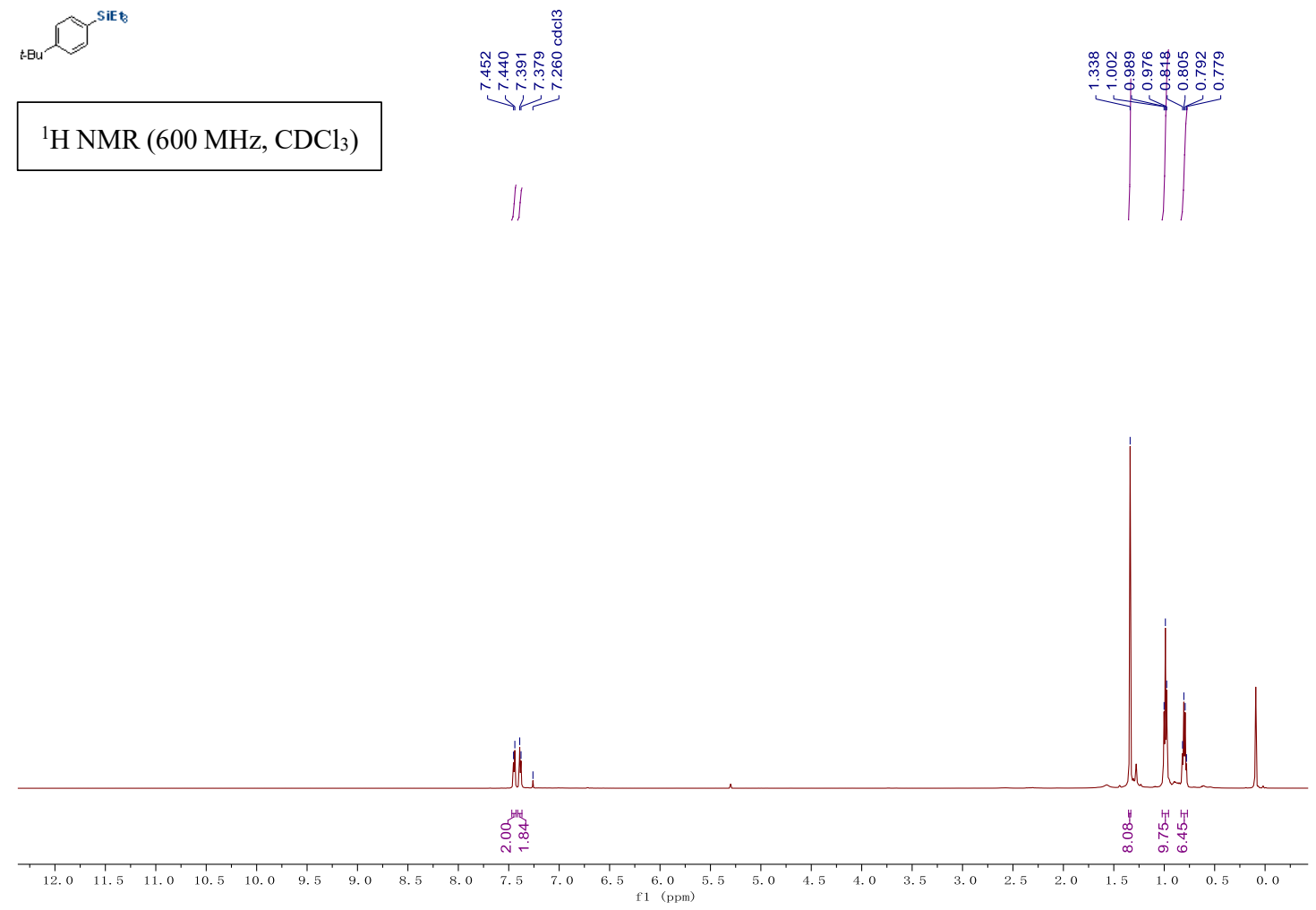

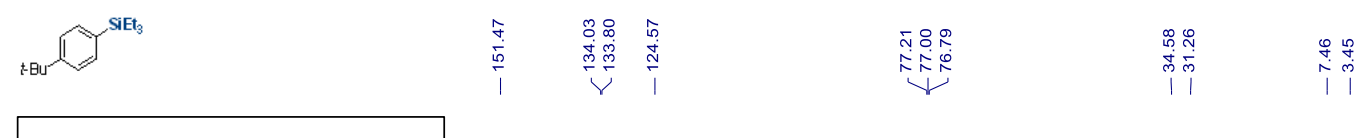

${ }^{13} \mathrm{C}$ NMR $\left(125 \mathrm{MHz}, \mathrm{CDCl}_{3}\right)$

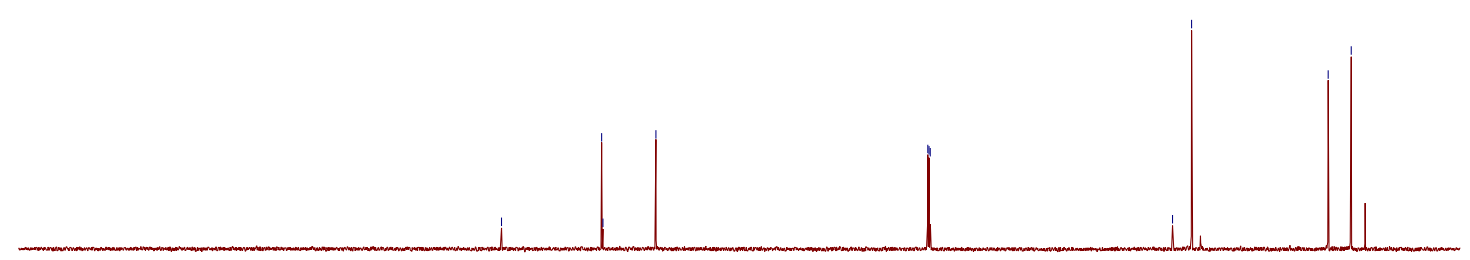

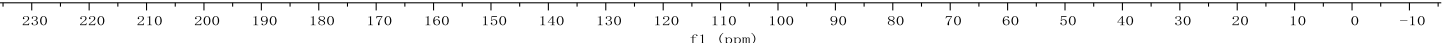


(4-Cyclohexylphenyl)triethylsilane (20)
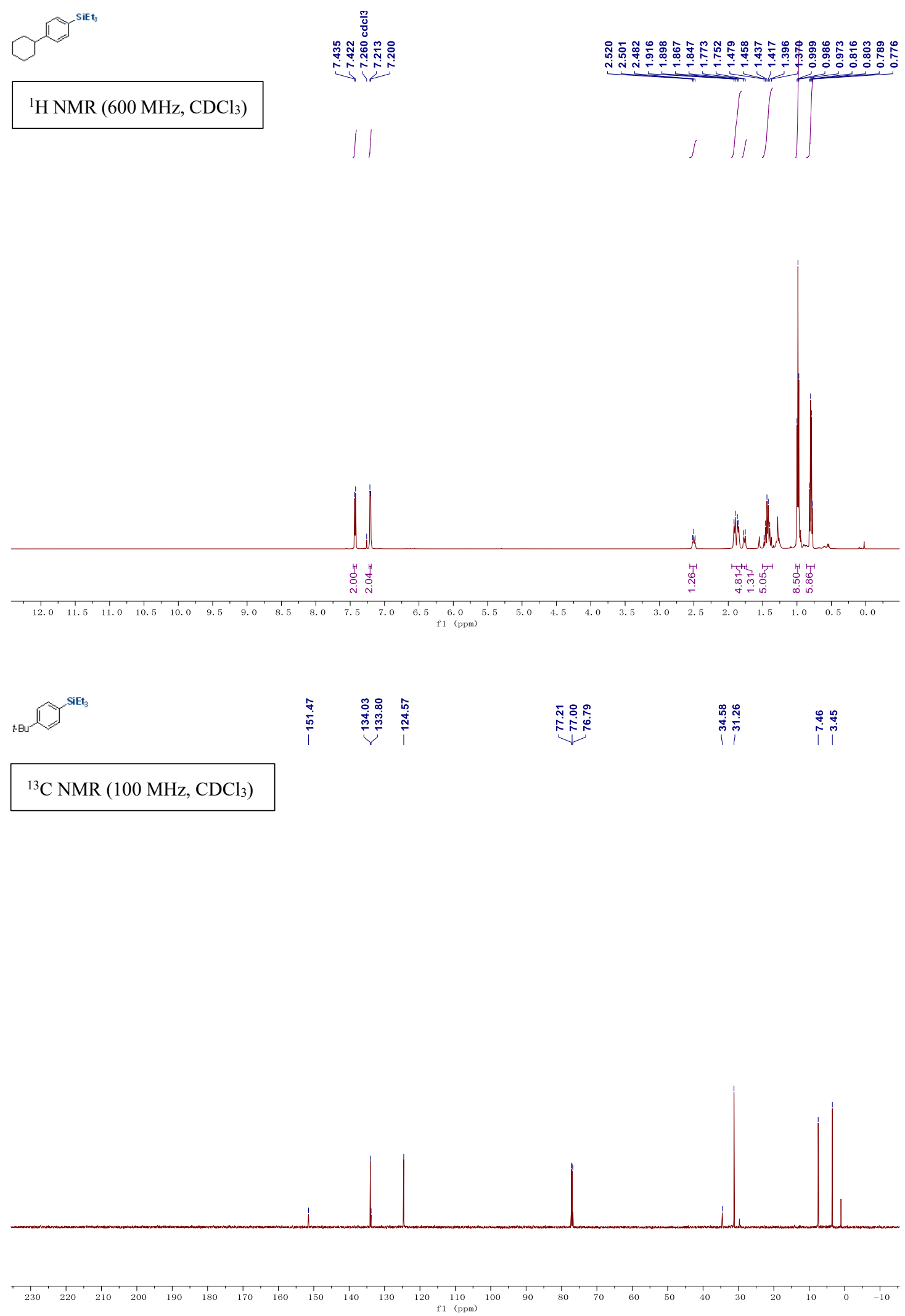
(3,5-Dimethylphenyl)triethylsilane (21)
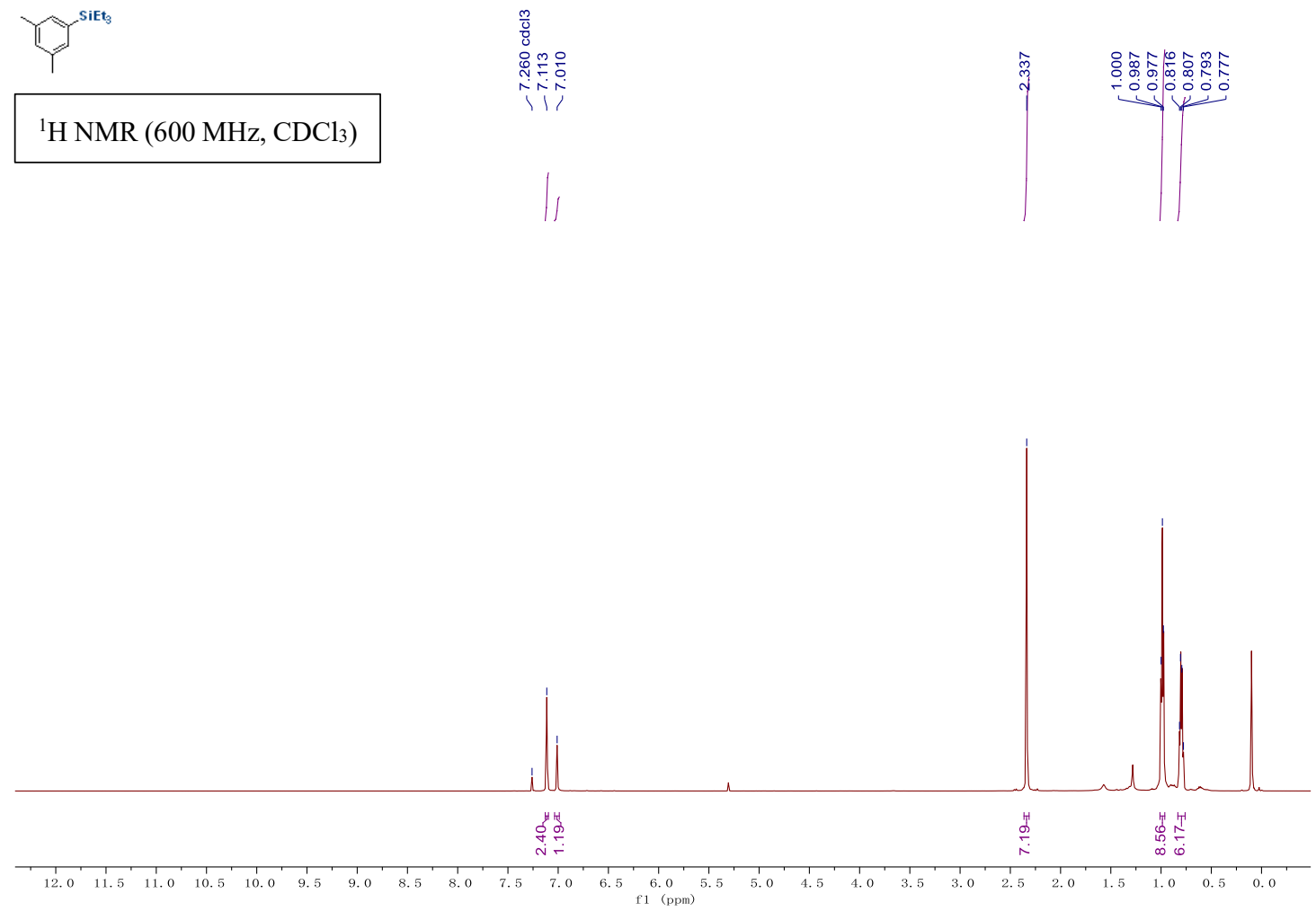

$y^{\text {siets }}$

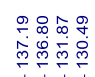

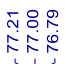

$\stackrel{\substack{1 \\ 7}}{\stackrel{1}{7}}$

${ }^{13} \mathrm{C}$ NMR (125 MHz, $\left.\mathrm{CDCl}_{3}\right)$
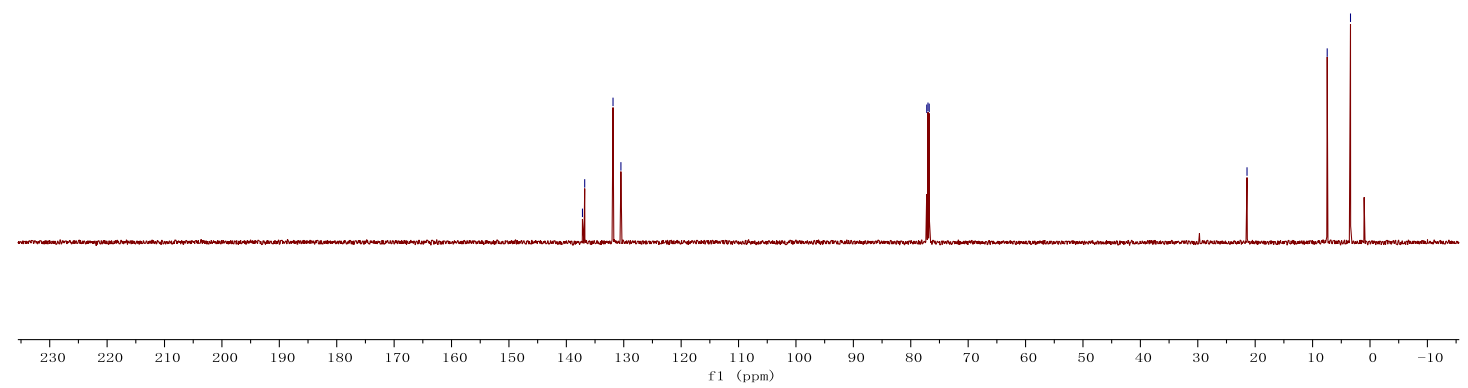
Triethyl(5,6,7,8-tetrahydronaphthalen-2-yl)silane (22).

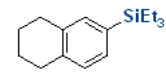

${ }^{1} \mathrm{H} \mathrm{NMR}\left(600 \mathrm{MHz}, \mathrm{CDCl}_{3}\right)$
응

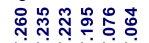

年

III

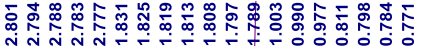

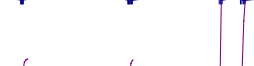

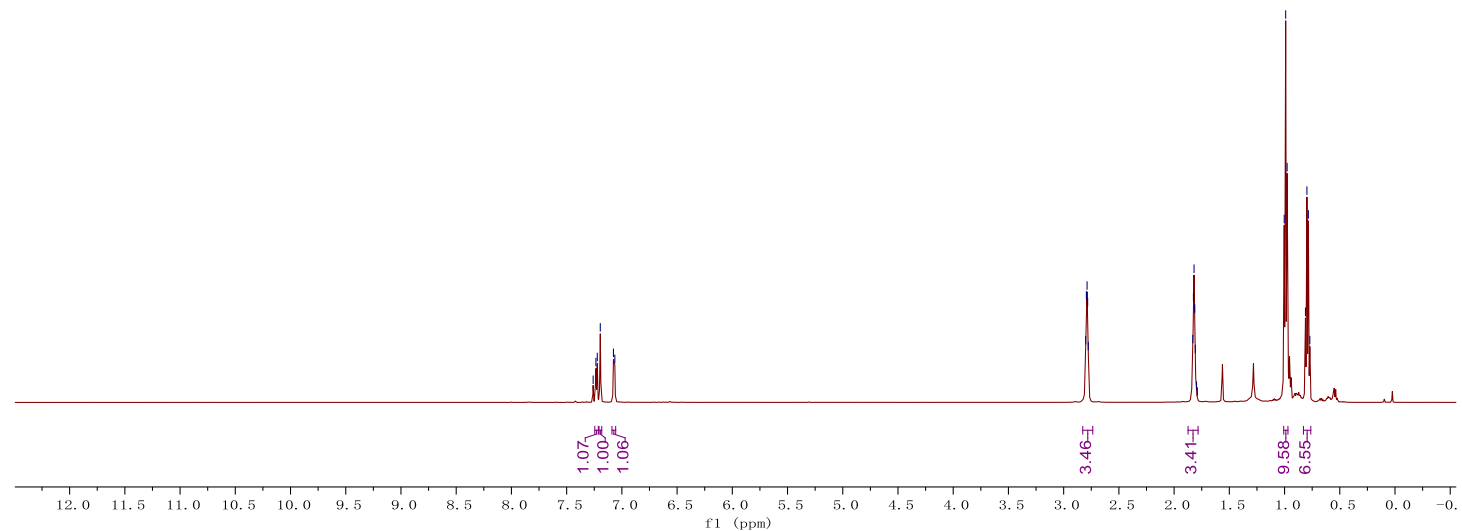

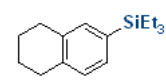

${ }^{13} \mathrm{C}$ NMR $\left(100 \mathrm{MHz}, \mathrm{CDCl}_{3}\right)$

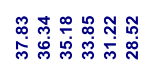

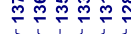

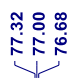

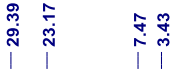

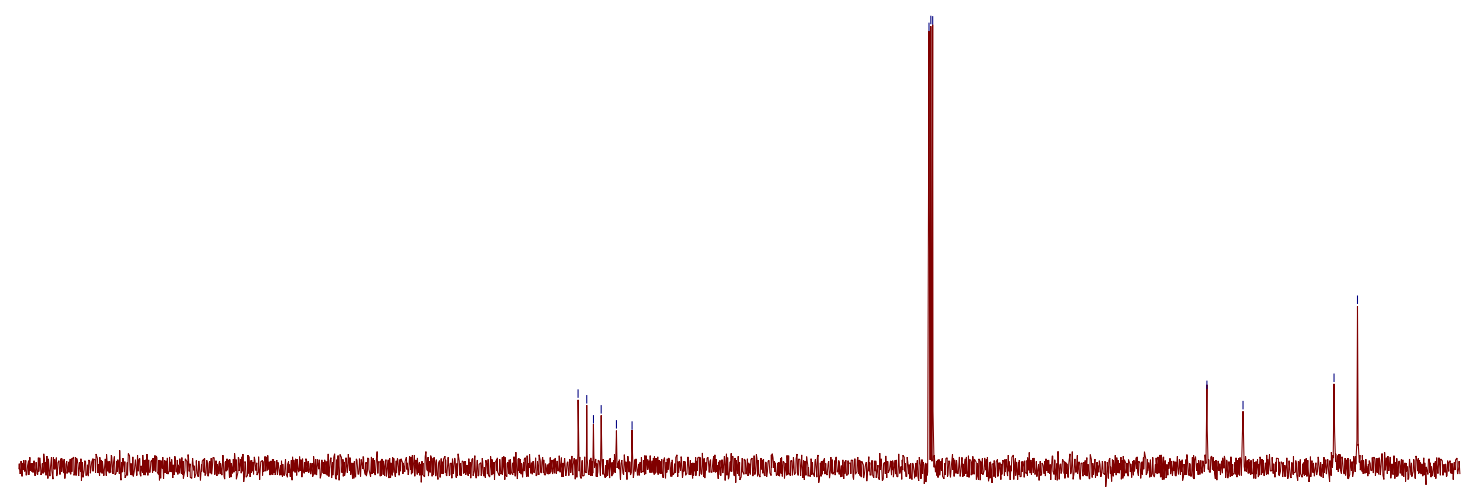

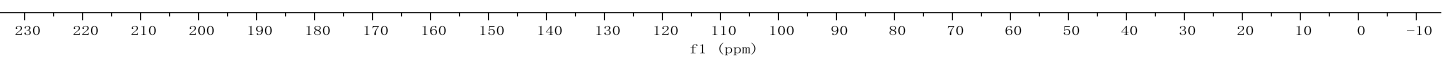


Triethyl(4-isopropylphenyl)silane (23).
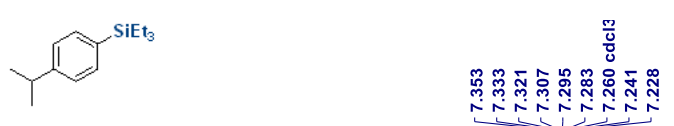

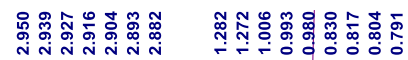

${ }^{1} \mathrm{H}$ NMR $\left(400 \mathrm{MHz}, \mathrm{CDCl}_{3}\right)$
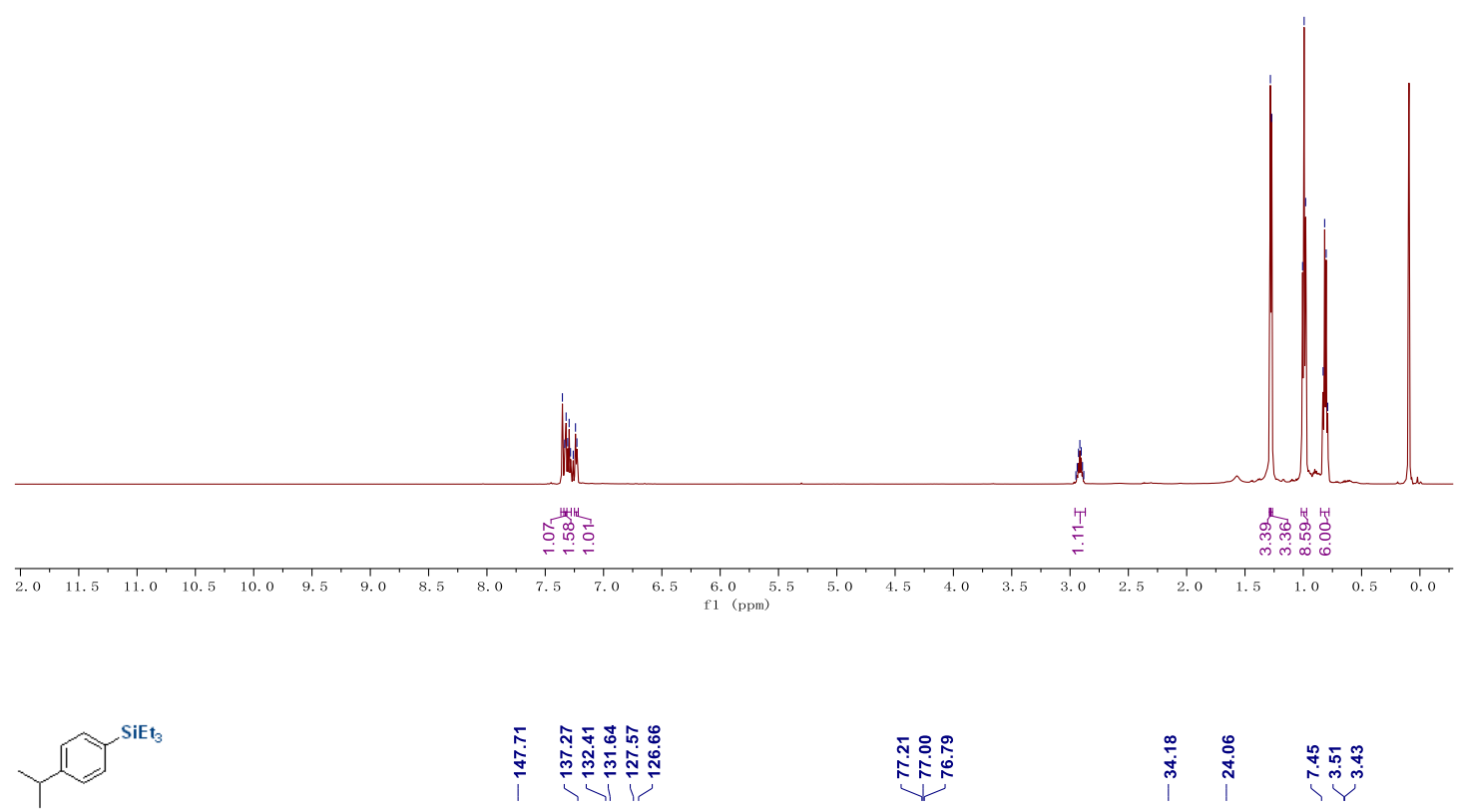

$\underbrace{\sqrt{n+1}}$

势

${ }^{13} \mathrm{C}$ NMR (125 MHz, $\left.\mathrm{CDCl}_{3}\right)$
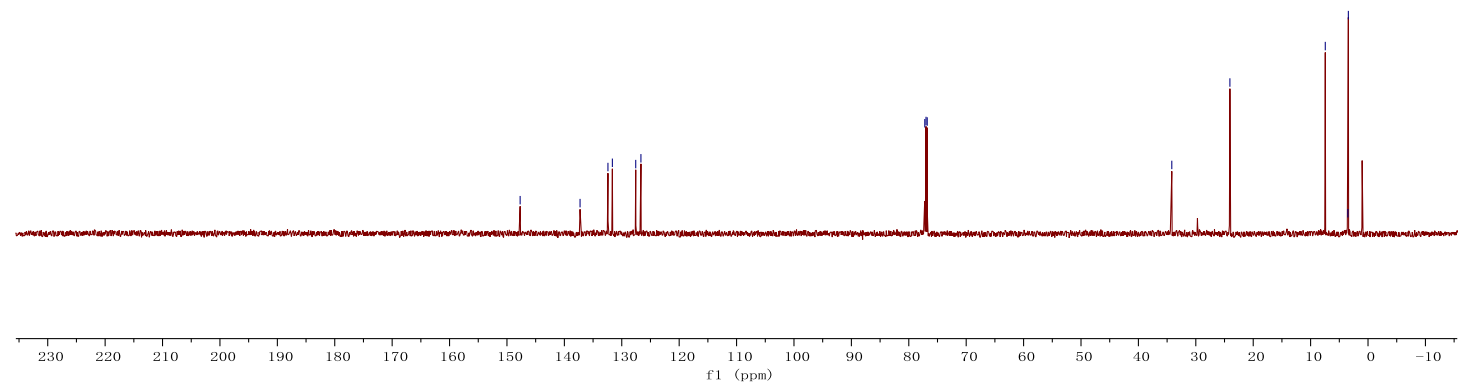
Triethyl(4-(2-phenylpropan-2-yl)phenyl)silane (24)
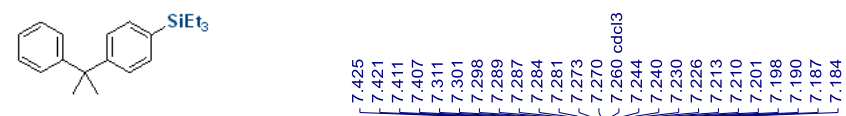

${ }^{1} \mathrm{H} \mathrm{NMR}\left(600 \mathrm{MHz}, \mathrm{CDCl}_{3}\right)$

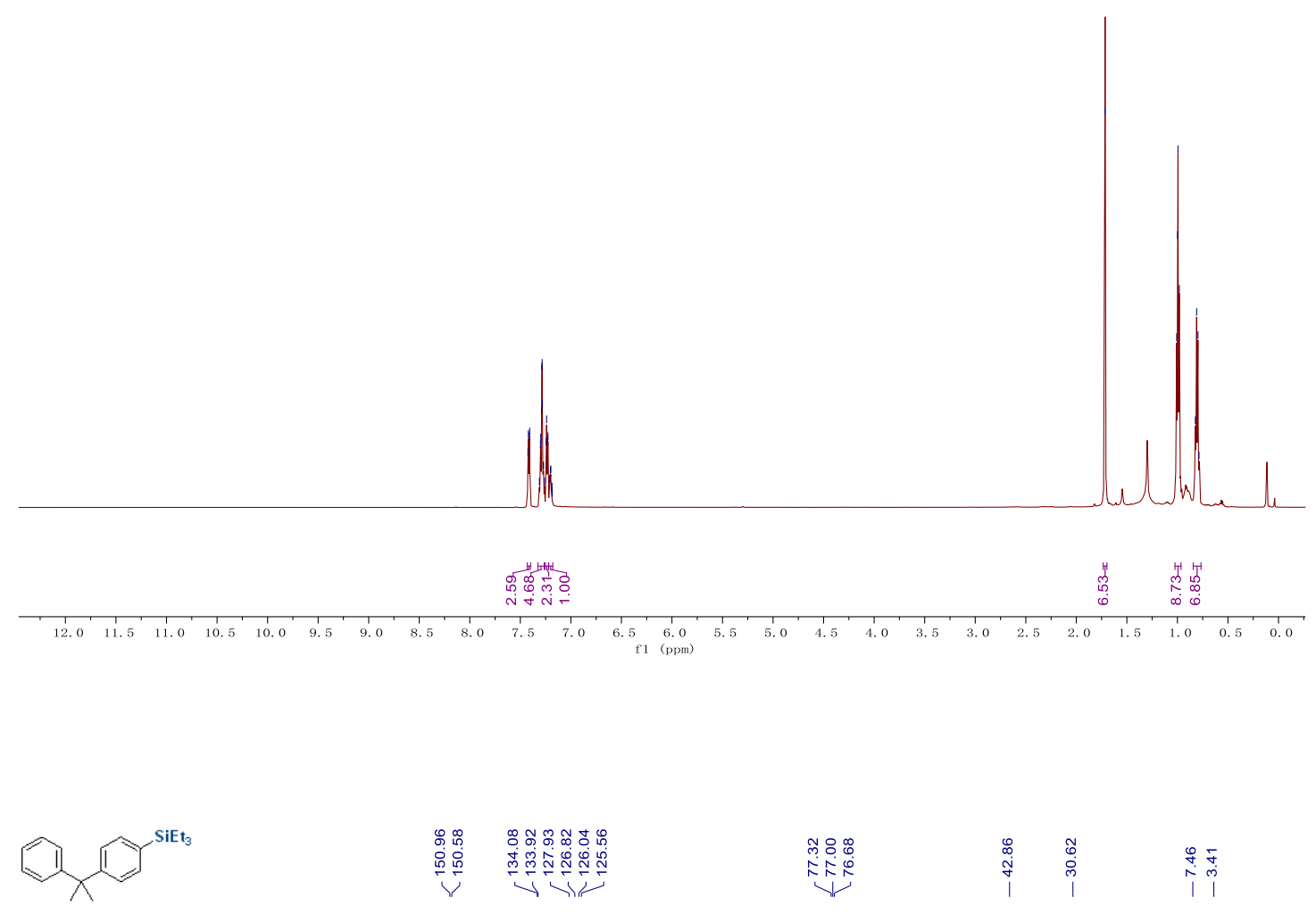

${ }^{13} \mathrm{C}$ NMR $\left(100 \mathrm{MHz}, \mathrm{CDCl}_{3}\right)$

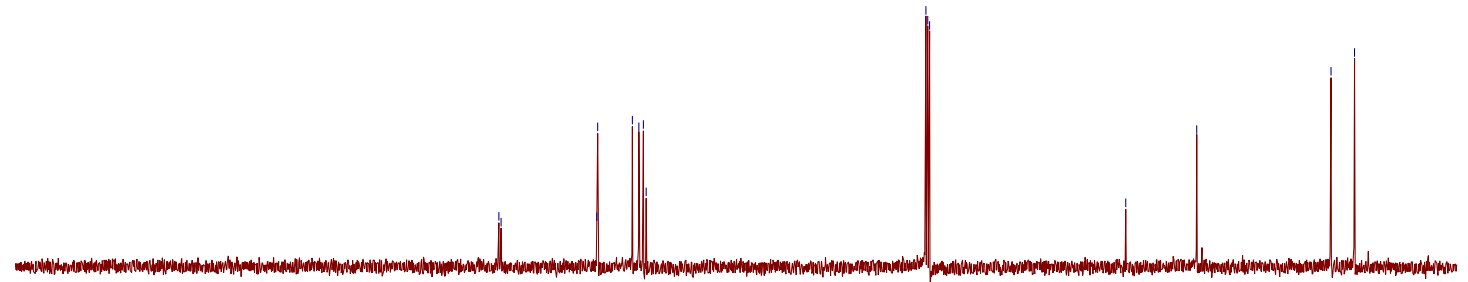

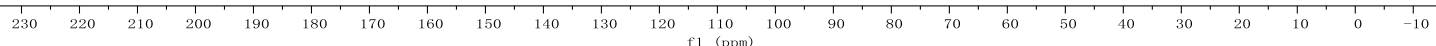


(4-(4-Butylcyclohexyl)phenyl)triethylsilane (25)

营

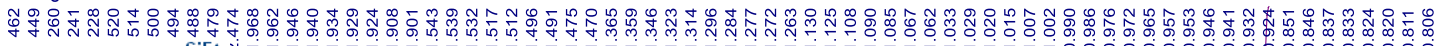

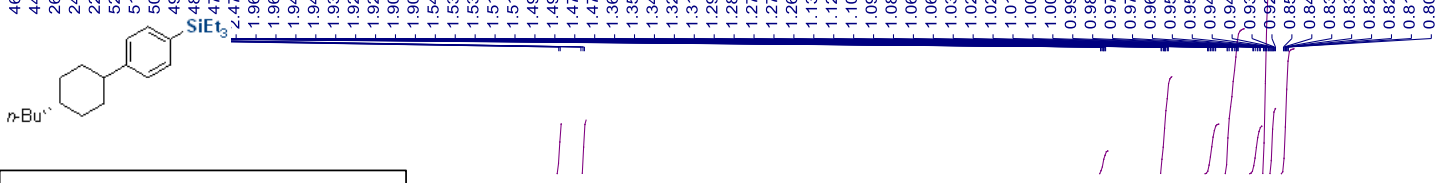

${ }^{1} \mathrm{H} \mathrm{NMR}\left(600 \mathrm{MHz}, \mathrm{CDCl}_{3}\right)$
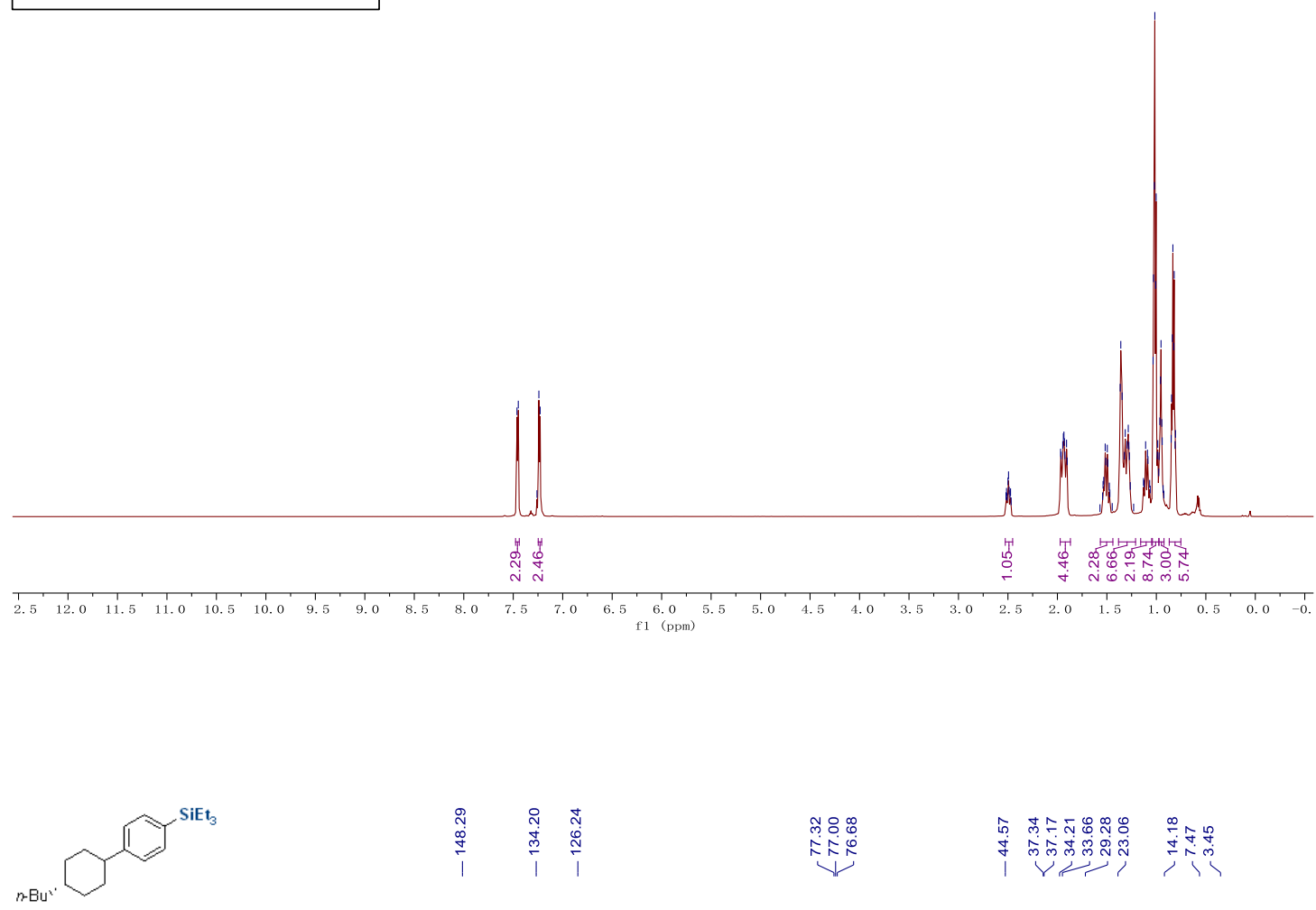

I

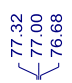

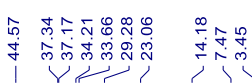

${ }^{13} \mathrm{C} \mathrm{NMR}\left(100 \mathrm{MHz}, \mathrm{CDCl}_{3}\right)$

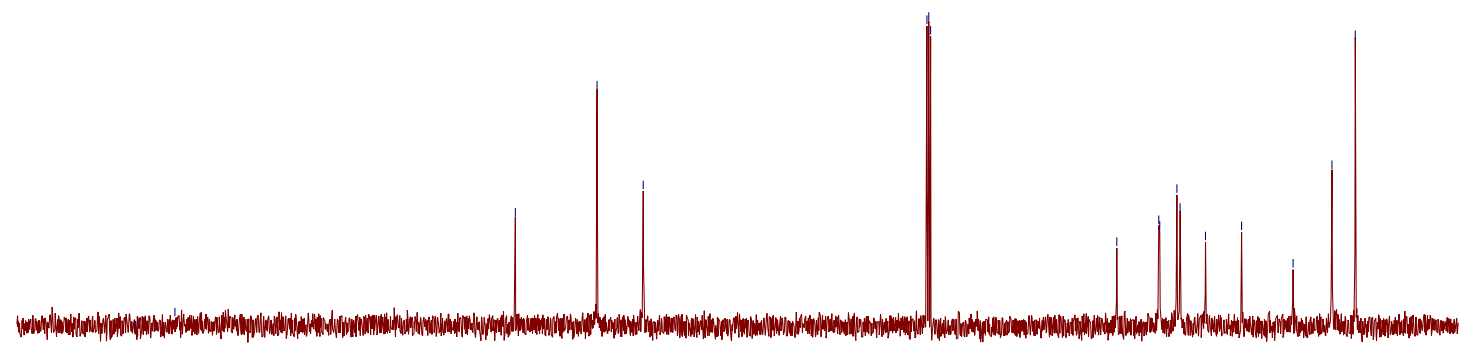

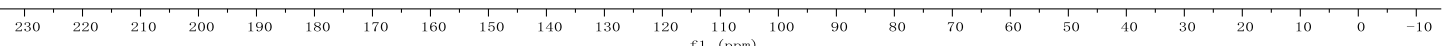


Triethyl(p-tolyl)silane (26).
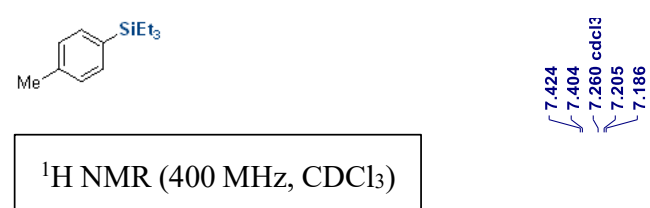

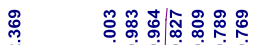

补

11

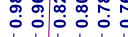

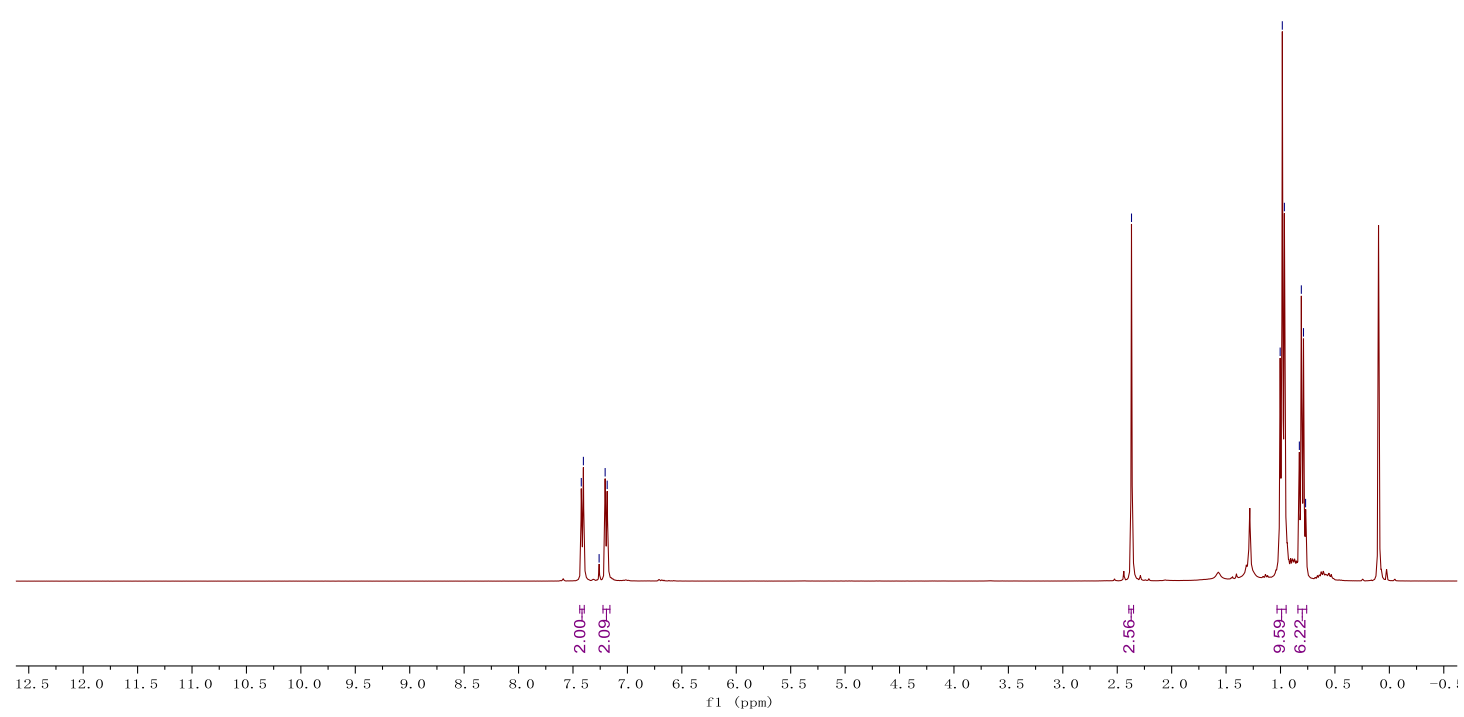

$\mathrm{SiEt}_{3}$

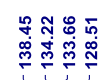

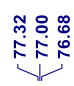

i

${ }^{13} \mathrm{C} \mathrm{NMR}\left(100 \mathrm{MHz}, \mathrm{CDCl}_{3}\right)$

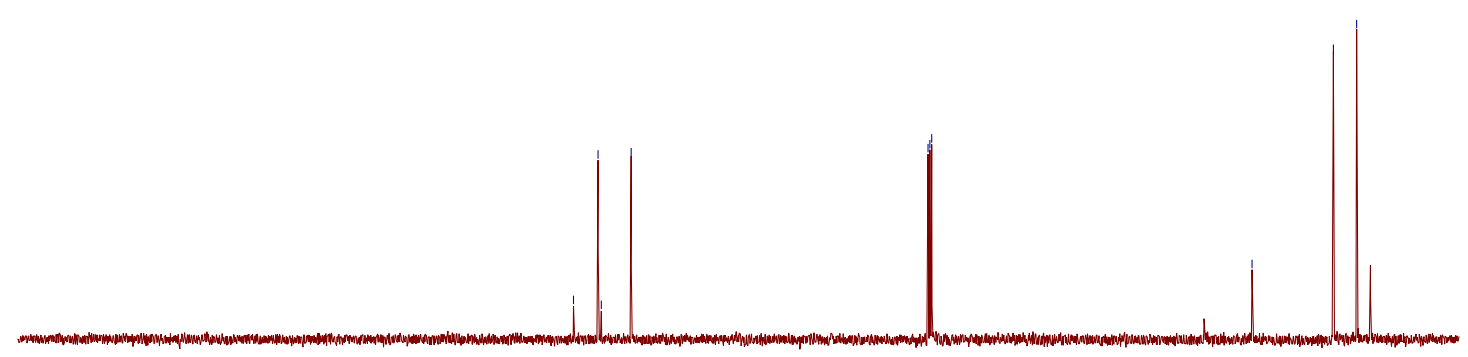

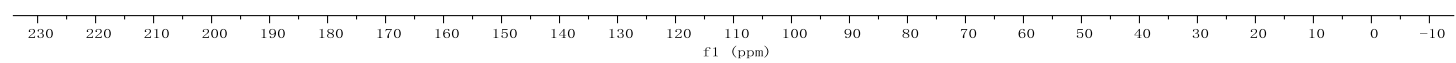


Triethyl(4-(methylthio)phenyl)silane (27)
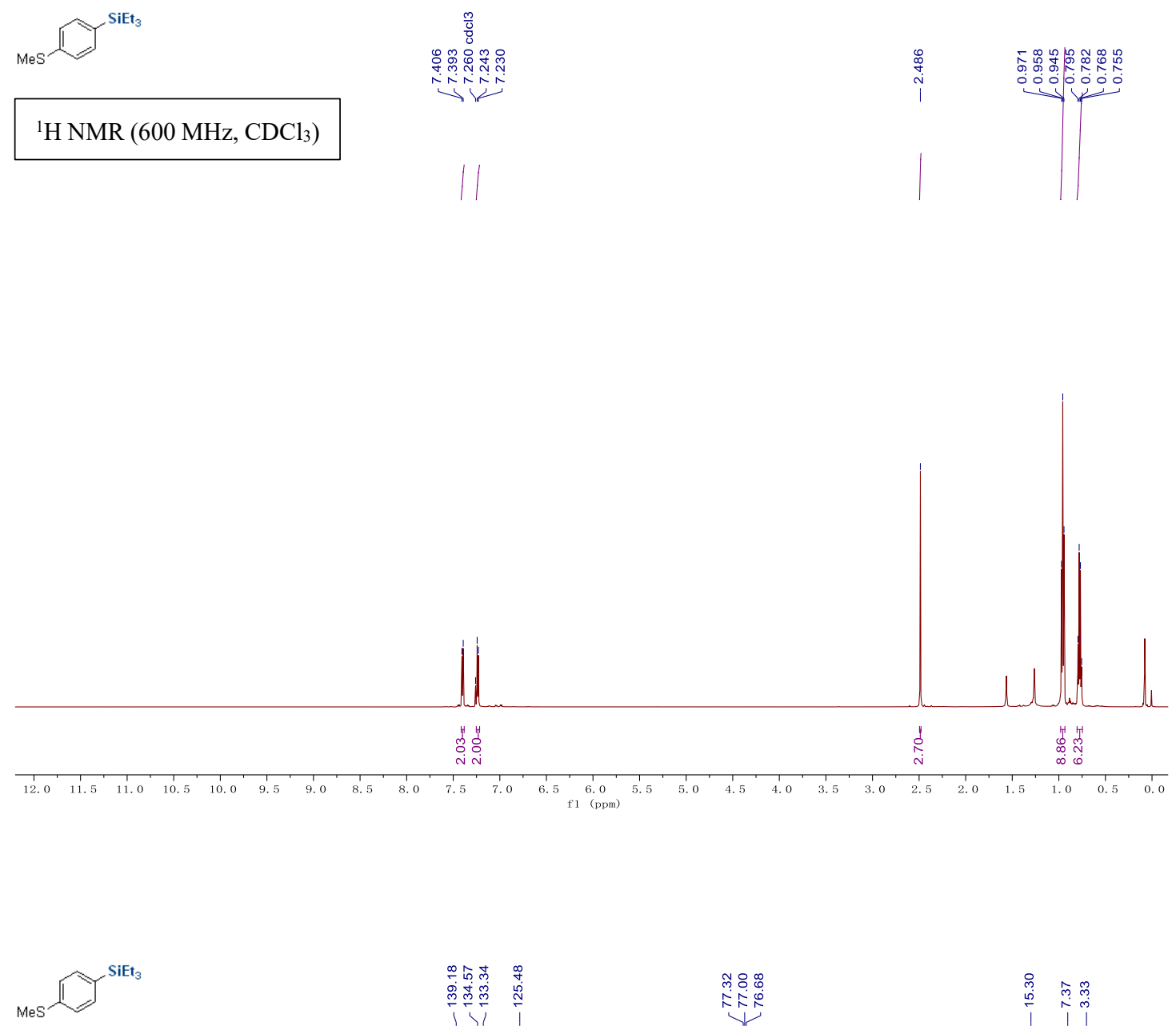

年

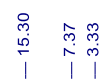

${ }^{13} \mathrm{C} \mathrm{NMR}\left(100 \mathrm{MHz}, \mathrm{CDCl}_{3}\right)$

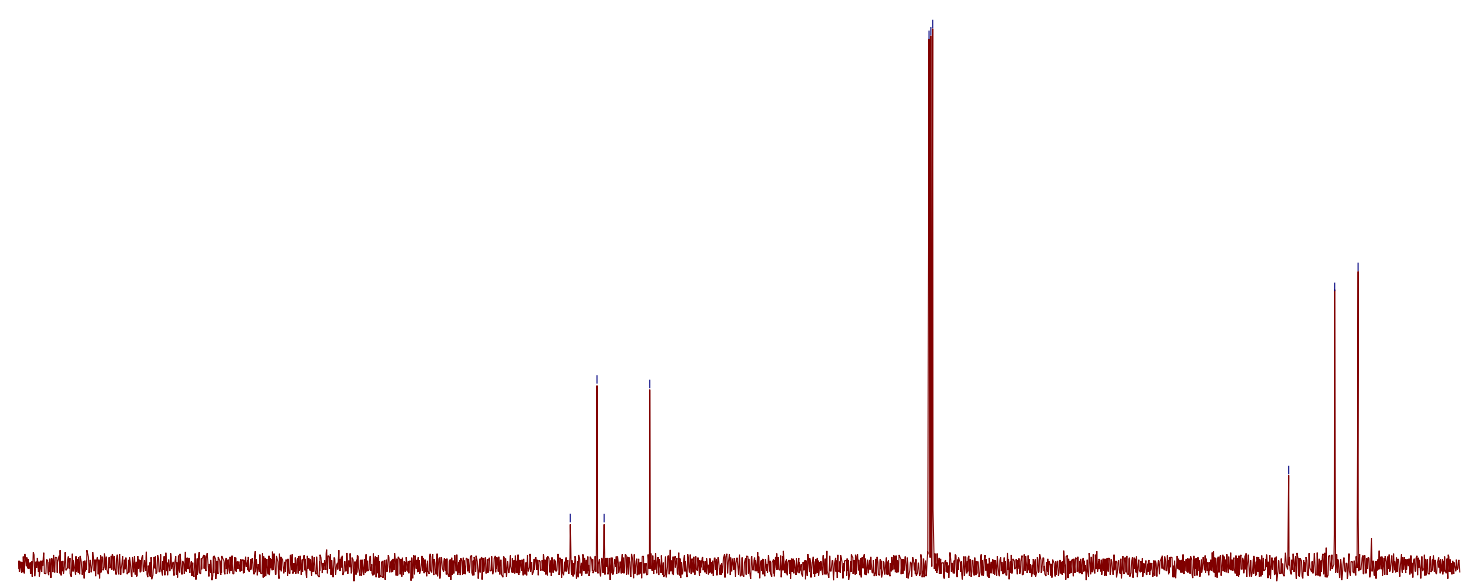

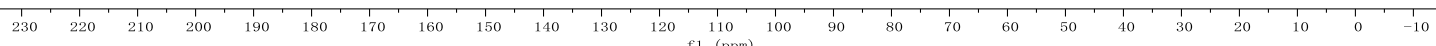


Triethyl[4-[(4-Methoxyphenyl)thio]phenyl]silane (28)

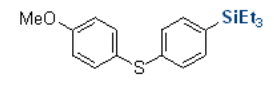

${ }^{1} \mathrm{H} \mathrm{NMR}\left(400 \mathrm{MHz}, \mathrm{CDCl}_{3}\right)$ $\frac{6}{8}$

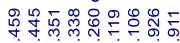

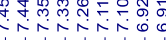

$\| 11$
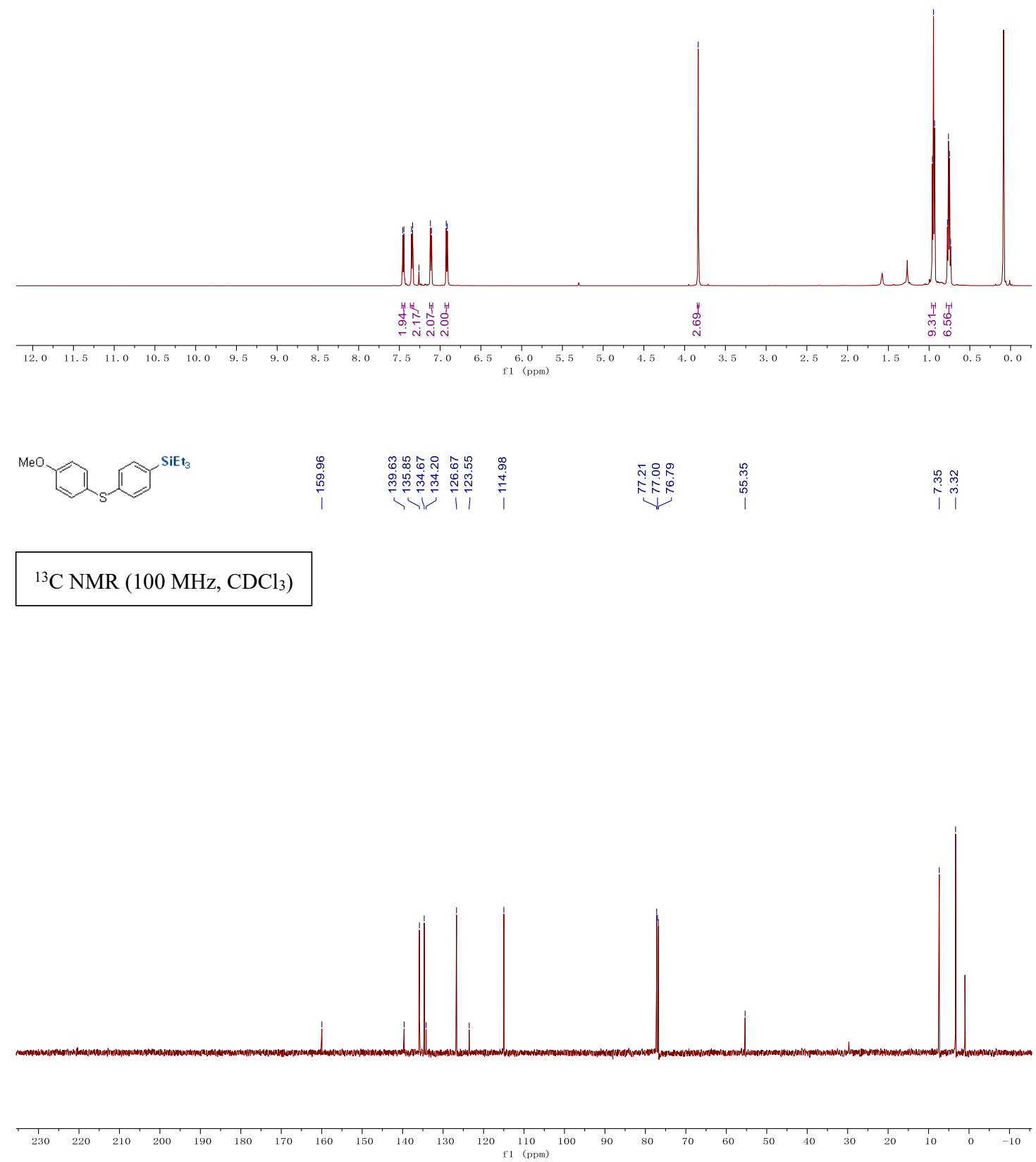
Benzo $[d][1,3]$ dioxol-5-yltriethylsilane (29)

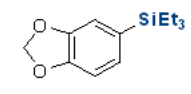

${ }^{1} \mathrm{H} \mathrm{NMR}\left(600 \mathrm{MHz}, \mathrm{CDCl}_{3}\right)$

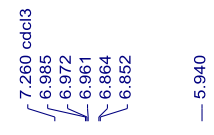

$\| 1$

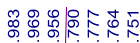

o. o. 00
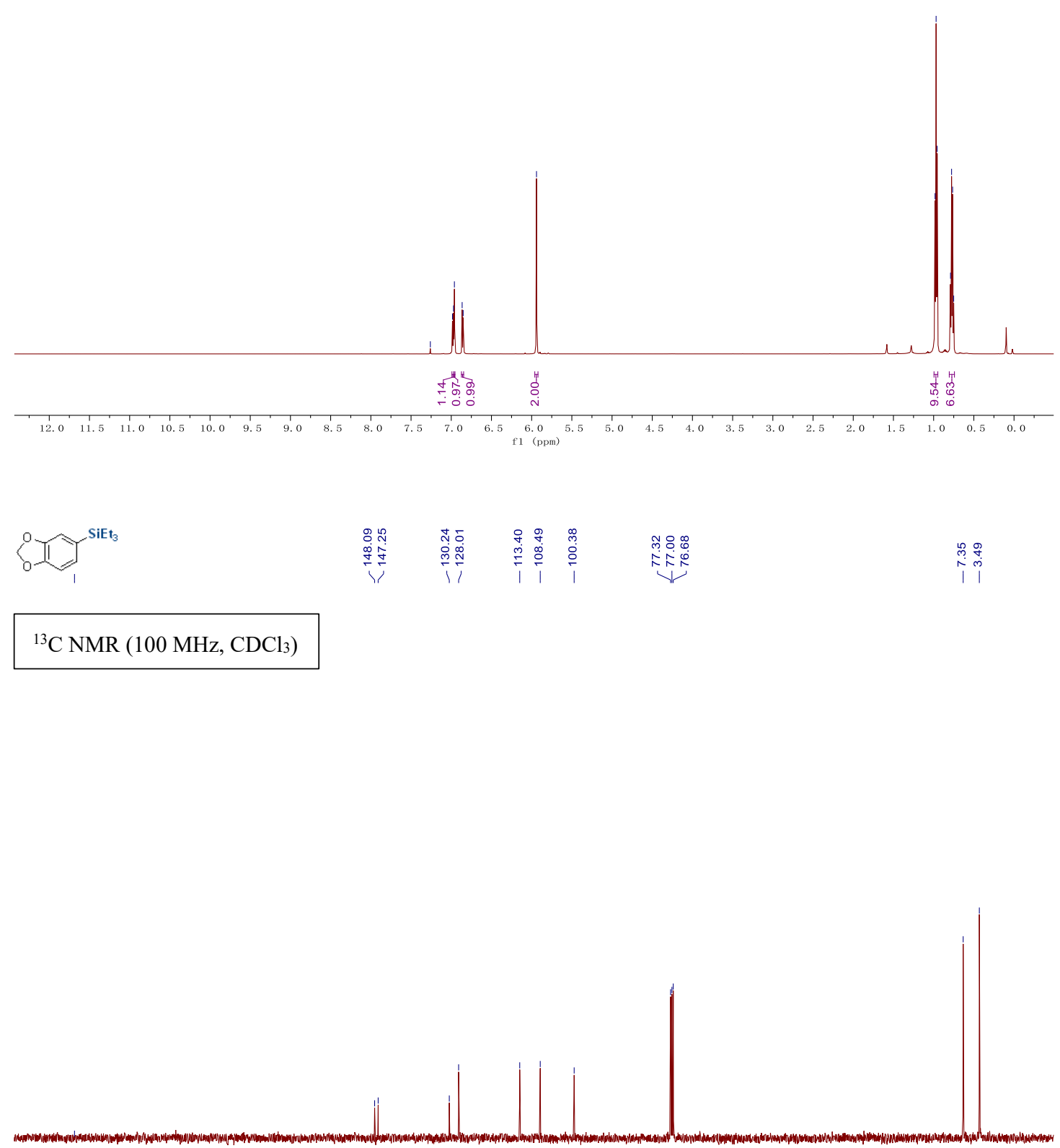

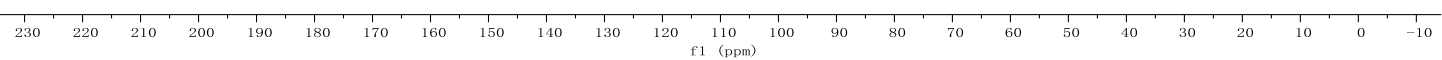


Triethyl(4-methoxyphenyl)silane (30)

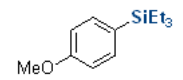

${ }^{1} \mathrm{H} \mathrm{NMR}\left(600 \mathrm{MHz}, \mathrm{CDCl}_{3}\right)$

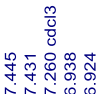

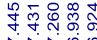

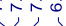

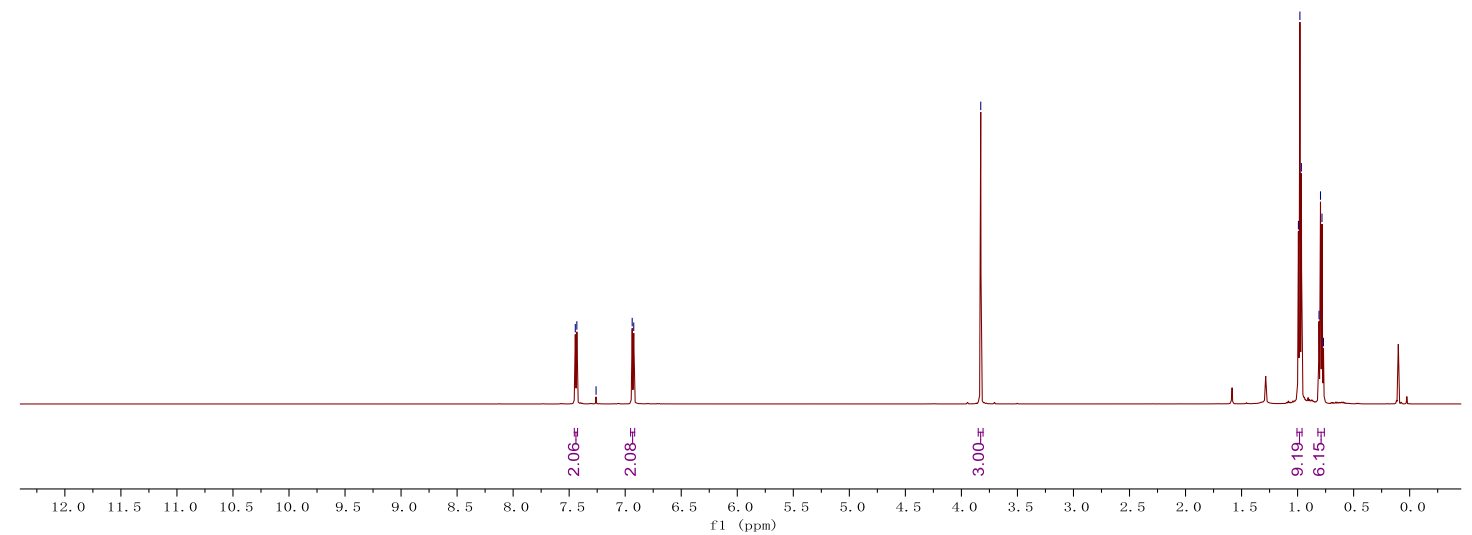

$\mathrm{N}^{\mathrm{SiEt}_{3}}$

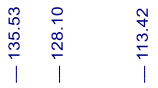

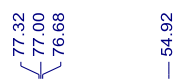

$\stackrel{\substack{j \\ i}}{\substack{g \\ m}}$

${ }^{13} \mathrm{C} \mathrm{NMR}\left(100 \mathrm{MHz}, \mathrm{CDCl}_{3}\right)$

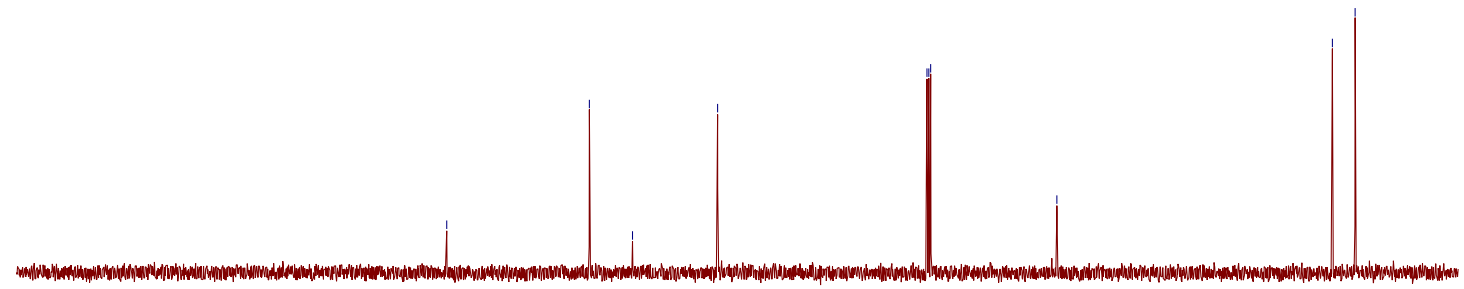

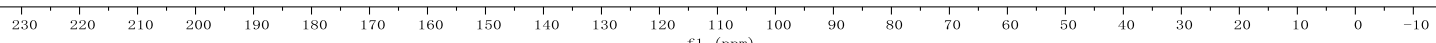


Triethyl(4-phenoxyphenyl)silane (31)
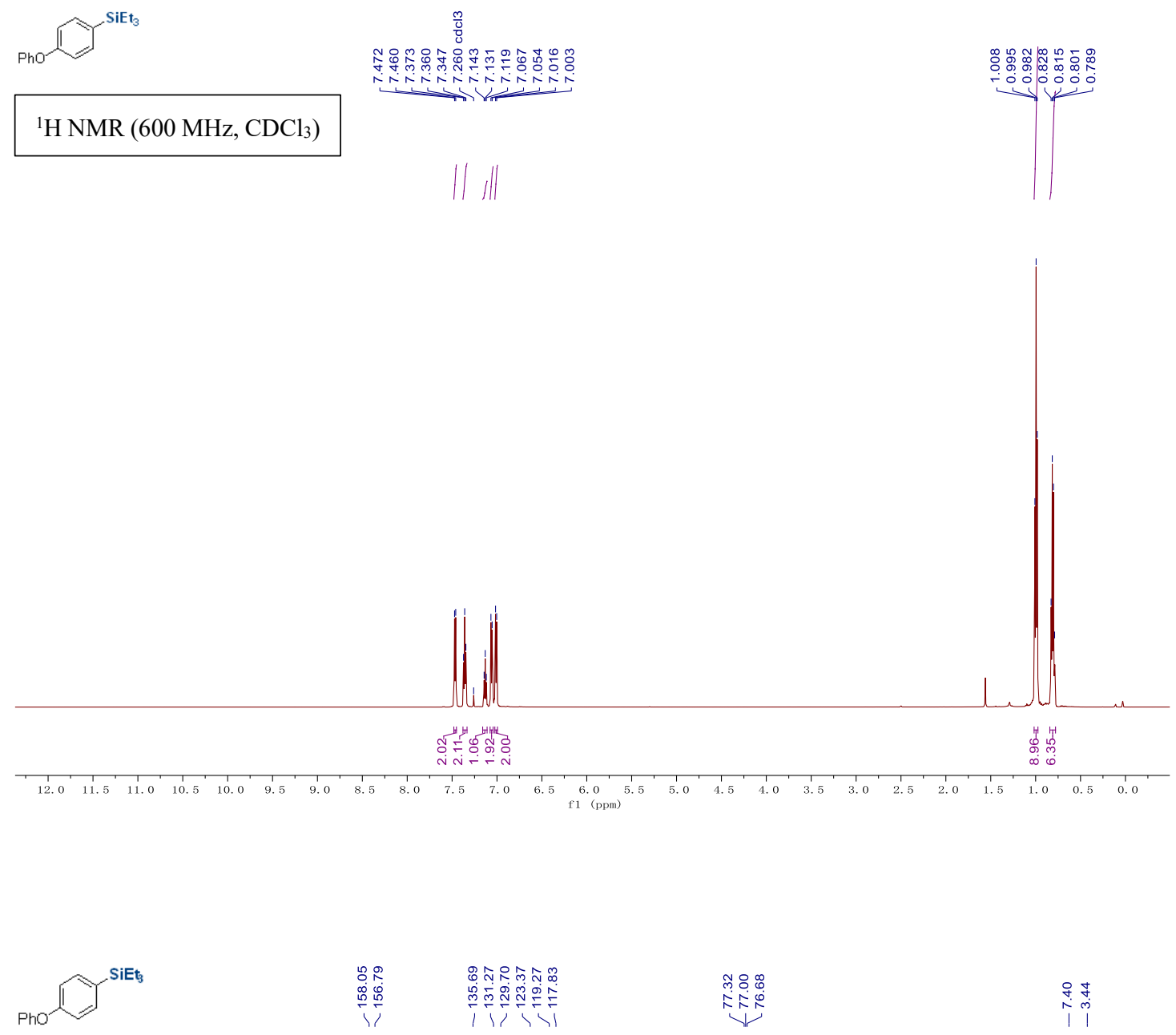

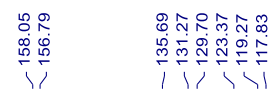

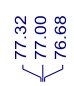

${ }^{13} \mathrm{C} \mathrm{NMR}\left(100 \mathrm{MHz}, \mathrm{CDCl}_{3}\right)$

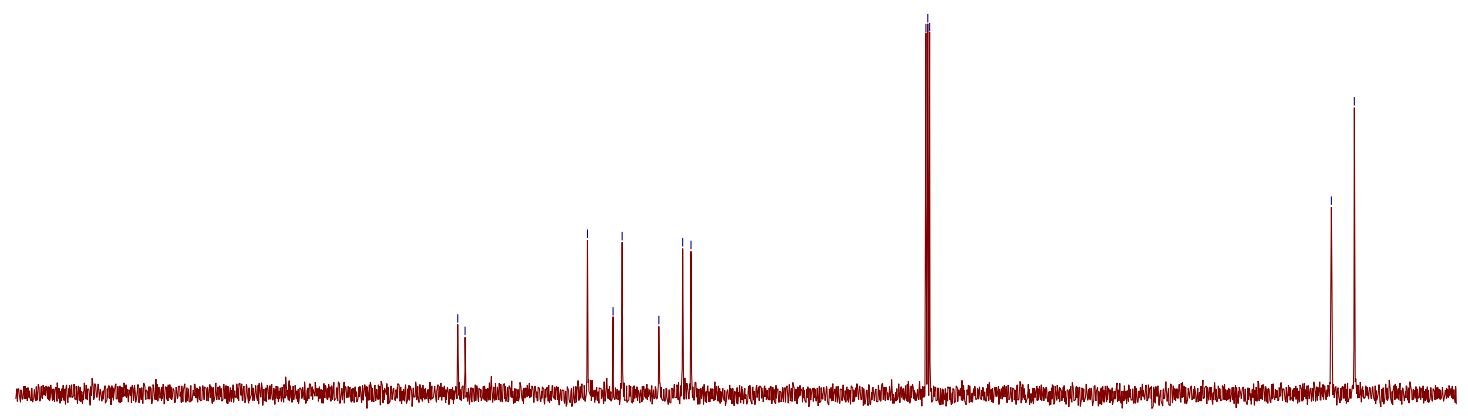

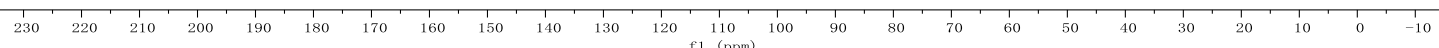




\section{(3,4-Dihydronaphthalen-1-yl)triethylsilane (32)}

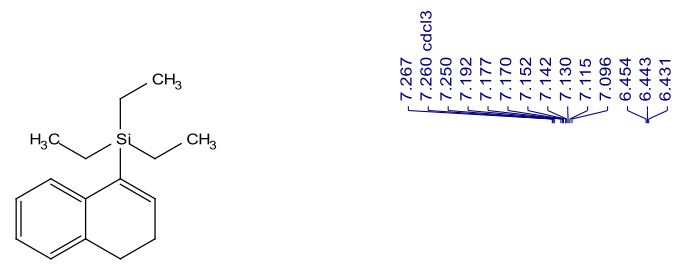

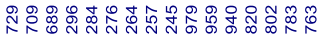

Nhin

$\mathrm{H}$ NMR $\left(400 \mathrm{MHz}, \mathrm{CDCl}_{3}\right)$

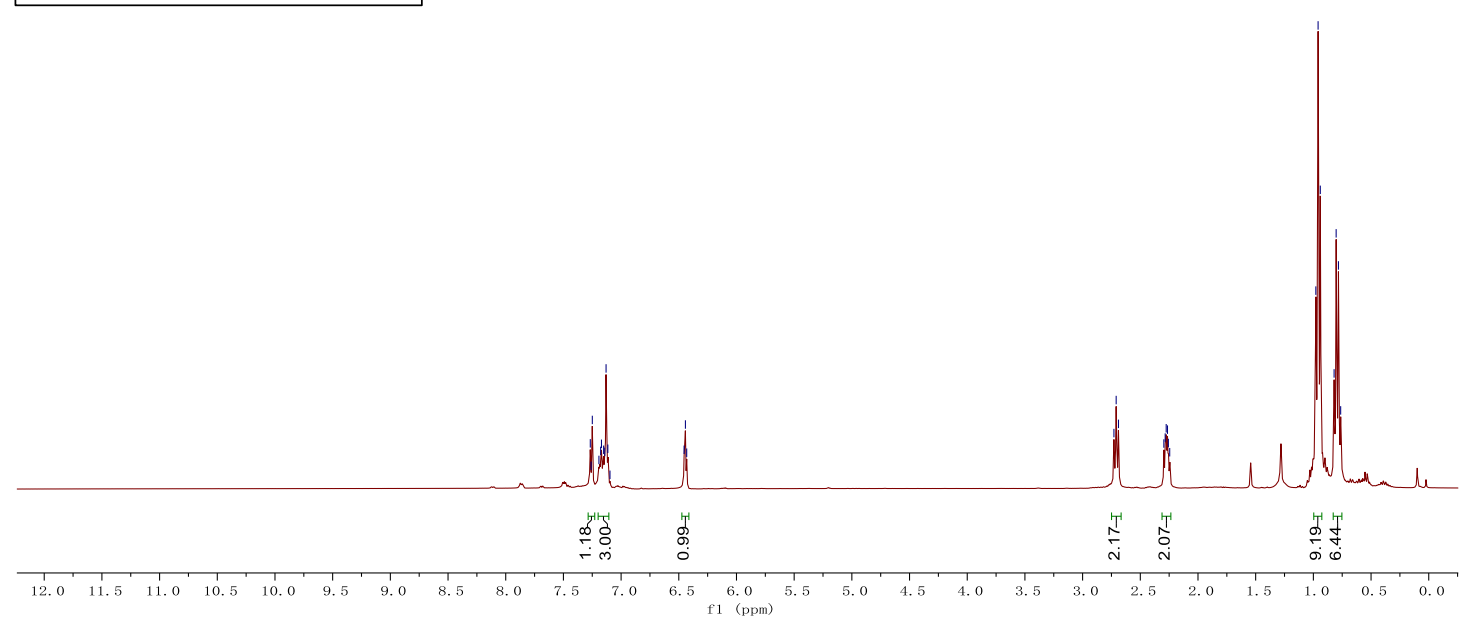

${ }^{13} \mathrm{C} \mathrm{NMR}\left(100 \mathrm{MHz}, \mathrm{CDCl}_{3}\right)$

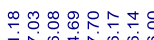

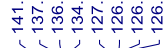

篎送

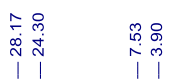

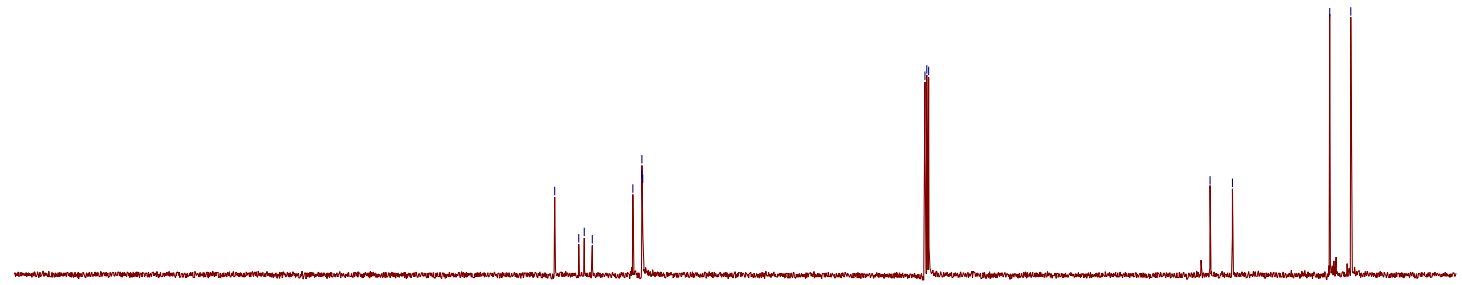

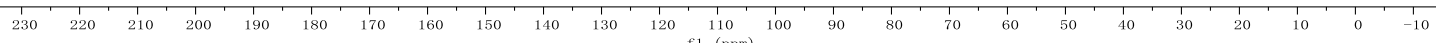


Triethyl(4-methyl-3,4-dihydronaphthalen-1-yl)silane (33)

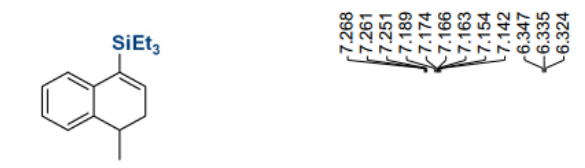

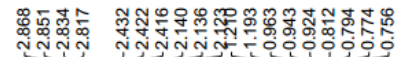

${ }^{1} \mathrm{H} \mathrm{NMR}\left(400 \mathrm{MHz}, \mathrm{CDCl}_{3}\right)$
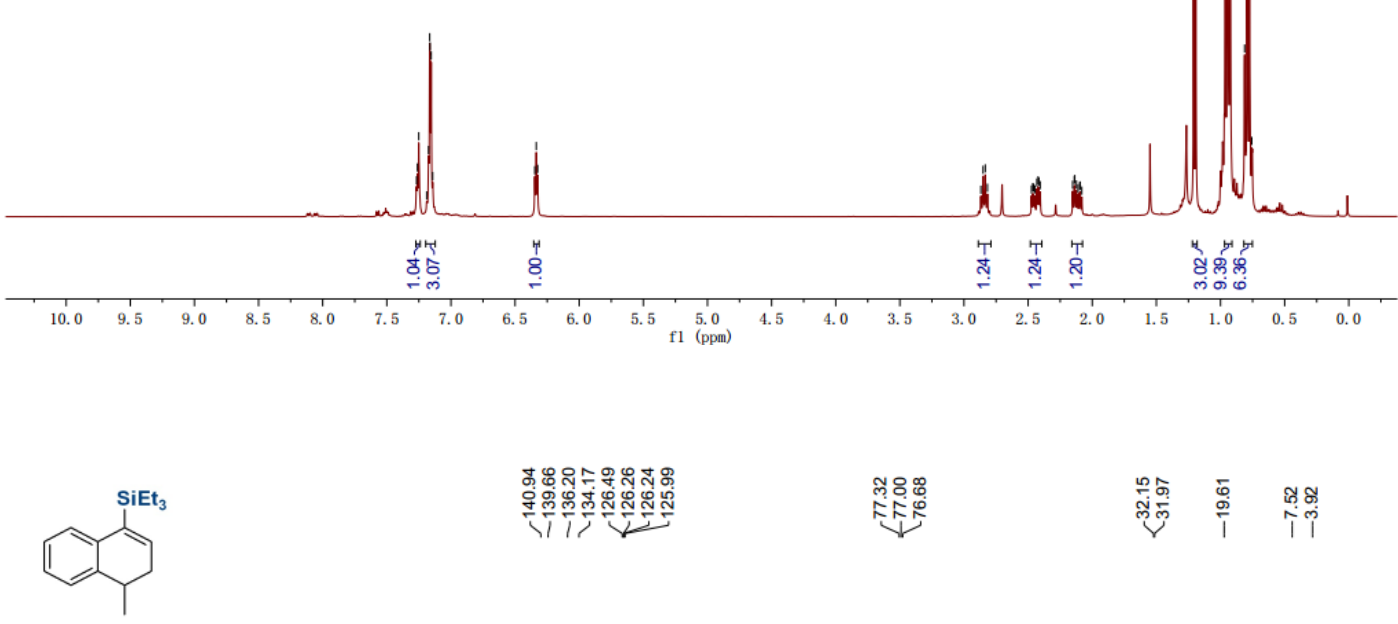

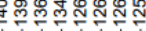

윰ำ

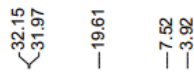

${ }^{13} \mathrm{C} \mathrm{NMR}\left(100 \mathrm{MHz}, \mathrm{CDCl}_{3}\right)$

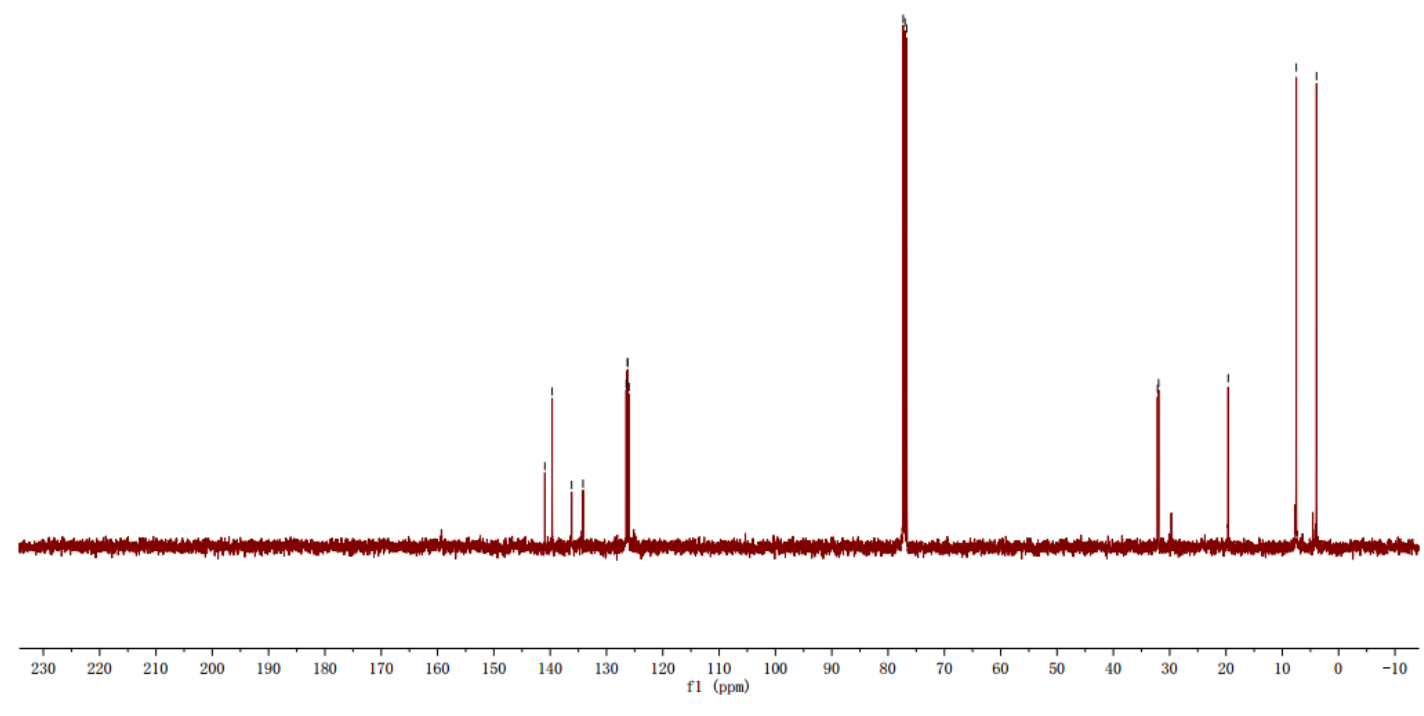


(5-(Benzyloxy)-3,4-dihydronaphthalen-1-yl)triethylsilane (34)

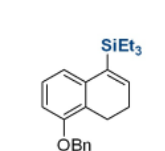

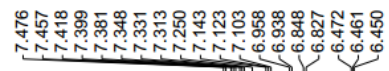

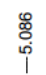

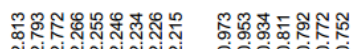

$\mathrm{OBn}$

${ }^{1} \mathrm{H} \mathrm{NMR}\left(400 \mathrm{MHz}, \mathrm{CDCl}_{3}\right)$
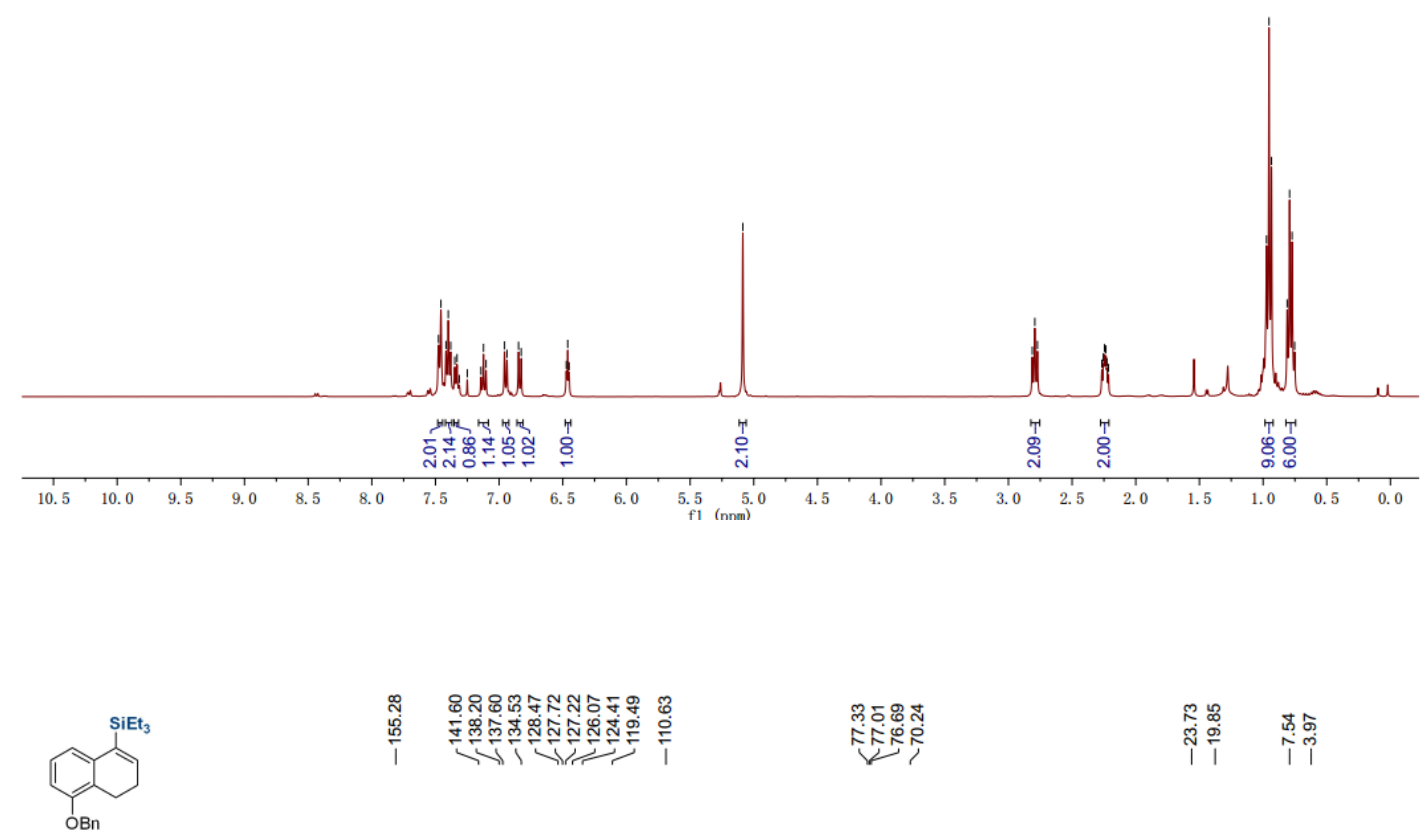

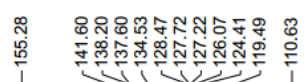

תू.

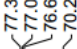

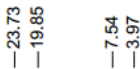

OBn

${ }^{13} \mathrm{C} \mathrm{NMR}\left(100 \mathrm{MHz}, \mathrm{CDCl}_{3}\right)$

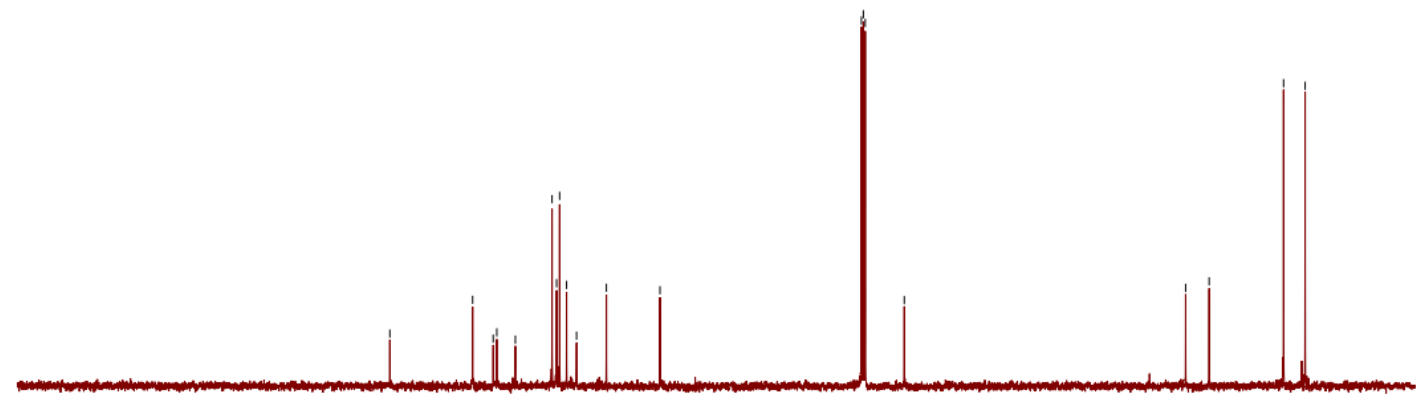

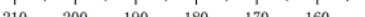
$\begin{array}{llllll}130 & 120 & 110 & 100 & 90\end{array}$ \begin{tabular}{rrrrrrrrrrr}
1 & 1 & 1 & 1 & 1 & 1 & 1 & 1 & 1 & 1 \\
\hline 0 & 70 & 60 & 50 & 40 & 30 & 20 & 10 & 0 & -10
\end{tabular} 
Triethyl(7-methyl-3,4-dihydronaphthalen-1-yl)silane (35)
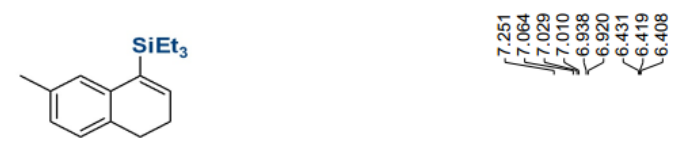

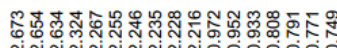

${ }^{1} \mathrm{H} \mathrm{NMR}\left(400 \mathrm{MHz}, \mathrm{CDCl}_{3}\right)$
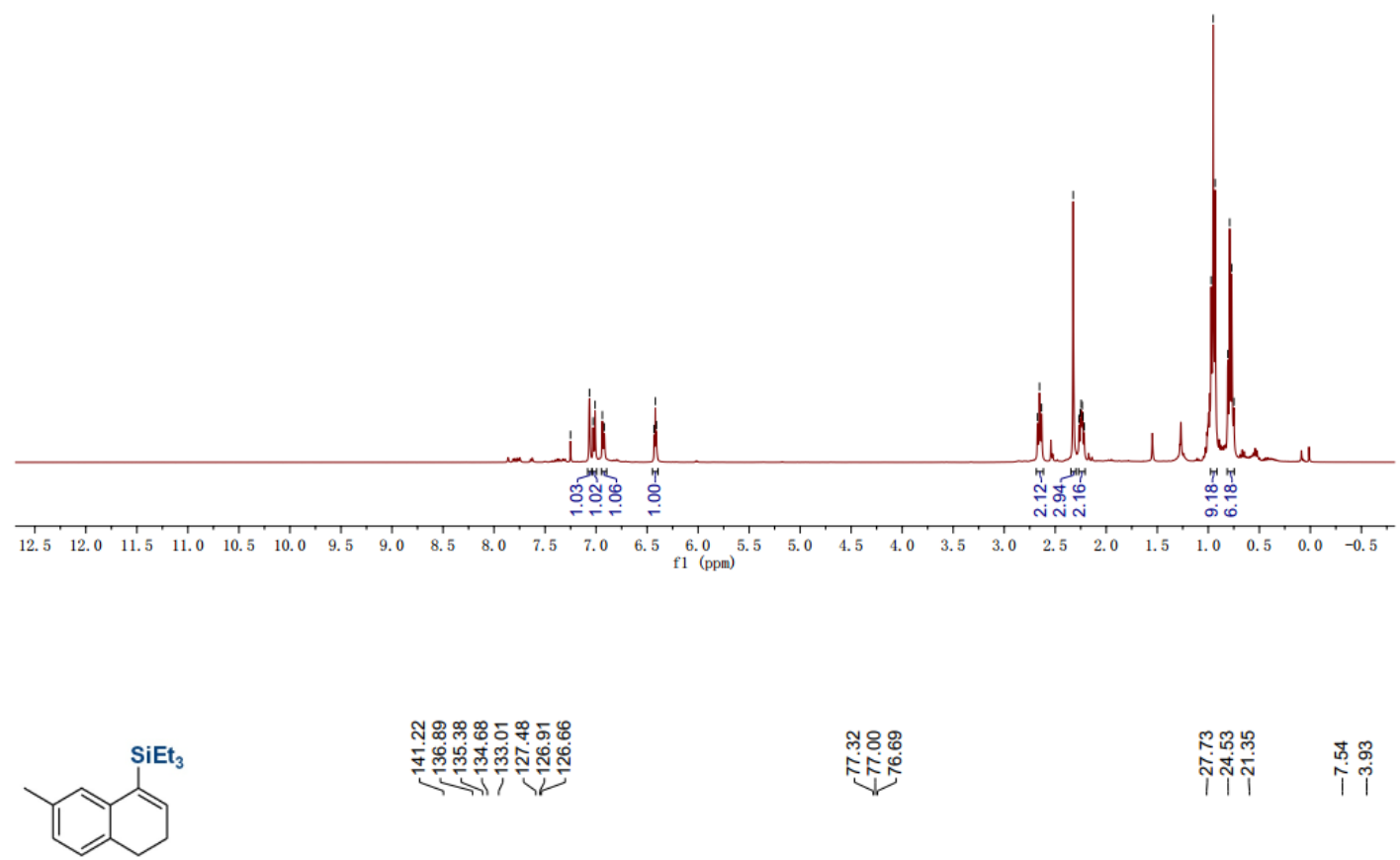

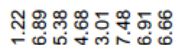

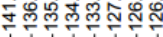

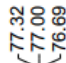

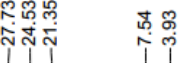

${ }^{13} \mathrm{C}$ NMR (100 MHz, $\left.\mathrm{CDCl}_{3}\right)$

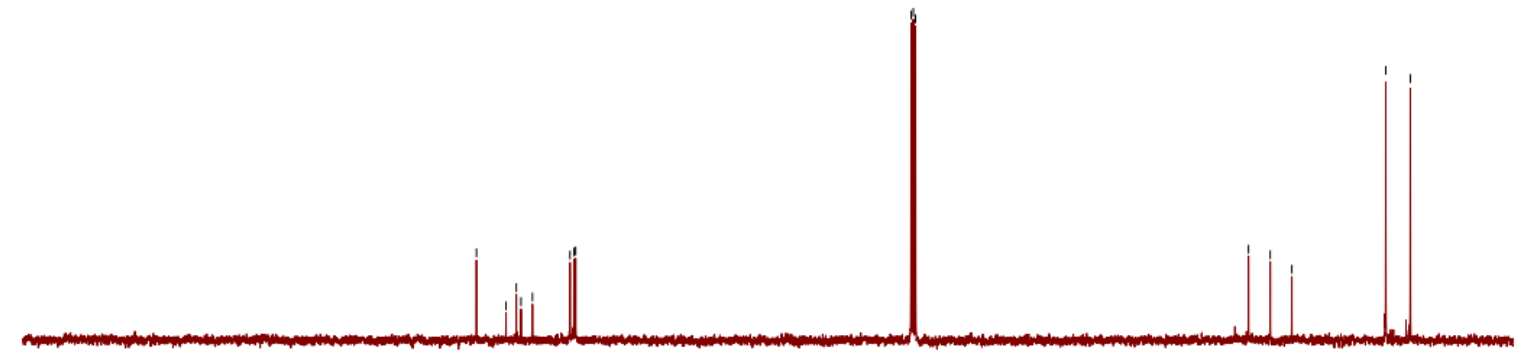

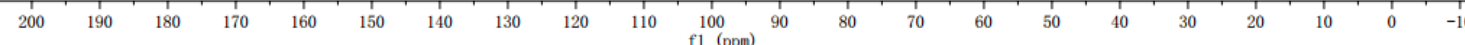


2-((8-(Triethylsilyl)-5,6-dihydronaphthalen-2-yl)oxy)pyridine (36)
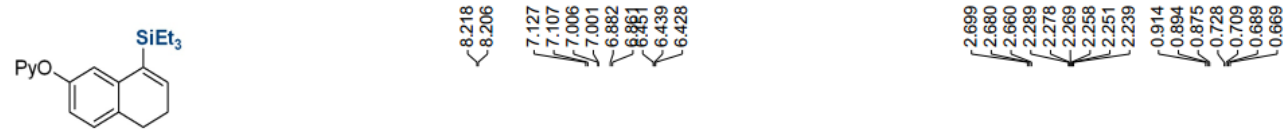

${ }^{1} \mathrm{H} \mathrm{NMR}\left(400 \mathrm{MHz}, \mathrm{CDCl}_{3}\right)$
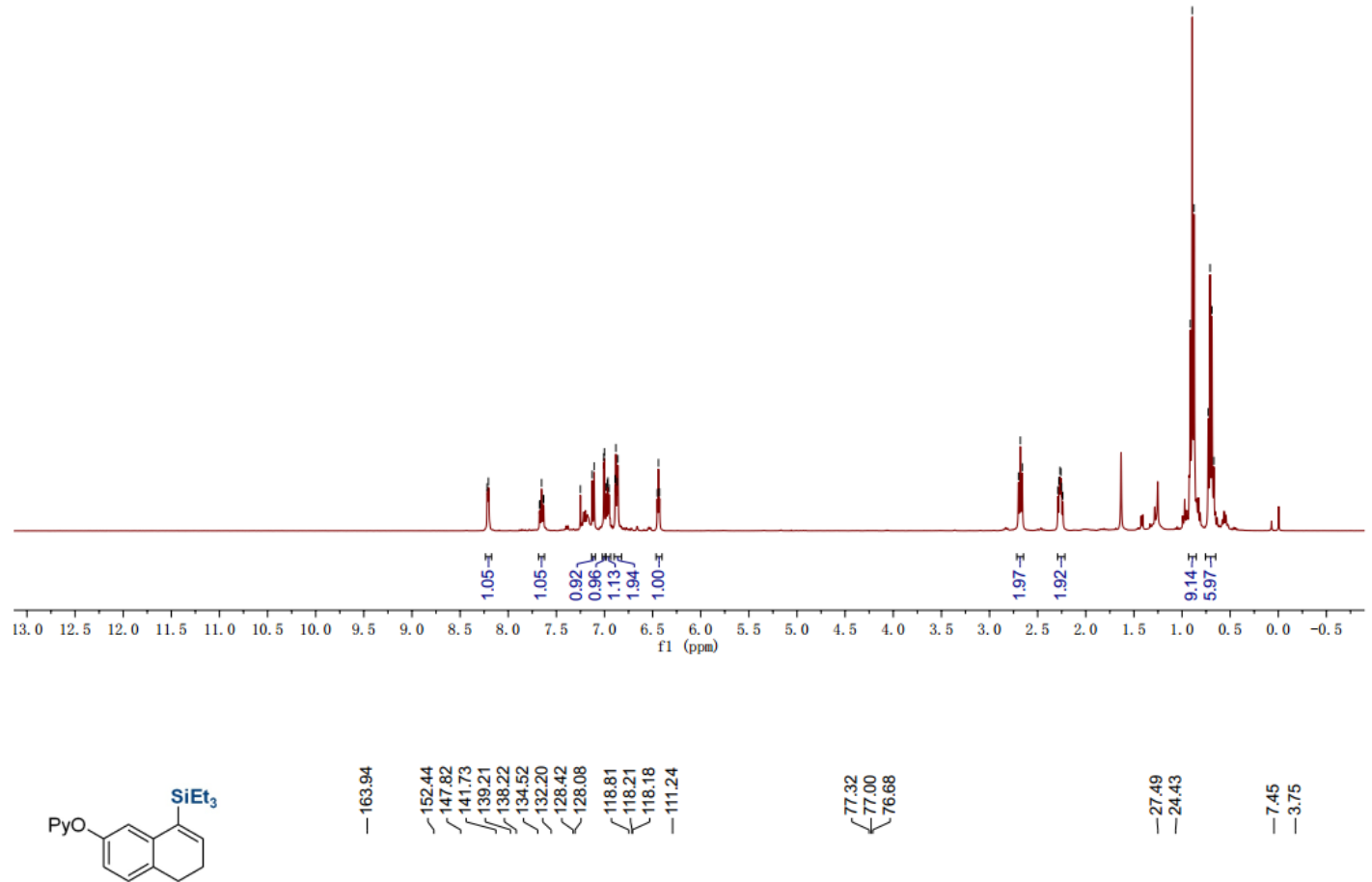

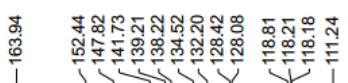

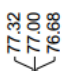

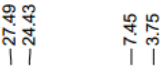

${ }^{13} \mathrm{C} \mathrm{NMR}\left(100 \mathrm{MHz}, \mathrm{CDCl}_{3}\right)$

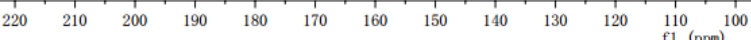


Triethyl(6-methoxy-3,4-dihydronaphthalen-1-yl)silane (37)

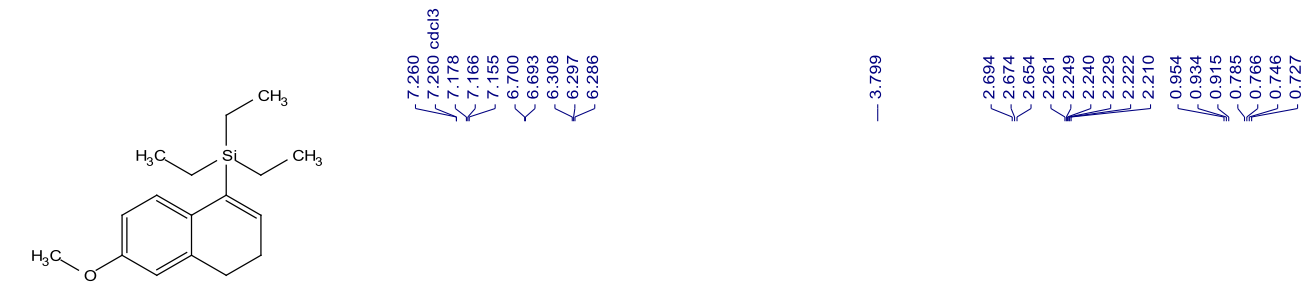

${ }^{1} \mathrm{H} \mathrm{NMR}\left(400 \mathrm{MHz}, \mathrm{CDCl}_{3}\right)$
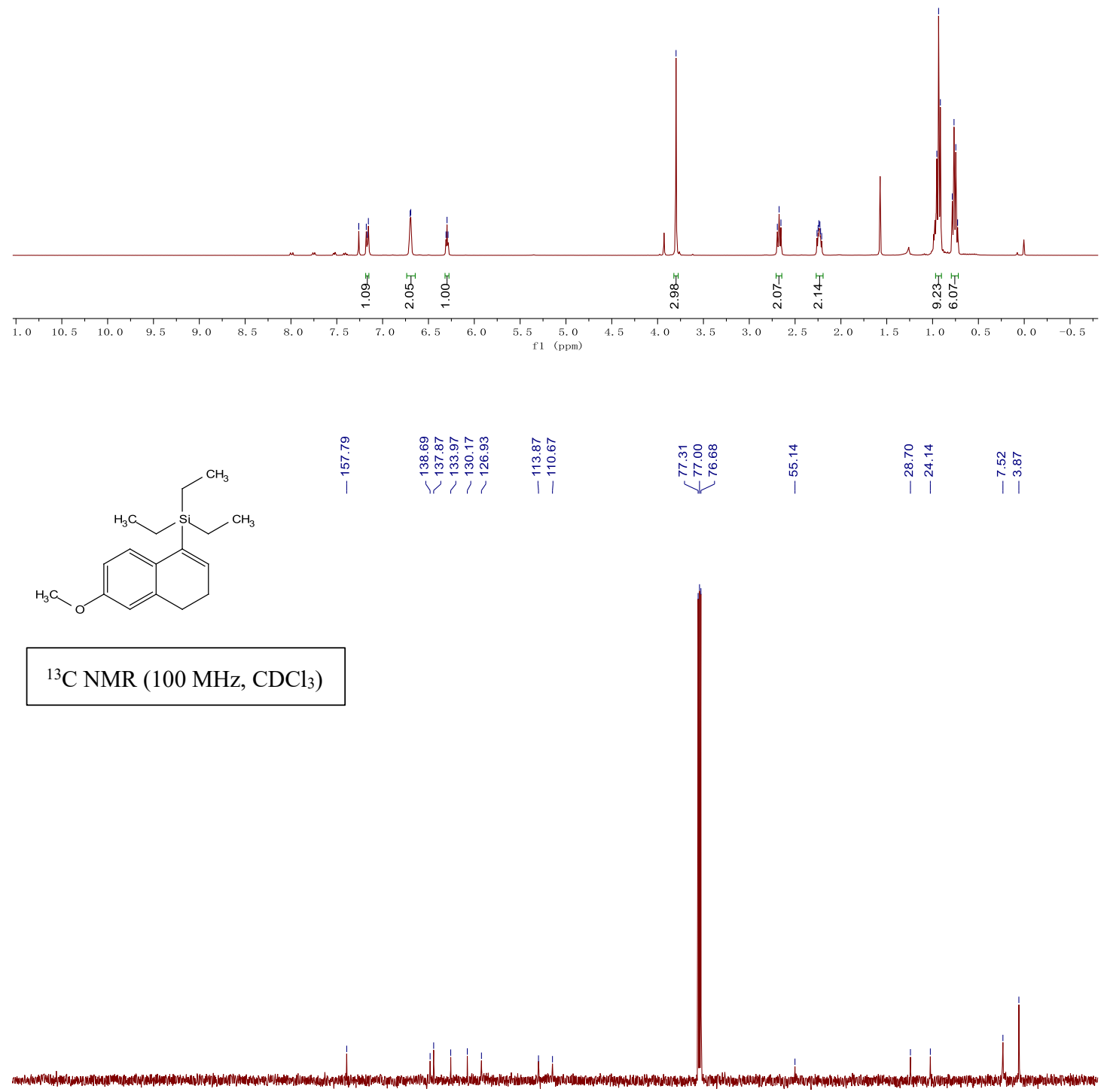

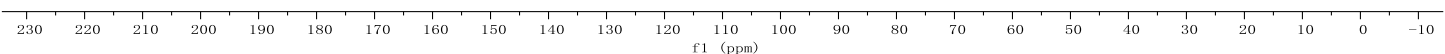


Triethyl(7-methoxy-3,4-dihydronaphthalen-1-yl)silane (38)
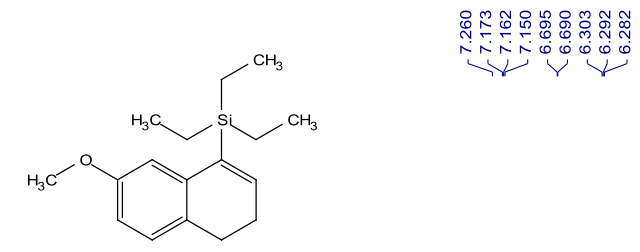

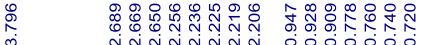

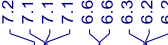

${ }^{1} \mathrm{H} \mathrm{NMR}\left(400 \mathrm{MHz}, \mathrm{CDCl}_{3}\right)$

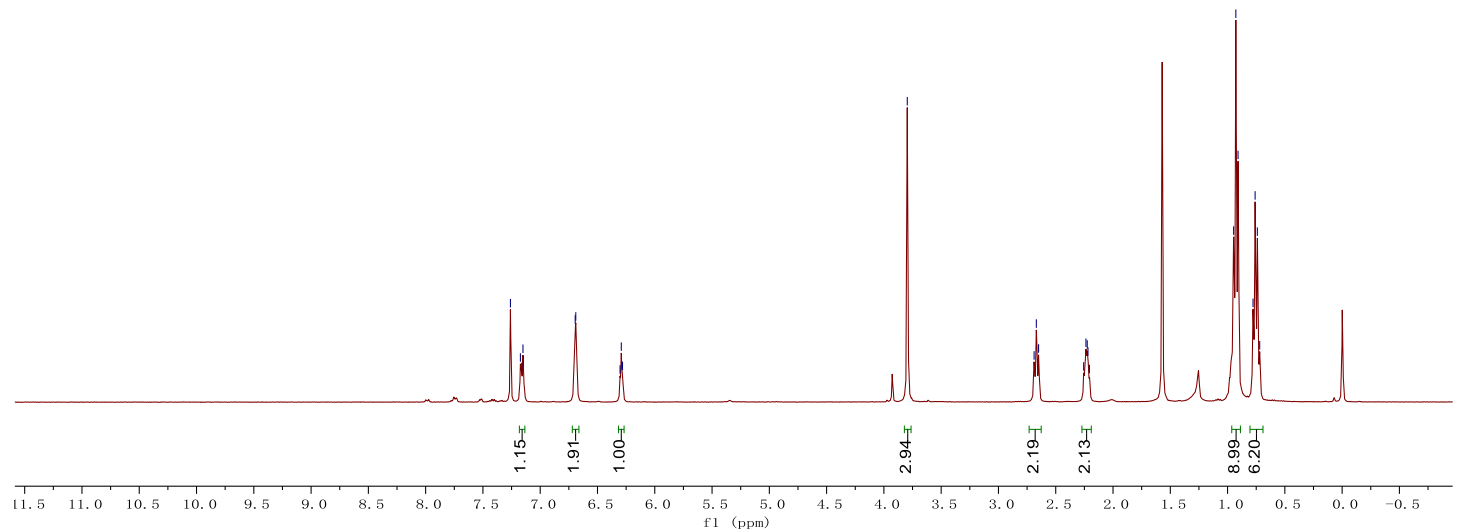

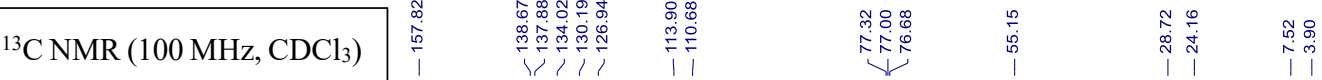

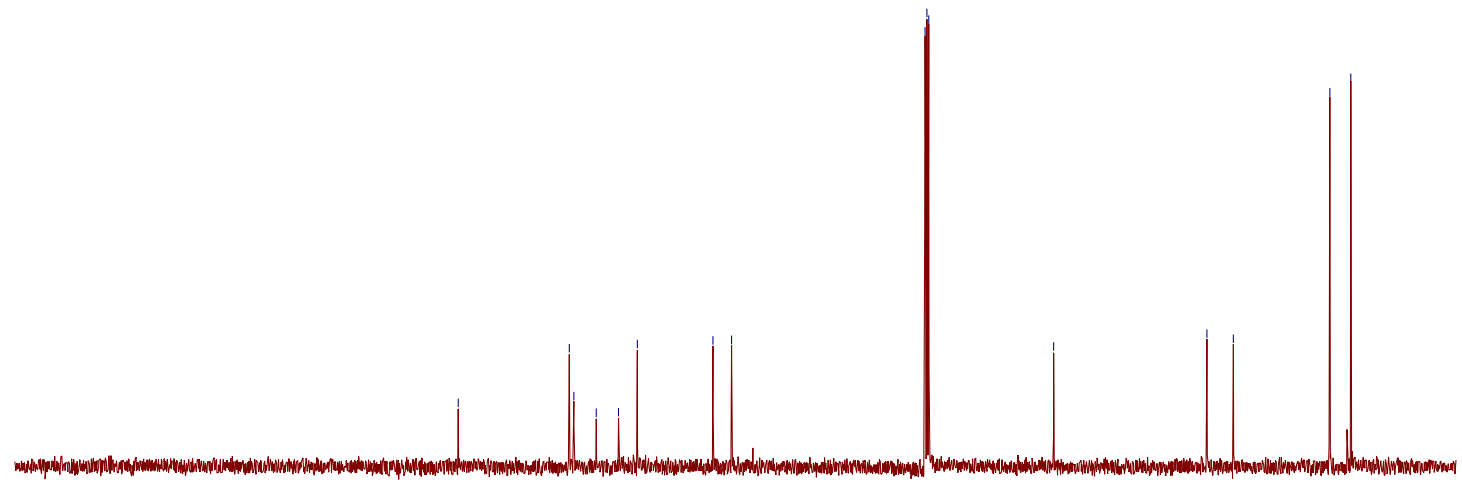

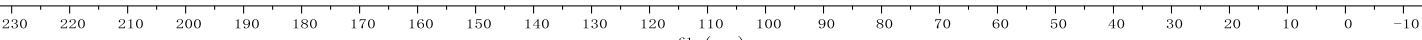


$\mathrm{N}, \mathrm{N}$-Dimethyl-5-(triethylsilyl)-7,8-dihydronaphthalen-2-amine (39)
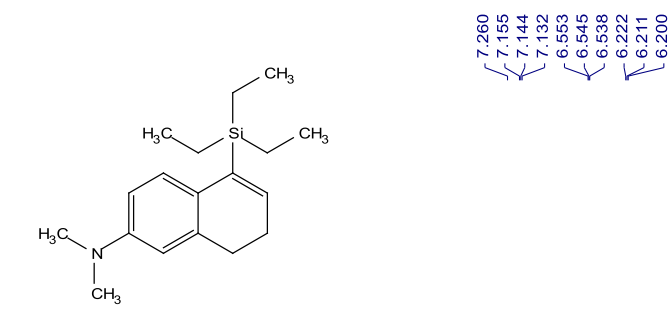

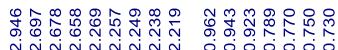

${ }^{1} \mathrm{H} \mathrm{NMR}\left(400 \mathrm{MHz}, \mathrm{CDCl}_{3}\right)$
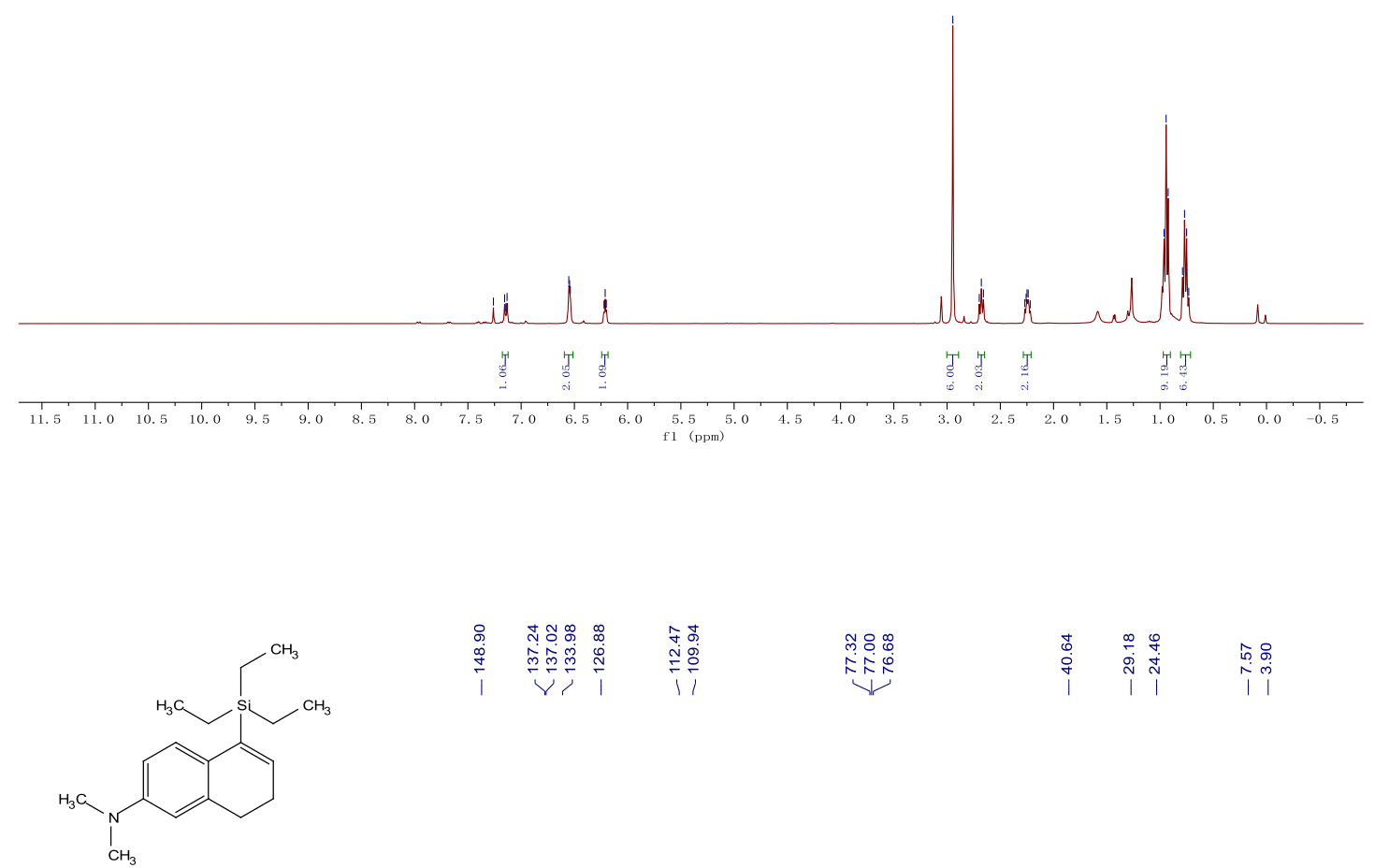

J

${ }^{13} \mathrm{C} \mathrm{NMR}\left(100 \mathrm{MHz}, \mathrm{CDCl}_{3}\right)$

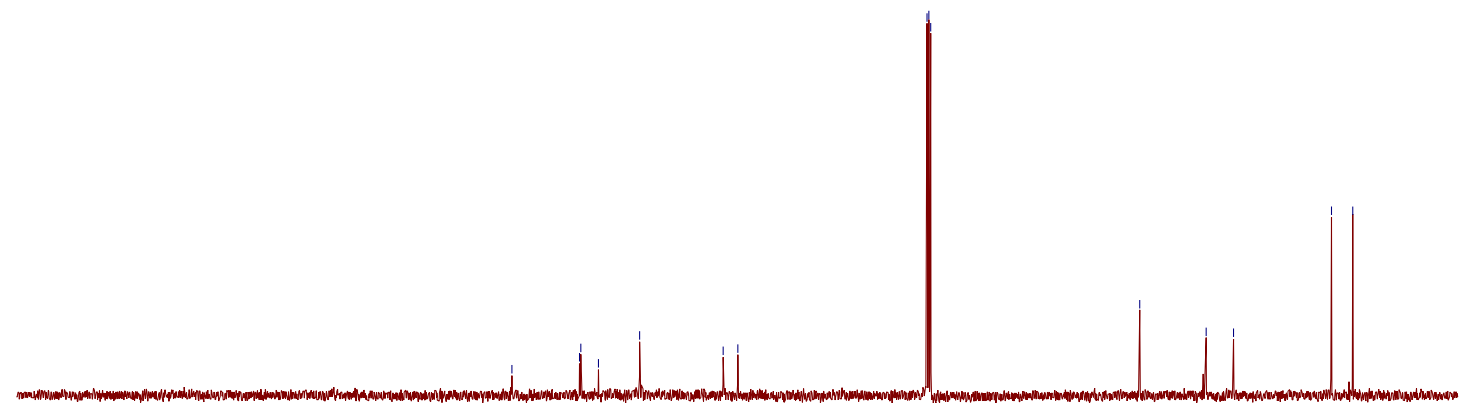

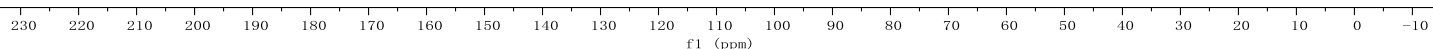


(1,3-diphenylprop-1-en-2-yl)triethylsilane (40)
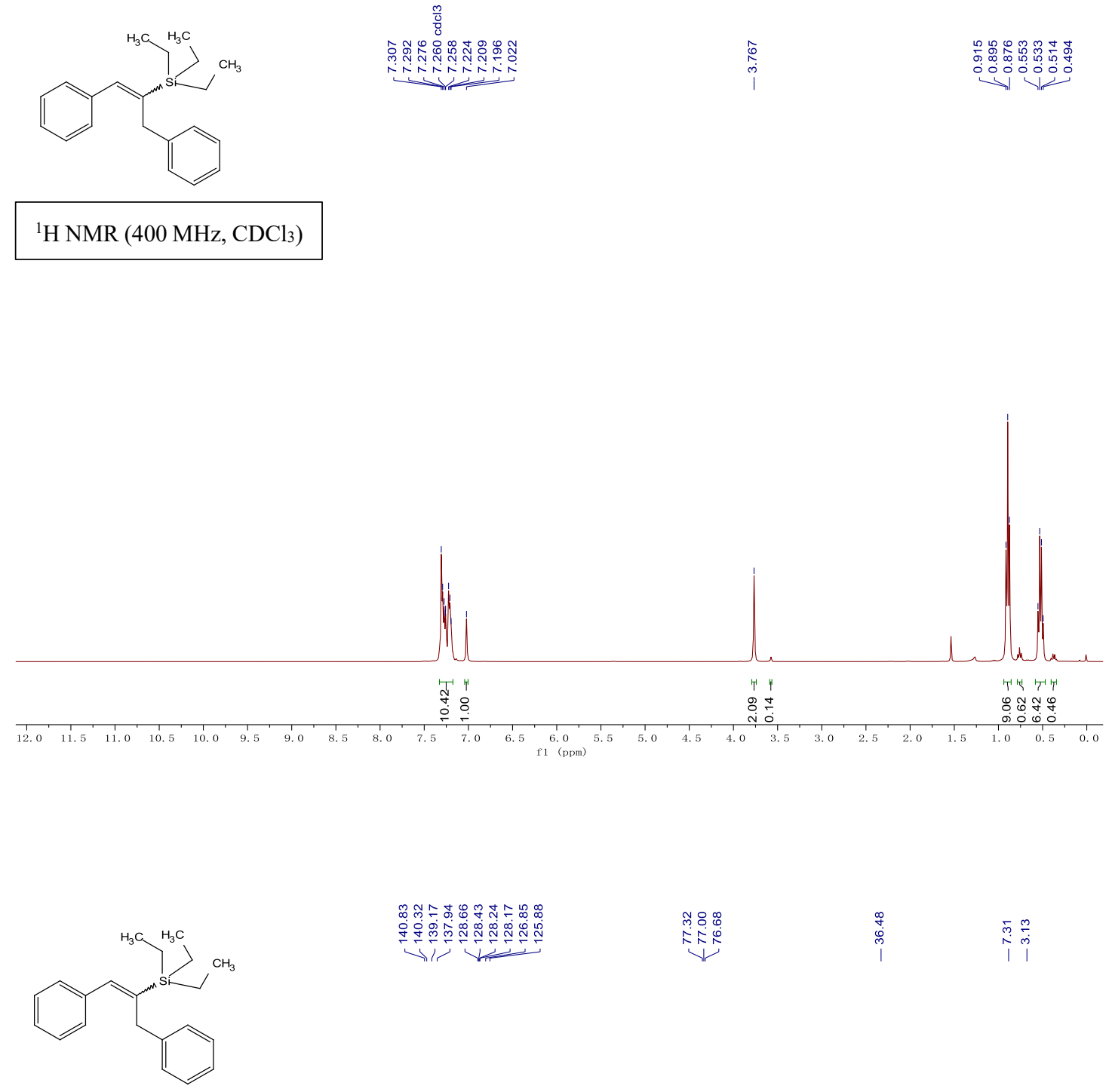

${ }^{13} \mathrm{C} \mathrm{NMR}\left(100 \mathrm{MHz}, \mathrm{CDCl}_{3}\right)$

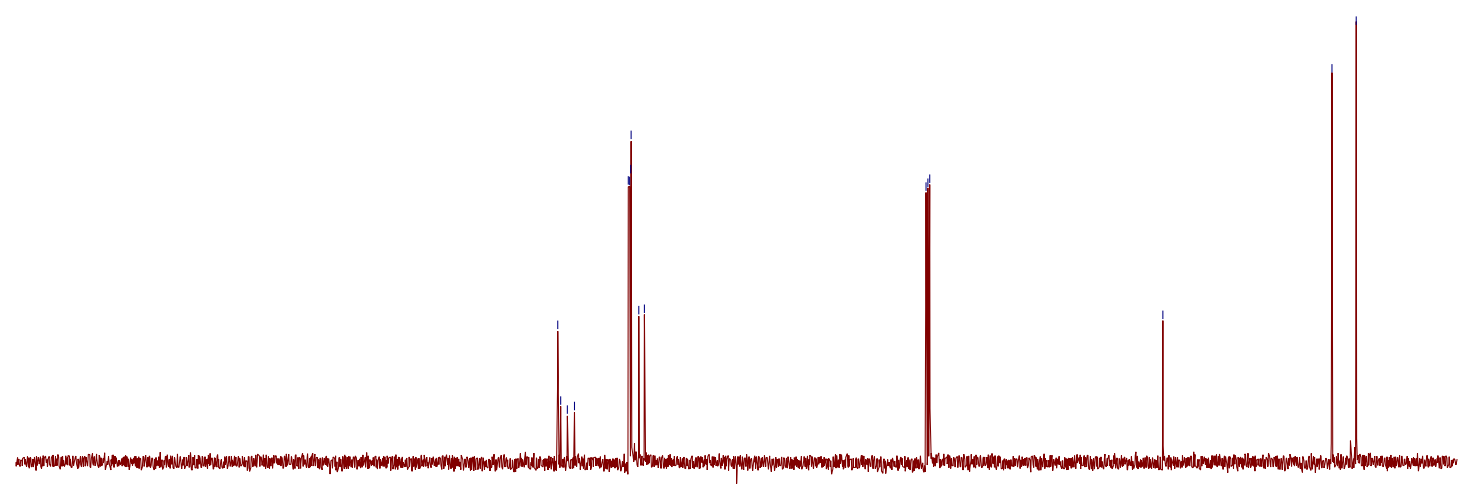


(3,4-Dihydronaphthalen-2-yl)triethylsilane (41)

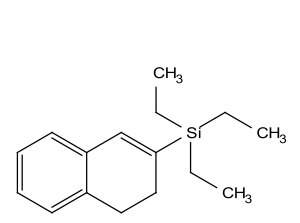

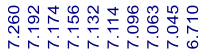

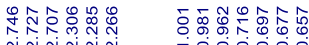

rojoio

${ }^{1} \mathrm{H}$ NMR $\left(400 \mathrm{MHz}, \mathrm{CDCl}_{3}\right)$

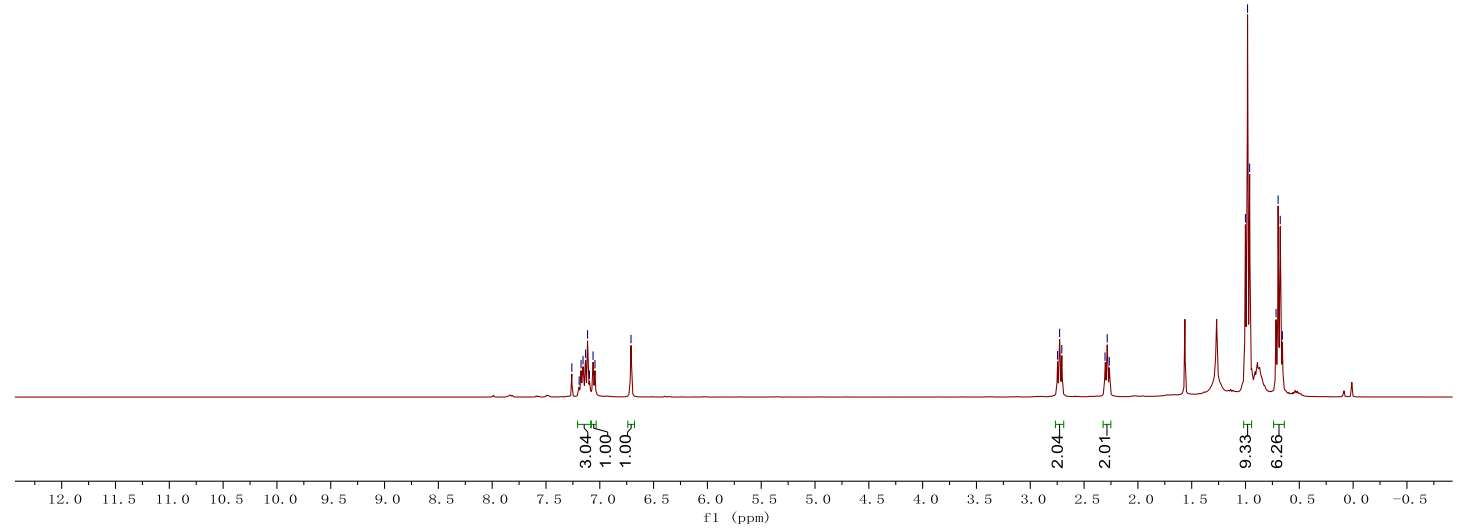

${ }^{13} \mathrm{C} \mathrm{NMR}\left(100 \mathrm{MHz}, \mathrm{CDCl}_{3}\right) \quad \begin{aligned} & \mathrm{m} \\ & 0\end{aligned}$

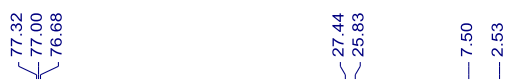

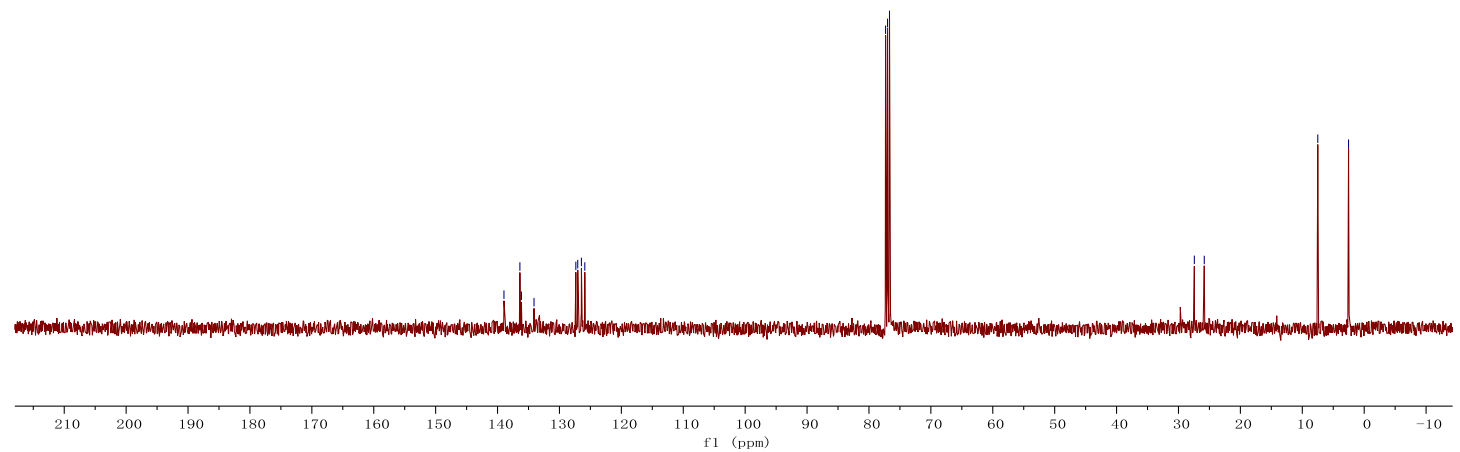


Triethyl(7-methoxy-3,4-dihydronaphthalen-2-yl)silane (42)

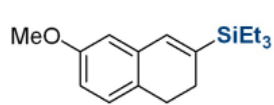

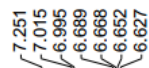

$\underset{\substack{\infty \\ \infty}}{\infty}$

递过通通

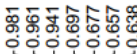

${ }^{1} \mathrm{H}$ NMR $\left(400 \mathrm{MHz}, \mathrm{CDCl}_{3}\right)$

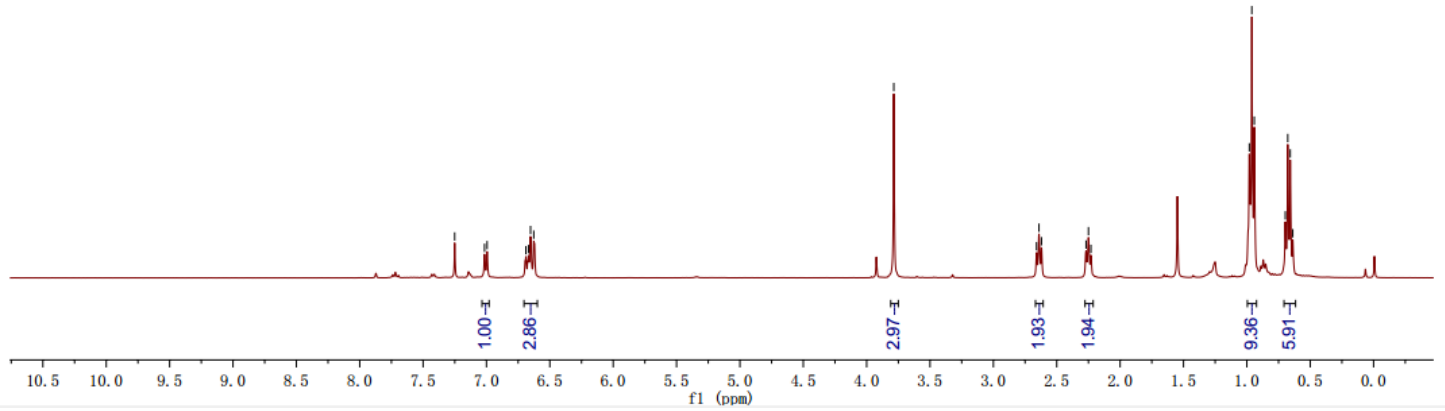

MeON SiEt $_{3}$ i

${ }^{13} \mathrm{C}$ NMR $\left(100 \mathrm{MHz}, \mathrm{CDCl}_{3}\right)$

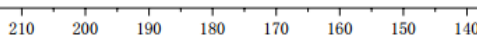

f1 $\stackrel{100}{(\mathrm{ppm})}$ 
(6,7-Dihydro-5H-benzo[7]annulen-9-yl)triethylsilane (43)

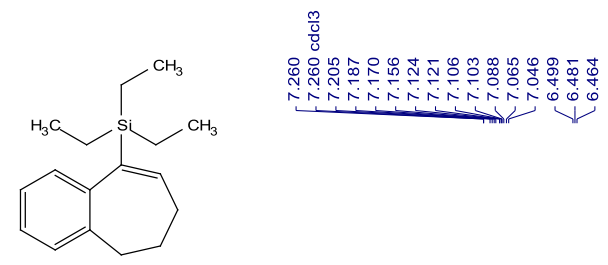

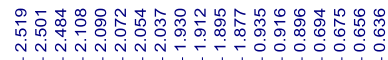

${ }^{1} \mathrm{H} \mathrm{NMR}\left(400 \mathrm{MHz}, \mathrm{CDCl}_{3}\right)$
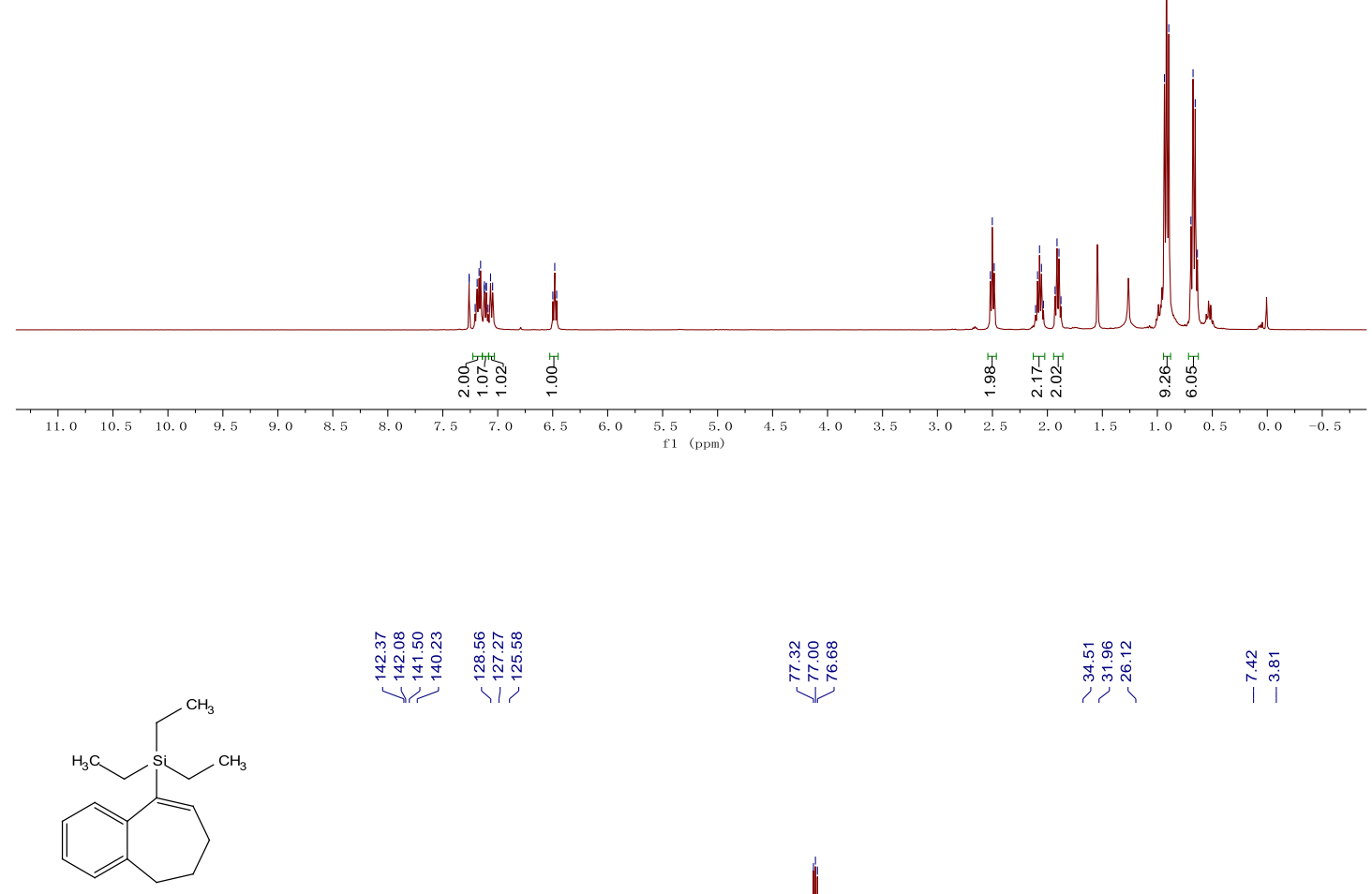

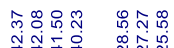

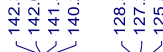

年

$\sqrt[5]{10}$

${ }^{13} \mathrm{C} \mathrm{NMR}\left(100 \mathrm{MHz}, \mathrm{CDCl}_{3}\right)$

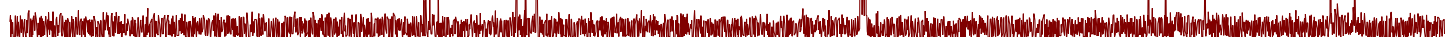

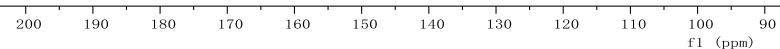


Triethyl(7-(3-methoxyphenyl)-3,4-dihydronaphthalen-1-yl)silane (44)

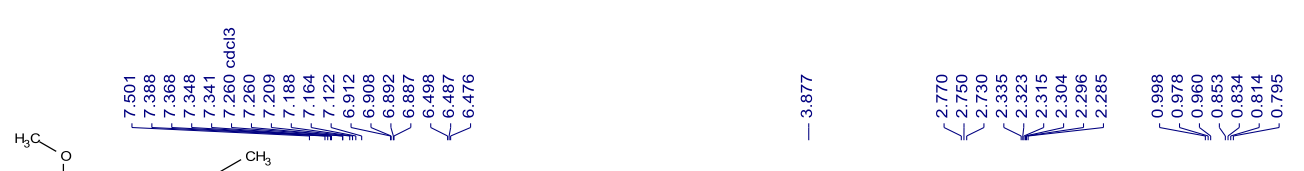

${ }^{1} \mathrm{H} \mathrm{NMR}\left(400 \mathrm{MHz}, \mathrm{CDCl}_{3}\right)$
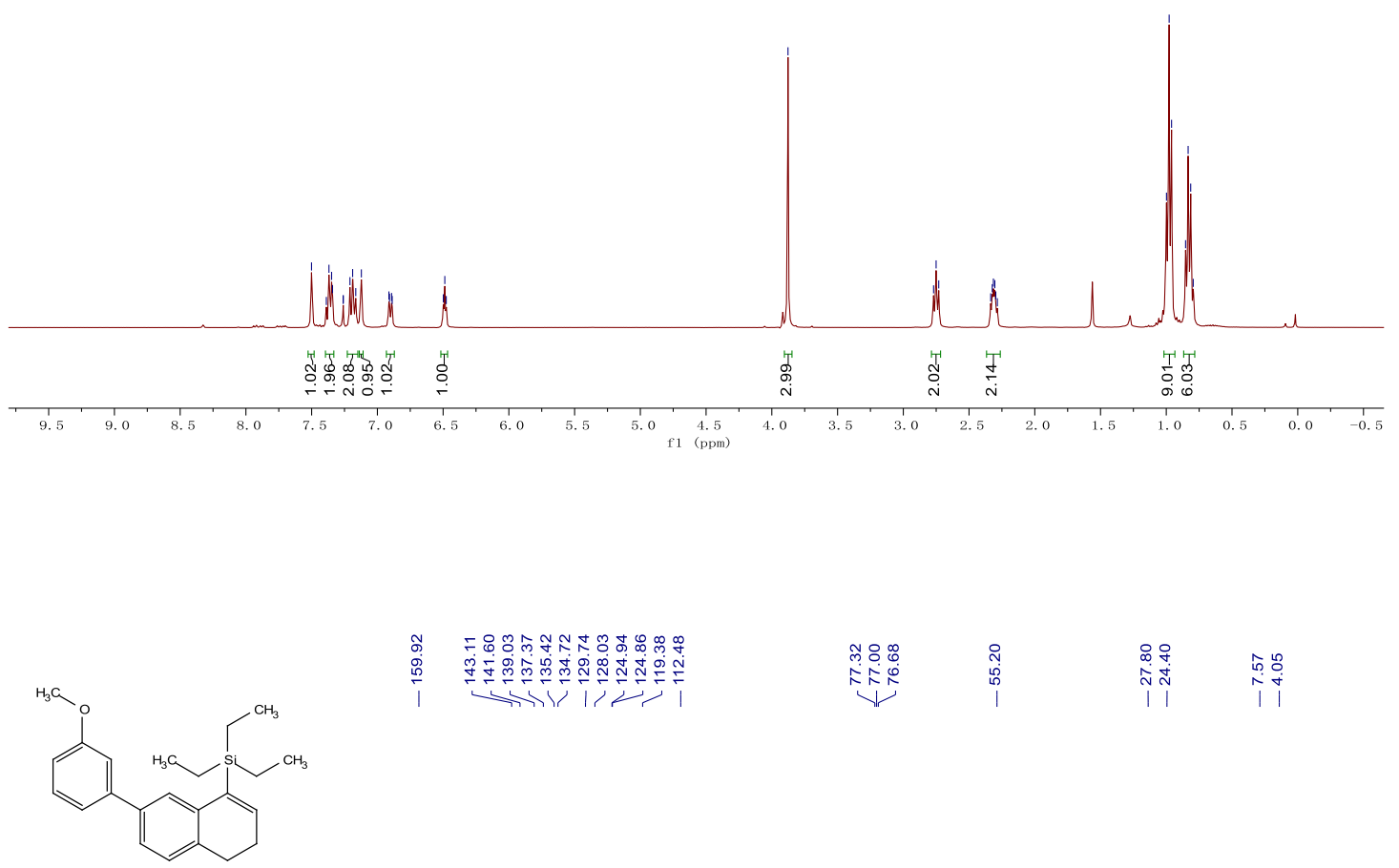

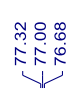

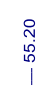

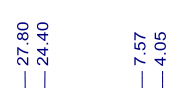

${ }^{13} \mathrm{C} \mathrm{NMR}\left(100 \mathrm{MHz}, \mathrm{CDCl}_{3}\right)$

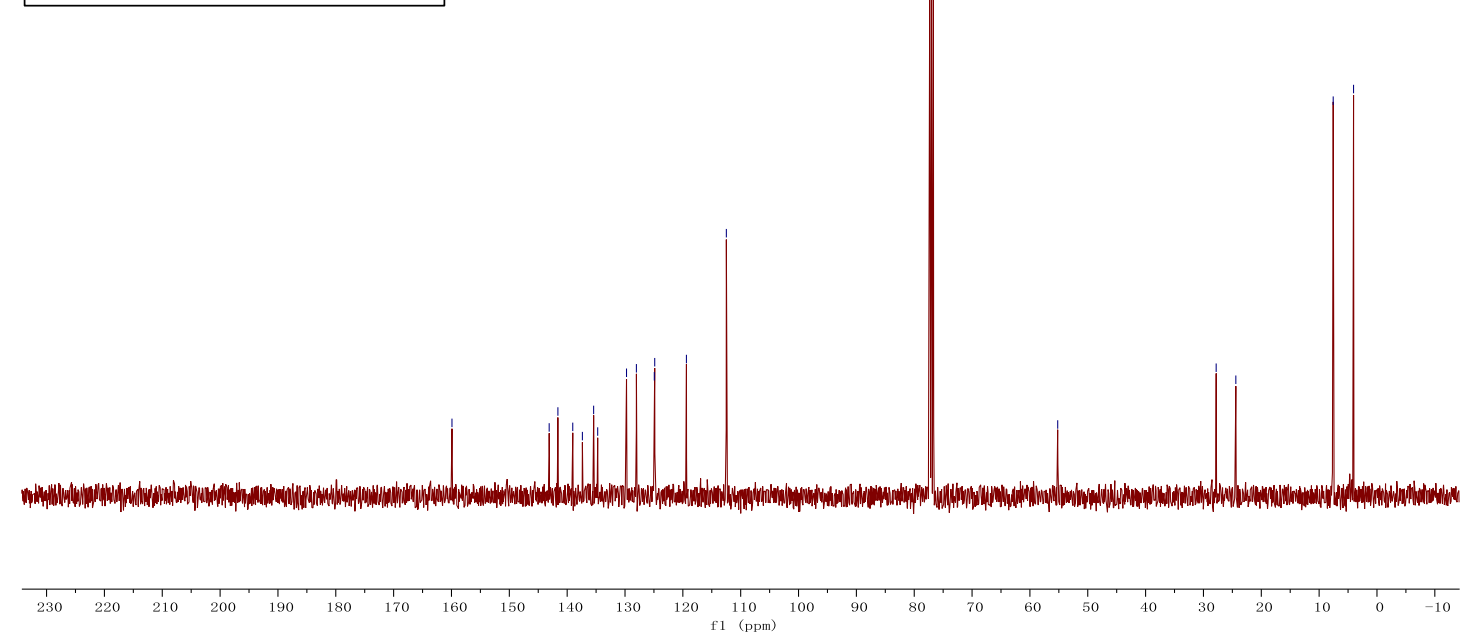

S79 
Triethyl(7-(4-(trifluoromethyl)phenyl)-3,4-dihydronaphthalen-1-yl)silane (45)
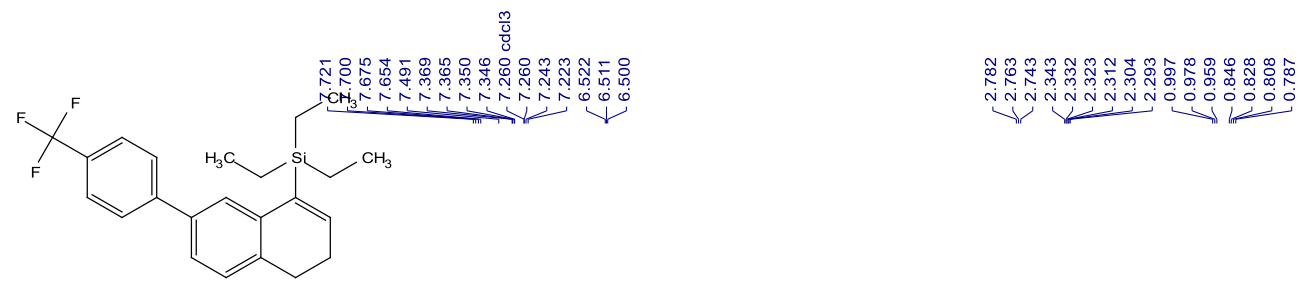

${ }^{1} \mathrm{H}$ NMR $\left(400 \mathrm{MHz}, \mathrm{CDCl}_{3}\right)$
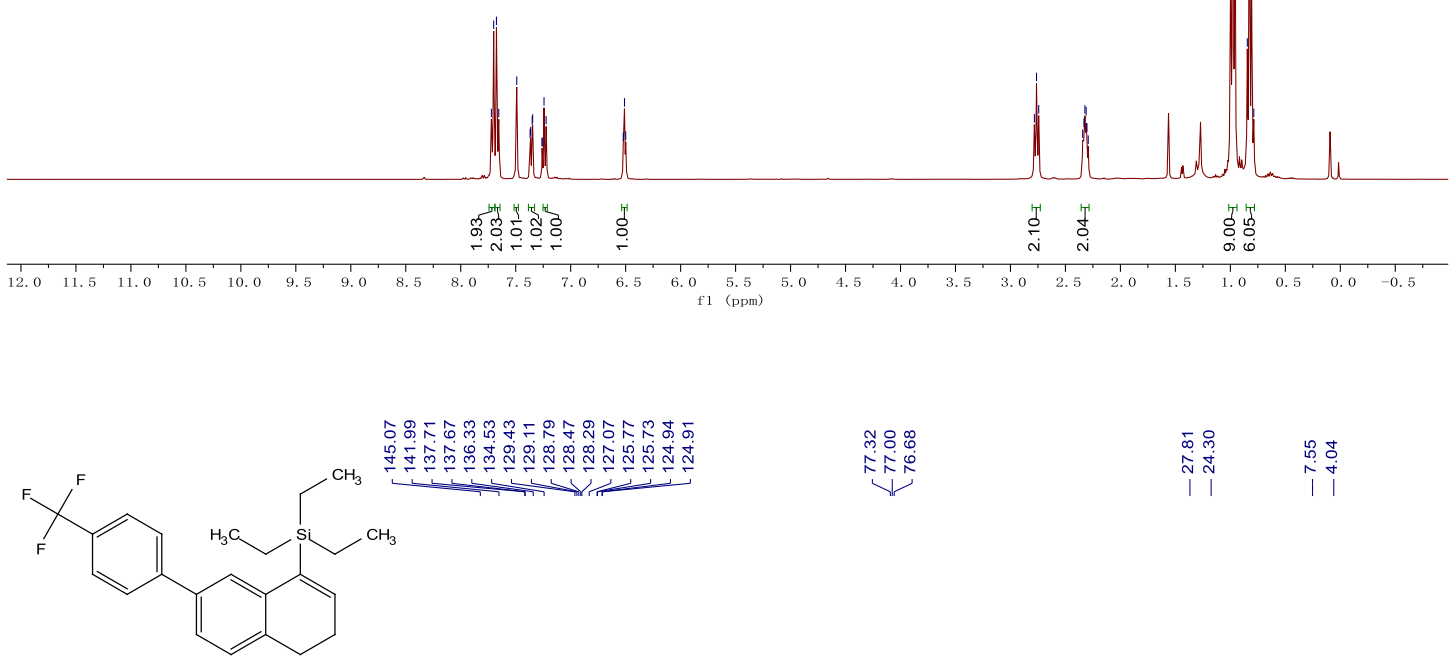

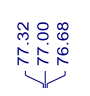

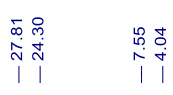

${ }^{13} \mathrm{C} \mathrm{NMR}\left(100 \mathrm{MHz}, \mathrm{CDCl}_{3}\right)$

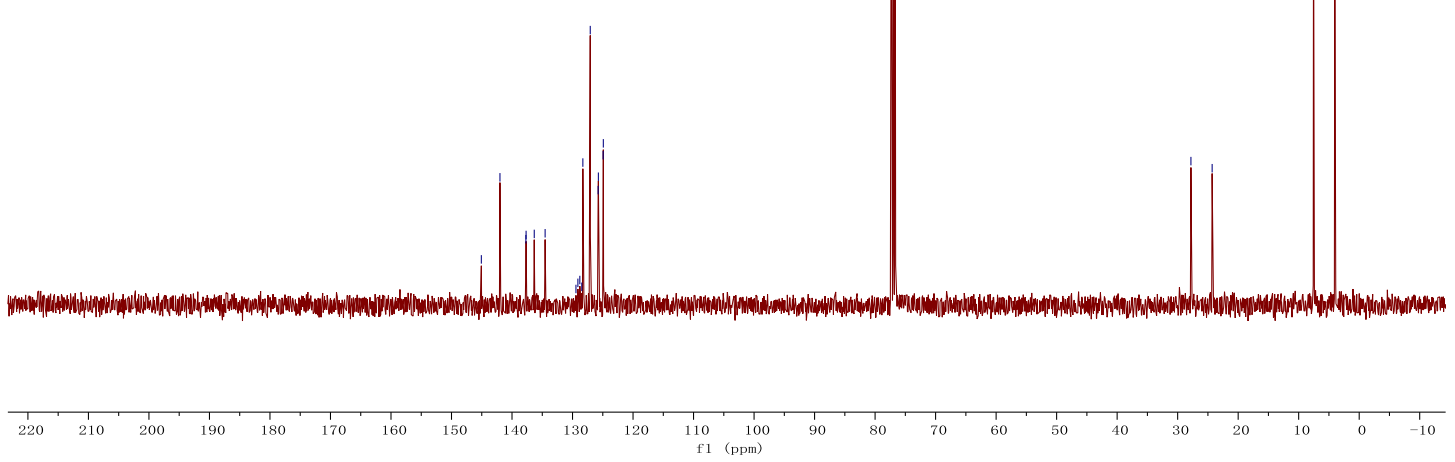

S80 
(7-Chloro-3,4-dihydronaphthalen-1-yl)triethylsilane (46)

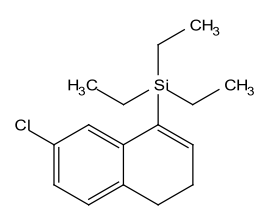

$\frac{\circ}{8}$

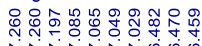

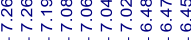

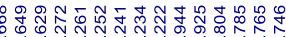

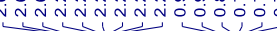

${ }^{1} \mathrm{H} \mathrm{NMR}\left(400 \mathrm{MHz}, \mathrm{CDCl}_{3}\right)$

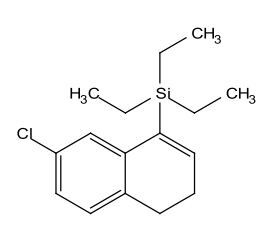

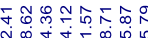

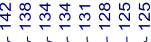

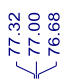

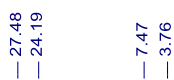

${ }^{13} \mathrm{C}$ NMR $\left(100 \mathrm{MHz}, \mathrm{CDCl}_{3}\right)$

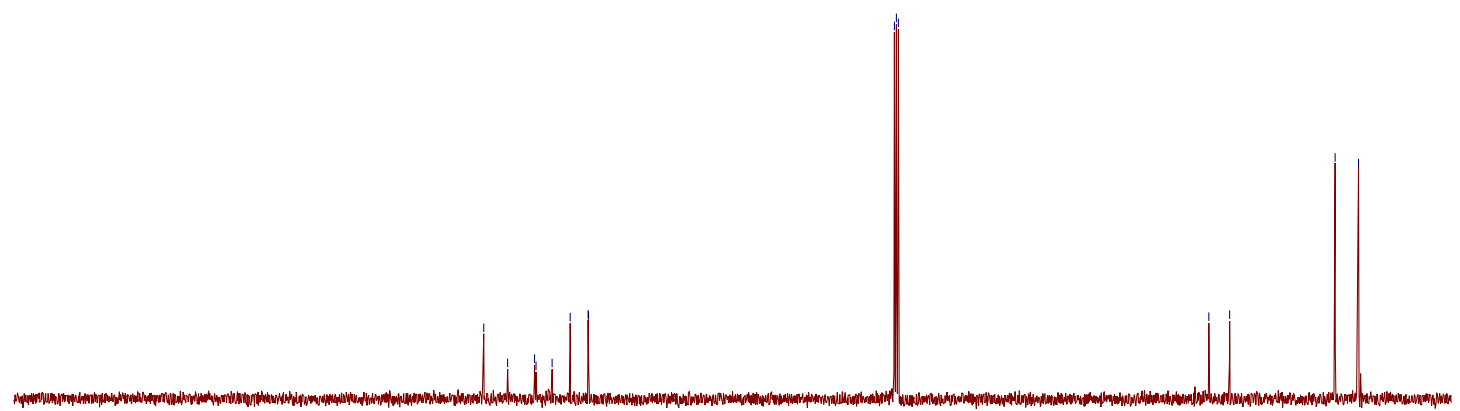

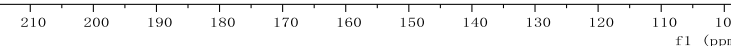


Triethyl(7-fluoro-3,4-dihydronaphthalen-1-yl)silane (47)

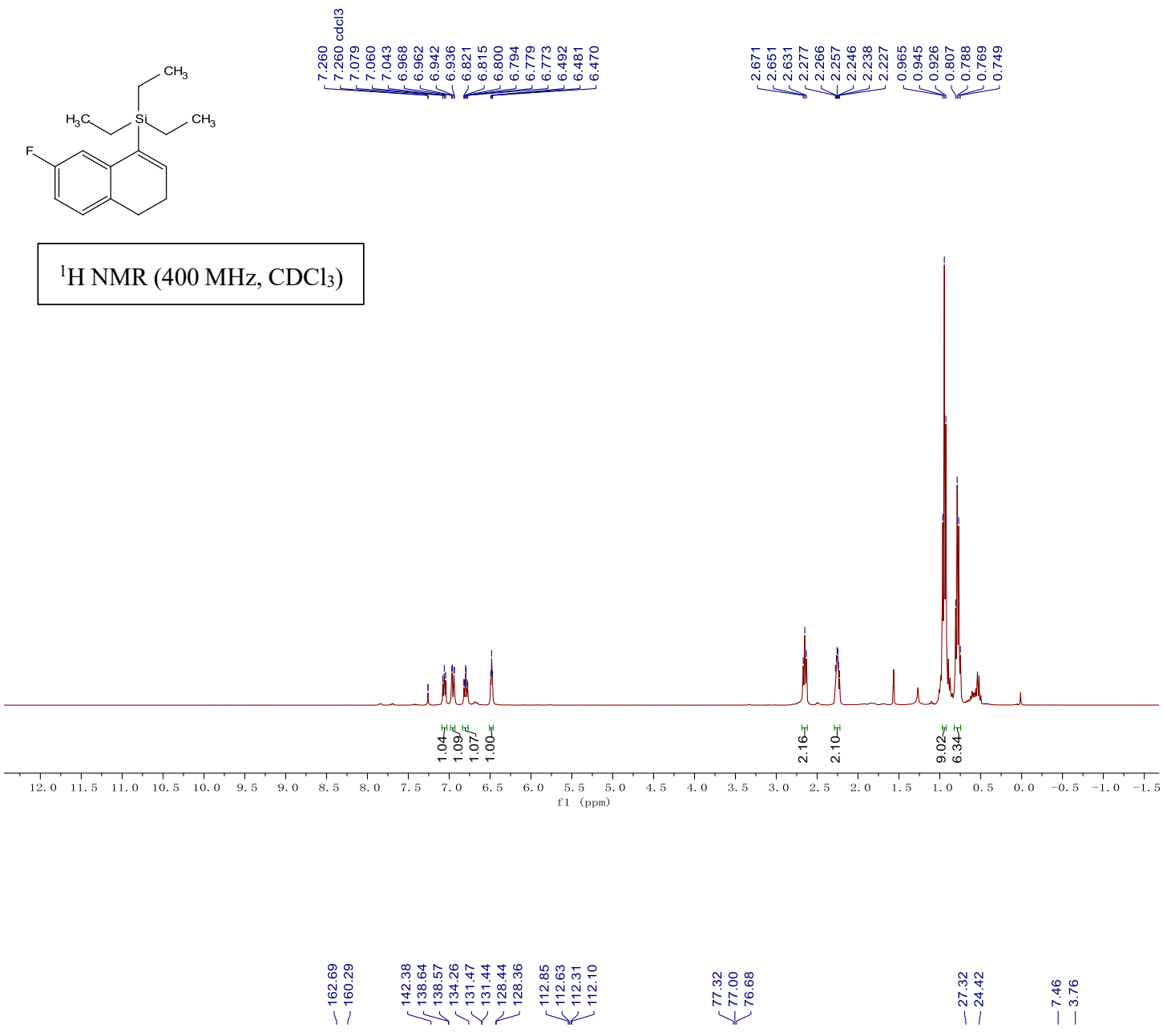

${ }^{13} \mathrm{C}$ NMR (100 MHz, $\left.\mathrm{CDCl}_{3}\right)$ 
Triethyl( $1 H$-inden-2-yl)silane (48)
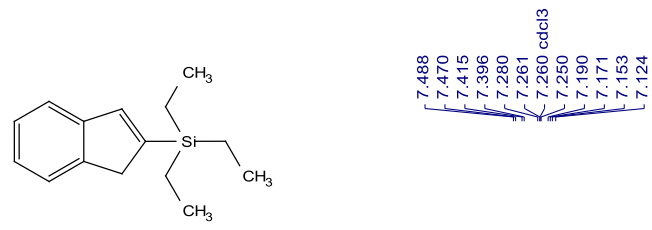

${ }^{1} \mathrm{H} \mathrm{NMR}\left(400 \mathrm{MHz}, \mathrm{CDCl}_{3}\right)$

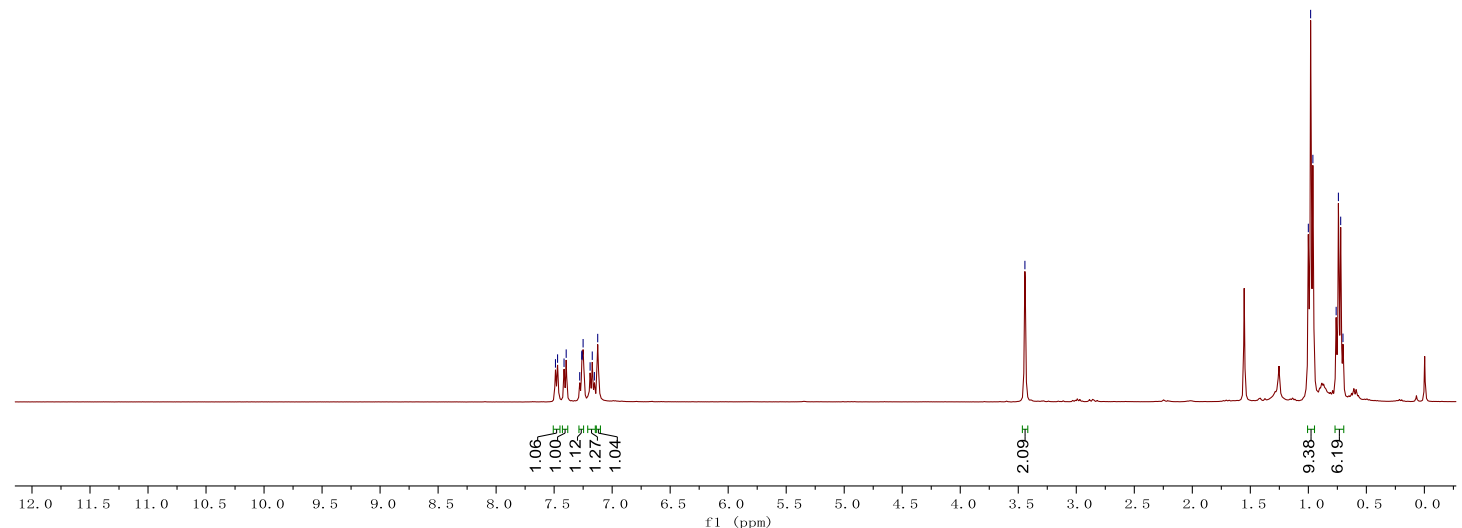

${ }^{13} \mathrm{C}$ NMR $\left(100 \mathrm{MHz}, \mathrm{CDCl}_{3}\right)$

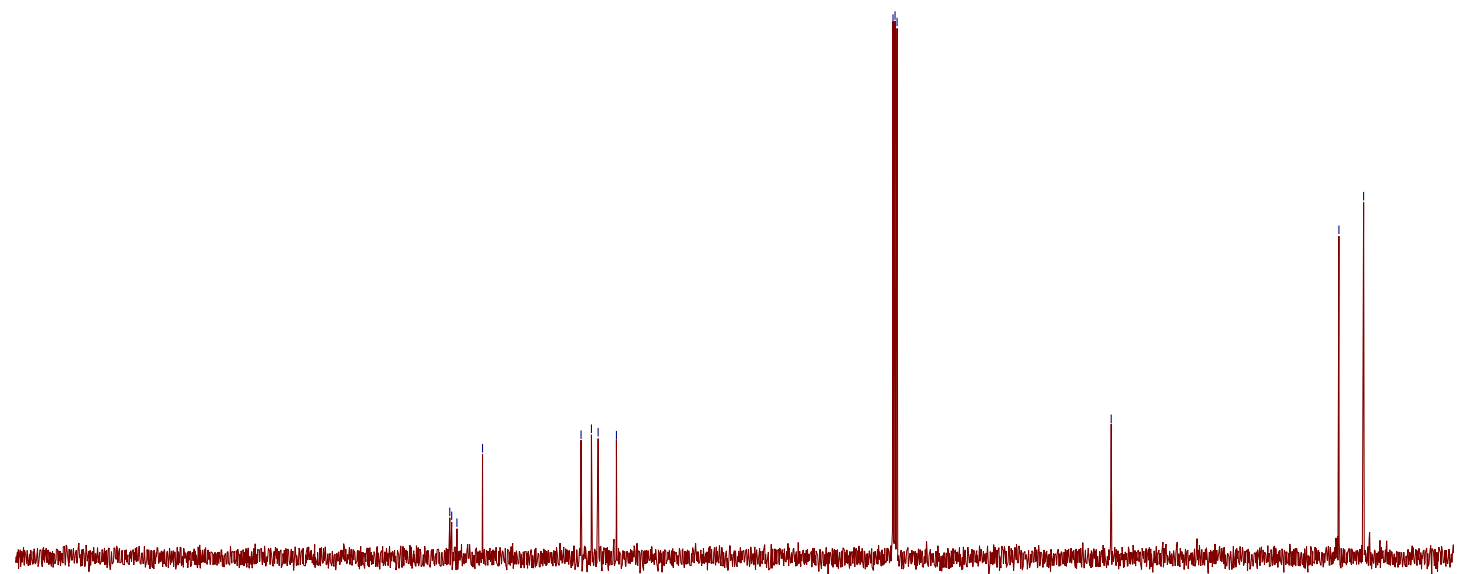

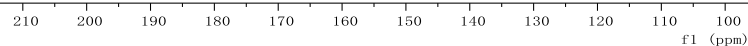


Triethyl(1,2,3,6-tetrahydro-[1,1'-biphenyl]-4-yl)silane (49)

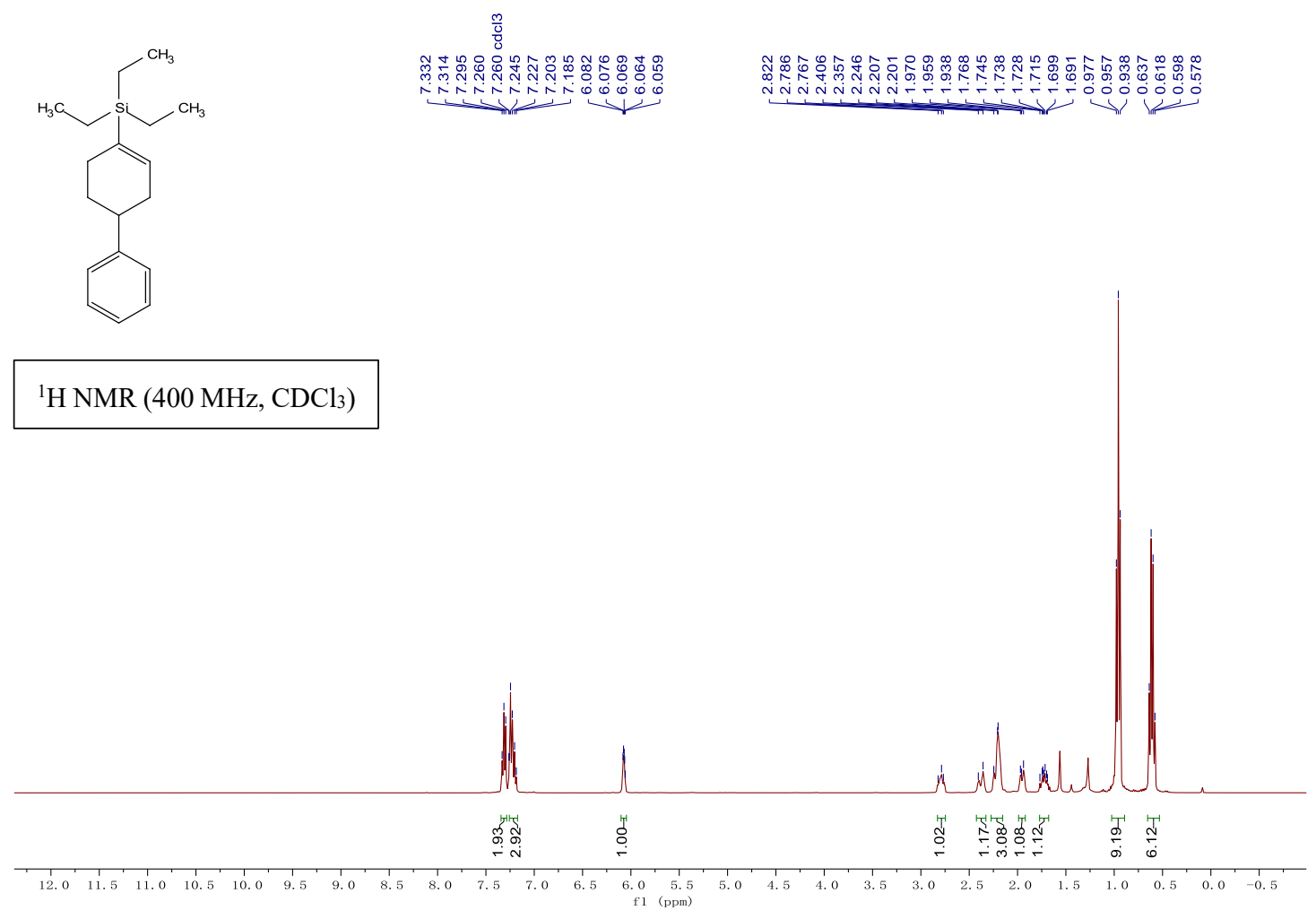

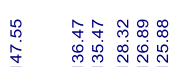

${ }^{13} \mathrm{C} \mathrm{NMR}\left(100 \mathrm{MHz}, \mathrm{CDCl}_{3}\right)$

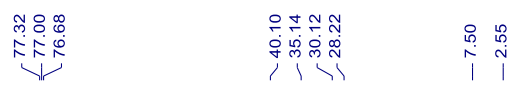

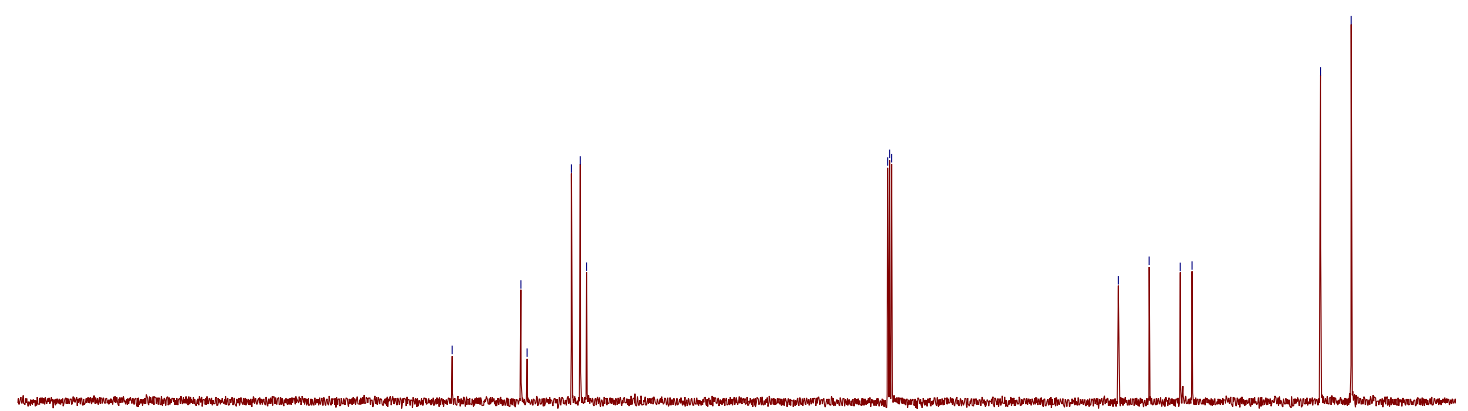

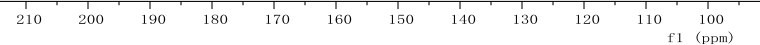


(4-(Benzyloxy)cyclohex-1-en-1-yl)triethylsilane (50)

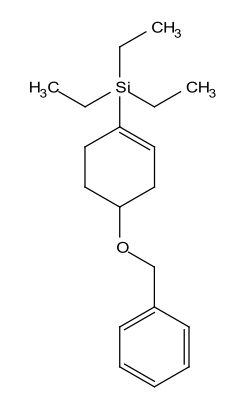

$\frac{m}{\frac{0}{8}}$

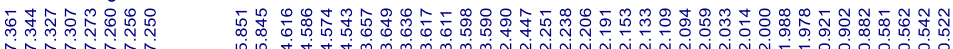

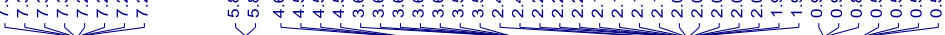

${ }^{1} \mathrm{H}$ NMR $\left(400 \mathrm{MHz}, \mathrm{CDCl}_{3}\right)$

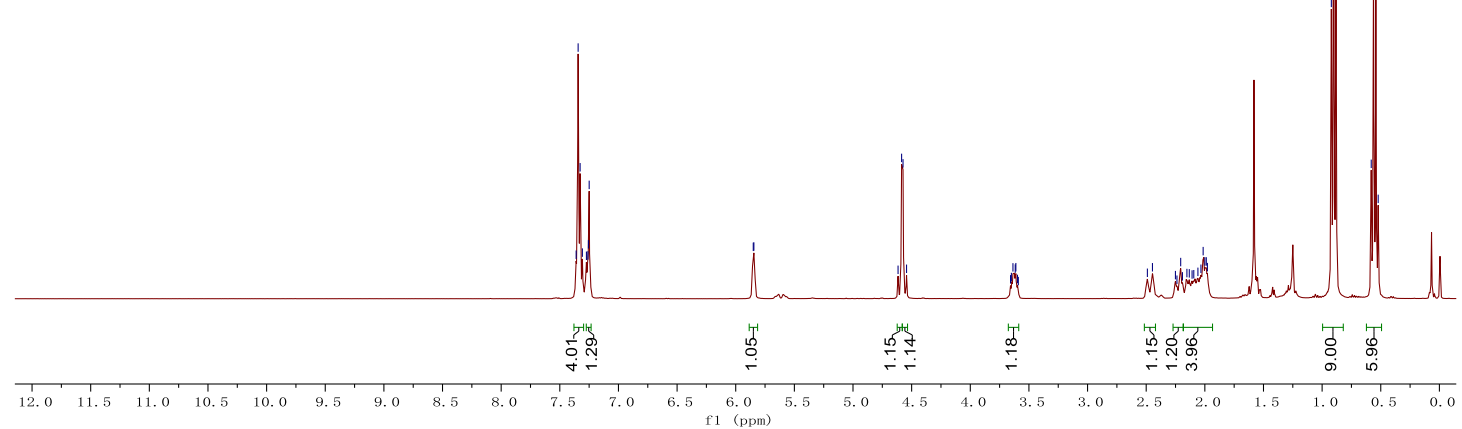

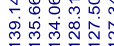

$\sqrt{5}$

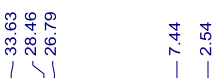

${ }^{13} \mathrm{C} \mathrm{NMR}\left(100 \mathrm{MHz}, \mathrm{CDCl}_{3}\right)$

परो

的去

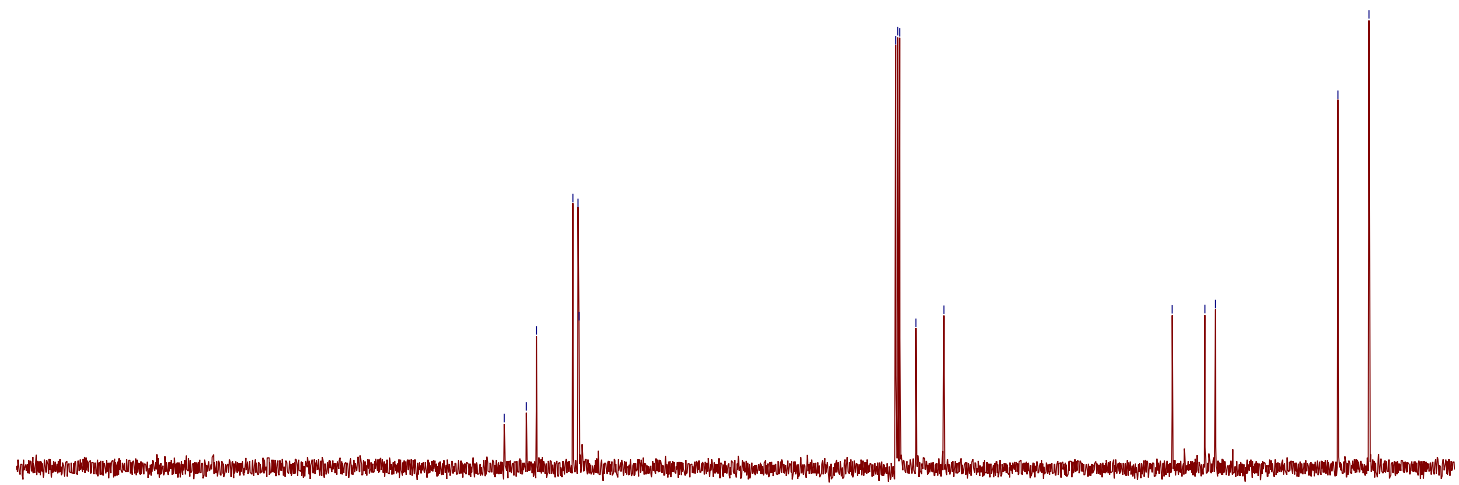

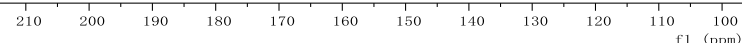


4'-(Triethylsilyl)-1',2',3',6'-tetrahydro-[1,1'-biphenyl]-4-yl (51)

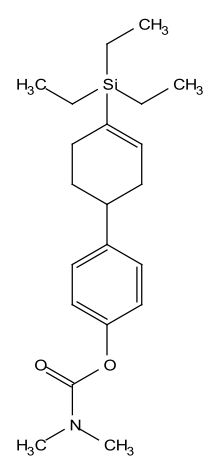

$\frac{m}{8}$

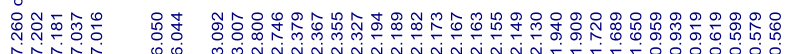

NiNi

${ }^{1} \mathrm{H}$ NMR (400 MHz, $\left.\mathrm{CDCl}_{3}\right)$

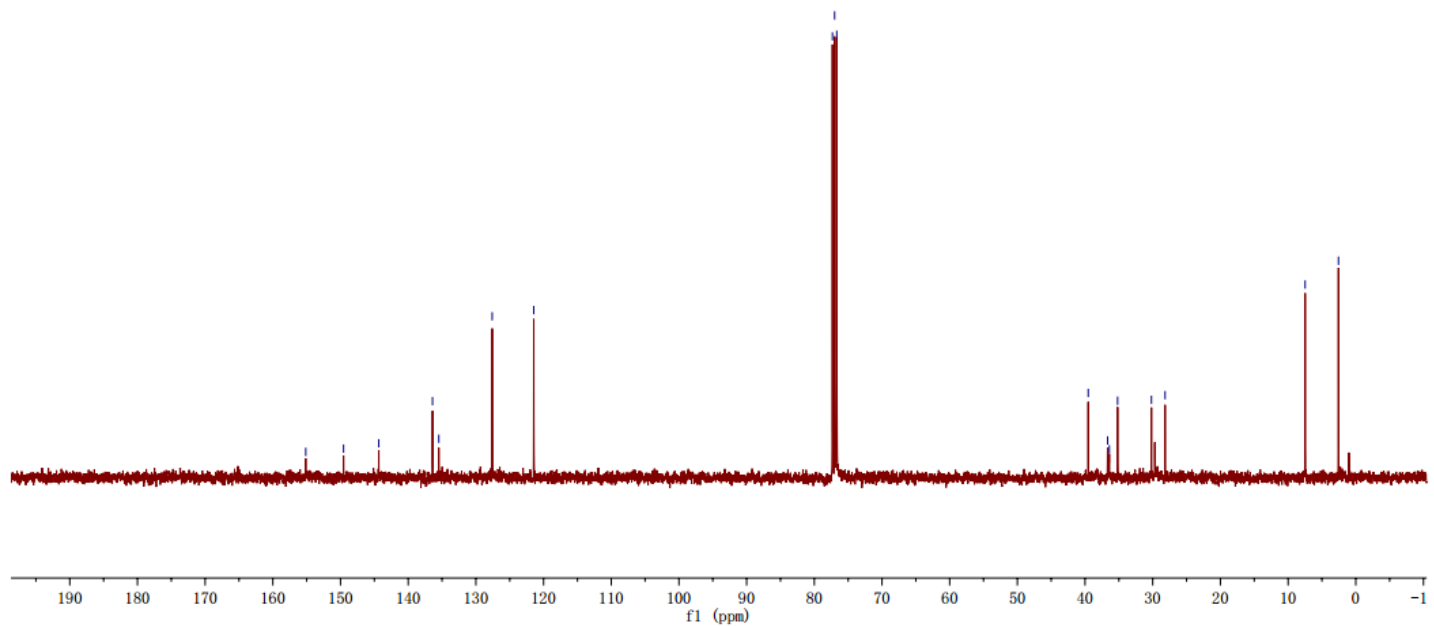


2-((4'-(Triethylsilyl)-1',2',3',6'-tetrahydro-[1,1'-biphenyl]-4-yl)oxy)pyridine (52)

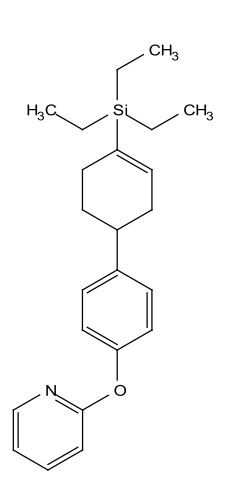

$\frac{m}{0}$

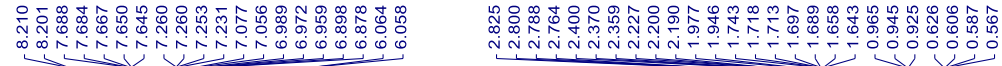

${ }^{1} \mathrm{H} \mathrm{NMR}\left(400 \mathrm{MHz}, \mathrm{CDCl}_{3}\right)$
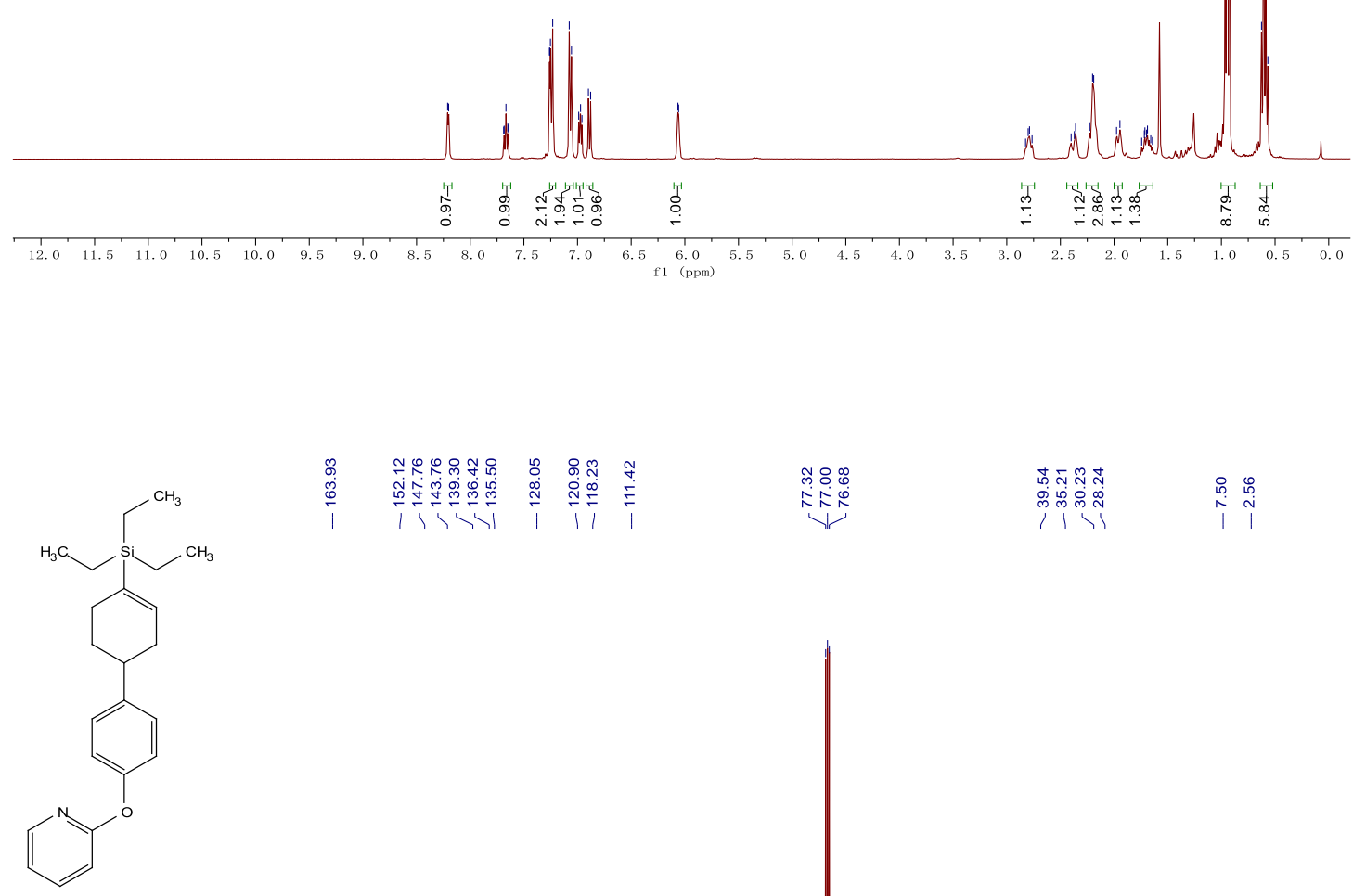

$\begin{array}{lllll}1 & 1 & 1 & -1 & 1\end{array}$

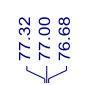

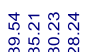

ili ili

${ }^{13} \mathrm{C} \mathrm{NMR}\left(100 \mathrm{MHz}, \mathrm{CDCl}_{3}\right)$

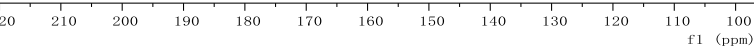


(3,3-Dimethylbut-1-en-2-yl)triethylsilane (53)

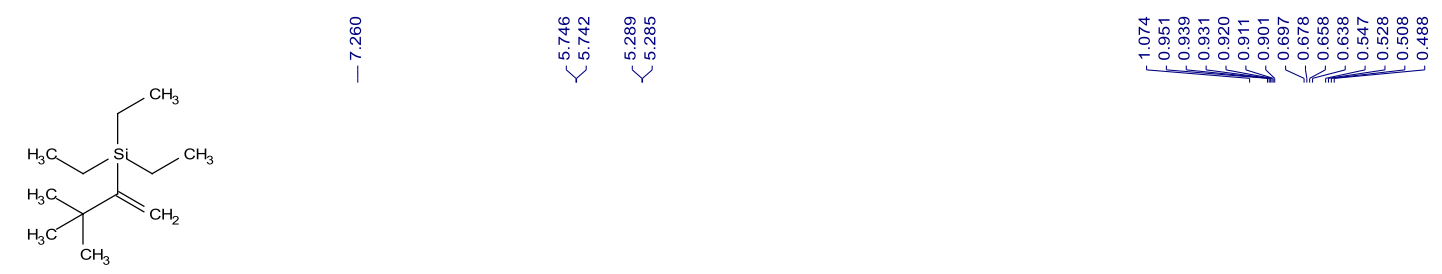

${ }^{1} \mathrm{H} \mathrm{NMR}\left(400 \mathrm{MHz}, \mathrm{CDCl}_{3}\right)$

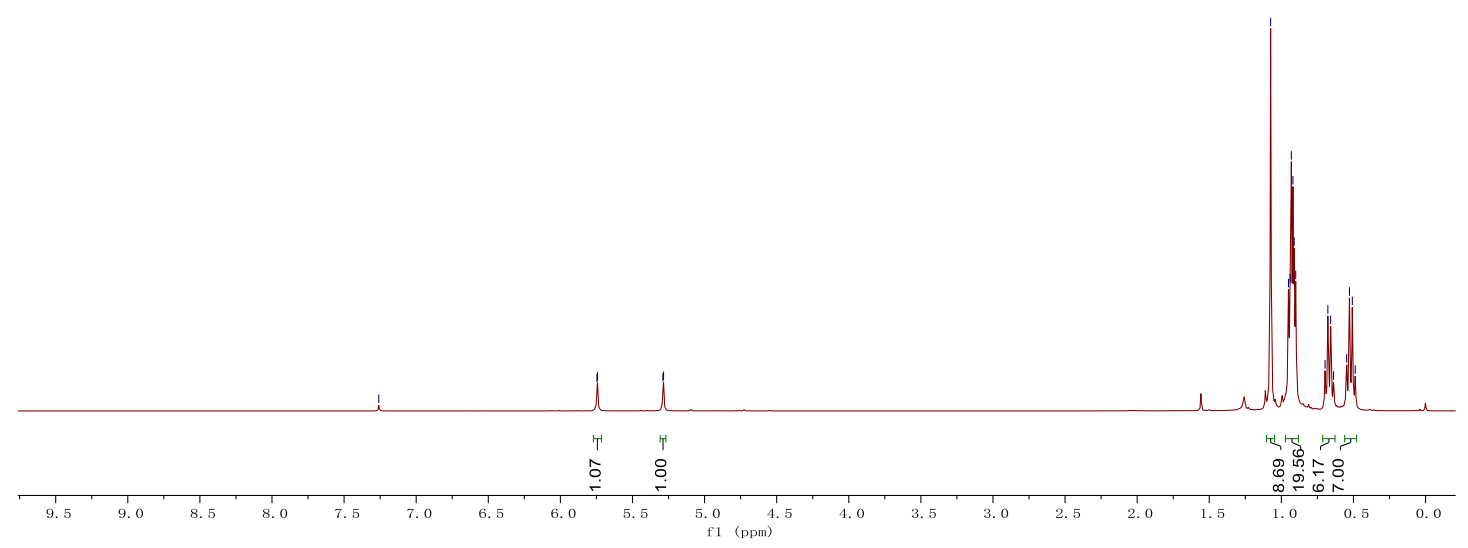


Triethyl(2-methyl-3,4-dihydronaphthalen-1-yl)silane (54)
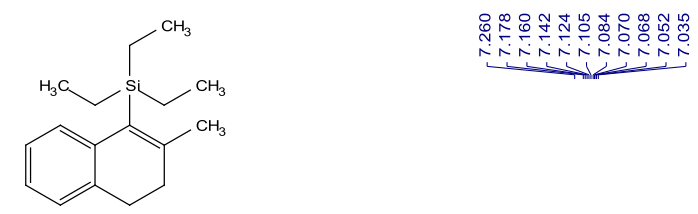

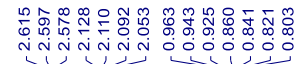

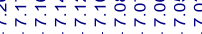

${ }^{1} \mathrm{H}$ NMR $\left(400 \mathrm{MHz}, \mathrm{CDCl}_{3}\right)$
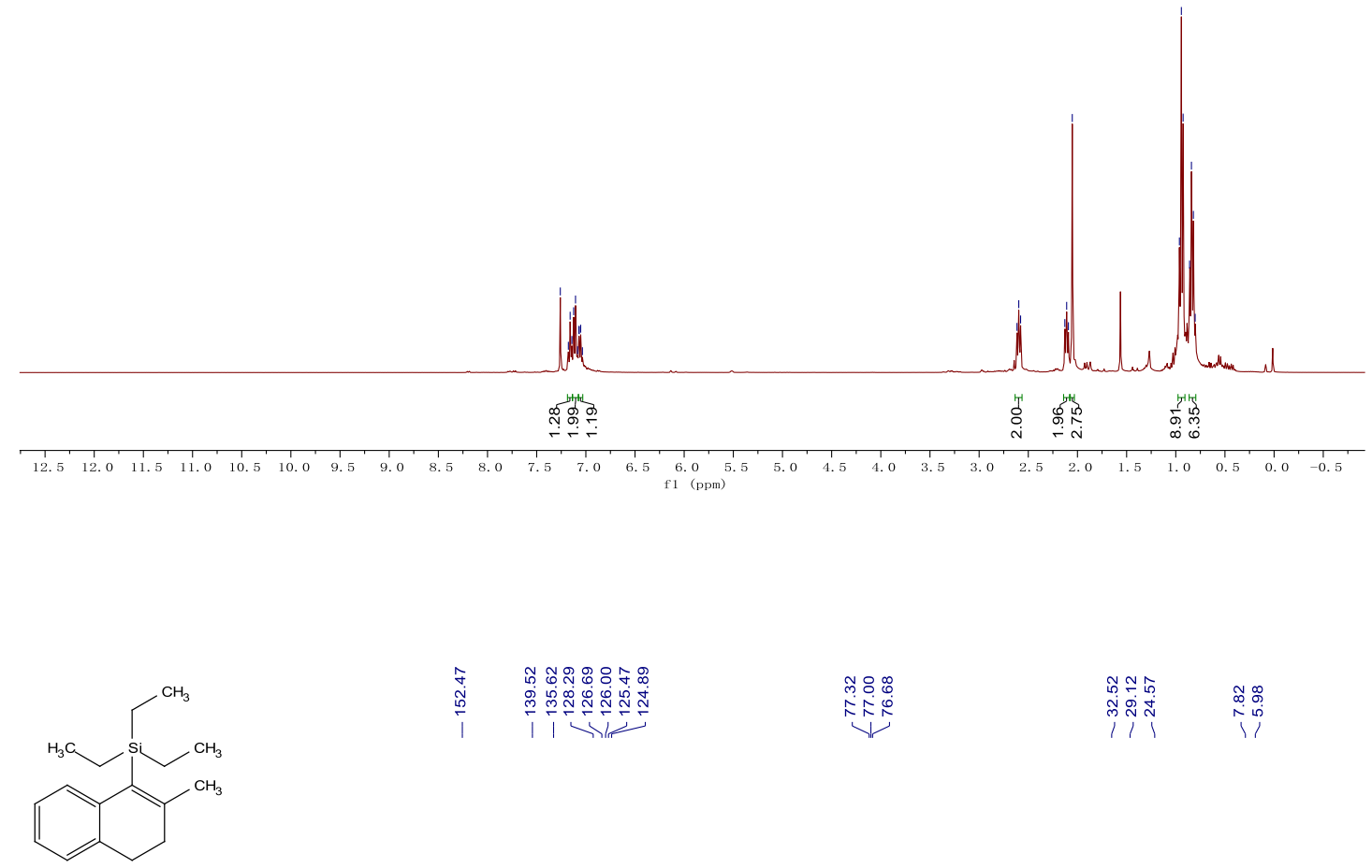

范

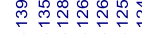

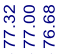

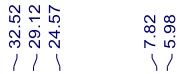

${ }^{13} \mathrm{C} \mathrm{NMR}\left(100 \mathrm{MHz}, \mathrm{CDCl}_{3}\right)$

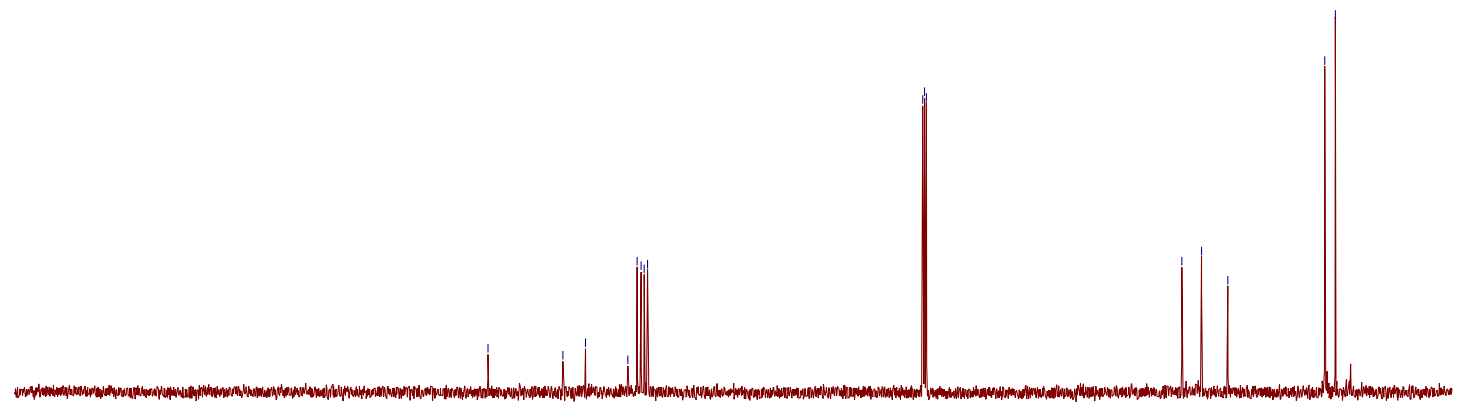


((8S,9S,14S)-6,7,8,9,11,12,13,14,15,16-decahydrospiro[cyclopenta[a]phenan-

threne-17,2'-[1,3]dioxolan]-3-yl)triethylsilane (55)

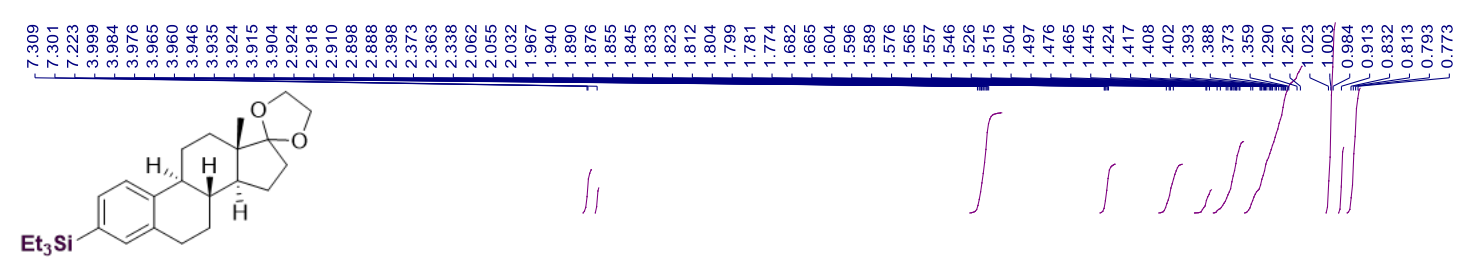

${ }^{1} \mathrm{H} \mathrm{NMR}\left(400 \mathrm{MHz}, \mathrm{CDCl}_{3}\right)$
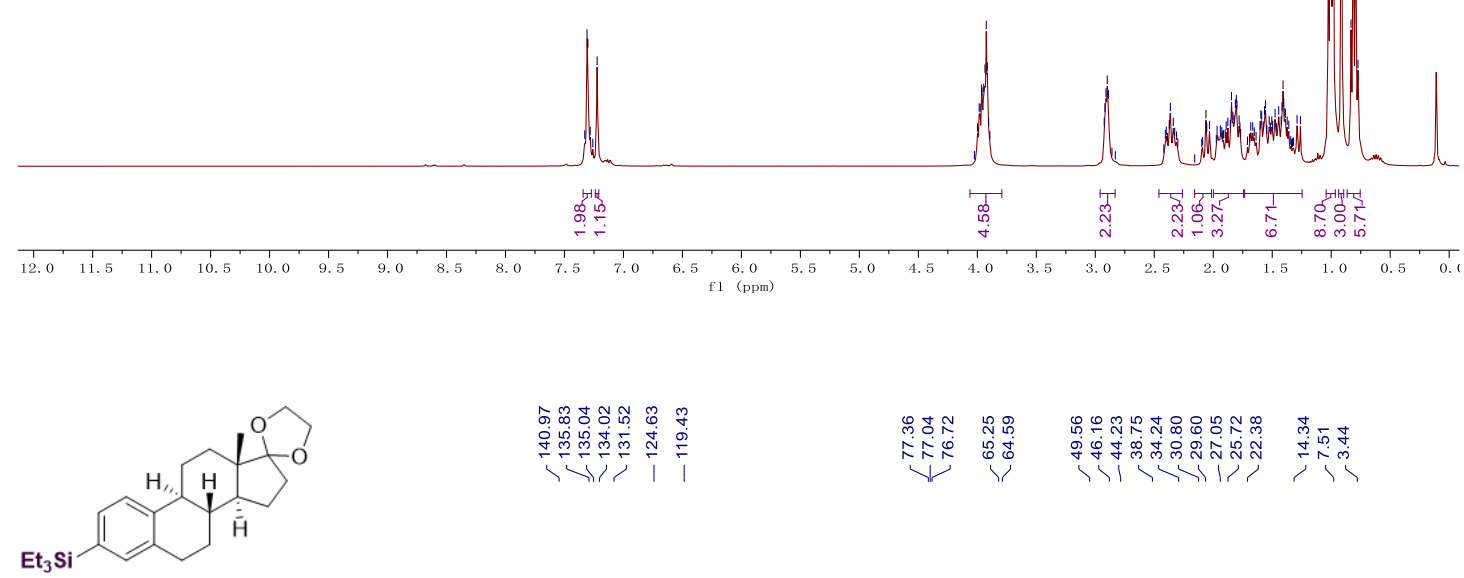

${ }^{13} \mathrm{C} \mathrm{NMR}\left(100 \mathrm{MHz}, \mathrm{CDCl}_{3}\right)$

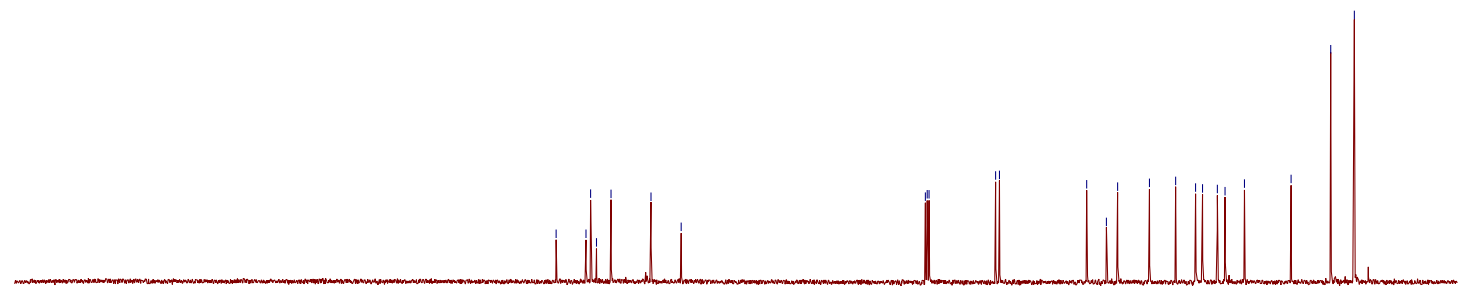

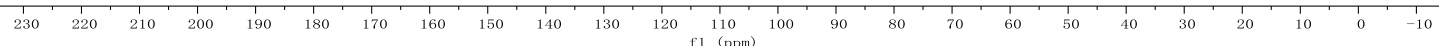


((S)-2,8-dimethyl-2-((4S,8S)-4,8,12-trimethyltridecyl)chroman-6-yl)triethyl-

silane (56)

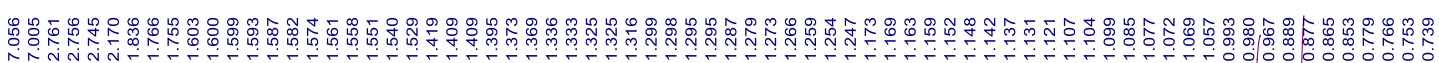

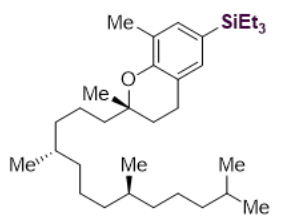

${ }^{1} \mathrm{H} \mathrm{NMR}\left(600 \mathrm{MHz}, \mathrm{CDCl}_{3}\right)$
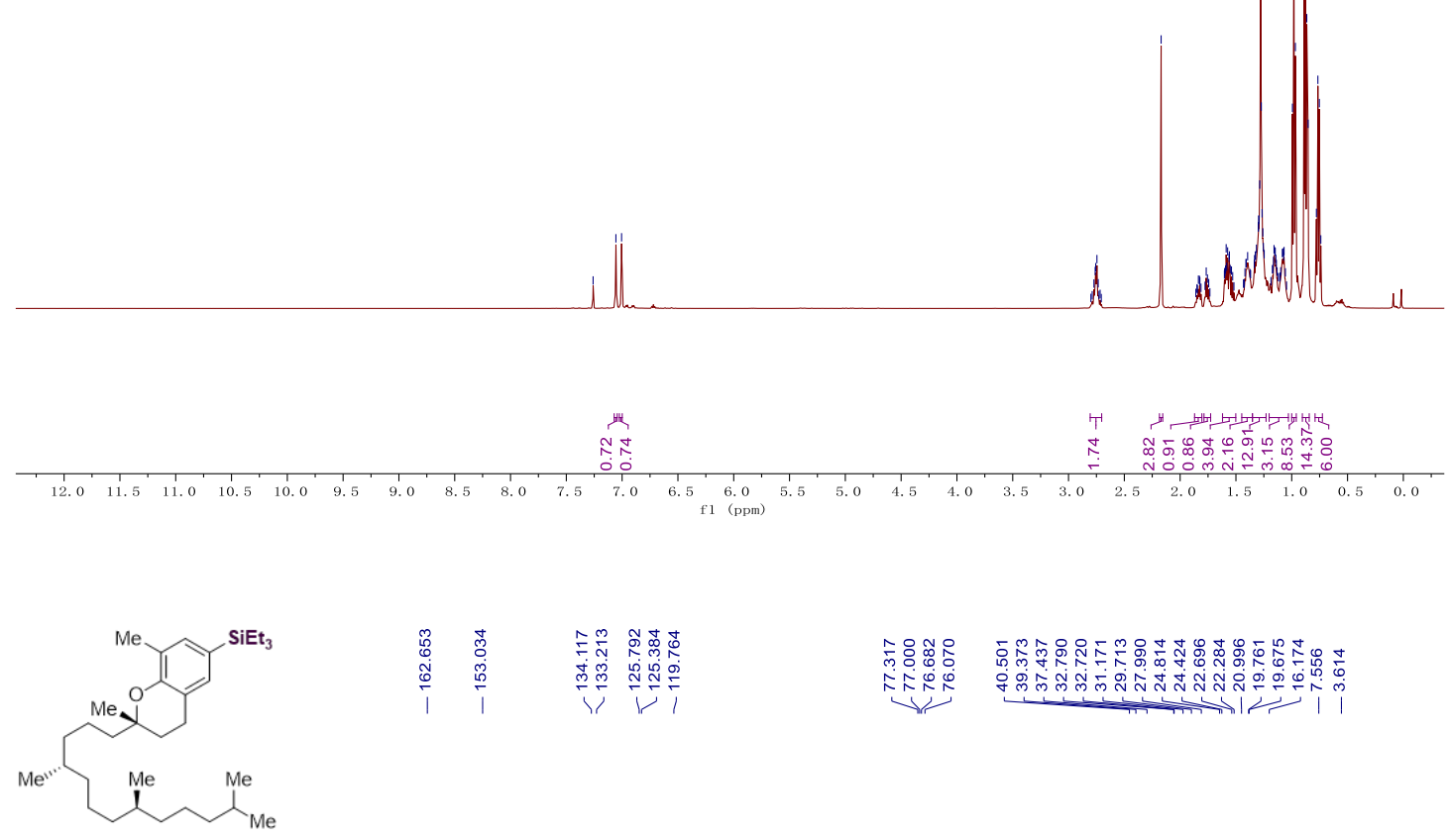

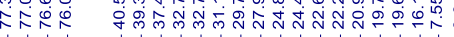

${ }^{13} \mathrm{C} \mathrm{NMR}\left(100 \mathrm{MHz}, \mathrm{CDCl}_{3}\right)$

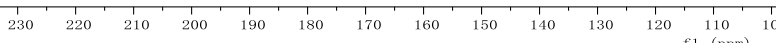


Triethyl(4-(3-methoxyphenyl)naphthalen-2-yl)silane (57)
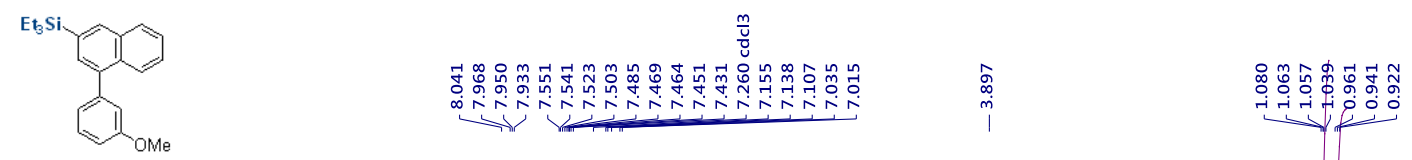

${ }^{1} \mathrm{H} \mathrm{NMR}\left(400 \mathrm{MHz}, \mathrm{CDCl}_{3}\right)$
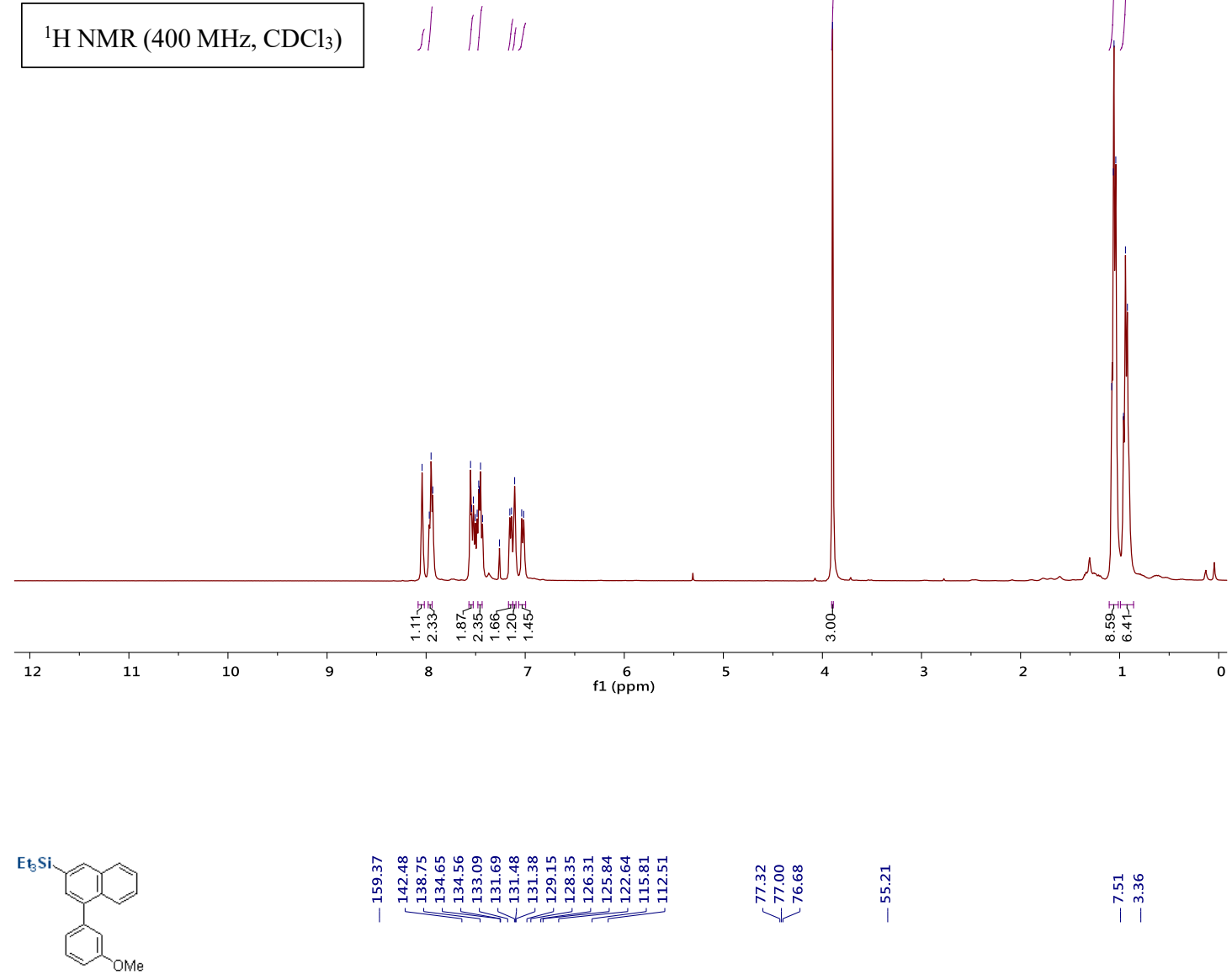

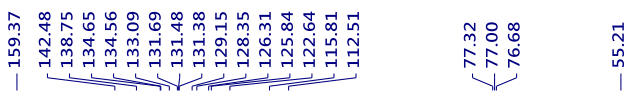

$\stackrel{\substack{n \\ i}}{\substack{m \\ m}}$

${ }^{13} \mathrm{C}$ NMR $\left(100 \mathrm{MHz}, \mathrm{CDCl}_{3}\right)$

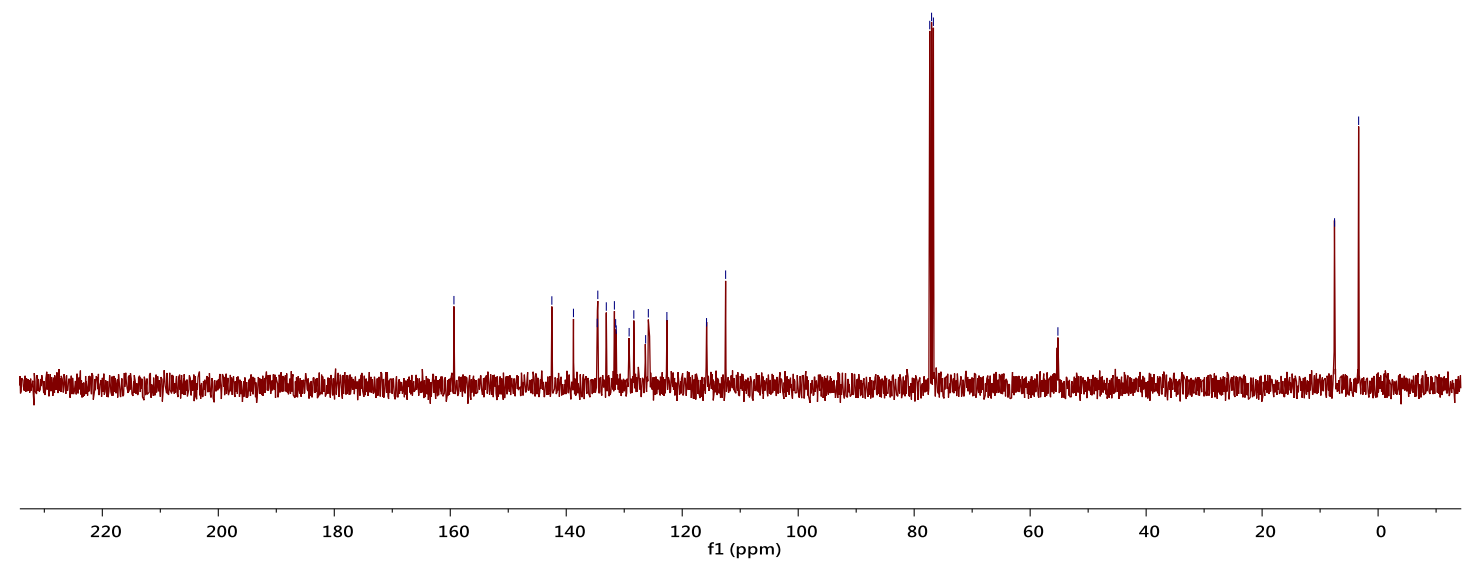


Triethyl(4-(4-(trifluoromethyl)phenyl)naphthalen-2-yl)silane (58)
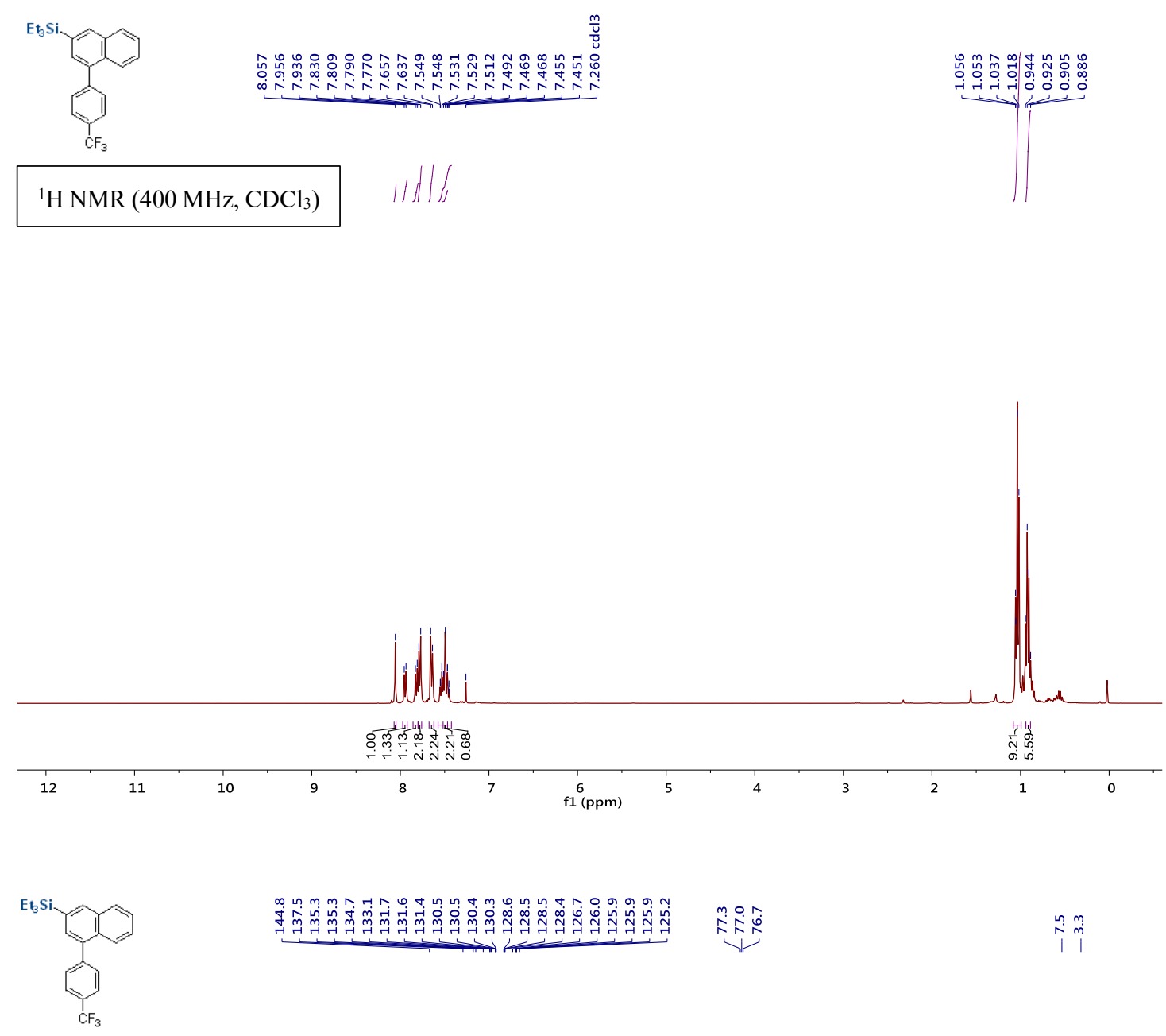

${ }^{13} \mathrm{C} \mathrm{NMR}\left(100 \mathrm{MHz}, \mathrm{CDCl}_{3}\right)$ 